Supporting Information

\title{
Rhodium(III)-Catalyzed Chemo-Divergent Couplings of Sulfoxonium Ylides with Oxa/Azabicyclic Olefins
}

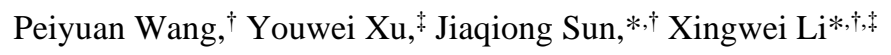 \\ $\dagger$ Key Laboratory of Applied Surface and Colloid Chemistry of MOE \& School of Chemistry and \\ Chemical Engineering, Shaanxi Normal University (SNNU), Xi'an 710062, China \\ $\ddagger$ Dalian Institute of Chemical Physics, Chinese Academy of Sciences, Dalian 116923, China \\ Email: sunjq@snnu.edu.cn; xwli@dicp.ac.cn; lixw@snnu.edu.cn
}

\section{Table of Contents}

I. General Information $\quad$ S1

II. Experiment Details and Analytical Data of 3, 5 and $6 \quad$ S2

$\begin{array}{lr}\text { III. Preparation of 3ba in Gram Scale } & \text { S17 }\end{array}$

$\begin{array}{ll}\text { IV. Derivatization of 3ba } & \text { S18 }\end{array}$

$\begin{array}{lr}\text { V. Mechanistic Studies } & \text { S19 }\end{array}$

VI. X-ray Crystal Diffraction Data for 3fa

$\begin{array}{ll}\text { VII. References } & \text { S23 }\end{array}$

VIII. ${ }^{1} \mathrm{H},{ }^{13} \mathrm{C}$ NMR Spectra of New Compounds. $\quad$ S24 


\section{General Information.}

All chemicals were obtained from commercial sources and were used as received unless otherwise noted. Sulfoxonium ylides, ${ }^{1}$ 7-Oxabenzonorbornadiene, ${ }^{2}$ and 7-Azabenzonorbornadiene ${ }^{3}$ were synthesized according to literature reports. The reactions were monitored with the aid of thin-layer chromatography (TLC) on $0.25 \mathrm{~mm}$ precoated silica gel plates. Visualization was carried out with UV light or aqueous potassium permanganate stain. ${ }^{1} \mathrm{H}$ NMR and ${ }^{13} \mathrm{C}$ NMR spectra were recorded at $25{ }^{\circ} \mathrm{C}$ on a JEOL $400 \mathrm{MHz}$ and $150 \mathrm{MHz}$ NMR spectrometers or Bruker $600 \mathrm{MHz}$ and $150 \mathrm{MHz}$ NMR spectrometers, respectively. Chemical shifts $(\delta)$ are given in ppm relative to TMS. The residual solvent signals were used as references for ${ }^{1} \mathrm{H}$ and ${ }^{13} \mathrm{C}$ NMR spectra and the chemical shifts were converted to the TMS scale (TMS: $\delta \mathrm{H}=0.00 \mathrm{ppm}, \mathrm{CDCl}_{3}: \delta \mathrm{H}=7.26 \mathrm{ppm}, \delta \mathrm{C}=77.16 \mathrm{ppm}, \mathrm{CD}_{3} \mathrm{CN}: \delta \mathrm{H}=1.94$ ppm, $\delta \mathrm{C}=1.32,118.26 \mathrm{ppm},\left(\mathrm{CD}_{3}\right)_{2} \mathrm{CO}: \delta \mathrm{H}=2.05 \mathrm{ppm}, \delta \mathrm{C}=29.84,206.26 \mathrm{ppm}$,). Coupling constants $(J)$ are given in Hertz $(\mathrm{Hz})$. Letters $\mathrm{m}, \mathrm{s}, \mathrm{d}, \mathrm{t}$ and q stand for multiplet, singlet, doublet, triplet, and quartet, respectively. High resolution mass spectra were recorded on Bruck Microtof. Column chromatography was performed on silica gel (300-400 mesh) using ethyl acetate (EA)/petroleum ether (PE). 
II. Experiment Details and Analytical Data of 3, 5 and 6.

1) Optimization Studies on the N-Annulation System.

Table S1. Optimization of Reaction Conditions of the N-Annulation ${ }^{a, b}$

\begin{tabular}{|c|c|c|c|}
\hline $1 a$ & $4 a$ & & $5 \mathbf{a a}$ \\
\hline Entry & solvent & $\mathbf{T}\left({ }^{\circ} \mathbf{C}\right)$ & yield $(\%)$ \\
\hline 1 & DCE & 80 & $52 \%$ \\
\hline 2 & $\mathrm{CHCl}_{3}$ & 80 & $43 \%$ \\
\hline 3 & $\mathrm{PhCl}$ & 80 & $60 \%$ \\
\hline 4 & DCM & 80 & $64 \%$ \\
\hline 5 & $\mathrm{DCM}$ & 120 & $50 \%$ \\
\hline 6 & DCM & 100 & $55 \%$ \\
\hline 7 & DCM & 60 & $68 \%$ \\
\hline $8^{f}$ & DCM & 60 & $70 \%$ \\
\hline $9^{c, f}$ & $\mathrm{DCM}$ & 60 & $72 \%$ \\
\hline $10^{d, f}$ & DCM & 60 & $82 \%$ \\
\hline $11^{e, f}$ & DCM & 60 & $63 \%$ \\
\hline
\end{tabular}

${ }^{a}$ Reaction conditions: 1a $(0.1 \mathrm{mmol}), \mathbf{4 a}\left(1.5\right.$ equiv), $\left[\mathrm{Cp} * \mathrm{Rh}(\mathrm{MeCN})_{3}\right]\left(\mathrm{SbF}_{6}\right)_{2}(8 \mathrm{~mol} \%)$, solvent $(2 \mathrm{~mL})$ under $\mathrm{N}_{2}$ for $20 \mathrm{~h} .{ }^{b}$ Isolated yields. ${ }^{c} \mathbf{1 a}(0.1 \mathrm{mmol}), \mathbf{4 a}\left(2.0\right.$ equiv). ${ }^{d} \mathbf{4 a}(0.1$ mmol), 1a (1.5 equiv). ${ }^{e} \mathbf{4 a}(0.1 \mathrm{mmol}), \mathbf{1 a}\left(2.0\right.$ equiv). ${ }^{f_{4}} \AA \mathrm{MS}(50 \mathrm{mg})$ was used. 
2) General procedure for preparation of 3 .

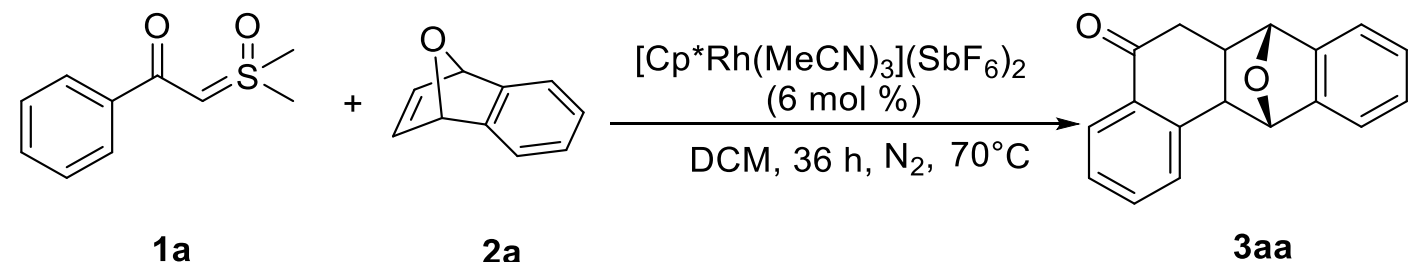

Representative Synthesis of Product 3: A pressure tube was charged with 1a (39.2 mg, $0.2 \mathrm{mmol})$, 2a (57.7 mg, $0.4 \mathrm{mmol}),\left[\mathrm{Cp} * \mathrm{Rh}(\mathrm{MeCN})_{3}\right]\left(\mathrm{SbF}_{6}\right)_{2}(10.0 \mathrm{mg}, 6 \mathrm{~mol} \%)$, and anhydrous DCM $(2.0 \mathrm{~mL})$. The reaction mixture was stirred at $70{ }^{\circ} \mathrm{C}$ for $36 \mathrm{~h}$ under $\mathrm{N}_{2}$. After the reaction was completed as indicated by TLC analysis, the solvent was removed under reduced pressure and the residue was purified by silica gel chromatography using petroleum ether/ethyl acetate 15:1 (v/v) to give the corresponding product 3aa $(46.0 \mathrm{mg}, 88 \%)$

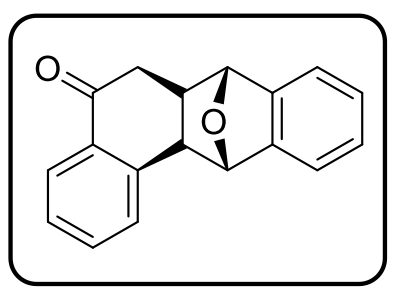

(rac)-6a,7,12,12a-tetrahydro-7,12-epoxytetraphen-5(6H)-one (3aa) Yellow solid (46.0 mg, 88\%), ${ }^{1} \mathrm{H}$ NMR $\left(400 \mathrm{MHz}, \mathrm{CD}_{3} \mathrm{CN}\right) \delta 7.72$ (dd, $\left.J_{1}=7.6 \mathrm{~Hz}, J_{2}=0.4 \mathrm{~Hz}, 1 \mathrm{H}\right), 7.66-7.58(\mathrm{~m}, 2 \mathrm{H}), 7.46-7.43(\mathrm{~m}, 1 \mathrm{H})$, $7.41-7.34(\mathrm{~m}, 2 \mathrm{H}), 7.27-7.22(\mathrm{~m}, 2 \mathrm{H}), 5.16(\mathrm{~s}, 1 \mathrm{H}), 4.92(\mathrm{~s}, 1 \mathrm{H}), 3.26$ $(\mathrm{d}, J=8.0 \mathrm{~Hz}, 1 \mathrm{H}), 2.82-2.78(\mathrm{~m}, 2 \mathrm{H}), 2.47\left(\mathrm{td}, J_{1}=7.2 \mathrm{~Hz}, J_{2}=3.6\right.$ $\mathrm{Hz}, 1 \mathrm{H}) .{ }^{13} \mathrm{C}$ NMR $\left(100 \mathrm{MHz}, \mathrm{CD}_{3} \mathrm{CN}\right) \delta 198.2,145.7,144.6,143.7$, 134.9, 133.4, 129.6, 127.1, 127.0, 126.7, 124.8, 119.7, 119.3, 87.8, 86.2, 43.5, 41.8, 37.8. HRMS (ESI-TOF) (m/z): Calcd for $\mathrm{C}_{18} \mathrm{H}_{14} \mathrm{NaO}_{2},\left([\mathrm{M}+\mathrm{Na}]^{+}\right), 285.0886$, found 285.0878 .

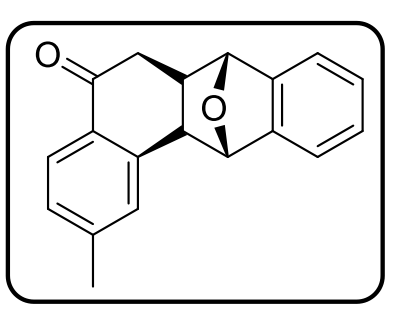

(rac)-2-methyl-6a,7,12,12a-tetrahydro-7,12-epoxytetraphen-5(6H)-o ne (3ba)

Yellow solid (48.9 mg, 89\%), ${ }^{1} \mathrm{H}$ NMR (400 MHz, $\left.\mathrm{CD}_{3} \mathrm{CN}\right) \delta 7.61(\mathrm{~d}, J$ $=7.6 \mathrm{~Hz}, 1 \mathrm{H}), 7.46-7.42(\mathrm{~m}, 2 \mathrm{H}), 7.38-7.33(\mathrm{~m}, 1 \mathrm{H}), 7.28-7.23(\mathrm{~m}$, $2 \mathrm{H}), 7.20(\mathrm{~d}, J=8.0 \mathrm{~Hz}, 1 \mathrm{H}), 5.15(\mathrm{~s}, 1 \mathrm{H}), 4.93(\mathrm{~s}, 1 \mathrm{H}), 3.21(\mathrm{~d}, J=7.6$ $\mathrm{Hz}, 1 \mathrm{H}), 2.80-2.77(\mathrm{~m}, 2 \mathrm{H}), 2.49-2.45(\mathrm{~m}, 1 \mathrm{H}), 2.43(\mathrm{~s}, 3 \mathrm{H}) .{ }^{13} \mathrm{C}$ NMR (100 MHz, $\left.\mathrm{CD}_{3} \mathrm{CN}\right) \delta 198.7,146.6,145.6,145.1,144.6,133.4,130.9,128.4,128.0,127.9,125.9$, 120.5, 120.2, 88.7, 87.2, 44.3, 42.6, 38.6, 21.8. HRMS (ESI-TOF) (m/z): Calcd for $\mathrm{C}_{19} \mathrm{H}_{16} \mathrm{NaO}_{2},([\mathrm{M}+$ $\mathrm{Na}]^{+}$), 299.1043, found 299.1038 .

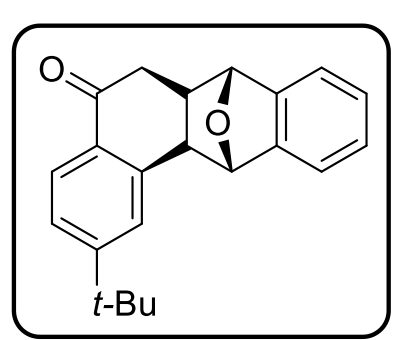

(rac)-2-(tert-butyl)-6a,7,12,12a-tetrahydro-7,12-epoxytetraphen-5(6 H)-one (3ca)

Yellow solid (39.2 mg, 62\%), ${ }^{1} \mathrm{H}$ NMR $\left(400 \mathrm{MHz}, \mathrm{CD}_{3} \mathrm{CN}\right) \delta 7.68-$ $7.63(\mathrm{~m}, 2 \mathrm{H}), 7.48(\mathrm{~d}, J=6.4 \mathrm{~Hz}, 1 \mathrm{H}), 7.44(\mathrm{~d}, J=8.0 \mathrm{~Hz}, 1 \mathrm{H}), 7.35(\mathrm{~d}$, $J=6.0 \mathrm{~Hz}, 1 \mathrm{H}), 7.28-7.21(\mathrm{~m}, 2 \mathrm{H}), 5.14(\mathrm{~s}, 1 \mathrm{H}), 4.95(\mathrm{~s}, 1 \mathrm{H}), 3.26(\mathrm{~d}$, $J=7.6 \mathrm{~Hz}, 1 \mathrm{H}), 2.82-2.76(\mathrm{~m}, 2 \mathrm{H}), 2.50-2.42(\mathrm{~m}, 1 \mathrm{H}), 1.38(\mathrm{~s}, 9 \mathrm{H})$. ${ }^{13} \mathrm{C}$ NMR $\left(100 \mathrm{MHz}, \mathrm{CD}_{3} \mathrm{CN}\right) \delta 198.7,158.0,146.7,145.6,144.3,133.3$, 127.9, 127.3, 125.6, 124.9, 124.8, 120.6, 120.1, 88.8, 88.6, 87.2, 87.1, 44.4, 42.6, 38.7, 35.8, 31.3. HRMS (ESI-TOF) (m/z): Calcd for $\mathrm{C}_{22} \mathrm{H}_{22} \mathrm{NaO}_{2}$, ([M + Na] $\left.]^{+}\right), 341.1512$, found 341.1505 . 


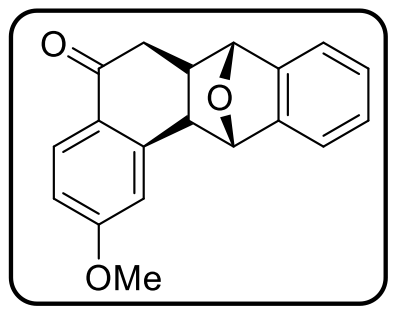

(rac)-2-methoxy-6a,7,12,12a-tetrahydro-7,12-epoxytetraphen-5(6H)one (3da)

Yellow solid (49.2 mg, 84\%), ${ }^{1} \mathrm{H}$ NMR (400 MHz, $\left.\mathrm{CD}_{3} \mathrm{CN}\right) \delta 7.69$ (d, $J$ $=8.8 \mathrm{~Hz}, 1 \mathrm{H}), 7.47-7.44(\mathrm{~m}, 1 \mathrm{H}), 7.36-7.33(\mathrm{~m}, 1 \mathrm{H}), 7.26-7.22(\mathrm{~m}$, $2 \mathrm{H}), 7.12(\mathrm{~d}, J=2.4 \mathrm{~Hz}, 1 \mathrm{H}), 6.90\left(\mathrm{dd}, J_{1}=8.8 \mathrm{~Hz}, J_{2}=2.4 \mathrm{~Hz}, 1 \mathrm{H}\right)$, $5.14(\mathrm{~s}, 1 \mathrm{H}), 5.00(\mathrm{~s}, 1 \mathrm{H}), 3.90(\mathrm{~s}, 3 \mathrm{H}), 3.20(\mathrm{~d}, J=8.0 \mathrm{~Hz}, 1 \mathrm{H}), 2.77$ $2.74(\mathrm{~m}, 2 \mathrm{H}), 2.47-2.41(\mathrm{~m}, 1 \mathrm{H}) .{ }^{13} \mathrm{C} \mathrm{NMR}\left(100 \mathrm{MHz}, \mathrm{CD}_{3} \mathrm{CN}\right) \delta 197.5,164.6,146.9,146.6,145.6$, $129.0,128.0,127.9,127.8,120.5,120.1,114.2,114.1,88.6,87.2,56.3,44.5,42.3$, 38.6. HRMS (ESI-TOF) $(\mathrm{m} / \mathrm{z})$ : Calcd for $\mathrm{C}_{19} \mathrm{H}_{16} \mathrm{NaO}_{3},\left([\mathrm{M}+\mathrm{Na}]^{+}\right), 315.0992$, found 315.0989 .

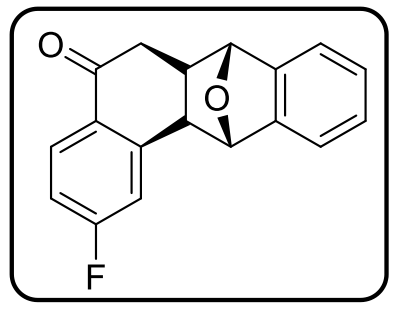

(rac)-2-fluoro-6a,7,12,12a-tetrahydro-7,12-epoxytetraphen-5(6H)-on e (3ea)

Yellow solid (32.3 mg, 58\%), ${ }^{1} \mathrm{H}$ NMR (400 MHz, $\left.\mathrm{CDCl}_{3}\right) \delta 7.89\left(\mathrm{dd}, J_{1}\right.$ $\left.=8.4 \mathrm{~Hz}, J_{2}=6.0 \mathrm{~Hz}, 1 \mathrm{H}\right), 7.40-7.33(\mathrm{~m}, 2 \mathrm{H}), 7.26-7.23(\mathrm{~m}, 2 \mathrm{H})$, $7.16\left(\mathrm{dd}, J_{1}=9.2 \mathrm{~Hz}, J_{2}=2.4 \mathrm{~Hz}, 1 \mathrm{H}\right), 7.09-7.03(\mathrm{~m}, 1 \mathrm{H}), 5.19(\mathrm{~s}, 1 \mathrm{H})$, $5.03(\mathrm{~s}, 1 \mathrm{H}), 3.23(\mathrm{~d}, J=8.0 \mathrm{~Hz}, 1 \mathrm{H}), 2.93\left(\mathrm{dd}, J_{1}=15.6 \mathrm{~Hz}, J_{2}=2.8\right.$ $\mathrm{Hz}, 1 \mathrm{H}), 2.82\left(\mathrm{dd}, J_{1}=15.2 \mathrm{~Hz}, J_{2}=8.0 \mathrm{~Hz}, 1 \mathrm{H}\right), 2.55\left(\mathrm{td}, J_{1}=8.0 \mathrm{~Hz}, J_{2}=2.8 \mathrm{~Hz}, 1 \mathrm{H}\right) .{ }^{13} \mathrm{C} \mathrm{NMR}$ $\left(100 \mathrm{MHz}, \mathrm{CDCl}_{3}\right) \delta 196.6,166.0(J=252.9 \mathrm{~Hz}), 145.9,145.0,144.2,131.32,131.30,128.9(J=9.4$ $\mathrm{Hz}), 127.5(J=9.2 \mathrm{~Hz}), 119.5(J=2.1 \mathrm{~Hz}), 115.4(J=21.3 \mathrm{~Hz}), 114.7(J=21.8 \mathrm{~Hz}), 87.8,86.5,44.2$, 42.0, 37.9. HRMS (ESI-TOF) (m/z): Calcd for $\mathrm{C}_{18} \mathrm{H}_{13} \mathrm{FNaO}_{2},\left([\mathrm{M}+\mathrm{Na}]^{+}\right), 303.0792$, found 303.0788.

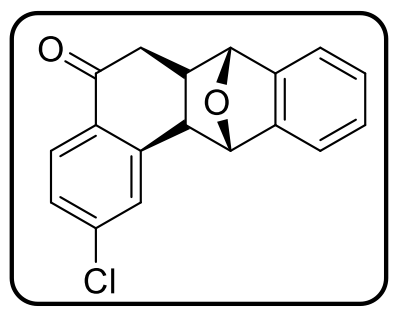

(rac)-2-chloro-6a,7,12,12a-tetrahydro-7,12-epoxytetraphen-5(6H)-on e (3fa)

Yellow solid (35.5 mg, 60\%), ${ }^{1} \mathrm{H}$ NMR $\left(400 \mathrm{MHz}, \mathrm{CDCl}_{3}\right) \delta 7.80(\mathrm{~d}, J=$ $8.4 \mathrm{~Hz}, 1 \mathrm{H}), 7.48$ (d, $J=2 \mathrm{~Hz}, 1 \mathrm{H}), 7.40-7.32$ (m, 3H), $7.26-7.23$ (m, $2 \mathrm{H}), 5.19(\mathrm{~s}, 1 \mathrm{H}), 5.02(\mathrm{~s}, 1 \mathrm{H}), 3.22(\mathrm{~d}, J=8.0 \mathrm{~Hz}, 1 \mathrm{H}), 2.94\left(\mathrm{dd}, J_{1}=\right.$ $\left.15.2 \mathrm{~Hz}, J_{2}=2.8 \mathrm{~Hz}, 1 \mathrm{H}\right), 2.81\left(\mathrm{dd}, J_{1}=15.6 \mathrm{~Hz}, J_{2}=8.4 \mathrm{~Hz}, 1 \mathrm{H}\right), 2.55$ $\left(\operatorname{td}, J_{1}=8.4 \mathrm{~Hz}, J_{2}=2.8 \mathrm{~Hz}, 1 \mathrm{H}\right),{ }^{13} \mathrm{C} \mathrm{NMR}\left(100 \mathrm{MHz}, \mathrm{CDCl}_{3}\right) \delta 197.0,145.0,144.8,144.1,139.7$, 133.1, 129.0, 127.58, 127.55, 127.5, 119.5, 90.9, 86.5, 43.9, 42.1, 38.0. HRMS (ESI-TOF) (m/z): Calcd for $\mathrm{C}_{18} \mathrm{H}_{13} \mathrm{ClNaO}_{2},([\mathrm{M}+\mathrm{Na}]+), 319.0496$, found 319.0498 .

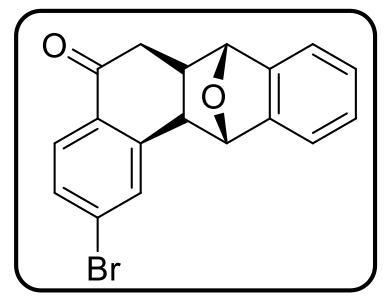

(rac)-2-bromo-6a,7,12,12a-tetrahydro-7,12-epoxytetraphen-5(6H)-on e (3ga)

Yellow solid (44.2 mg, 65\%), ${ }^{1} \mathrm{H}$ NMR (400 MHz, $\left.\mathrm{CD}_{3} \mathrm{CN}\right) \delta 7.86(\mathrm{~d}, J=$ $2.0 \mathrm{~Hz}, 1 \mathrm{H}), 7.61(\mathrm{~d}, J=8.4 \mathrm{~Hz}, 1 \mathrm{H}), 7.54\left(\mathrm{dd}, J_{1}=8.4 \mathrm{~Hz}, J_{2}=2.0 \mathrm{~Hz}\right.$, $1 \mathrm{H}), 7.47-7.44(\mathrm{~m}, 1 \mathrm{H}), 7.38-7.34(\mathrm{~m}, 1 \mathrm{H}), 7.28-7.22(\mathrm{~m}, 2 \mathrm{H}), 5.16$ $(\mathrm{s}, 1 \mathrm{H}), 4.97(\mathrm{~s}, 1 \mathrm{H}), 3.26(\mathrm{~d}, J=8.0 \mathrm{~Hz}, 1 \mathrm{H}), 2.83-2.78(\mathrm{~m}, 2 \mathrm{H}), 2.53$ - 2.44 (m, 1H). ${ }^{13} \mathrm{C}$ NMR (100 MHz, $\left.\mathrm{CD}_{3} \mathrm{CN}\right) \delta 198.3,146.7,146.5,145.3,134.7,133.3,130.8,128.2$, 128.1, 128.0, 127.7, 120.7, 120.2, 88.4, 87.1, 44.2, 42.5, 38.8. HRMS (ESI-TOF) (m/z): Calcd for $\mathrm{C}_{18} \mathrm{H}_{13} \mathrm{BrNaO}_{2},\left([\mathrm{M}+\mathrm{Na}]^{+}\right), 362.9991$, found 362.9986 . 


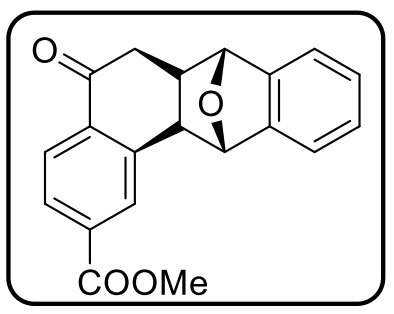

(rac)-5-oxo-5,6,6a,7,12,12a-hexahydro-7,12-epoxytetraphen-2-yl acetate (3ha)

Yellow solid (45.5 mg, 71\%), ${ }_{1}^{1} \mathrm{H}$ NMR (400 MHz, $\left.\mathrm{CD}_{3} \mathrm{CN}\right) \delta 8.28-8.26$ $(\mathrm{m}, 1 \mathrm{H}), 7.96\left(\mathrm{dd}, J_{1}=8.0 \mathrm{~Hz}, J_{2}=1.6 \mathrm{~Hz}, 1 \mathrm{H}\right), 7.78(\mathrm{~d}, J=8.4 \mathrm{~Hz}, 1 \mathrm{H})$, $7.50-7.46(\mathrm{~m}, 1 \mathrm{H}), 7.39-7.35(\mathrm{~m}, 1 \mathrm{H}), 7.28-7.23(\mathrm{~m}, 2 \mathrm{H}), 5.18(\mathrm{~s}$, $1 \mathrm{H}), 4.94(\mathrm{~s}, 1 \mathrm{H}), 3.93$ (s, 3H), 3.38 (d, J=7.6 Hz, 1H), $2.88-2.80$ (m, $2 \mathrm{H}), 2.52\left(\mathrm{td}, J_{1}=7.6 \mathrm{~Hz}, J_{2}=2.8 \mathrm{~Hz}, 1 \mathrm{H}\right) .{ }^{13} \mathrm{C} \mathrm{NMR}\left(100 \mathrm{MHz}, \mathrm{CD}_{3} \mathrm{CN}\right) \delta 198.8,167.0,146.5$, 145.2 , 144.8, 139.0, 135.2, 131.7, 128.11, 128.05, 127.9, 126.0, 120.8, 120.2, 88.4, 87.0, 53.0, 44.3, 42.8, 38.9. HRMS (ESI-TOF) (m/z): Calcd for $\mathrm{C}_{20} \mathrm{H}_{16} \mathrm{NaO}_{4},\left([\mathrm{M}+\mathrm{Na}]^{+}\right), 343.0941$, found 343.0935.

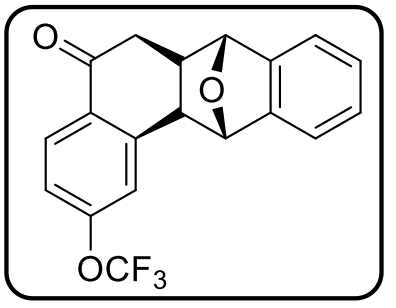

(rac)-2-(trifluoromethoxy)-6a,7,12,12a-tetrahydro-7,12-epoxytetrap hen-5(6H)-one (3ia)

Yellow solid (43.7 mg, 63\%), ${ }^{1} \mathrm{H}$ NMR (400 MHz, $\left.\mathrm{CD}_{3} \mathrm{CN}\right) \delta 7.81(\mathrm{~d}, J$ $=8.4 \mathrm{~Hz}, 1 \mathrm{H}), 7.58-7.56(\mathrm{~m}, 1 \mathrm{H}), 7.48-7.45(\mathrm{~m}, 1 \mathrm{H}), 7.38-7.33(\mathrm{~m}$, $1 \mathrm{H}), 7.31-7.22(\mathrm{~m}, 3 \mathrm{H}), 5.17(\mathrm{~s}, 1 \mathrm{H}), 4.97(\mathrm{~s}, 1 \mathrm{H}), 3.31(\mathrm{~d}, J=8.0 \mathrm{~Hz}$, $1 \mathrm{H}), 2.87-2.77(\mathrm{~m}, 2 \mathrm{H}), 2.50\left(\mathrm{td}, J_{1}=7.6 \mathrm{~Hz}, J_{2}=3.6 \mathrm{~Hz}, 1 \mathrm{H}\right) .{ }^{13} \mathrm{C}$ NMR $\left(150 \mathrm{MHz}, \mathrm{CDCl}_{3}\right) \delta$ 196.5, 152.9, 145.4, 145.0, 144.1, 133.2, 128.2, 127.5, 127.4, 120.6, 120.5 $(J=256.5 \mathrm{~Hz}), 119.6,119.5,119.1,87.7,86.4,44.2,42.0,38.0$. HRMS (ESI-TOF) (m/z): Calcd for $\mathrm{C}_{19} \mathrm{H}_{13} \mathrm{~F}_{3} \mathrm{NaO}_{3},\left([\mathrm{M}+\mathrm{Na}]^{+}\right), 369.0709$, found 369.0709.

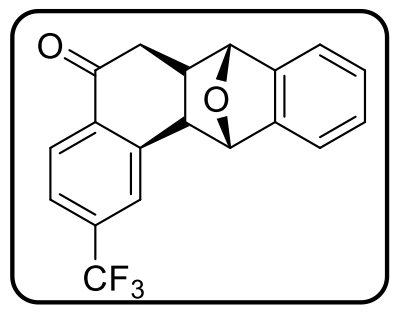

(rac)-2-(trifluoromethyl)-6a,7,12,12a-tetrahydro-7,12-epoxytetraphe n-5(6H)-one (3ja)

Yellow solid (45.4 mg, 69\%), ${ }^{1} \mathrm{H}$ NMR (400 MHz, $\left.\mathrm{CD}_{3} \mathrm{CN}\right) \delta 8.01(\mathrm{~d}, J$ $=0.8 \mathrm{~Hz}, 1 \mathrm{H}), 7.86(\mathrm{~d}, J=8.0 \mathrm{~Hz}, 1 \mathrm{H}), 7.68\left(\mathrm{dd}, J_{1}=8.0 \mathrm{~Hz}, J_{2}=1.2\right.$ $\mathrm{Hz}, 1 \mathrm{H}) 7.50-7.45(\mathrm{~m}, 1 \mathrm{H}), 7.39-7.34(\mathrm{~m}, 1 \mathrm{H}), 7.30-7.22(\mathrm{~m}, 2 \mathrm{H})$, $5.19(\mathrm{~s}, 1 \mathrm{H}), 4.97(\mathrm{~s}, 1 \mathrm{H}), 3.37(\mathrm{~d}, J=8.0 \mathrm{~Hz}, 1 \mathrm{H}), 2.92-2.79(\mathrm{~m}, 2 \mathrm{H})$, $2.53\left(\mathrm{td}, J_{1}=7.6 \mathrm{~Hz}, J_{2}=2.8 \mathrm{~Hz}, 1 \mathrm{H}\right) .{ }^{13} \mathrm{C} \mathrm{NMR}\left(150 \mathrm{MHz}, \mathrm{CDCl}_{3}\right) \delta 197.0,145.0,144.0,143.7$, 137.4, $135.0(\mathrm{~J}=32.4 \mathrm{~Hz}), 127.64,127.56,126.6,126.2(\mathrm{~J}=3.6 \mathrm{~Hz}), 123.82(\mathrm{~J}=3.6 \mathrm{~Hz}), 123.78(\mathrm{~J}=$ 271.2 Hz), 119.7, 119.5, 87.7, 86.4, 44.2, 42.3, 38.1. HRMS (ESI-TOF) (m/z): Calcd for $\mathrm{C}_{19} \mathrm{H}_{13} \mathrm{~F}_{3} \mathrm{NaO}_{2}$, $\left([\mathrm{M}+\mathrm{Na}]^{+}\right), 353.0760$, found 353.0754 .

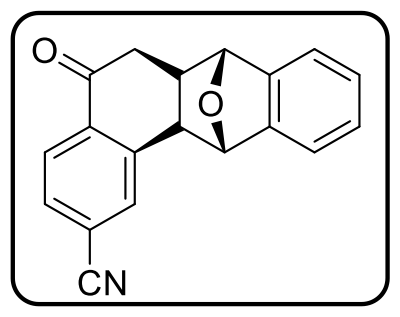

(rac)-5-oxo-5,6,6a,7,12,12a-hexahydro-7,12-epoxytetraphene-2-carb onitrile (3ka)

Yellow solid (29.9 mg, 52\%), ${ }^{1} \mathrm{H}$ NMR $\left(400 \mathrm{MHz}, \mathrm{CDCl}_{3}\right) \delta 7.93(\mathrm{~d}, J=$ $8.0 \mathrm{~Hz}, 1 \mathrm{H}), 7.82(\mathrm{~d}, J=0.8 \mathrm{~Hz}, 1 \mathrm{H}), 7.66\left(\mathrm{dd}, J_{1}=8.0 \mathrm{~Hz}, J_{2}=1.6 \mathrm{~Hz}\right.$, $1 \mathrm{H}), 7.42-7.38(\mathrm{~m}, 1 \mathrm{H}), 7.36-7.33(\mathrm{~m}, 1 \mathrm{H}), 7.30-7.26(\mathrm{~m}, 2 \mathrm{H}), 5.21$ $(\mathrm{s}, 1 \mathrm{H}), 4.98(\mathrm{~s}, 1 \mathrm{H}), 3.30(\mathrm{~d}, J=8.0 \mathrm{~Hz}, 1 \mathrm{H}), 3.01\left(\mathrm{dd}, J_{1}=15.2 \mathrm{~Hz}, J_{2}\right.$ $=2.4 \mathrm{~Hz}, 1 \mathrm{H}), 2.81\left(\mathrm{dd}, J_{1}=15.2 \mathrm{~Hz}, J_{2}=8.4 \mathrm{~Hz}, 1 \mathrm{H}\right), 2.60\left(\mathrm{td}, J_{1}=8.0 \mathrm{~Hz}, J_{2}=2.4 \mathrm{~Hz}, 1 \mathrm{H}\right) .{ }^{13} \mathrm{C}$ NMR $\left(100 \mathrm{MHz}, \mathrm{CDCl}_{3}\right) \delta 196.7,144.9,144.0,143.7,137.8,133.2,130.4,127.8,127.7,126.7$, 119.7, 119.6, 118.1, 116.8, 87.7, 86.4, 43.9, 42.2, 38.1. HRMS (ESI-TOF) (m/z): Calcd for $\mathrm{C}_{19} \mathrm{H}_{13} \mathrm{NNaO}_{2},\left([\mathrm{M}+\mathrm{Na}]^{+}\right), 310.0838$, found 310.0836 . 


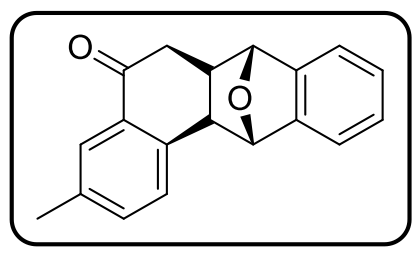

(rac)-3-methyl-6a,7,12,12a-tetrahydro-7,12-epoxytetraphen-5(6H)one (3la)

Yellow solid (41.9 mg, 76\%), ${ }^{1} \mathrm{H}$ NMR (400 MHz, $\left.\mathrm{CD}_{3} \mathrm{CN}\right) \delta 7.55-$ $7.51(\mathrm{~m}, 1 \mathrm{H}), 7.50-7.46(\mathrm{~m}, 2 \mathrm{H}), 7.45-7.42(\mathrm{~m}, 1 \mathrm{H}), 7.36-7.33(\mathrm{~m}$, $1 \mathrm{H}), 7.27-7.21(\mathrm{~m}, 2 \mathrm{H}), 5.14(\mathrm{~s}, 1 \mathrm{H}), 4.90(\mathrm{~s}, 1 \mathrm{H}), 3.23(\mathrm{~d}, J=8.0 \mathrm{~Hz}$ 1H), $2.80-2.77(\mathrm{~m}, 2 \mathrm{H}), 2.48-2.43(\mathrm{~m}, 1 \mathrm{H}), 2.38(\mathrm{~s}, 3 \mathrm{H}),{ }^{13} \mathrm{C} \mathrm{NMR}\left(100 \mathrm{MHz}, \mathrm{CDCl}_{3}\right) \delta 198.4$, $145.1,144.5,140.1,136.7,134.5,129.0,127.3,127.2,126.0,119.4,87.9,86.4,43.5,42.3,37.8$, 21.1. HRMS (ESI-TOF) (m/z): Calcd for $\mathrm{C}_{19} \mathrm{H}_{16} \mathrm{NaO}_{2}$, $\left([\mathrm{M}+\mathrm{Na}]^{+}\right), 299.1043$, found 299.1041 .

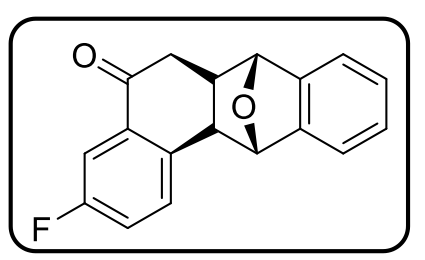

(rac)-3-fluoro-6a,7,12,12a-tetrahydro-7,12-epoxytetraphen-5(6H)-o ne (rac) (3ma)

Yellow solid (26.5 mg, 47\%), ${ }^{1} \mathrm{H}$ NMR (400 MHz, $\left.\mathrm{CD}_{3} \mathrm{CN}\right) \delta 7.58-$ $7.54(\mathrm{~m}, 1 \mathrm{H}), 7.44-7.39(\mathrm{~m}, 3 \mathrm{H}), 7.39-7.36(\mathrm{~m}, 1 \mathrm{H}), 7.28-7.23(\mathrm{~m}$, $2 \mathrm{H}), 5.19(\mathrm{~s}, 1 \mathrm{H}), 4.99(\mathrm{~s}, 1 \mathrm{H}), 3.42\left(\mathrm{dd}, J_{1}=8.0 \mathrm{~Hz}, J_{2}=2.4 \mathrm{~Hz}, 1 \mathrm{H}\right)$, $2.90-2.80(\mathrm{~m}, 2 \mathrm{H}), 2.52\left(\mathrm{td}, J_{1}=7.6 \mathrm{~Hz}, J_{2}=2.8 \mathrm{~Hz}, 1 \mathrm{H}\right) .{ }^{13} \mathrm{C} \mathrm{NMR}\left(100 \mathrm{MHz}, \mathrm{CD}_{3} \mathrm{CN}\right) \delta 198.0(J=$ $3.0 \mathrm{~Hz}), 161.3(J=244.5 \mathrm{~Hz}), 146.4,145.1,137.5(J=2.8 \mathrm{~Hz}), 131.6(J=15.3 \mathrm{~Hz}), 129.1,129.0$, 128.9, 128.1, 121.8, 120.5, 120.3, 87.2, 86.9, 42.3, 38.7, 37.8. HRMS (ESI-TOF) (m/z): Calcd for $\mathrm{C}_{18} \mathrm{H}_{13} \mathrm{FNaO}_{2},\left([\mathrm{M}+\mathrm{Na}]^{+}\right), 303.0792$, found 303.0789 .

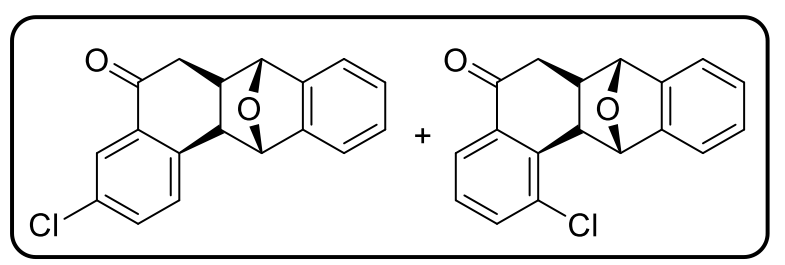

(rac)-3-chloro-6a,7,12,12a-tetrahydro-7,12-epoxytetraphen-5(6H)-one (3na) (rac))-1-chloro-6a,7,12,12a-tetrahydro-7,12-epoxytetraphen-5(6H)-one (3na')

(3na:3na' $=5.9: 1)$

Yellow solid (39.6 mg, 67\%), ${ }^{1} \mathrm{H}$ NMR (400 MHz, $\left.\mathrm{CD}_{3} \mathrm{CN}\right) \delta 7.71\left(\mathrm{dd}, J_{1}=17.6 \mathrm{~Hz}, J_{2}=8.0 \mathrm{~Hz}, 2 \mathrm{H}\right)$, $7.65-7.59(\mathrm{~m}, 0.34 \mathrm{H}), 7.47-7.33(\mathrm{~m}, 3.51 \mathrm{H}), 7.30-7.23(\mathrm{~m}, 2.34 \mathrm{H}), 5.20(\mathrm{~s}, 1 \mathrm{H}), 5.16(\mathrm{~s}, 0.17 \mathrm{H})$, $4.95(\mathrm{~s}, 1 \mathrm{H}), 4.91(\mathrm{~s}, 0.17 \mathrm{H}), 3.53(\mathrm{~d}, J=8.0 \mathrm{~Hz}, 1 \mathrm{H}), 3.27(\mathrm{~d}, J=7.6 \mathrm{~Hz}, 0.17 \mathrm{H}), 2.90-2.77(\mathrm{~m}$, $2.34 \mathrm{H}), 2.54-2.45(\mathrm{~m}, 1.17 \mathrm{H}) .{ }^{13} \mathrm{C} \mathrm{NMR}\left(100 \mathrm{MHz}, \mathrm{CD}_{3} \mathrm{CN}\right) \delta 198.3,198.0,146.5,145.3,145.2$, 143.3, 141.6, 137.6, 137.1, 134.9, 134.8, 133.9, 132.5, 128.8, 128.2, 128.1, 128.0, 125.3, 124.9, 120.6, $120.3,120.2,88.4,87.22,87.15,87.0,43.9,42.5,42.2,42.0,38.7,38.6$. HRMS (ESI-TOF) (m/z): Calcd for $\mathrm{C}_{18} \mathrm{H}_{13} \mathrm{ClNaO}_{2},\left([\mathrm{M}+\mathrm{Na}]^{+}\right), 319.0496$, found 319.0493 .

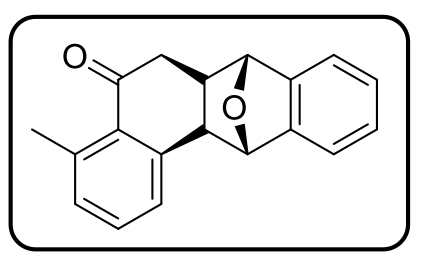

(rac)-4-methyl-6a,7,12,12a-tetrahydro-7,12-epoxytetraphen-5(6H)one (30a)

Yellow solid (19.4 mg, 35\%), ${ }^{1} \mathrm{H}$ NMR (400 MHz, $\left.\mathrm{CD}_{3} \mathrm{CN}\right) \delta 7.48-$ $7.42(\mathrm{~m}, 2 \mathrm{H}), 7.41-7.38(\mathrm{~m}, 1 \mathrm{H}), 7.37-7.33(\mathrm{~m}, 1 \mathrm{H}), 7.27-7.21(\mathrm{~m}$, $2 \mathrm{H}), 7.18(\mathrm{~d}, J=7.2 \mathrm{~Hz}, 1 \mathrm{H}), 5.16(\mathrm{~s}, 1 \mathrm{H}), 4.89(\mathrm{~s}, 1 \mathrm{H}), 3.25(\mathrm{~d}, J=$ $8.0 \mathrm{~Hz}, 1 \mathrm{H}), 2.78-2.73(\mathrm{~m}, 2 \mathrm{H}), 2.49(\mathrm{~s}, 3 \mathrm{H}), 2.43\left(\mathrm{td}, J_{1}=7.2 \mathrm{~Hz}, J_{2}\right.$ $=2.8 \mathrm{~Hz}, 1 \mathrm{H}) .{ }^{13} \mathrm{C} \mathrm{NMR}\left(100 \mathrm{MHz}, \mathrm{CD}_{3} \mathrm{CN}\right) \delta 200.7,146.5,145.7,145.0,137.8,134.9,132.9,130.6$, 128.4, 127.9, 120.5, 120.2, 88.7, 86.8, 45.3, 45.1, 38.8, 30.8, 21.0. HRMS (ESI-TOF) (m/z): Calcd for $\mathrm{C}_{19} \mathrm{H}_{16} \mathrm{NaO}_{2},\left([\mathrm{M}+\mathrm{Na}]^{+}\right), 299.1043$, found 299.1041 . 


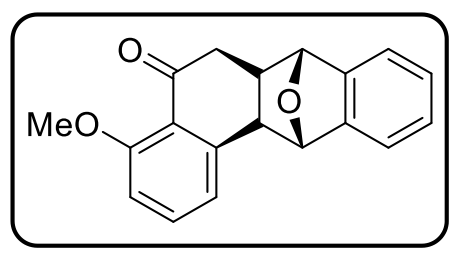

(rac)-4-methoxy-6a,7,12,12a-tetrahydro-7,12-epoxytetraphen-5( 6H)-one (3pa)

Yellow solid (36.1 mg, 62\%), ${ }^{1} \mathrm{H}$ NMR (400 MHz, $\left.\mathrm{CD}_{3} \mathrm{CN}\right) \delta 7.53(\mathrm{t}$, $J=8.0 \mathrm{~Hz}, 1 \mathrm{H}), 7.45-7.41(\mathrm{~m}, 1 \mathrm{H}), 7.37-7.33(\mathrm{~m}, 1 \mathrm{H}), 7.27-$ $7.21(\mathrm{~m}, 2 \mathrm{H}), 7.12(\mathrm{~d}, J=7.6 \mathrm{~Hz}, 1 \mathrm{H}), 6.97(\mathrm{~d}, J=8.4 \mathrm{~Hz}, 1 \mathrm{H}), 5.15$ $(\mathrm{s}, 1 \mathrm{H}), 4.93(\mathrm{~s}, 1 \mathrm{H}), 3.81(\mathrm{~s}, 3 \mathrm{H}), 3.21(\mathrm{~d}, J=7.6 \mathrm{~Hz}, 1 \mathrm{H}), 2.76\left(\mathrm{dd}, J_{1}=13.2 \mathrm{~Hz}, J_{2}=8.0 \mathrm{~Hz}, 1 \mathrm{H}\right)$, $2.68\left(\mathrm{dd}, J_{1}=13.6 \mathrm{~Hz}, J_{2}=2.4 \mathrm{~Hz}, 1 \mathrm{H}\right), 2.41\left(\mathrm{td}, J_{1}=8.0 \mathrm{~Hz}, J_{2}=2.4 \mathrm{~Hz}, 1 \mathrm{H}\right) .{ }^{13} \mathrm{C} \mathrm{NMR}(100 \mathrm{MHz}$, $\left.\mathrm{CD}_{3} \mathrm{CN}\right) \delta 197.1,157.9,146.5,146.3,145.8,134.4,127.9,125.7,122.4,122.3,120.5,120.2,110.8$, 88.6, 86.9, 56.4, 45.4, 45.1, 38.9. HRMS (ESI-TOF) $(\mathrm{m} / \mathrm{z})$ : Calcd for $\mathrm{C}_{19} \mathrm{H}_{16} \mathrm{NaO}_{3}$, $\left([\mathrm{M}+\mathrm{Na}]^{+}\right)$, 315.0992 , found 319.0988 .

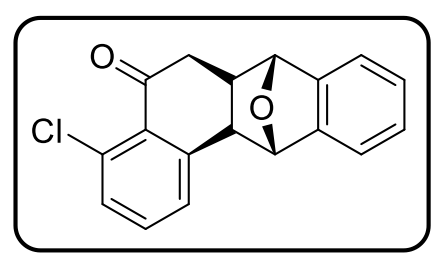

(rac)-4-chloro-6a,7,12,12a-tetrahydro-7,12-epoxytetraphen-5(6H)one (3qa)

Yellow solid (25.7 mg, 43\%), ${ }^{1} \mathrm{H}$ NMR (400 MHz, $\left.\mathrm{CD}_{3} \mathrm{CN}\right) \delta 7.55-$ $7.51(\mathrm{~m}, 2 \mathrm{H}), 7.45-7.42(\mathrm{~m}, 1 \mathrm{H}), 7.42-7.39(\mathrm{~m}, 1 \mathrm{H}), 7.38-7.35$ (m, 1H), $7.27-7.23(\mathrm{~m}, 2 \mathrm{H}), 5.18(\mathrm{~s}, 1 \mathrm{H}), 4.94(\mathrm{~s}, 1 \mathrm{H}), 3.29(\mathrm{~d}, J=$ $7.6 \mathrm{~Hz}, 1 \mathrm{H}), 2.83-2.78(\mathrm{~m}, 2 \mathrm{H}), 2.47\left(\mathrm{td}, J_{1}=7.6 \mathrm{~Hz}, J_{2}=3.2 \mathrm{~Hz}, 1 \mathrm{H}\right) .{ }^{13} \mathrm{C} \mathrm{NMR}\left(100 \mathrm{MHz}, \mathrm{CD}_{3} \mathrm{CN}\right)$ $\delta$ 197.2, 146.9, 146.3, 145.5, 133.8, 133.6, 131.1, 130.1, 129.7, 128.1, 128.0, 120.6, 120.3, 88.4, 86.7, 45.2, 44.9, 39.1. HRMS (ESI-TOF) (m/z): Calcd for $\mathrm{C}_{18} \mathrm{H}_{13} \mathrm{ClNaO}_{2}$, $\left([\mathrm{M}+\mathrm{Na}]^{+}\right), 319.0496$, found 319.0489 .

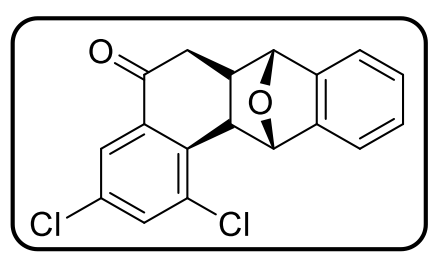

(rac)-1,3-dichloro-6a,7,12,12a-tetrahydro-7,12-epoxytetraphen-5( 6H)-one (3ra)

Yellow solid (48.7 mg, 74\%), ${ }^{1} \mathrm{H}$ NMR (400 MHz, $\left.\mathrm{CDCl}_{3}\right) \delta 7.78(\mathrm{~d}$, $J=2.0 \mathrm{~Hz}, 1 \mathrm{H}), 7.67$ (d, $J=2.4 \mathrm{~Hz}, 1 \mathrm{H}), 7.44-7.40$ (m, 1H), $7.36-$ $7.32(\mathrm{~m}, 1 \mathrm{H}), 7.30-7.26(\mathrm{~m}, 1 \mathrm{H}), 7.26-7.22(\mathrm{~m}, 1 \mathrm{H}), 5.20(\mathrm{~s}, 1 \mathrm{H})$, $5.00(\mathrm{~s}, 1 \mathrm{H}), 3.54(\mathrm{~d}, J=8.0 \mathrm{~Hz}, 1 \mathrm{H}), 2.99\left(\mathrm{dd}, J_{1}=15.6 \mathrm{~Hz}, J_{2}=2.0 \mathrm{~Hz}, 1 \mathrm{H}\right), 2.79\left(\mathrm{dd}, J_{1}=15.6 \mathrm{~Hz}\right.$, $\left.J_{2}=8.8 \mathrm{~Hz}, 1 \mathrm{H}\right), 2.56\left(\mathrm{td}, J_{1}=8.4 \mathrm{~Hz}, J_{2}=2.0 \mathrm{~Hz}, 1 \mathrm{H}\right) .{ }^{13} \mathrm{C} \mathrm{NMR}\left(100 \mathrm{MHz}, \mathrm{CDCl}_{3}\right) \delta 195.9,145.0$, 143.9, 139.0, 137.1, 135.1, 133.7, 127.7, 127.5, 124.9, 119.8, 119.6, 119.4, 86.5, 86.3, 41.2, 41.1, 37.9. HRMS (ESI-TOF) (m/z): Calcd for $\mathrm{C}_{18} \mathrm{H}_{12} \mathrm{Cl}_{2} \mathrm{NaO}_{2}$, ([M+Na] $\left.]^{+}\right), 353.0107$, found 353.0104 .

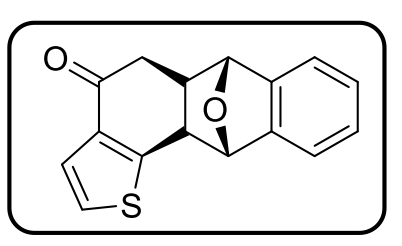

(rac)-5a,6,11,11a-tetrahydro-6,11-epoxyanthra[1,2-b]thiophen-4(5H) -one (3sa)

Yellow solid (45.4 mg, 85\%), ${ }^{1} \mathrm{H}$ NMR (400 MHz, $\left.\mathrm{CD}_{3} \mathrm{CN}\right) \delta 7.83(\mathrm{~d}, J$ $=4.8 \mathrm{~Hz}, 1 \mathrm{H}), 7.46-7.43(\mathrm{~m}, 1 \mathrm{H}), 7.37-7.34(\mathrm{~m}, 1 \mathrm{H}), 7.33(\mathrm{~d}, J=5.2$ $\mathrm{Hz}, 1 \mathrm{H}), 7.27-7.22(\mathrm{~m}, 2 \mathrm{H}), 5.16(\mathrm{~s}, 1 \mathrm{H}), 5.13(\mathrm{~s}, 1 \mathrm{H}), 3.28(\mathrm{~d}, J=8.0$ $\mathrm{Hz}, 1 \mathrm{H}), 2.92\left(\mathrm{dd}, J_{1}=16.8 \mathrm{~Hz}, J_{2}=8.8 \mathrm{~Hz}, 1 \mathrm{H}\right), 2.81\left(\mathrm{dd}, J_{1}=17.2 \mathrm{~Hz}, J_{2}=3.6 \mathrm{~Hz}, 1 \mathrm{H}\right), 2.56-2.50$ (m, 1H). ${ }^{13} \mathrm{C}$ NMR $\left(100 \mathrm{MHz}, \mathrm{CD}_{3} \mathrm{CN}\right) \delta$ 191.6, 152.2, 146.6, 145.4, 137.0, 134.6, 129.7, 128.0, 127.9, 120.6, 120.1, 87.3, 86.4, 42.1, 42.0, 39.6. HRMS (ESI-TOF) $(\mathrm{m} / \mathrm{z})$ : Calcd for $\mathrm{C}_{16} \mathrm{H}_{12} \mathrm{NaO}_{2} \mathrm{~S}$, ([M + $\mathrm{Na}^{+}$), 291.0450, found 291.0444. 


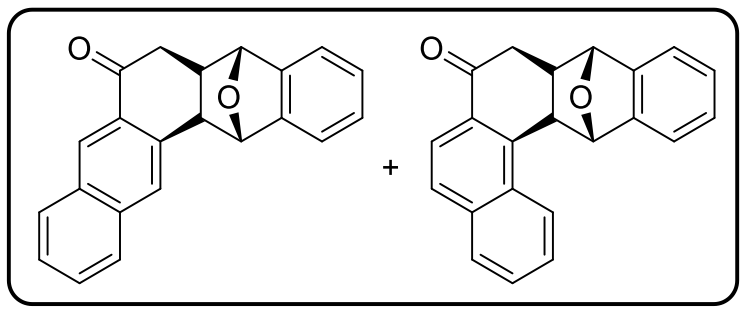

(rac)-5a,6,13b,14-tetrahydro-5,14-epoxypentaphen-7(5H)-one (3ta)

(rac)-8a,9,14,14a-tetrahydro-9,14-epoxybenzo[a]tetraphen-7(8H)-one (3ta')

(3ta:3ta' = 1.3:1)

White solid (28.7 mg, 46\%), ${ }_{1}^{1} \mathrm{H}$ NMR $\left(600 \mathrm{MHz},\left(\mathrm{CD}_{3}\right)_{2} \mathrm{CO}\right) \delta 8.31(\mathrm{~d}, J=8.4 \mathrm{~Hz}, 0.78 \mathrm{H}), 8.29(\mathrm{~s}, 1 \mathrm{H})$, $8.13(\mathrm{~s}, 1 \mathrm{H}), 8.06(\mathrm{~d}, J=8.4 \mathrm{~Hz}, 1 \mathrm{H}), 8.03(\mathrm{~d}, J=8.4 \mathrm{~Hz}, 0.78 \mathrm{H}), 7.96(\mathrm{~d}, J=8.4 \mathrm{~Hz}, 1 \mathrm{H}), 7.92-7.83$ $(\mathrm{m}, 1.56 \mathrm{H}), 7.77(\mathrm{t}, J=7.8 \mathrm{~Hz}, 0.78 \mathrm{H}), 7.71(\mathrm{t}, J=7.2 \mathrm{~Hz}, 0.78 \mathrm{H}), 7.61(\mathrm{t}, J=7.2 \mathrm{~Hz}, 1 \mathrm{H}), 7.58(\mathrm{~d}, J=$ $6.6 \mathrm{~Hz}, 0.78 \mathrm{H}), 7.54(\mathrm{t}, J=7.2 \mathrm{~Hz}, 1 \mathrm{H}), 7.49(\mathrm{~d}, J=6.6 \mathrm{~Hz}, 1 \mathrm{H}), 7.45(\mathrm{~d}, J=6.6 \mathrm{~Hz}, 0.78 \mathrm{H}), 7.41(\mathrm{~d}, J$ $=6.0 \mathrm{~Hz}, 1 \mathrm{H}), 7.32-7.26(\mathrm{~m}, 1.56 \mathrm{H}), 7.26-7.21(\mathrm{~m}, 2 \mathrm{H}), 5.30(\mathrm{~s}, 0.78 \mathrm{H}), 5.29(\mathrm{~s}, 1 \mathrm{H}), 5.04(\mathrm{~s}, 1 \mathrm{H})$, $4.89(\mathrm{~s}, 0.78 \mathrm{H}), 4.01(\mathrm{~d}, J=7.8 \mathrm{~Hz}, 0.78 \mathrm{H}), 3.48(\mathrm{~d}, J=7.8 \mathrm{~Hz}, 1 \mathrm{H}), 3.00-2.85(\mathrm{~m}, 3.56 \mathrm{H}), 2.67(\mathrm{t}, J$ $=7.8 \mathrm{~Hz}, 0.78 \mathrm{H}), 2.62(\mathrm{~d}, J=7.8 \mathrm{~Hz}, 1 \mathrm{H}) .{ }^{13} \mathrm{C} \mathrm{NMR}\left(150 \mathrm{MHz},\left(\mathrm{CD}_{3}\right)_{2} \mathrm{CO}\right) \delta 198.3,197.8,146.9$, 146.7, 145.9, 145.5, 141.4, 140.5, 137.0, 136.9, 134.3, 133.1, 132.5, 132.2, 130.1, 129.0, 128.8, 128.7, $128.3,128.2,128.0,127.9,127.8,127.7,127.1,126.1,125.5,122.6,120.4,120.22,120.15,120.1,89.5$, 87.6, 87.4, 87.1, 44.7, 43.3, 41.9, 40.7, 39.3, 38.5. HRMS (ESI-TOF) (m/z): Calcd for $\mathrm{C}_{22} \mathrm{H}_{16} \mathrm{NaO}_{2}$, $\left([\mathrm{M}+\mathrm{Na}]^{+}\right), 335.1043$, found 335.1034 .

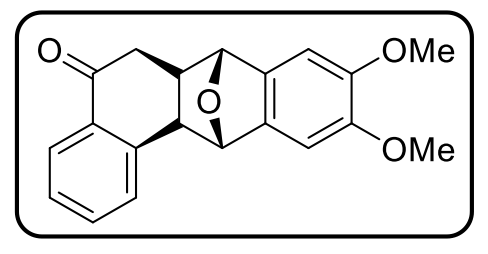

(rac)-9,10-dimethoxy-6a,7,12,12a-tetrahydro-7,12-epoxytetrap hen-5(6H)-one (3ab)

Yellow solid (31.0 mg, 48\%), ${ }^{1} \mathrm{H}$ NMR (400 $\left.\mathrm{MHz}, \mathrm{CD}_{3} \mathrm{CN}\right) \delta$ $7.70\left(\mathrm{dd}, J_{1}=7.6 \mathrm{~Hz}, J_{2}=1.2 \mathrm{~Hz}, 1 \mathrm{H}\right), 7.63\left(\mathrm{td}, J_{1}=7.6 \mathrm{~Hz}, J_{2}=\right.$ $1.2 \mathrm{~Hz}, 1 \mathrm{H}), 7.56\left(\mathrm{dd}, J_{1}=7.6 \mathrm{~Hz}, J_{2}=1.2 \mathrm{~Hz}, 1 \mathrm{H}\right), 7.37\left(\mathrm{td}, J_{1}=\right.$ $\left.7.2 \mathrm{~Hz}, J_{2}=1.2 \mathrm{~Hz}, 1 \mathrm{H}\right), 7.10(\mathrm{~s}, 1 \mathrm{H}), 7.00(\mathrm{~s}, 1 \mathrm{H}), 5.08(\mathrm{~s}, 1 \mathrm{H}), 4.83(\mathrm{~s}, 1 \mathrm{H}), 3.81(\mathrm{~s}, 3 \mathrm{H}), 3.79(\mathrm{~s}, 3 \mathrm{H})$, $3.18(\mathrm{~d}, J=7.6 \mathrm{~Hz}, 1 \mathrm{H}), 2.79-2.76(\mathrm{~m}, 2 \mathrm{H}), 2.41-2.36(\mathrm{~m}, 1 \mathrm{H}) .{ }^{13} \mathrm{C} \mathrm{NMR}\left(100 \mathrm{MHz}, \mathrm{CD}_{3} \mathrm{CN}\right) \delta$ 199.1, 149.32, 149.27, 144.7, 138.7, 137.6, 135.7, 134.2, 130.4, 127.4, 125.7, 105.7, 105.6, 88.9, 87.4, 56.6, 44.9, 42.8, 39.0. HRMS (ESI-TOF) (m/z): Calcd for $\mathrm{C}_{20} \mathrm{H}_{18} \mathrm{NaO}_{4},\left([\mathrm{M}+\mathrm{Na}]^{+}\right), 345.1097$, found 345.1093 .

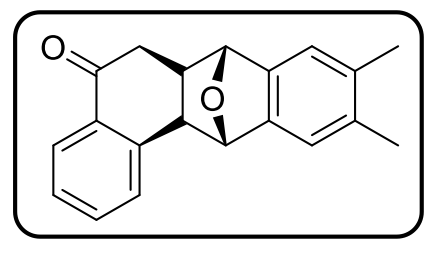

(rac)-9,10-dimethyl-6a,7,12,12a-tetrahydro-7,12-epoxytetraphen5(6H)-one (3ac)

Yellow solid (38.7 mg, 67\%), ${ }^{1} \mathrm{H}$ NMR $\left(400 \mathrm{MHz}, \mathrm{CD}_{3} \mathrm{CN}\right) \delta 7.70(\mathrm{~d}$, $J=7.6 \mathrm{~Hz}, 1 \mathrm{H}), 7.66-7.61(\mathrm{~m}, 1 \mathrm{H}), 7.58(\mathrm{~d}, J=7.6 \mathrm{~Hz}, 1 \mathrm{H}), 7.40-$ $7.35(\mathrm{~m}, 1 \mathrm{H}), 7.21(\mathrm{~s}, 1 \mathrm{H}), 7.13(\mathrm{~s}, 1 \mathrm{H}), 5.08(\mathrm{~s}, 1 \mathrm{H}), 4.84(\mathrm{~s}, 1 \mathrm{H})$, $3.23(\mathrm{~d}, J=8.0 \mathrm{~Hz}, 1 \mathrm{H}), 2.81-2.77(\mathrm{~m}, 2 \mathrm{H}), 2.46-2.41(\mathrm{~m}, 1 \mathrm{H}), 2.27(\mathrm{~s}, 3 \mathrm{H}), 2.26(\mathrm{~s}, 3 \mathrm{H}) .{ }^{13} \mathrm{C} \mathrm{NMR}$ $\left(100 \mathrm{MHz}, \mathrm{CD}_{3} \mathrm{CN}\right) \delta 199.2,144.7,144.5,143.4,136.1,136.0,135.8,134.2,130.5,127.5,125.7$, 121.7, 121.3, 88.6, 87.1, 44.8, 42.8, 39.0, 20.0. HRMS (ESI-TOF) (m/z): Calcd for $\mathrm{C}_{20} \mathrm{H}_{18} \mathrm{NaO}_{2}$, ([M + $\mathrm{Na}]^{+}$), 313.1199, found 313.1193 . 


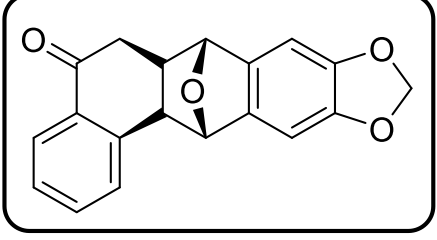

(rac)-6a,7,13,13a-tetrahydro-7,13-epoxytetrapheno $[9,10-d][1,3] \mathrm{di}$ oxol-5(6H)-one (3ad)

Yellow solid (43.3 mg, 71\%), ${ }^{1} \mathrm{H}$ NMR (400 $\left.\mathrm{MHz}, \mathrm{CD}_{3} \mathrm{CN}\right) \delta 7.70$ $\left(\mathrm{dd}, J_{1}=8.0 \mathrm{~Hz}, J_{2}=1.2 \mathrm{~Hz}, 1 \mathrm{H}\right), 7.65-7.60(\mathrm{~m}, 1 \mathrm{H}), 7.57(\mathrm{~d}, J=$ $7.2 \mathrm{~Hz}, 1 \mathrm{H}), 7.40-7.34(\mathrm{~m}, 1 \mathrm{H}), 6.97(\mathrm{~s}, 1 \mathrm{H}), 6.88(\mathrm{~s}, 1 \mathrm{H}), 5.97(\mathrm{~d}$, $J=0.8 \mathrm{~Hz}, 1 \mathrm{H}), 5.94(\mathrm{~d}, J=0.8 \mathrm{~Hz}, 1 \mathrm{H}), 5.06(\mathrm{~s}, 1 \mathrm{H}), 4.81(\mathrm{~s}, 1 \mathrm{H}), 3.21(\mathrm{~d}, J=8.0 \mathrm{~Hz}, 1 \mathrm{H}), 2.80-$ $2.76(\mathrm{~m}, 2 \mathrm{H}), 2.45-2.39(\mathrm{~m}, 1 \mathrm{H}) .{ }^{13} \mathrm{C} \mathrm{NMR}\left(100 \mathrm{MHz}, \mathrm{CD}_{3} \mathrm{CN}\right) \delta 199.1,147.6,147.5,144.6,140.3$, $139.1,135.7,134.2,130.4,127.5,125.7,102.6,102.4,88.69,88.65,87.2,44.6,42.7,38.7$. HRMS (ESI-TOF) $(\mathrm{m} / \mathrm{z})$ : Calcd for $\mathrm{C}_{19} \mathrm{H}_{14} \mathrm{NaO}_{4},\left([\mathrm{M}+\mathrm{Na}]^{+}\right), 329.0784$, found 329.0782 .

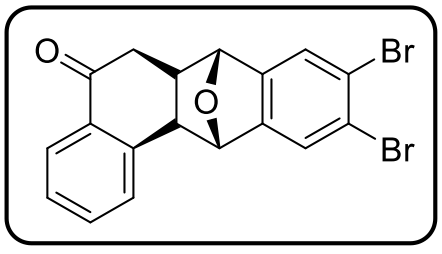

(rac)-9,10-dibromo-6a,7,12,12a-tetrahydro-7,12-epoxytetraphen5(6H)-one (3ae)

White solid (51.6 mg, 62\%), ${ }^{1} \mathrm{H}$ NMR (400 MHz, $\left.\mathrm{CDCl}_{3}\right) \delta 7.84$ (dd, $\left.J_{1}=7.6 \mathrm{~Hz}, J_{2}=1.2 \mathrm{~Hz}, 1 \mathrm{H}\right), 7.65(\mathrm{~s}, 1 \mathrm{H}), 7.63-7.58(\mathrm{~m}, 2 \mathrm{H}), 7.44$ $(\mathrm{d}, J=7.6 \mathrm{~Hz}, 1 \mathrm{H}), 7.38(\mathrm{t}, J=7.6 \mathrm{~Hz}, 1 \mathrm{H}), 5.15(\mathrm{~s}, 1 \mathrm{H}), 4.95$ (s, $1 \mathrm{H}), 3.26(\mathrm{~d}, J=7.6 \mathrm{~Hz}, 1 \mathrm{H}), 2.90\left(\mathrm{dd}, J_{1}=15.2 \mathrm{~Hz}, J_{2}=2.8 \mathrm{~Hz}, 1 \mathrm{H}\right), 2.83\left(\mathrm{dd}, J_{1}=15.2 \mathrm{~Hz}, J_{2}=8.0\right.$ $\mathrm{Hz}, 1 \mathrm{H}), 2.55\left(\mathrm{td}, J_{1}=8.0 \mathrm{~Hz}, J_{2}=2.8 \mathrm{~Hz}, 1 \mathrm{H}\right) .{ }^{13} \mathrm{C} \mathrm{NMR}\left(100 \mathrm{MHz}, \mathrm{CDCl}_{3}\right) \delta 197.6,146.1,145.4$, 142.1 , 134.7, 133.8, 129.0, 127.3, 126.0, 125.0, 123.34, 123.29, 87.2, 85.8, 43.6, 41.9, 37.6. HRMS (ESI-TOF) (m/z): Calcd for $\mathrm{C}_{18} \mathrm{H}_{12} \mathrm{Br}_{2} \mathrm{NaO}_{2}$, $\left([\mathrm{M}+\mathrm{Na}]^{+}\right), 440.9096$, found 440.9091 .

\section{General procedure for preparation of 5 .}

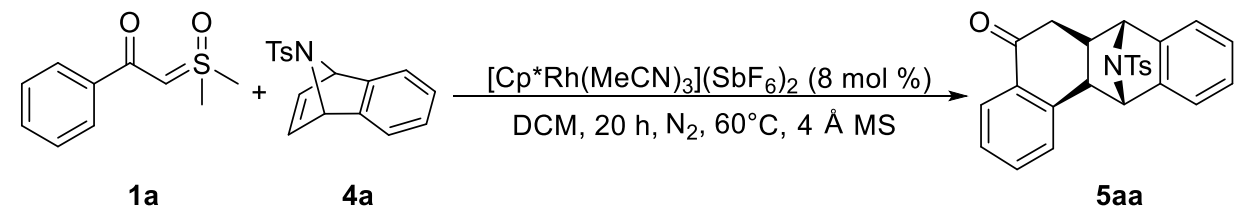

Representative Synthesis of Product 5. A pressure tube was charged with $4 \mathbf{a}(59.4 \mathrm{mg}, 0.2 \mathrm{mmol})$, 1a (58.8 mg, 1.5 equiv), $\left[\mathrm{Cp} * \mathrm{Rh}(\mathrm{MeCN})_{3}\right]\left(\mathrm{SbF}_{6}\right)_{2}(13.3 \mathrm{mg}, 8 \mathrm{~mol} \%), 4 \AA \mathrm{MS}(100 \mathrm{mg})$, and anhydrous DCM $(2.0 \mathrm{~mL})$. The reaction mixture was stirred at $60{ }^{\circ} \mathrm{C}$ for $20 \mathrm{~h}$ under $\mathrm{N}_{2}$. After the reaction was complete as indicated by TLC analysis, the solvent was removed under reduced pressure and the residue was purified by silica gel chromatography using petroleum ether/ethyl acetate $5: 1(\mathrm{v} / \mathrm{v})$ to give the corresponding product $\mathbf{5} \mathbf{a a}(68.1 \mathrm{mg}, 82 \%)$.

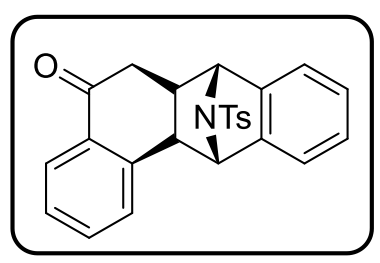

(rac)-13-tosyl-6a,7,12,12a-tetrahydro-7,12-epiminotetraphen-5(6H)-o ne (5aa)

Yellow solid (68.1 mg, 82\%), ${ }^{1} \mathrm{H}$ NMR (400 MHz, $\left.\mathrm{CDCl}_{3}\right) \delta 7.83$ (dd, $J_{1}$ $\left.=8.0 \mathrm{~Hz}, J_{2}=1.6 \mathrm{~Hz}, 1 \mathrm{H}\right), 7.55\left(\mathrm{td}, J_{1}=7.6 \mathrm{~Hz}, J_{2}=1.6 \mathrm{~Hz}, 1 \mathrm{H}\right), 7.43(\mathrm{~d}$, $J=7.6 \mathrm{~Hz}, 1 \mathrm{H}), 7.35\left(\mathrm{td}, J_{1}=7.6 \mathrm{~Hz}, J_{2}=0.8 \mathrm{~Hz}, 1 \mathrm{H}\right), 7.27-7.24(\mathrm{~m}$, 2H), $7.16-7.13(\mathrm{~m}, 1 \mathrm{H}), 7.08-6.99(\mathrm{~m}, 3 \mathrm{H}), 6.93(\mathrm{~d}, J=8.0 \mathrm{~Hz}, 2 \mathrm{H}), 4.84(\mathrm{~s}, 1 \mathrm{H}), 4.73(\mathrm{~s}, 1 \mathrm{H}), 3.14$ $(\mathrm{d}, J=8.4 \mathrm{~Hz}, 1 \mathrm{H}), 2.96\left(\mathrm{dd}, J_{1}=15.2 \mathrm{~Hz}, J_{2}=3.6 \mathrm{~Hz}, 1 \mathrm{H}\right), 2.82\left(\mathrm{dd}, J_{1}=15.6 \mathrm{~Hz}, J_{2}=8.4 \mathrm{~Hz}, 1 \mathrm{H}\right)$, $2.47\left(\mathrm{td}, J_{1}=8.0 \mathrm{~Hz}, J_{2}=3.6 \mathrm{~Hz}, 1 \mathrm{H}\right), 2.25(\mathrm{~s}, 3 \mathrm{H}) .{ }^{13} \mathrm{C} \mathrm{NMR}\left(100 \mathrm{MHz}, \mathrm{CDCl}_{3}\right) \delta 196.8,143.3,143.2$, 142.8, 141.4, 135.0, 134.6, 133.6, 129.1, 129.0, 127.9, 127.4, 127.3, 127.2, 125.9, 120.5, 120.4, 72.4, 70.4, 44.1, 42.0, 38.6, 21.5. HRMS (ESI-TOF) (m/z): Calcd for $\mathrm{C}_{25} \mathrm{H}_{21} \mathrm{NNaO}_{3} \mathrm{~S},\left([\mathrm{M}+\mathrm{Na}]^{+}\right), 438.1134$, found 438.1128 . 


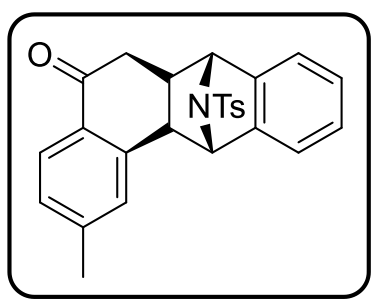

(rac)-2-methyl-13-tosyl-6a,7,12,12a-tetrahydro-7,12-epiminotetraphe n-5(6H)-one (5ba)

Yellow solid (72.8 mg, 85\%), ${ }^{1} \mathrm{H}$ NMR $\left(400 \mathrm{MHz}, \mathrm{CDCl}_{3}\right) \delta 7.76(\mathrm{~d}, J=$ $8.0 \mathrm{~Hz}, 1 \mathrm{H}), 7.29-7.26(\mathrm{~m}, 2 \mathrm{H}), 7.25-7.23(\mathrm{~m}, 1 \mathrm{H}), 7.18-7.14(\mathrm{~m}$, 2H), $7.08-6.99(\mathrm{~m}, 3 \mathrm{H}), 6.93$ (d, $J=8.4 \mathrm{~Hz}, 2 \mathrm{H}), 4.81$ (s, 1H), 4.80 (s, $1 \mathrm{H}), 3.09(\mathrm{~d}, J=8.0 \mathrm{~Hz}, 1 \mathrm{H}), 2.94\left(\mathrm{dd}, J_{1}=15.6 \mathrm{~Hz}, J_{2}=4.4 \mathrm{~Hz}, 1 \mathrm{H}\right)$, $2.83\left(\mathrm{dd}, J_{1}=15.2 \mathrm{~Hz}, J_{2}=8.0 \mathrm{~Hz}, 1 \mathrm{H}\right), 2.49-2.45(\mathrm{~m}, 1 \mathrm{H}), 2.43(\mathrm{~s}, 3 \mathrm{H}), 2.27(\mathrm{~s}, 3 \mathrm{H}) .{ }^{13} \mathrm{C} \mathrm{NMR}(100$ $\left.\mathrm{MHz}_{,} \mathrm{CDCl}_{3}\right) \delta 196.6,144.3,143.22,143.16,142.9,141.5,135.0,132.1,129.4,129.1,128.2,127.9$, 127.3, 126.1, 120.5, 120.3, 72.3, 70.4, 44.0, 41.9, 38.5, 21.9, 21.5. HRMS (ESI-TOF) (m/z): Calcd for $\mathrm{C}_{26} \mathrm{H}_{23} \mathrm{NNaO}_{3} \mathrm{~S},\left([\mathrm{M}+\mathrm{Na}]^{+}\right), 452.1291$, found 452.1287 .

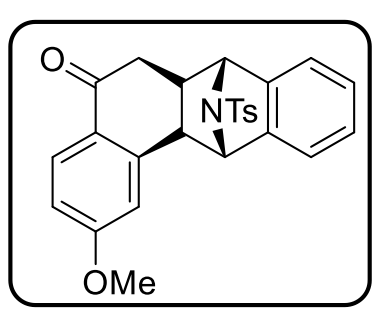

(rac)-2-methoxy-13-tosyl-6a,7,12,12a-tetrahydro-7,12-epiminotetraph en-5(6H)-one (5ca)

Yellow solid (54.4 mg, 61\%), ${ }^{1} \mathrm{H}$ NMR (400 $\left.\mathrm{MHz}, \mathrm{CDCl}_{3}\right) \delta 7.86-7.81$ (m, 1H), $7.29-7.25(\mathrm{~m}, 2 \mathrm{H}), 7.17-7.12(\mathrm{~m}, 1 \mathrm{H}), 7.07-6.98(\mathrm{~m}, 3 \mathrm{H})$, $6.93(\mathrm{~d}, J=8.4 \mathrm{~Hz}, 2 \mathrm{H}), 6.89-6.85(\mathrm{~m}, 2 \mathrm{H}), 4.82-4.79(\mathrm{~m}, 2 \mathrm{H}), 3.89(\mathrm{~s}$ $3 \mathrm{H}), 3.07(\mathrm{~d}, J=8.0 \mathrm{~Hz}, 1 \mathrm{H}), 2.90\left(\mathrm{dd}, J_{1}=15.6 \mathrm{~Hz}, J_{2}=4.4 \mathrm{~Hz}, 1 \mathrm{H}\right)$, $2.81\left(\mathrm{dd}, J_{1}=15.6 \mathrm{~Hz}, J_{2}=8.0 \mathrm{~Hz}, 1 \mathrm{H}\right), 2.48-2.41(\mathrm{~m}, 1 \mathrm{H}), 2.26(\mathrm{~s}, 3 \mathrm{H}) .{ }^{13} \mathrm{C} \mathrm{NMR}\left(100 \mathrm{MHz}, \mathrm{CDCl}_{3}\right)$ $\delta 195.5,163.8,143.7,143.24,143.18,142.9,135.1,129.1,128.4,127.9,127.33,127.28,120.5,120.3$, 113.6, 113.0, 72.3, 70.5, 55.7, 44.3, 41.7, 38.6, 21.5. HRMS (ESI-TOF) (m/z): Calcd for $\mathrm{C}_{26} \mathrm{H}_{23} \mathrm{NNaO}_{4} \mathrm{~S},\left([\mathrm{M}+\mathrm{Na}]^{+}\right), 468.1240$, found 468.1238 .

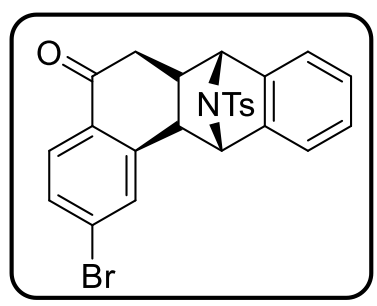

(rac)-2-bromo-13-tosyl-6a,7,12,12a-tetrahydro-7,12-epiminotetraphe n-5(6H)-one (5da)

Yellow solid $(80.7 \mathrm{mg}, 82 \%),{ }^{1} \mathrm{H}$ NMR $\left(400 \mathrm{MHz}, \mathrm{CDCl}_{3}\right) \delta 7.71(\mathrm{~d}, J=$ $8.4 \mathrm{~Hz}, 1 \mathrm{H}), 7.60(\mathrm{~d}, J=1.6 \mathrm{~Hz}, 1 \mathrm{H}), 7.49\left(\mathrm{dd}, J_{1}=8.4 \mathrm{~Hz}, J_{2}=2.0 \mathrm{~Hz}\right.$, $1 \mathrm{H}), 7.27-7.26(\mathrm{~m}, 1 \mathrm{H}), 7.25-7.24(\mathrm{~m}, 1 \mathrm{H}), 7.16-7.13(\mathrm{~m}, 1 \mathrm{H}), 7.08$ - $6.99(\mathrm{~m}, 3 \mathrm{H}), 6.94(\mathrm{~d}, J=8.0 \mathrm{~Hz}, 2 \mathrm{H}), 4.83(\mathrm{~s}, 1 \mathrm{H}), 4.73(\mathrm{~s}, 1 \mathrm{H}), 3.09$ $(\mathrm{d}, J=8.0 \mathrm{~Hz}, 1 \mathrm{H}), 2.96\left(\mathrm{dd}, J_{1}=15.6 \mathrm{~Hz}, J_{2}=3.6 \mathrm{~Hz}, 1 \mathrm{H}\right), 2.80\left(\mathrm{dd}, J_{1}=15.6 \mathrm{~Hz}, J_{2}=8.0 \mathrm{~Hz}, 1 \mathrm{H}\right)$, $2.47\left(\mathrm{td}, J_{1}=8.4 \mathrm{~Hz}, J_{2}=3.6 \mathrm{~Hz}, 1 \mathrm{H}\right), 2.27(\mathrm{~s}, 3 \mathrm{H}) .{ }^{13} \mathrm{C} \mathrm{NMR}\left(100 \mathrm{MHz}, \mathrm{CDCl}_{3}\right) \delta 195.7,143.4,143.3$, $143.1,142.4,134.9,133.4,131.9,130.7,129.1$, 128.2, 127.9, 127.7, 127.52, 127.47, 120.6, 120.5, 72.2, 70.3, 43.9, 41.9, 38.8, 21.5. HRMS (ESI-TOF) (m/z): Calcd for $\mathrm{C}_{25} \mathrm{H}_{20} \mathrm{BrNNaO}_{3} \mathrm{~S}$, $\left([\mathrm{M}+\mathrm{Na}]^{+}\right)$, 516.0239 , found 516.0241

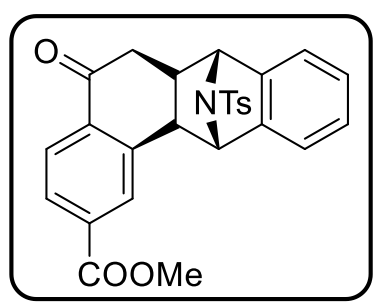

(rac)-5-oxo-13-tosyl-5,6,6a,7,12,12a-hexahydro-7,12-epiminotetraphe n-2-yl acetate (5ea)

Yellow solid (54.7 mg, 58\%), ${ }^{1} \mathrm{H}$ NMR (400 $\left.\mathrm{MHz} \mathrm{CDCl}_{3}\right) \delta 8.14-8.11$ $(\mathrm{m}, 1 \mathrm{H}), 8.00-7.96(\mathrm{~m}, 1 \mathrm{H}), 7.88(\mathrm{~d}, J=8.0 \mathrm{~Hz}, 1 \mathrm{H}), 7.23(\mathrm{~d}, J=8.0 \mathrm{~Hz}$, 2H), $7.16(\mathrm{~d}, J=6.4 \mathrm{~Hz}, 1 \mathrm{H}), 7.08-6.99(\mathrm{~m}, 3 \mathrm{H}), 6.91(\mathrm{~d}, J=8.0 \mathrm{~Hz}$, $2 \mathrm{H}), 4.85(\mathrm{~s}, 1 \mathrm{H}), 4.71(\mathrm{~s}, 1 \mathrm{H}), 3.97(\mathrm{~s}, 3 \mathrm{H}), 3.20(\mathrm{~d}, J=8.0 \mathrm{~Hz}, 1 \mathrm{H}), 3.00$ $\left(\mathrm{dd}, J_{1}=15.2 \mathrm{~Hz}, J_{2}=3.2 \mathrm{~Hz}, 1 \mathrm{H}\right), 2.83\left(\mathrm{dd}, J_{1}=15.2 \mathrm{~Hz}, J_{2}=8.0 \mathrm{~Hz}, 1 \mathrm{H}\right), 2.49\left(\mathrm{td}, J_{1}=8.0 \mathrm{~Hz}, J_{2}=\right.$ $3.2 \mathrm{~Hz}, 1 \mathrm{H}), 2.24(\mathrm{~s}, 3 \mathrm{H}) .{ }^{13} \mathrm{C} \mathrm{NMR}\left(100 \mathrm{MHz}, \mathrm{CDCl}_{3}\right) \delta 196.2,166.4,143.4,143.1,142.4,141.6$, 137.7, 134.8, 134.3, 130.6, 129.1, 128.1, 127.8, 127.5, 126.1, 120.6, 120.5, 72.3, 70.3, 52.7, 44.1, 42.1, 
38.8, 21.5. HRMS (ESI-TOF) (m/z): Calcd for $\mathrm{C}_{27} \mathrm{H}_{23} \mathrm{NNaO}_{5} \mathrm{~S}$, $\left([\mathrm{M}+\mathrm{Na}]^{+}\right), 496.1189$, found 496.1189 .

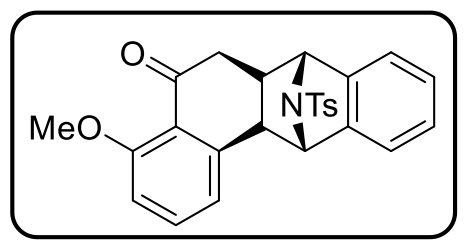

(rac)-4-methoxy-13-tosyl-6a,7,12,12a-tetrahydro-7,12-epiminote traphen-5(6H)-one (5fa)

Yellow solid (36 mg, 40\%), ${ }^{1} \mathrm{H} \mathrm{NMR}\left(400 \mathrm{MHz}, \mathrm{CDCl}_{3}\right) \delta 7.46(\mathrm{t}$, $J=8.0 \mathrm{~Hz}, 1 \mathrm{H}), 7.29(\mathrm{~d}, J=8.0 \mathrm{~Hz}, 2 \mathrm{H}), 7.12-7.08(\mathrm{~m}, 1 \mathrm{H}), 7.04$ - $6.96(\mathrm{~m}, 4 \mathrm{H}), 6.89\left(\mathrm{dd}, J_{1}=14.4 \mathrm{~Hz}, J_{2}=8.0 \mathrm{~Hz}, 3 \mathrm{H}\right), 4.86(\mathrm{~s}$, $1 \mathrm{H}), 4.79$ (s, 1H), 3.90 (s, 3H), 3.07 (d, J=8.4 Hz, 1H), $2.94\left(\mathrm{dd}, J_{1}=14.0 \mathrm{~Hz}, J_{2}=4.8 \mathrm{~Hz}, 1 \mathrm{H}\right), 2.82$ $\left(\mathrm{dd}, J_{1}=14.0 \mathrm{~Hz}, J_{2}=8.0 \mathrm{~Hz}, 1 \mathrm{H}\right), 2.41\left(\mathrm{td}, J_{1}=8.0 \mathrm{~Hz}, J_{2}=4.8 \mathrm{~Hz}, 1 \mathrm{H}\right), 2.24(\mathrm{~s}, 3 \mathrm{H}) .{ }^{13} \mathrm{C} \mathrm{NMR}(100$ $\left.\mathrm{MHz}, \mathrm{CDCl}_{3}\right) \delta 195.6,157.7,143.5,143.11,143.08,142.9,135.0,133.7,129.0,127.3,127.2,124.6$, 121.0, 120.5, 120.3, 110.4, 72.0, 69.8, 56.3, 44.7, 44.5, 39.0, 21.5. HRMS (ESI-TOF) (m/z): Calcd for $\mathrm{C}_{26} \mathrm{H}_{23} \mathrm{NNaO}_{4} \mathrm{~S},\left([\mathrm{M}+\mathrm{Na}]^{+}\right), 468.1240$, found 468.1235 .

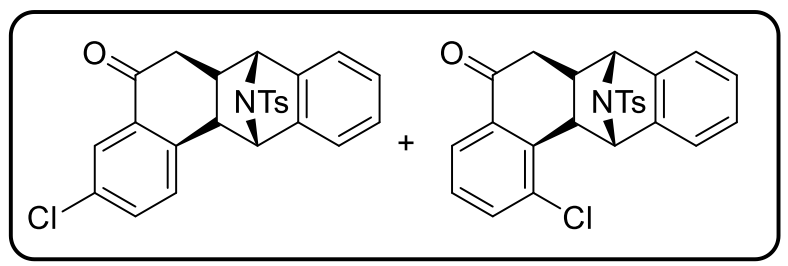

( rac)-3-chloro-13-tosyl-6a,7,12,12a-tetrahydro-7,12-epiminotetraphen-5(6H)-one (5ga) (rac)-1-chloro-13-tosyl-6a,7,12,12a-tetrahydro-7,12-epiminotetraphen-5(6H)-one (5ga')

(5ga:5ga' = 3.2:1)

Yellow solid (57.9 mg, 64\%), ${ }^{1} \mathrm{H}$ NMR (400 MHz, $\left.\mathrm{CDCl}_{3}\right) \delta 7.78\left(\mathrm{dd}, J_{1}=7.6 \mathrm{~Hz}, J_{2}=1.2 \mathrm{~Hz}\right.$, $1 \mathrm{H}), 7.76(\mathrm{~d}, J=2.0 \mathrm{~Hz}, 0.31 \mathrm{H}), 7.61\left(\mathrm{dd}, J_{1}=8.0 \mathrm{~Hz}, J_{2}=1.2 \mathrm{~Hz}, 1 \mathrm{H}\right), 7.48\left(\mathrm{dd}, J_{1}=8.0 \mathrm{~Hz}, J_{2}=2.0\right.$ $\mathrm{Hz}, 0.31 \mathrm{H}), 7.36(\mathrm{~d}, J=8.4 \mathrm{~Hz}, 0.31 \mathrm{H}), 7.32(\mathrm{t}, J=8.0 \mathrm{~Hz}, 1.0 \mathrm{H}), 7.27-7.26(\mathrm{~m}, 0.31 \mathrm{H}), 7.26-7.25$ (m, 1H), $7.25-7.23(\mathrm{~m}, 1.31 \mathrm{H}), 7.20-7.15(\mathrm{~m}, 1.31 \mathrm{H}), 7.09-7.00(\mathrm{~m}, 3.93 \mathrm{H}), 6.96(\mathrm{~s}, 0.31 \mathrm{H}), 6.95$ $-6.90(\mathrm{~m}, 2.31 \mathrm{H}), 4.87-4.84(\mathrm{~m}, 1.31 \mathrm{H}), 4.69(\mathrm{~s}, 1 \mathrm{H}), 4.62(\mathrm{~s}, 0.31 \mathrm{H}), 3.47(\mathrm{~d}, J=8.4 \mathrm{~Hz}, 1 \mathrm{H}), 3.12$ $(\mathrm{d}, J=8.0 \mathrm{~Hz}, 0.31 \mathrm{H}), 3.03-2.93(\mathrm{~m}, 1.31 \mathrm{H}), 2.83-2.75(\mathrm{~m}, 1.31 \mathrm{H}), 2.50-2.44(\mathrm{~m}, 1.31 \mathrm{H}), 2.28(\mathrm{~s}$, $0.93 \mathrm{H}), 2.25(\mathrm{~s}, 3 \mathrm{H}) .{ }^{13} \mathrm{C} \mathrm{NMR}\left(100 \mathrm{MHz}, \mathrm{CDCl}_{3}\right) \delta 195.6,195.4,143.5,143.31,143.25,142.4,139.9$, $139.0,136.4,135.7,134.9,134.1,133.5,133.3,130.6,129.1,128.1,127.84,127.76,127.4,125.7$, 124.7, 120.6, 120.4, 72.3, 70.9, 70.60, 70.57, 70.21, 70.18, 43.5, 41.8, 41.6, 38.7, 38.5, 21.5. HRMS $\left(\right.$ ESI-TOF) (m/z): Calcd for $\mathrm{C}_{25} \mathrm{H}_{20} \mathrm{ClNNaO}_{3} \mathrm{~S},\left([\mathrm{M}+\mathrm{Na}]^{+}\right), 472.0745$, found 472.0741 .

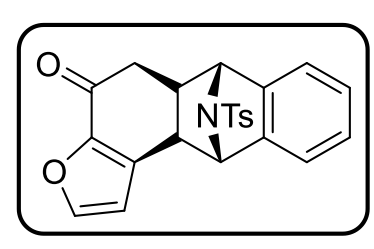

(rac)-12-tosyl-5a,6,11,11a-tetrahydro-6,11-epiminoanthra[2,1-b]furan -4(5H)-one (5ha)

Yellow solid (55.9 mg, 69\%), ${ }^{1} \mathrm{H}$ NMR $\left(400 \mathrm{MHz}, \mathrm{CDCl}_{3}\right) \delta 7.63(\mathrm{~d}, J=$ $2.0 \mathrm{~Hz}, 1 \mathrm{H}), 7.29-7.26(\mathrm{~m}, 2 \mathrm{H}), 7.09-7.06(\mathrm{~m}, 1 \mathrm{H}), 7.02-6.99(\mathrm{~m}$, $1 \mathrm{H}), 6.99-6.95(\mathrm{~m}, 2 \mathrm{H}), 6.93(\mathrm{~d}, J=8.4 \mathrm{~Hz}, 2 \mathrm{H}), 6.56(\mathrm{~d}, J=2.0 \mathrm{~Hz}$, $1 \mathrm{H}), 4.94(\mathrm{~s}, 1 \mathrm{H}), 4.75(\mathrm{~s}, 1 \mathrm{H}), 3.03-2.96(\mathrm{~m}, 2 \mathrm{H}), 2.90\left(\mathrm{dd}, J_{1}=17.6 \mathrm{~Hz}, J_{2}=5.6 \mathrm{~Hz}, 1 \mathrm{H}\right), 2.53(\mathrm{td}$, $\left.J_{1}=8.4 \mathrm{~Hz}, J_{2}=5.6 \mathrm{~Hz}, 1 \mathrm{H}\right), 2.25(\mathrm{~s}, 3 \mathrm{H}) .{ }^{13} \mathrm{C} \mathrm{NMR}\left(100 \mathrm{MHz}, \mathrm{CDCl}_{3}\right) \delta 183.4,148.2,147.0,143.4$, 142.8, 142.2, 136.5, 134.8, 129.1, 127.8, 127.34, 127.33, 120.7, 120.3, 111.1, 70.8, 69.3, 41.5, 40.4, 39.7, 21.5. HRMS (ESI-TOF) $(\mathrm{m} / \mathrm{z})$ : Calcd for $\mathrm{C}_{23} \mathrm{H}_{19} \mathrm{NNaO}_{4} \mathrm{~S}$, $\left([\mathrm{M}+\mathrm{Na}]^{+}\right), 428.0927$, found 428.0919 . 


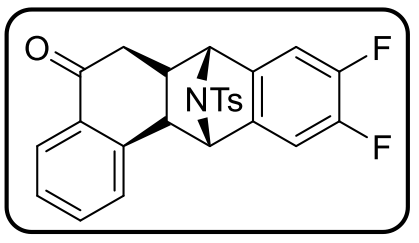

(rac)-9,10-difluoro-13-tosyl-6a,7,12,12a-tetrahydro-7,12-epiminotet raphen-5(6H)-one (5ab)

Yellow solid (60.8 mg, 67\%), ${ }^{1} \mathrm{H}$ NMR $\left(400 \mathrm{MHz}, \mathrm{CDCl}_{3}\right) \delta 7.82$ (dd, $\left.J_{1}=7.6 \mathrm{~Hz}, J_{2}=0.8 \mathrm{~Hz}, 1 \mathrm{H}\right), 7.55\left(\mathrm{td}, J_{1}=7.6 \mathrm{~Hz}, J_{2}=1.2 \mathrm{~Hz}, 1 \mathrm{H}\right)$, $7.41-7.33(\mathrm{~m}, 2 \mathrm{H}), 7.31-7.27(\mathrm{~m}, 2 \mathrm{H}), 7.05-6.99(\mathrm{~m}, 3 \mathrm{H}), 6.97-$ $6.91(\mathrm{~m}, 1 \mathrm{H}), 4.81(\mathrm{~s}, 1 \mathrm{H}), 4.67(\mathrm{~s}, 1 \mathrm{H}), 3.14(\mathrm{~d}, J=8.0 \mathrm{~Hz}, 1 \mathrm{H}), 2.92\left(\mathrm{dd}, J_{1}=15.6 \mathrm{~Hz}, J_{2}=3.6 \mathrm{~Hz}\right.$, $1 \mathrm{H}), 2.81\left(\mathrm{dd}, J_{1}=15.6 \mathrm{~Hz}, J_{2}=8.4 \mathrm{~Hz}, 1 \mathrm{H}\right), 2.46\left(\mathrm{td}, J_{1}=8.0 \mathrm{~Hz}, J_{2}=3.6 \mathrm{~Hz}, 1 \mathrm{H}\right), 2.31(\mathrm{~s}, 3 \mathrm{H}) .{ }^{13} \mathrm{C}$ NMR (100 MHz, $\left.\mathrm{CDCl}_{3}\right) \delta 196.3,150.8(J=15.2 \mathrm{~Hz}), 148.3(J=15.1 \mathrm{~Hz}), 144.0,140.8,139.6$, 139.0, 134.7 ( $J=18.0 \mathrm{~Hz}$ ), 133.7, 129.3, 129.0, 128.0, 127.4, 126.5, 126.0, 110.7, 110.6, 71.9, 69.9, 43.9, 41.8, 38.5, 21.5. HRMS (ESI-TOF) (m/z): Calcd for $\mathrm{C}_{25} \mathrm{H}_{19} \mathrm{~F}_{2} \mathrm{NNaO}_{3} \mathrm{~S},\left([\mathrm{M}+\mathrm{Na}]^{+}\right), 474.0946$, found 474.0934 .

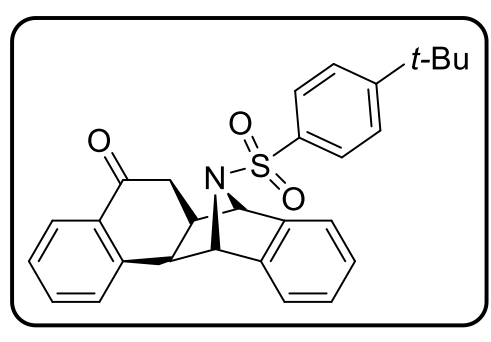

(rac)-13-((4-(tert-butyl)phenyl)sulfonyl)-6a,7,12,12a-tetrahydr o-7,12-epiminotetraphen-5(6H)-one (5ac)

Yellow solid (77.4 mg, 85\%), ${ }^{1} \mathrm{H}$ NMR (400 MHz, $\left.\mathrm{CDCl}_{3}\right) \delta 7.84$ $\left(\mathrm{dd}, J_{1}=7.6 \mathrm{~Hz}, J_{2}=1.2 \mathrm{~Hz}, 1 \mathrm{H}\right), 7.54\left(\mathrm{td}, J_{1}=7.6 \mathrm{~Hz}, J_{2}=1.2\right.$ $\mathrm{Hz}, 1 \mathrm{H}), 7.42$ (d, $J=7.6 \mathrm{~Hz}, 1 \mathrm{H}), 7.34$ (t, $J=7.6 \mathrm{~Hz}, 1 \mathrm{H}), 7.31-$ $7.26(\mathrm{~m}, 2 \mathrm{H}), 7.13-7.09(\mathrm{~m}, 3 \mathrm{H}), 7.05-7.02(\mathrm{~m}, 1 \mathrm{H}), 7.02-$ $6.94(\mathrm{~m}, 2 \mathrm{H}), 4.87(\mathrm{~s}, 1 \mathrm{H}), 4.71(\mathrm{~s}, 1 \mathrm{H}), 3.13(\mathrm{~d}, J=8.4 \mathrm{~Hz}, 1 \mathrm{H})$, $3.00\left(\mathrm{dd}, J_{1}=15.2 \mathrm{~Hz}, J_{2}=4.0 \mathrm{~Hz}, 1 \mathrm{H}\right), 2.85\left(\mathrm{dd}, J_{1}=15.6 \mathrm{~Hz}, J_{2}=8.4 \mathrm{~Hz}, 1 \mathrm{H}\right), 2.47\left(\mathrm{td}, J_{1}=8.4 \mathrm{~Hz}\right.$, $\left.J_{2}=4.0 \mathrm{~Hz}, 1 \mathrm{H}\right), 1.22(\mathrm{~s}, 9 \mathrm{H}) .{ }^{13} \mathrm{C} \mathrm{NMR}\left(100 \mathrm{MHz}, \mathrm{CDCl}_{3}\right) \delta 196.9,156.1,143.0,142.8,141.4,134.7$, 134.6, 133.5, 129.0, 127.7, 127.38, 127.35, 127.2, 126.0, 125.4, 120.5, 120.4, 72.3, 70.3, 44.1, 42.1, 38.6, 35.0, 31.1. HRMS (ESI-TOF) (m/z): Calcd for $\mathrm{C}_{28} \mathrm{H}_{27} \mathrm{NNaO}_{3} \mathrm{~S}$, $\left([\mathrm{M}+\mathrm{Na}]^{+}\right), 480.1604$, found 480.1595 .

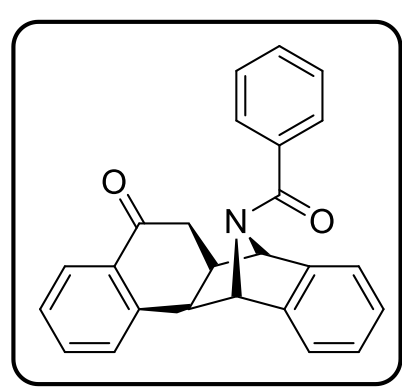

(rac)-13-benzoyl-6a,7,12,12a-tetrahydro-7,12-epiminotetraphen-5(6 H)-one (5ad)

Yellow solid (45.8 mg, 63\%), ${ }^{1} \mathrm{H}$ NMR (400 MHz, $\left.\mathrm{CDCl}_{3}\right) \delta 8.06-$ $7.81(\mathrm{br} \mathrm{s}, 1 \mathrm{H}), 7.63\left(\mathrm{td}, J_{1}=7.6 \mathrm{~Hz}, J_{2}=1.6 \mathrm{~Hz}, 1 \mathrm{H}\right), 7.57-7.27(\mathrm{~m}$, $6 \mathrm{H}), 7.25-7.16(\mathrm{~m}, 3 \mathrm{H}), 7.11-6.96(\mathrm{~m}, 1 \mathrm{H}), 6.82-6.48$ (br s, $1 \mathrm{H})$, $5.60-5.12(\mathrm{~m}, 1 \mathrm{H}), 5.12-4.64(\mathrm{~m}, 1 \mathrm{H}), 3.32(\mathrm{~d}, J=8.0 \mathrm{~Hz}, 1 \mathrm{H}), 3.09$ $-3.00(\mathrm{~m}, 1 \mathrm{H}), 2.92\left(\mathrm{dd}, J_{1}=15.6 \mathrm{~Hz}, J_{2}=8.8 \mathrm{~Hz}, 1 \mathrm{H}\right), 2.57\left(\mathrm{td}, J_{1}=\right.$ $\left.8.4 \mathrm{~Hz}, J_{2}=2.4 \mathrm{~Hz}, 1 \mathrm{H}\right) .{ }^{13} \mathrm{C} \mathrm{NMR}\left(100 \mathrm{MHz}, \mathrm{CDCl}_{3}\right) \delta 212.4,169.8$, 142.4, 134.7, 133.8, 130.8, 129.5, 128.3, 127.8, 127.5, 127.4, 126.0, 120.6, 120.0, 73.2, 66.1, 44.2, 42.0, 37.8. HRMS (ESI-TOF) (m/z): Calcd for $\mathrm{C}_{25} \mathrm{H}_{19} \mathrm{NNaO}_{2}$, $\left([\mathrm{M}+\mathrm{Na}]^{+}\right), 388.1308$, found 388.1299 .

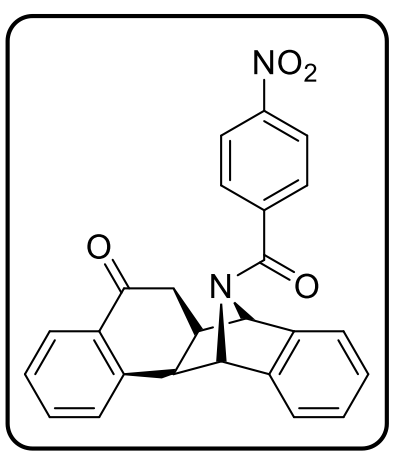

(rac)-13-(4-nitrobenzoyl)-6a,7,12,12a-tetrahydro-7,12-epiminotetrap hen-5(6H)-one (5ae)

Yellow solid (43.7 mg, 53\%), ${ }^{1} \mathrm{H} \mathrm{NMR}\left(400 \mathrm{MHz}, \mathrm{CDCl}_{3}\right) \delta 8.33-7.80$ $(\mathrm{m}, 3 \mathrm{H}), 7.65\left(\mathrm{td}, J_{1}=7.6 \mathrm{~Hz}, J_{2}=1.2 \mathrm{~Hz}, 1 \mathrm{H}\right), 7.59-7.36(\mathrm{br} \mathrm{s}, 4 \mathrm{H})$, $7.35-7.26(\mathrm{~m}, 1 \mathrm{H}), 7.25-7.08(\mathrm{~m}, 2 \mathrm{H}), 6.85-6.68$ (br s, 1H), $5.58-$ $5.16(\mathrm{~m}, 1 \mathrm{H}), 4.96-4.53(\mathrm{~m}, 1 \mathrm{H}), 3.37(\mathrm{~d}, J=8.0 \mathrm{~Hz}, 1 \mathrm{H}), 3.12-2.88$ $(\mathrm{m}, 2 \mathrm{H}), 2.66-2.57(\mathrm{~m}, 1 \mathrm{H}) .{ }^{13} \mathrm{C} \mathrm{NMR}\left(150 \mathrm{MHz}, \mathrm{CDCl}_{3}\right) \delta 196.2$, $166.9,142.1,140.3,134.8,134.0,129.5,128.7,127.8,127.6,126.0$, 
123.5, 120.7, 120.0, 73.2, 66.3, 41.8, 29.4, 27.3. HRMS (ESI-TOF) (m/z): Calcd for $\mathrm{C}_{25} \mathrm{H}_{18} \mathrm{~N}_{2} \mathrm{NaO}_{4}$, $\left([\mathrm{M}+\mathrm{Na}]^{+}\right), 433.1159$, found 433.1151 .

General procedure for preparation of 6.

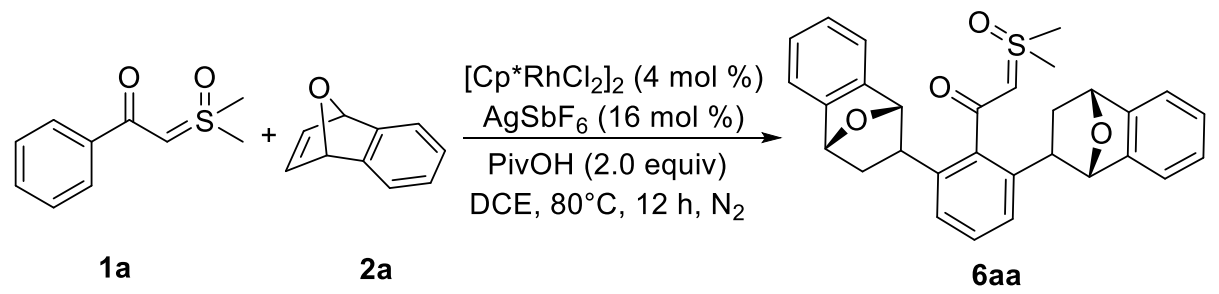

Representative Synthesis of Product 6: A pressure tube was charged with 1a $(39.2 \mathrm{mg}, 0.2 \mathrm{mmol}), 2 \mathbf{a}$ (72.0 mg, $0.5 \mathrm{mmol}$ ), [Cp* $\left.\mathrm{RhCl}_{2}\right]_{2}$ (4.9 mg, $4 \mathrm{~mol} \%$ ), AgSbF 6 (11.0 mg, $16 \mathrm{~mol} \%$ ), PivOH (40.8 mg, $0.4 \mathrm{mmol})$ and anhydrous DCE $(2.0 \mathrm{~mL})$. The reaction mixture was stirred at $80{ }^{\circ} \mathrm{C}$ for $12 \mathrm{~h}$ under $\mathrm{N}_{2}$. After the reaction was completed as indicated by TLC analysis, the solvent was removed under reduced pressure and the residue was purified by silica gel chromatography using petroleum ether/ethyl acetate $1: 1(\mathrm{v} / \mathrm{v})$ to give the corresponding product $\mathbf{6 a a}(88.8 \mathrm{mg}, 92 \%)$.
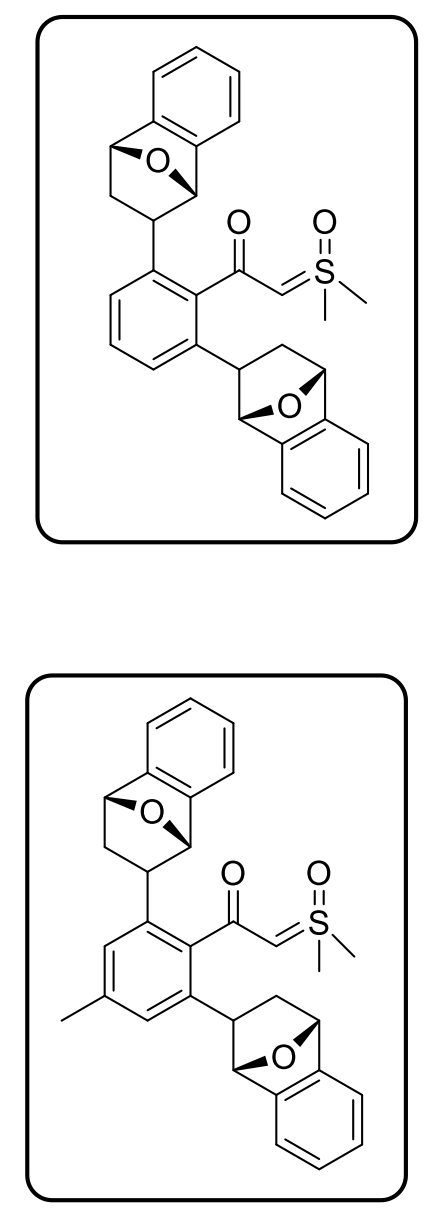

(rac)-1-(2,6-bis(1,2,3,4-tetrahydro-1,4-epoxynaphthalen-2-yl)phenyl )-2-(dimethyl(oxo)- $\lambda 6$-sulfanylidene)ethan-1-one (6aa)

White solid (88.8 mg, 92\%), ${ }^{1} \mathrm{H}$ NMR $\left(400 \mathrm{MHz}, \mathrm{CDCl}_{3}\right) \delta 7.48$ (d, $J=$ $7.6 \mathrm{~Hz}, 2 \mathrm{H}), 7.35(\mathrm{t}, J=8.0 \mathrm{~Hz}, 1 \mathrm{H}), 7.30-7.23(\mathrm{~m}, 4 \mathrm{H}), 7.19-7.07$ (br s, 4H), 5.55 (d, $J=4.4 \mathrm{~Hz}, 2 \mathrm{H}), 5.31-5.12(\mathrm{~m}, 2 \mathrm{H}), 4.68-4.26(\mathrm{~m}$, $1 \mathrm{H}), 3.23-3.14(\mathrm{~m}, 6 \mathrm{H}), 3.13-3.06(\mathrm{br} \mathrm{s}, 2 \mathrm{H}), 2.26-2.13(\mathrm{~m}, 2 \mathrm{H})$, $2.02-1.94(\mathrm{~m}, 2 \mathrm{H}) .{ }^{13} \mathrm{C}$ NMR $\left(100 \mathrm{MHz}, \mathrm{CDCl}_{3}\right) \delta 187.1,146.34$, $146.30,146.0,141.9,140.2,129.0,126.8,126.5,124.8,124.6,119.5$, 119.2, 118.7, 118.2, 85.7, 79.3, 79.2, 72.8, 42.4, 42.0, 41.8, 38.0, 37.6. HRMS (ESI-TOF) (m/z): Calcd for $\mathrm{C}_{30} \mathrm{H}_{28} \mathrm{NaO}_{4} \mathrm{~S}$, $\left([\mathrm{M}+\mathrm{Na}]^{+}\right)$, 507.1601, found 507.1597.

(rac)-2-(dimethyl(oxo)- $\lambda^{6}$-sulfanylidene)-1-(4-methyl-2,6-bis(1,2,3,4-t etrahydro-1,4-epoxynaphthalen-2-yl)phenyl)ethan-1-one (6ba) White solid (82.6 mg, 83\%), ${ }^{1} \mathrm{H}$ NMR $\left(400 \mathrm{MHz}, \mathrm{CDCl}_{3}\right) \delta 7.31-7.26$ (m, 5H), $7.25-7.22(\mathrm{~m}, 1 \mathrm{H}), 7.18-7.08(\mathrm{~m}, 4 \mathrm{H}), 5.56(\mathrm{~d}, J=4.4 \mathrm{~Hz}$, 2H), $5.29-5.14(\mathrm{~m}, 2 \mathrm{H}), 4.64-4.25(\mathrm{~m}, 1 \mathrm{H}), 3.22-3.17(\mathrm{~m}, 6 \mathrm{H}), 3.11$ $-3.03(\mathrm{~m}, 2 \mathrm{H}), 2.34$ (s, 3H), $2.25-2.13(\mathrm{~m}, 2 \mathrm{H}), 2.01-1.92(\mathrm{~m}, 2 \mathrm{H})$. ${ }^{13} \mathrm{C}$ NMR $\left(100 \mathrm{MHz}, \mathrm{CDCl}_{3}\right) \delta 187.6,146.6,146.5,146.1,140.2,139.5$, 138.8, 126.8, 126.7, 126.6, 125.42, 125.35, 125.3, 119.6, 119.3, 118.8, 118.3, 85.8, 85.7, 79.4, 72.7, 42.4, 42.2, 42.1, 38.1, 37.7, 21.9. HRMS (ESI-TOF) $(\mathrm{m} / \mathrm{z})$ : Calcd for $\mathrm{C}_{31} \mathrm{H}_{30} \mathrm{NaO}_{4} \mathrm{~S}$, $\left([\mathrm{M}+\mathrm{Na}]^{+}\right), 521.1757$, found 521.1755 . 


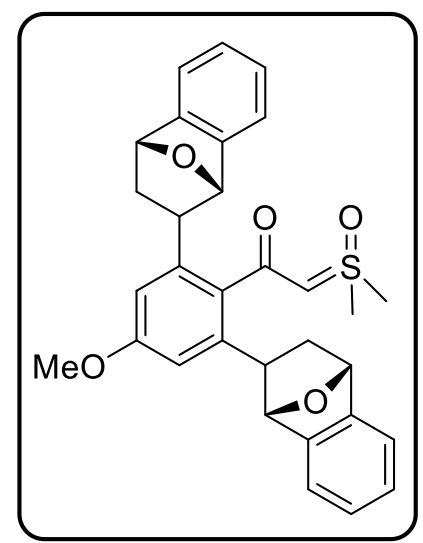

(rac)-2-(dimethyl(oxo)- $\lambda^{6}$-sulfanylidene)-1-(4-methoxy-2,6-bis(1,2, 3,4-tetrahydro-1,4-epoxynaphthalen-2-yl)phenyl)ethan-1-one (6ca)

Colorless liquid (91.4 mg, 89\%), ${ }^{1} \mathrm{H}$ NMR (400 MHz, $\left.\mathrm{CDCl}_{3}\right) \delta 7.29$ - 7.26 (m, 2H), $7.26-7.22(\mathrm{~m}, 2 \mathrm{H}), 7.17-7.10(\mathrm{~m}, 4 \mathrm{H}), 7.04$ (s, 2H), $5.54(\mathrm{~d}, J=4.8 \mathrm{~Hz}, 2 \mathrm{H}), 5.33-5.11($ br s, $2 \mathrm{H}), 4.65-4.23(\mathrm{~m}, 1 \mathrm{H})$, $3.85(\mathrm{~s}, 3 \mathrm{H}), 3.20-3.15(\mathrm{~m}, 6 \mathrm{H}), 3.10-3.05(\mathrm{~m}, 2 \mathrm{H}), 2.21-2.13(\mathrm{~m}$, $2 \mathrm{H}), 2.01-1.93(\mathrm{~m}, 2 \mathrm{H}) .{ }^{13} \mathrm{C} \mathrm{NMR}\left(100 \mathrm{MHz}, \mathrm{CDCl}_{3}\right) \delta 187.4,160.1$, $146.42,146.39,146.1,146.0,142.2$, 135.1, 126.8, 126.6, 119.43, $119.35,118.8,118.4,110.2,85.7,85.6,79.3,73.0,55.5,42.6,42.5$, 42.1, 41.9, 38.1, 38.0, 29.4, 27.3. HRMS (ESI-TOF) (m/z): Calcd for $\mathrm{C}_{31} \mathrm{H}_{30} \mathrm{NaO}_{5} \mathrm{~S},\left([\mathrm{M}+\mathrm{Na}]^{+}\right), 537.1706$, found 537.1704 .
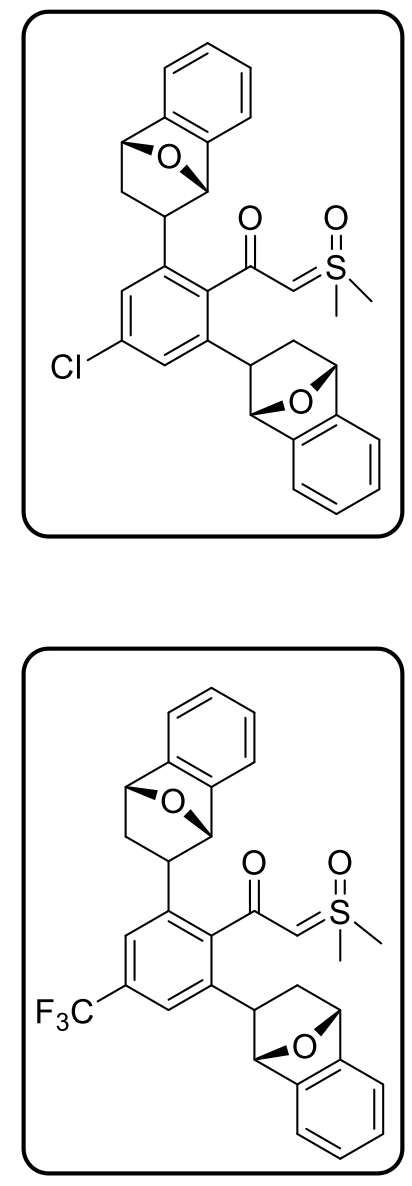

(rac)-1-(2,6-bis(1,2,3,4-tetrahydro-1,4-epoxynaphthalen-2-yl)-4-(trifl uoromethyl)phenyl)-2-(dimethyl(oxo)- $\lambda^{6}$-sulfanylidene)ethan-1-one (6ea)

White solid (92.9 mg, 84\%), ${ }^{1} \mathrm{H}$ NMR $\left(400 \mathrm{MHz}, \mathrm{CDCl}_{3}\right) \delta 7.74(\mathrm{~s}, 2 \mathrm{H})$, $7.32-7.26(\mathrm{~m}, 3 \mathrm{H}), 7.26-7.22(\mathrm{~m}, 1 \mathrm{H}), 7.21-7.10(\mathrm{~m}, 4 \mathrm{H}), 5.58(\mathrm{~d}, J$ $=3.6 \mathrm{~Hz}, 2 \mathrm{H}), 5.25(\mathrm{~d}, J=1.6 \mathrm{~Hz}, 1 \mathrm{H}), 5.18(\mathrm{~d}, J=11.6 \mathrm{~Hz}, 1 \mathrm{H}), 4.67-$ $4.28(\mathrm{~m}, 1 \mathrm{H}), 3.22(\mathrm{~s}, 3 \mathrm{H}), 3.20(\mathrm{~s}, 3 \mathrm{H}), 3.16-3.09$ (br s, 2H), $2.23-$ $2.12(\mathrm{~m}, 2 \mathrm{H}), 2.06-1.94(\mathrm{~m}, 2 \mathrm{H}) .{ }^{13} \mathrm{C} \mathrm{NMR}\left(100 \mathrm{MHz}, \mathrm{CDCl}_{3}\right) \delta$ $185.7,146.10,146.06,144.9,141.7,131.2$ ( $J=31.7 \mathrm{~Hz}), 127.1,126.9$, $126.7,124.3$ ( $J=271.2 \mathrm{~Hz}), 121.9,121.7,119.7,119.4,118.9,118.3$, 85.6, 85.5, 85.4, 79.4, 79.2, 73.1, 42.5, 42.2, 42.0, 38.4, 38.0. HRMS (ESI-TOF) (m/z): Calcd for $\mathrm{C}_{31} \mathrm{H}_{27} \mathrm{~F}_{3} \mathrm{NaO}_{4} \mathrm{~S},\left([\mathrm{M}+\mathrm{Na}]^{+}\right), 575.1474$, found 575.1476 . 


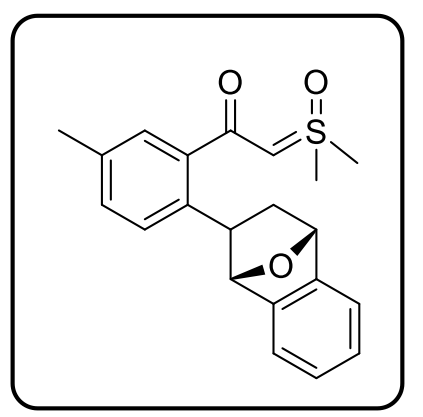

(rac)-2-(dimethyl(oxo)- $\lambda^{6}$-sulfanylidene)-1-(5-methyl-2-(-1,2,3,4-tetr ahydro-1,4-epoxynaphthalen-2-yl)phenyl)ethan-1-one (6fa)

Yellow liquid (44.6 mg, 63\%), ${ }^{1} \mathrm{H}$ NMR $\left(400 \mathrm{MHz}, \mathrm{CDCl}_{3}\right) \delta 7.57-$ $7.53(\mathrm{~m}, 1 \mathrm{H}), 7.31-7.29(\mathrm{~m}, 1 \mathrm{H}), 7.27-7.25(\mathrm{~m}, 1 \mathrm{H}), 7.20-7.17(\mathrm{~m}$, 2H), $7.16-7.12(\mathrm{~m}, 2 \mathrm{H}), 5.52(\mathrm{~d}, J=4.8 \mathrm{~Hz}, 1 \mathrm{H}), 5.25$ (br s, $1 \mathrm{H}), 4.69$ - $4.56(\mathrm{~m}, 1 \mathrm{H}), 3.44-3.37(\mathrm{~m}, 6 \mathrm{H}), 3.37-3.33(\mathrm{~m}, 1 \mathrm{H}), 2.33(\mathrm{~s}, 3 \mathrm{H})$, $2.15-2.09(\mathrm{~m}, 1 \mathrm{H}), 2.04-1.99(\mathrm{~m}, 1 \mathrm{H}) .{ }^{13} \mathrm{C}$ NMR $\left(100 \mathrm{MHz}, \mathrm{CDCl}_{3}\right)$ $\delta 187.4,146.4,146.3,141.4,139.3,135.4,130.4,127.6,127.2,126.6$, 119.2, 119.0, 85.7, 79.3, 71.8, 42.3, 42.2, 41.3, 38.2, 21.0. HRMS (ESI-TOF) (m/z): Calcd for $\mathrm{C}_{21} \mathrm{H}_{23} \mathrm{O}_{3} \mathrm{~S},\left([\mathrm{M}+\mathrm{H}]^{+}\right), 355.1362$, found 355.1364 .

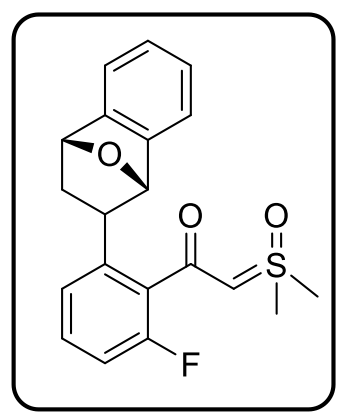

(rac)-2-(dimethyl(oxo)- $\lambda^{6}$-sulfanylidene) -1-(2-fluoro-6-(1,2,3,4 -tetrahydro-1,4-epoxynaphthalen-2-yl)phenyl)ethan-1-one (6ga) Colorless liquid (50.9 mg, 71\%), ${ }^{1} \mathrm{H}$ NMR $\left(400 \mathrm{MHz}, \mathrm{CDCl}_{3}\right) \delta 7.39$ (dd, $J_{1}$ $\left.=8.0 \mathrm{~Hz}, J_{2}=0.8 \mathrm{~Hz}, 1 \mathrm{H}\right), 7.32-7.26(\mathrm{~m}, 3 \mathrm{H}), 7.19-7.12(\mathrm{~m}, 2 \mathrm{H}), 6.95-$ $6.90(\mathrm{~m}, 1 \mathrm{H}), 5.54(\mathrm{~d}, J=4.8 \mathrm{~Hz}, 1 \mathrm{H}), 5.25(\mathrm{~s}, 1 \mathrm{H}), 4.61(\mathrm{~s}, 1 \mathrm{H}), 3.42(\mathrm{~s}$, $6 \mathrm{H}), 3.17(\mathrm{q}, J=4.4 \mathrm{~Hz}, 1 \mathrm{H}), 2.19-2.12(\mathrm{~m}, 1 \mathrm{H}), 2.04-1.98(\mathrm{~m}, 1 \mathrm{H}) .{ }^{13} \mathrm{C}$ NMR $\left(100 \mathrm{MHz}, \mathrm{CDCl}_{3}\right) \delta 181.2,158.7(J=243.5 \mathrm{~Hz}), 146.1(J=5.9$ $\mathrm{Hz}), 144.4(J=2.7 \mathrm{~Hz}), 130.1(J=18.5 \mathrm{~Hz}), 129.9(J=7.0 \mathrm{~Hz}), 126.9$, $126.7,122.5,119.3,119.0,113.4(J=23.4 \mathrm{~Hz}), 85.5,79.4,73.8,42.4,42.3,41.9$, 38.0. HRMS (ESI-TOF) (m/z): Calcd for $\mathrm{C}_{20} \mathrm{H}_{19} \mathrm{FNaO}_{3} \mathrm{~S}$, ([M + Na $\left.]^{+}\right), 381.0931$, found 381.0936.

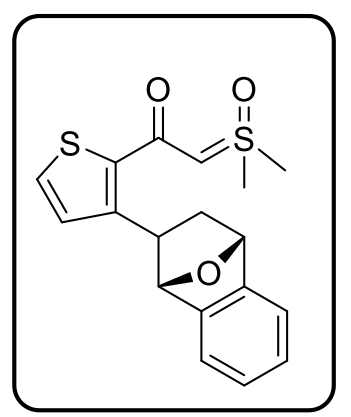

(rac)-2-(dimethyl(oxo)- $\lambda^{6}$-sulfanylidene)-1-(3-1,2,3,4-tetrahydro -1,4-epoxynaphthalen-2-yl)thiophen-2-yl)ethan-1-one (6ha)

White solid (52.3 mg, 76\%), ${ }^{1} \mathrm{H}$ NMR (400 MHz, $\left.\mathrm{CDCl}_{3}\right) \delta 7.32-7.28(\mathrm{~m}$, $2 \mathrm{H}), 7.27-7.26(\mathrm{~m}, 1 \mathrm{H}), 7.25-7.23(\mathrm{~m}, 1 \mathrm{H}), 7.18-7.13(\mathrm{~m}, 2 \mathrm{H}), 5.50(\mathrm{~d}$, $J=4.8 \mathrm{~Hz}, 1 \mathrm{H}), 5.27(\mathrm{~s}, 1 \mathrm{H}), 4.77$ (s, $1 \mathrm{H}), 3.91(\mathrm{q}, J=4.4 \mathrm{~Hz}, 1 \mathrm{H}), 3.45(\mathrm{~s}$, $3 \mathrm{H}), 3.43(\mathrm{~s}, 3 \mathrm{H}), 2.11-2.05(\mathrm{~m}, 1 \mathrm{H}), 2.02-1.96(\mathrm{~m}, 1 \mathrm{H}) .{ }^{13} \mathrm{C}$ NMR $(100$ $\left.\mathrm{MHz}, \mathrm{CDCl}_{3}\right) \delta 177.7,147.4,146.2,145.8,136.9,129.0,126.8,126.77$, $126.73,119.4,119.2,84.9,79.4,70.4,42.7,42.5,39.3,37.6$. HRMS (ESI-TOF) (m/z): Calcd for $\mathrm{C}_{18} \mathrm{H}_{18} \mathrm{NaO}_{3} \mathrm{~S}_{2}$, ([M+Na $\left.]^{+}\right), 369.0590$, found 369.0592 .

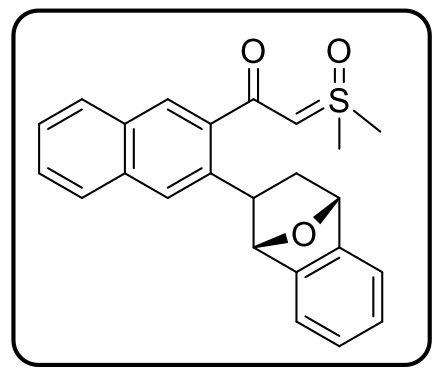

(rac)-2-(dimethyl(oxo)- $\lambda^{6}$-sulfanylidene)-1-(3-(1,2,3,4-tetrahydro1,4-epoxynaphthalen-2-yl)naphthalen-2-yl)ethan-1-one (6ia)

White solid (62.5 mg, 80\%), ${ }^{1} \mathrm{H}$ NMR $\left(400 \mathrm{MHz}, \mathrm{CDCl}_{3}\right) \delta 8.07$ (s, $1 \mathrm{H}), 7.89(\mathrm{~s}, 1 \mathrm{H}), 7.84(\mathrm{~d}, J=8.0 \mathrm{~Hz}, 1 \mathrm{H}), 7.80(\mathrm{~d}, J=8.0 \mathrm{~Hz}, 1 \mathrm{H})$, $7.51-7.42(\mathrm{~m}, 2 \mathrm{H}), 7.37-7.33(\mathrm{~m}, 1 \mathrm{H}), 7.31-7.28(\mathrm{~m}, 1 \mathrm{H}), 7.20-$ $7.14(\mathrm{~m}, 2 \mathrm{H}), 5.58(\mathrm{~d}, J=4.8 \mathrm{~Hz}, 1 \mathrm{H}), 5.41(\mathrm{~s}, 1 \mathrm{H}), 5.03-4.54(\mathrm{br} \mathrm{s}$, $1 \mathrm{H}), 3.53-3.42(\mathrm{~m}, 7 \mathrm{H}), 2.31-2.22(\mathrm{~m}, 1 \mathrm{H}), 2.12-2.06(\mathrm{~m}, 1 \mathrm{H})$. ${ }^{13} \mathrm{C}$ NMR $\left(100 \mathrm{MHz}, \mathrm{CDCl}_{3}\right) \delta 187.3,146.4,140.3,134.1,131.4,128.0,127.9,126.74,126.67,126.6$, 126.5, 125.97, 125.96, 119.3, 85.7, 79.5, 72.1, 42.5, 42.3, 41.9, 38.4. HRMS (ESI-TOF) (m/z): Calcd for $\mathrm{C}_{24} \mathrm{H}_{22} \mathrm{NaO}_{3} \mathrm{~S}$, $\left([\mathrm{M}+\mathrm{Na}]^{+}\right), 413.1182$, found 413.1183 . 


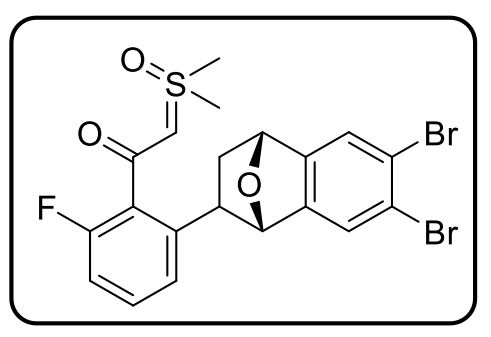

(rac)-1-(2-(6,7-dibromo-1,2,3,4-tetrahydro-1,4-epoxynaphthale n-2-yl)-6-fluorophenyl)-2-(dimethyl(oxo)- $\lambda^{6}$-sulfanylidene)etha n-1-one (6gb)

White solid (70.8 mg, 69\%), ${ }^{1} \mathrm{H}$ NMR (600 MHz, $\left.\mathrm{CDCl}_{3}\right) \delta 7.58$ (s, 1H), $7.53(\mathrm{~s}, 1 \mathrm{H}), 7.32-7.29(\mathrm{~m}, 1 \mathrm{H}), 7.29-7.26(\mathrm{~m}, 1 \mathrm{H})$, $6.94(\mathrm{t}, J=8.4 \mathrm{~Hz}, 1 \mathrm{H}), 5.49(\mathrm{~d}, J=4.8 \mathrm{~Hz}, 1 \mathrm{H}), 5.22(\mathrm{~s}, 1 \mathrm{H})$, $4.78-4.51$ (br s, $1 \mathrm{H}), 3.48(\mathrm{~s}, 3 \mathrm{H}), 3.46(\mathrm{~s}, 3 \mathrm{H}), 3.20-3.14(\mathrm{~m}$, 1H), $2.20-2.15(\mathrm{~m}, 1 \mathrm{H}), 2.01-1.97(\mathrm{~m}, 1 \mathrm{H}) .{ }^{13} \mathrm{C} \mathrm{NMR}\left(100 \mathrm{MHz}, \mathrm{CDCl}_{3}\right) \delta 180.9,158.8(J=243.9$ $\mathrm{Hz}), 147.2(J=7.1 \mathrm{~Hz}), 143.4,130.2,130.0(J=7.2 \mathrm{~Hz}), 124.7(J=29.5 \mathrm{~Hz}), 122.7,122.6,122.3$, $113.8(\quad J=22.6 \mathrm{~Hz}), 85.0,78.9,74.4,42.5,42.4,41.8,37.2$. HRMS (ESI-TOF) $(\mathrm{m} / \mathrm{z})$ : Calcd for $\mathrm{C}_{20} \mathrm{H}_{17} \mathrm{Br}_{2} \mathrm{FNaO}_{3} \mathrm{~S}$, $\left([\mathrm{M}+\mathrm{Na}]^{+}\right)$, 536.9141, found 536.9134 . 
III. Preparation of 3ba in Gram Scale

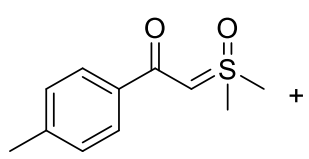

1b (4.76 mmol 1.0g)

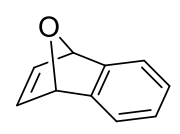

$2 \mathbf{a}$

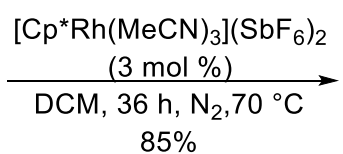

$85 \%$

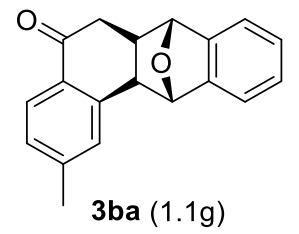

A pressure tube was charged with $\mathbf{1 b}(1.0 \mathrm{~g}, 4.76 \mathrm{mmol}), \mathbf{2 a}(1.4 \mathrm{~g}, 2.0$ equiv), $\left[\mathrm{Cp} * \mathrm{Rh}(\mathrm{MeCN})_{3}\right]\left(\mathrm{SbF}_{6}\right)_{2}(119.0 \mathrm{mg}, 3 \mathrm{~mol} \%)$, and anhydrous DCM $(25 \mathrm{~mL})$. The reaction mixture was stirred at $70{ }^{\circ} \mathrm{C}$ for $36 \mathrm{~h}$ under $\mathrm{N}_{2}$. After the reaction was completed as indicated by TLC analysis, the solvent was removed under reduced pressure and the residue was purified by silica gel chromatography using petroleum ether/ethyl acetate 15:1 (v/v) to give the corresponding product 3ba $(1.1 \mathrm{~g}, 85 \%)$. 


\section{Derivatization of $3 \mathrm{ba}$}

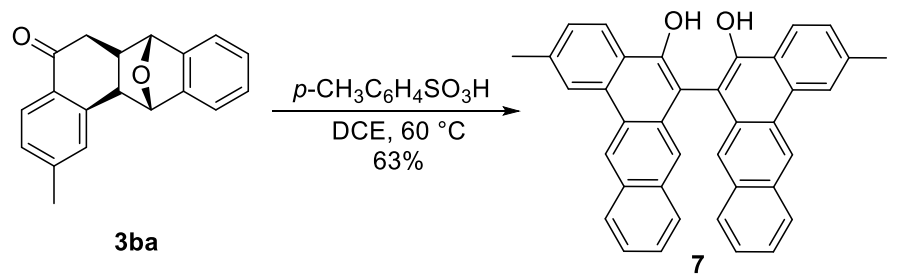

A pressure tube was charged with $3 \mathbf{b a}(55.2 \mathrm{mg}, 0.2 \mathrm{mmol}), p-\mathrm{CH}_{3} \mathrm{C}_{6} \mathrm{H}_{4} \mathrm{SO}_{3} \mathrm{H}(17.2 \mathrm{mg})$, and anhydrous DCE $(2 \mathrm{~mL})$. The reaction mixture was stirred at $60{ }^{\circ} \mathrm{C}$ for $36 \mathrm{~h}$. After the reaction was completed as indicated by TLC analysis, the solvent was removed under reduced pressure and the residue was purified by silica gel chromatography using petroleum ether/ethyl acetate 30:1 (v/v) to give the corresponding product 7 (32.3 mg, 63\%).

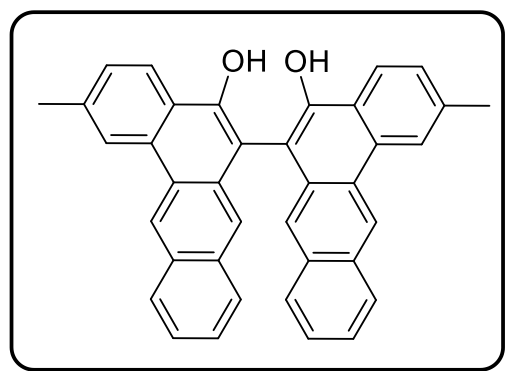

\section{2,2'-dimethyl-[6,6'-bitetraphene]-5,5'-diol (7)}

White solid (32.3 mg, $63 \%),{ }^{1} \mathrm{H}$ NMR $\left(400 \mathrm{MHz}, \mathrm{CDCl}_{3}\right) \delta$ $9.27(\mathrm{~s}, 2 \mathrm{H}), 8.80(\mathrm{~s}, 2 \mathrm{H}), 8.36(\mathrm{~d}, J=8.0 \mathrm{~Hz}, 2 \mathrm{H}), 8.11(\mathrm{~d}, J=$ $8.0 \mathrm{~Hz}, 2 \mathrm{H}), 7.78(\mathrm{~s}, 2 \mathrm{H}), 7.62-7.56(\mathrm{~m}, 4 \mathrm{H}), 7.47-7.43(\mathrm{~m}$, $2 \mathrm{H}), 7.37-7.32(\mathrm{~m}, 2 \mathrm{H}), 5.69$ (s, 2H), 2.77 (s, 6H). ${ }^{13} \mathrm{C} \mathrm{NMR}$ $\left(100 \mathrm{MHz}, \mathrm{CDCl}_{3}\right) \delta 149.8,138.7,132.7,132.5,130.8,130.4$, $129.0,128.3,127.8,126.6,126.0,125.3,123.9,123.6,123.2$, 123.0, 122.3, 105.7, 22.4. HRMS (ESI-TOF) (m/z): Calcd for $\mathrm{C}_{38} \mathrm{H}_{26} \mathrm{NaO}_{2},\left([\mathrm{M}+\mathrm{Na}]^{+}\right), 537.1825$, found 537.1836.

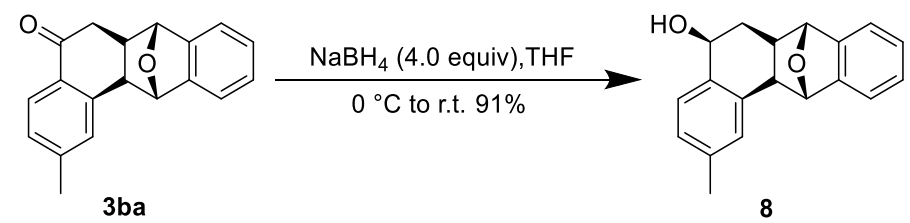

To a solution of $\mathbf{3 b a}(55.2 \mathrm{mg}, 0.2 \mathrm{mmol})$, in THF $(2 \mathrm{~mL})$ was added $\mathrm{NaBH}_{4}(30.3 \mathrm{mg}, 0.8 \mathrm{mmol})$ carefully at $0{ }^{\circ} \mathrm{C}$ under $\mathrm{N}_{2}$ and the mixture was stirred at the same temperature for $15 \mathrm{~min}$. Then the mixture was stirred at room temperature for $1 \mathrm{~h}$ before quenched with water. The mixture was extracted with ethylacetate, and the combined organic extracts were washed with water and brine, and dried over $\mathrm{Na}_{2} \mathrm{SO}_{4}$. The solvent was evaporated under the reduced pressure and the residue was purified by silica gel chromatography using PE/EA to afford compound 8 (50.4 mg, 91 \%).

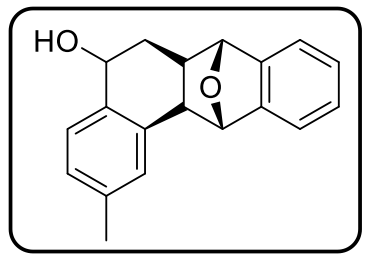

(rac)-2-methyl-5,6,6a,7,12,12a-hexahydro-7,12-epoxytetraphen-5-ol (8) White solid (50.4 mg, $91 \%),{ }^{1} \mathrm{H}$ NMR (400 MHz, $\left.\mathrm{CDCl}_{3}\right) \delta 7.41-7.38$ (m, 1H), $7.36-7.33(\mathrm{~m}, 1 \mathrm{H}), 7.30(\mathrm{~d}, J=7.6 \mathrm{~Hz}, 1 \mathrm{H}), 7.26-7.22(\mathrm{~m}$, $2 \mathrm{H}), 7.19-7.17(\mathrm{~m}, 1 \mathrm{H}), 7.10\left(\mathrm{dd}, J_{1}=8.0 \mathrm{~Hz}, J_{2}=1.6 \mathrm{~Hz}, 1 \mathrm{H}\right), 5.38(\mathrm{~s}$, $1 \mathrm{H}), 5.11(\mathrm{~s}, 1 \mathrm{H}), 4.69\left(\mathrm{dt}, J_{1}=11.2 \mathrm{~Hz}, J_{2}=3.2 \mathrm{~Hz}, 1 \mathrm{H}\right), 4.24(\mathrm{~d}, J=12.0$ $\mathrm{Hz}, 1 \mathrm{H}), 3.14(\mathrm{~d}, J=8.4 \mathrm{~Hz}, 1 \mathrm{H}), 2.49-2.44(\mathrm{~m}, 1 \mathrm{H}), 2.40(\mathrm{~s}, 3 \mathrm{H}), 2.40-2.36(\mathrm{~m}, 1 \mathrm{H}), 2.02\left(\mathrm{qd}, J_{1}=\right.$ $\left.7.6 \mathrm{~Hz}, J_{2}=3.6 \mathrm{~Hz}, 1 \mathrm{H}\right) .{ }^{13} \mathrm{C}$ NMR $\left(100 \mathrm{MHz}, \mathrm{CDCl}_{3}\right) \delta 145.3,144.2,138.4,137.2,136.7,130.4$, $129.5,127.9,127.3,127.2,119.5,119.3,87.2,86.3,67.7,44.4,37.9,34.1,21.4$. HRMS (ESI-TOF) $(\mathrm{m} / \mathrm{z})$ : Calcd for $\mathrm{C}_{19} \mathrm{H}_{18} \mathrm{NaO}_{2},\left([\mathrm{M}+\mathrm{Na}]^{+}\right), 301.1199$, found 301.1205 . 


\section{Mechanistic Studies}

\section{(1) H/D Exchange Experiments}

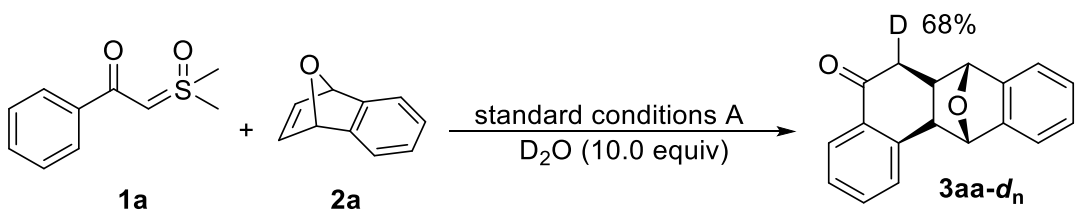

$72 \%$

A pressure tube was charged with 1 a $(39.2 \mathrm{mg}, 0.2 \mathrm{mmol}), 2 \mathrm{2a}(57.7 \mathrm{mg}, 0.4 \mathrm{mmol})$, $\left[\mathrm{Cp} * \mathrm{Rh}(\mathrm{MeCN})_{3}\right]\left(\mathrm{SbF}_{6}\right)_{2}(10.0 \mathrm{mg}, 6 \mathrm{~mol} \%), \mathrm{D}_{2} \mathrm{O}(36 \mathrm{uL}, 10.0$ equiv) and anhydrous DCM (2.0 mL). The reaction mixture was stirred at $70{ }^{\circ} \mathrm{C}$ for $36 \mathrm{~h}$ under $\mathrm{N}_{2}$. The solvent was removed under reduced pressure and the residue was purified by silica gel chromatography using petroleum ether/ethyl acetate 15:1 (v/v) to give the corresponding product 3aa $(37.6 \mathrm{mg}, 72 \%)$. and the extent of deuteration was obtained by ${ }^{1} \mathrm{H}$ NMR analysis.

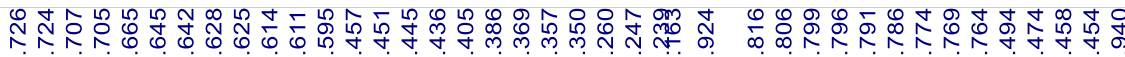

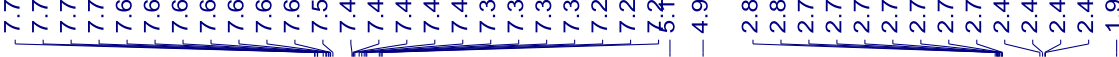

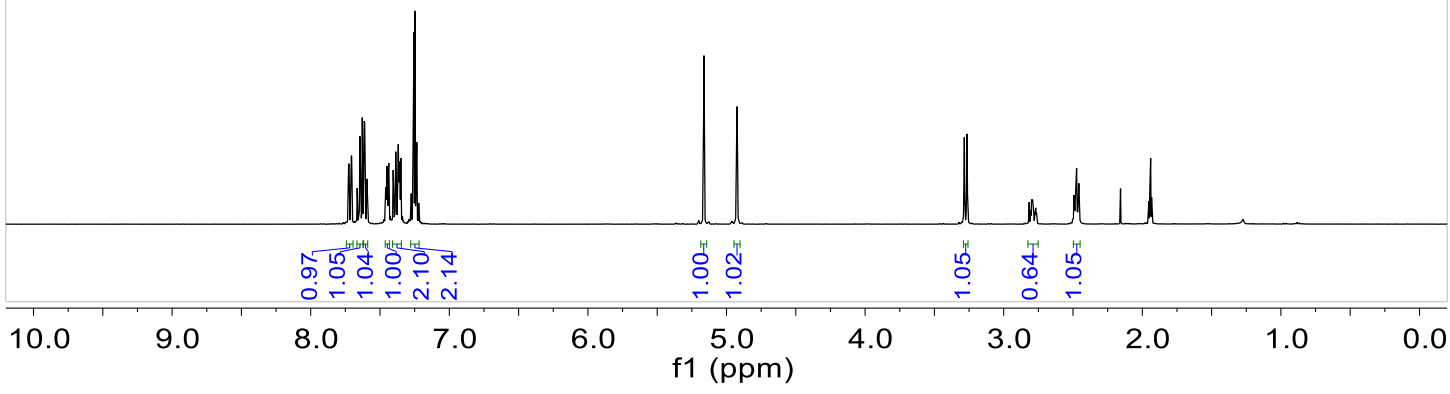

(2) Independent KIE studies:
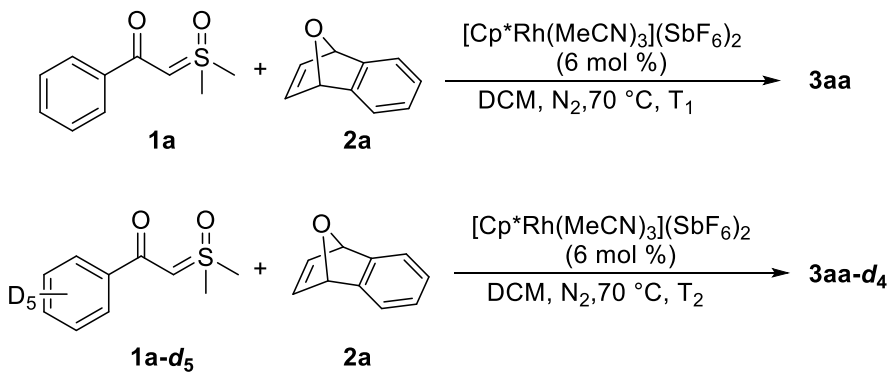
A pressure tube was charged with $\mathbf{1 a}(19.6 \mathrm{mg}, 0.1 \mathrm{mmol}), \mathbf{2 a}(28.8 \mathrm{mg}, 0.2 \mathrm{mmol})$, $\left[\mathrm{Cp} * \mathrm{Rh}(\mathrm{MeCN})_{3}\right]\left(\mathrm{SbF}_{6}\right)_{2}(5.0 \mathrm{mg}, 6 \mathrm{~mol} \%)$, and anhydrous DCM $(1.0 \mathrm{~mL})$. To another tube was added 1a-d5 $(20.1 \mathrm{mg}, 0.1 \mathrm{mmol}), \mathbf{2 a}(28.8 \mathrm{mg}, 0.2 \mathrm{mmol}),\left[\mathrm{Cp} * \mathrm{Rh}(\mathrm{MeCN})_{3}\right]\left(\mathrm{SbF}_{6}\right)_{2}(5.0 \mathrm{mg}, 6$ mol \%), and anhydrous DCM (1.0 mL). These two reaction mixtures were stirred side-by-side in the same oil bath at $70{ }^{\circ} \mathrm{C}$ under $\mathrm{N}_{2}$ for a certain time. Then, the reaction was quenched by flash column chromatrography, the filtrate was removed under reduced pressure and the residue was mixed with internal standard substance 1,3,5-trimethoxybenzene $(16.8 \mathrm{mg})$, KIE value $\left(k_{\mathrm{H}} / k_{\mathrm{D}}=3.9\right)$ was determined on the basis of ${ }^{1} \mathrm{H}$ NMR analysis.

\begin{tabular}{|l|l|l|l|}
\hline \multicolumn{2}{|l|}{ 3aa } & 3 aa- $d_{4}$ \\
\hline $\mathrm{T}_{1}(\mathrm{~min})$ & Yield $(\%)$ & $\mathrm{T}_{2}(\mathrm{~min})$ & Yield $(\%)$ \\
\hline 40 & 14 & 60 & 17 \\
\hline 60 & 23 & 90 & 20 \\
\hline 80 & 32 & 120 & 24 \\
\hline
\end{tabular}

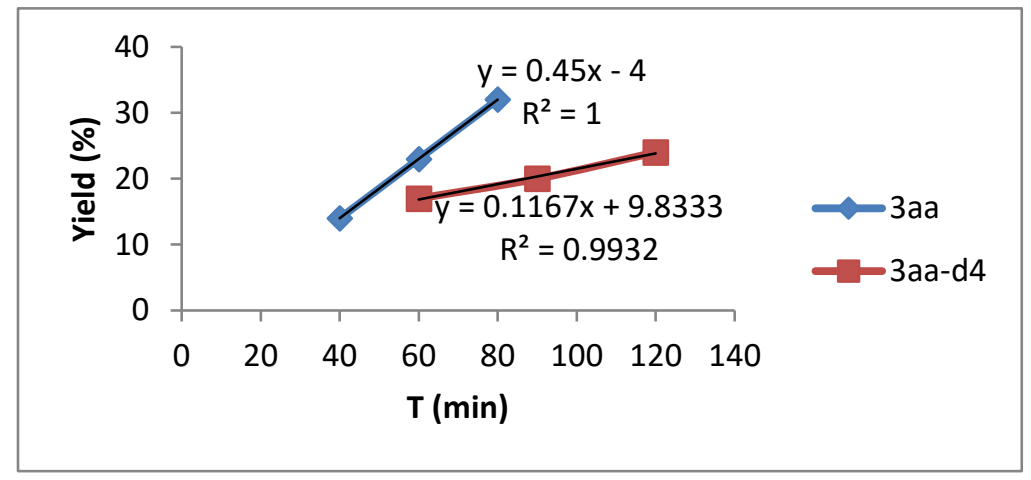

\section{(3) competition experiment}<smiles>COc1ccc(C(=O)C[SH](C)(C)=O)cc1</smiles>

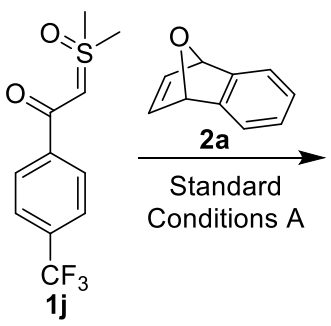<smiles>COc1ccc2c(c1)C1OC(CC1=O)C1OC2c2ccccc21</smiles>

A pressure tube was charged with $\mathbf{1 d}(22.6 \mathrm{mg}, 0.1 \mathrm{mmol}), \mathbf{1 j}$ (26.4 mg, $0.1 \mathrm{mmol}), \mathbf{2 a}(57.6 \mathrm{mg}$, $0.4 \mathrm{mmol}),\left[\mathrm{Cp} * \mathrm{Rh}(\mathrm{MeCN})_{3}\right]\left(\mathrm{SbF}_{6}\right)_{2}(10.0 \mathrm{mg}, 12 \mathrm{~mol} \%)$ and anhydrous DCM $(2.0 \mathrm{~mL})$. The reaction mixture was stirred at $70{ }^{\circ} \mathrm{C}$ for $36 \mathrm{~h}$ under $\mathrm{N}_{2}$. After the reaction was completed as indicated by TLC analysis, the solvent was removed under reduced pressure and the residue was purified by silica gel chromatography using petroleum ether/ethyl acetate 15:1 (v/v) to afford product 3da and 3ja. The ratio of 3da:3ja $=10: 1$ was determined on the basis of ${ }^{1} \mathrm{H}$ NMR analysis. 


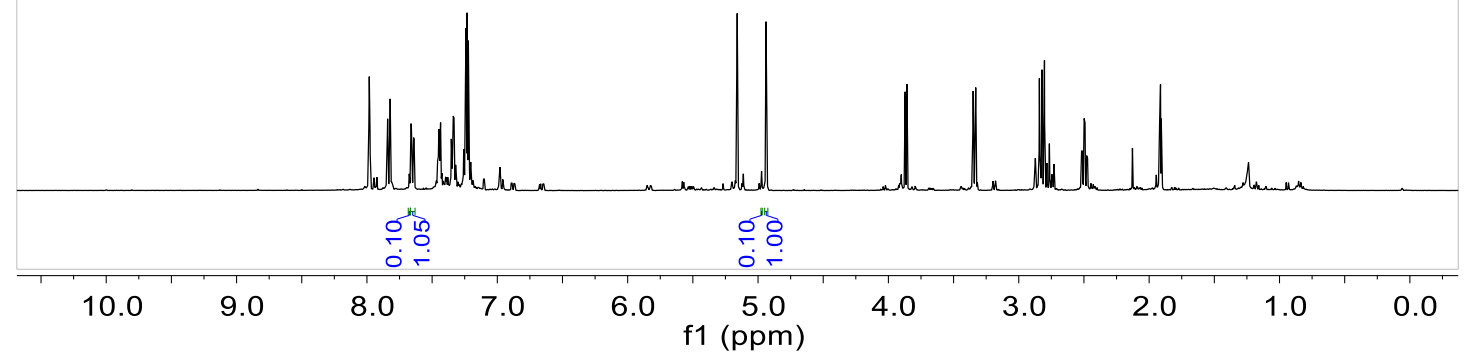




\section{X-ray Crystal Diffraction Data for 3fa}<smiles>[AlH2]</smiles>

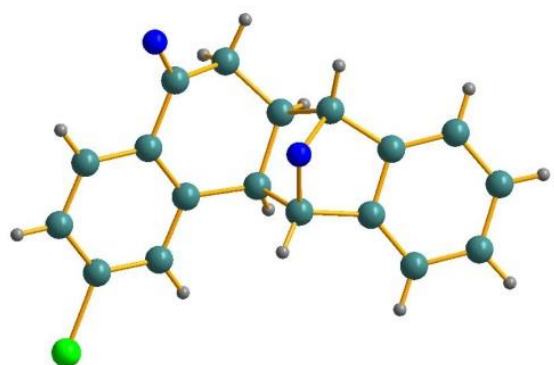<smiles>O=C1CC2OC(c3ccccc32)[C@@H]2c3cc(Cl)ccc3C(=O)C12</smiles>

:

\begin{tabular}{|l|l|}
\hline CCDC & 1940088 \\
\hline Empirical formula, Formula weight & $\mathrm{C}_{18} \mathrm{H}_{13} \mathrm{ClO}_{2}, \quad 296.73$ \\
\hline Temperature, Wavelength & $293(2) \mathrm{K}, \quad 0.71073 \AA$ \\
\hline Crystal system, space group & Monoclinic, $\mathrm{P} 2_{1} / \mathrm{n}$ \\
\hline Unit cell dimensions & $\mathrm{a}=7.245 \AA \quad$ alpha $=90 \mathrm{deg}$. \\
& $\mathrm{b}=8.425 \AA \quad$ beta $=91.79 \mathrm{deg}$. \\
& $\mathrm{c}=22.618 \AA \quad$ gamma $=90 \mathrm{deg}$. \\
\hline Volume & $1379.9 \AA \wedge 3$ \\
\hline Z, Calculated density & $4,1.428 \mathrm{Mg} / \mathrm{m}^{\wedge} 3$ \\
\hline Absorption coefficient & $0.278 \mathrm{~mm}^{\wedge}-1$ \\
\hline F(000) & 616.0 \\
\hline Theta range for data collection & 3.604 to $50.75 \mathrm{deg}$. \\
\hline Reflections collected / unique & $20084 / 2500\left[\mathrm{R}_{\text {int }}=0.0368, \mathrm{R}_{\text {sigma }}=0.0216\right]$ \\
\hline Completeness to theta $=25.375$ & $98.6 \%$ \\
\hline Absorption correction & $\mathrm{MULTI}-\mathrm{SCAN}$ \\
\hline Data / restraints / parameters & $2500 / 0 / 190$ \\
\hline Goodness-of-fit on $\mathrm{F}^{\wedge} 2$ & 1.079 \\
\hline Final R indices [I>2sigma(I) $]$ & $\mathrm{R}_{1}=0.0328, \mathrm{wR}_{2}=0.0826$ \\
\hline R indices (all data) & $\mathrm{R}_{1}=0.0331, \mathrm{wR}_{2}=0.0828$ \\
\hline Largest diff. peak and hole & 0.23 and $-0.20 \mathrm{e} . \AA \wedge \wedge$ \\
\hline
\end{tabular}




\section{References}

(1). Vaitla, J.; Bayer, A.; Hopmann, K. H. Angew., Chem., Int. Ed. 2017, 56, 4277.

(2). Gandeepan, P.; Rajamalli, P.; Cheng, C.-H. Angew., Chem. Int. Ed. 2016, 55, 4308.

(3). Lu, Z.; Zhang, H.; Yang, Z.; Ding, N.; Meng, L.; Wang, J. ACS Catal. 2019, 9, 1457. 
VIII. ${ }^{1} \mathrm{H},{ }^{13} \mathrm{C}$ NMR Spectra of New Compounds.

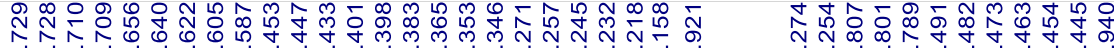
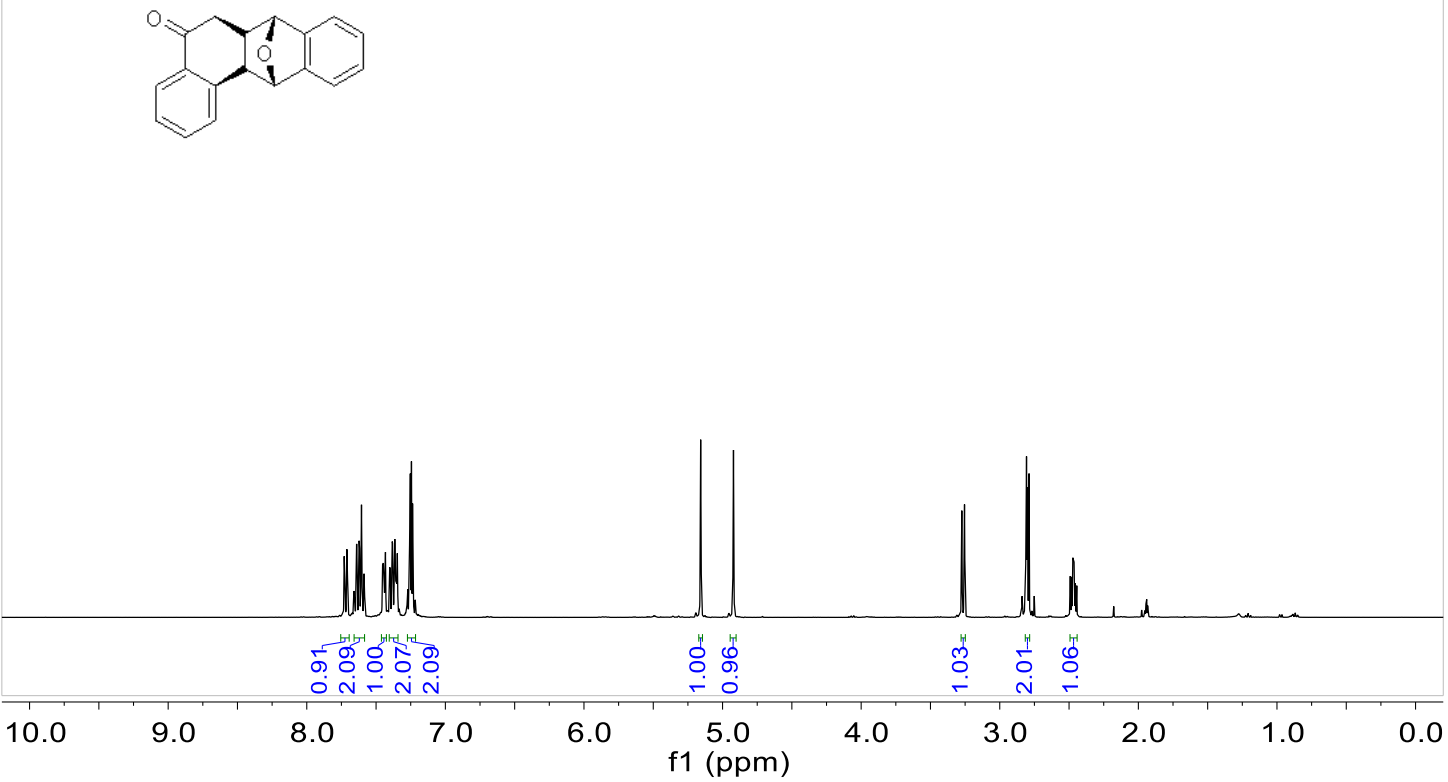

${ }^{1} \mathrm{H}$ NMR (400 MHz, $\left.\mathrm{CD}_{3} \mathrm{CN}\right)$ spectrum of 3aa

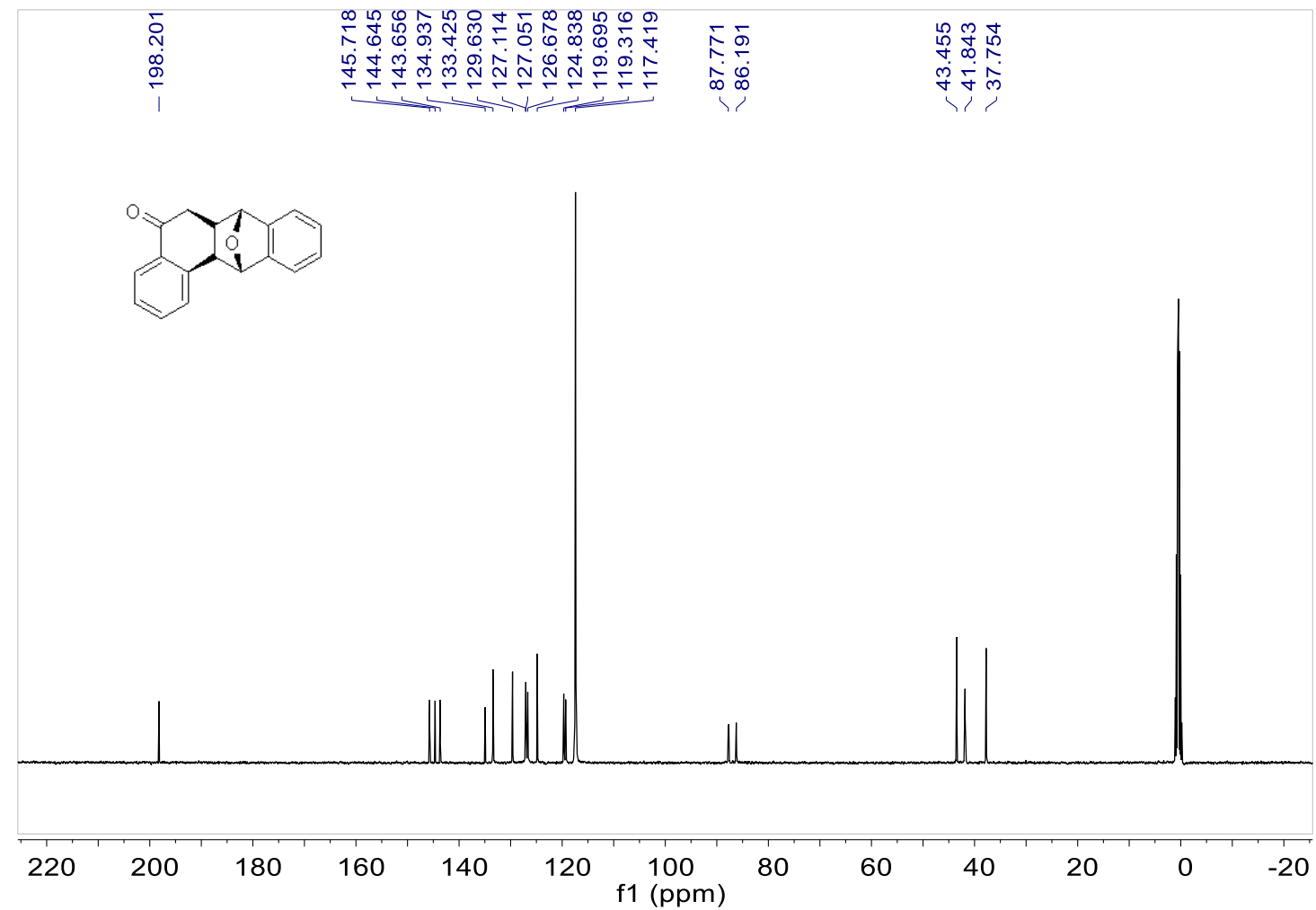

${ }^{13} \mathrm{C}$ NMR (100 MHz, $\left.\mathrm{CD}_{3} \mathrm{CN}\right)$ spectrum of $3 \mathrm{aa}$ 


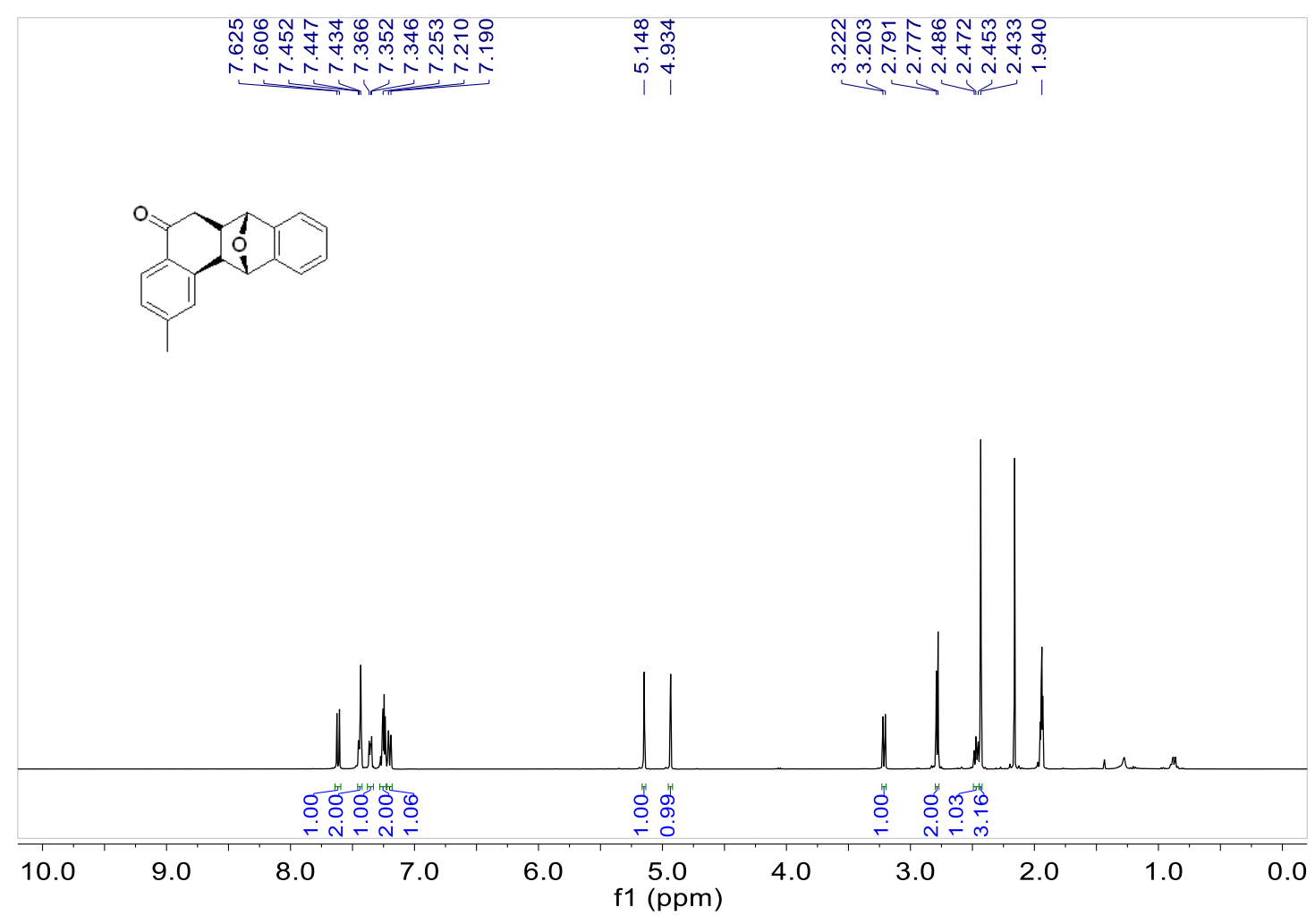

${ }^{1} \mathrm{H}$ NMR (400 MHz, CD $\mathrm{CN}$ ) spectrum of $3 \mathrm{ba}$

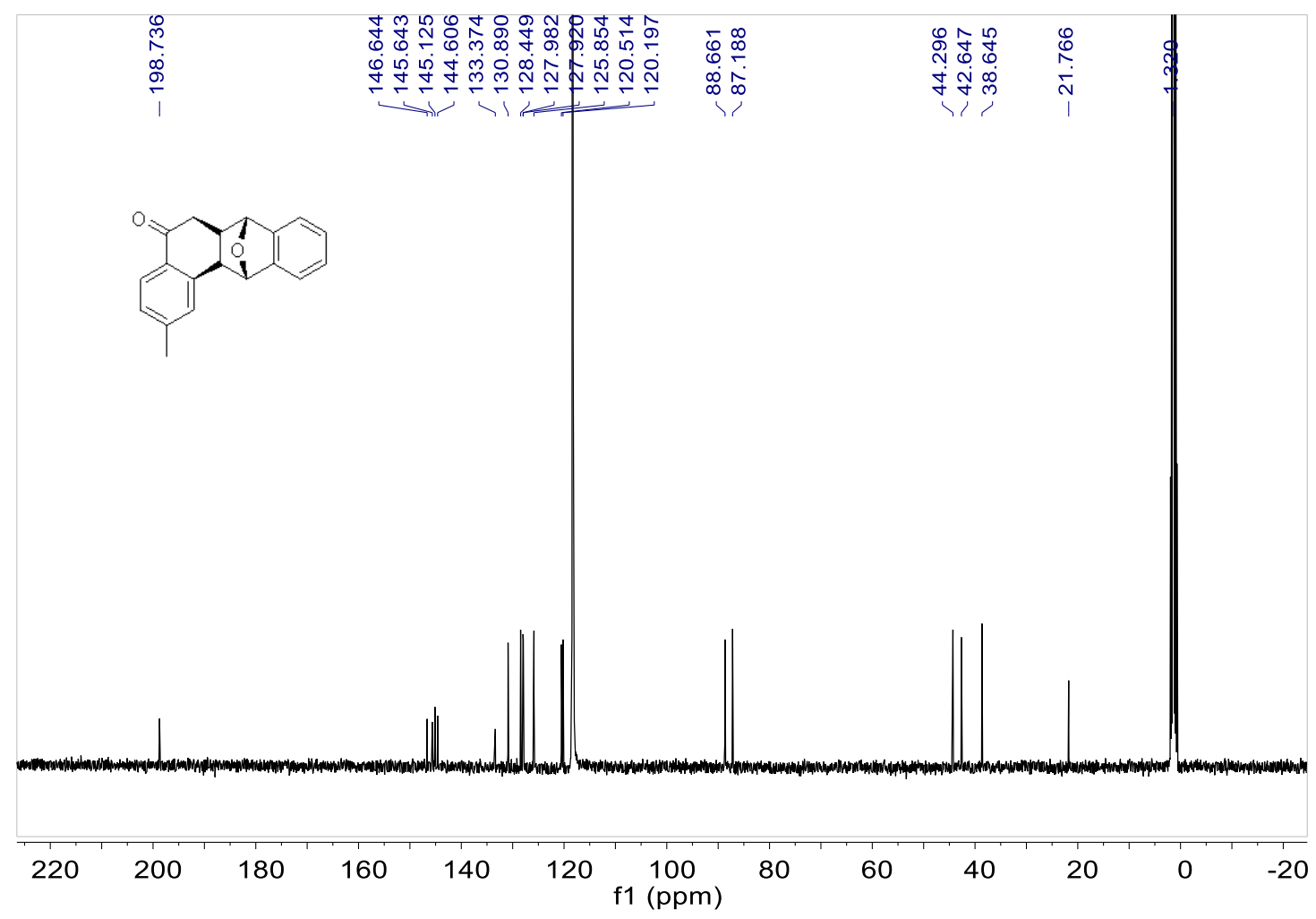

${ }^{13} \mathrm{C}$ NMR (100 MHz, $\left.\mathrm{CD}_{3} \mathrm{CN}\right)$ spectrum of $3 \mathrm{ba}$ 


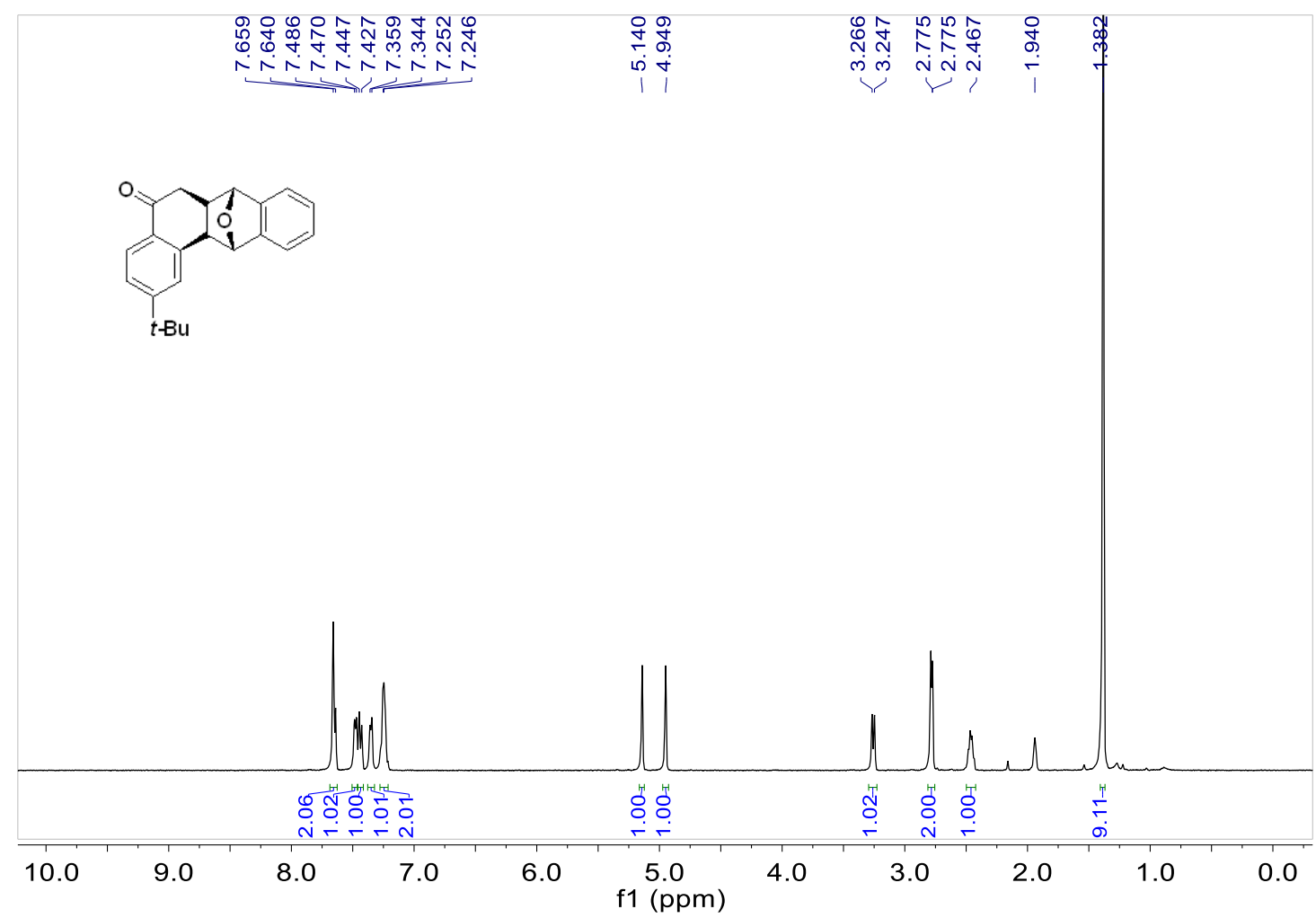

${ }^{1} \mathrm{H}$ NMR (400 MHz, $\mathrm{CD}_{3} \mathrm{CN}$ ) spectrum of 3ca

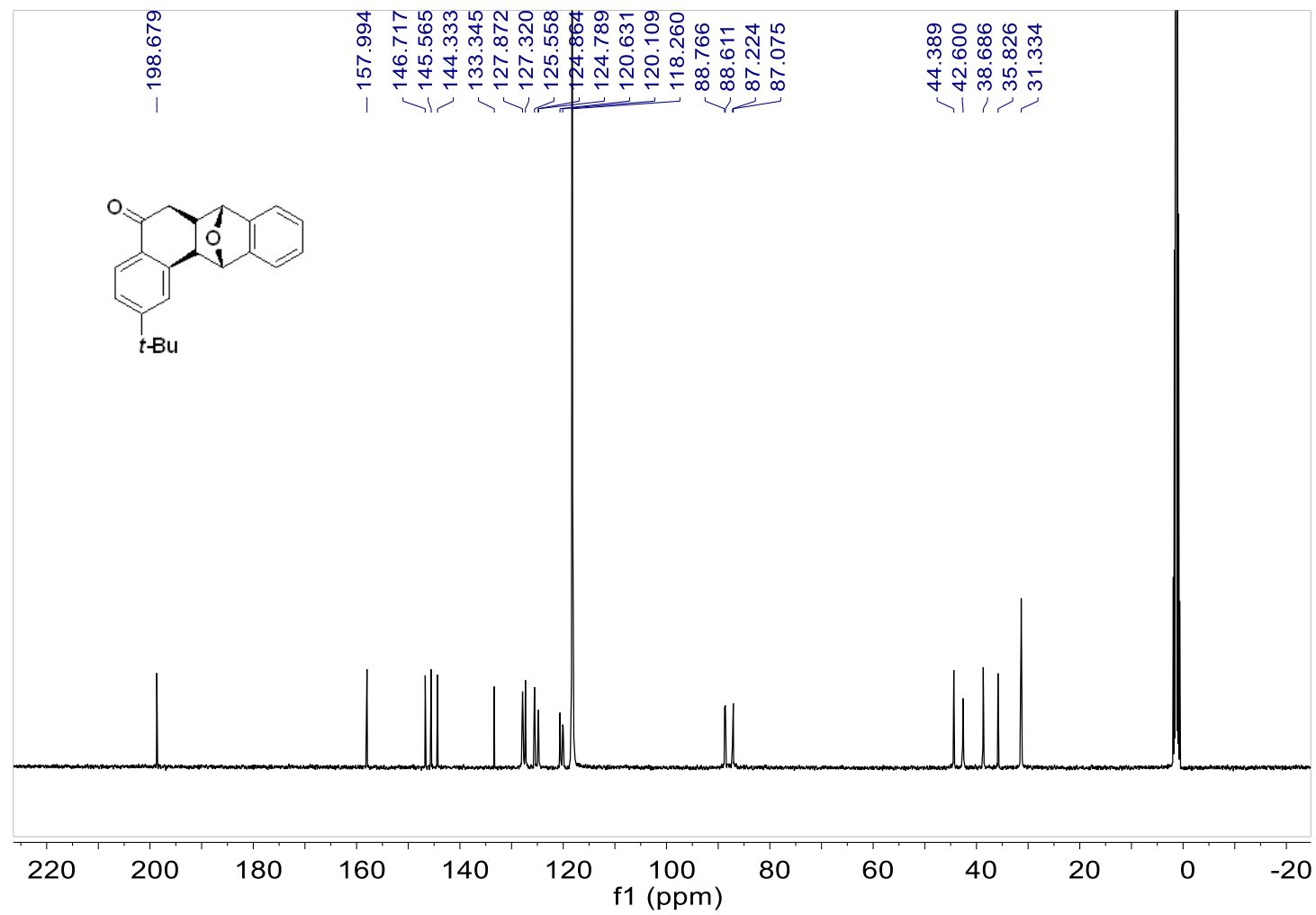

${ }^{13} \mathrm{C}$ NMR (100 MHz, CD 3 CN) spectrum of $3 \mathrm{ca}$ 


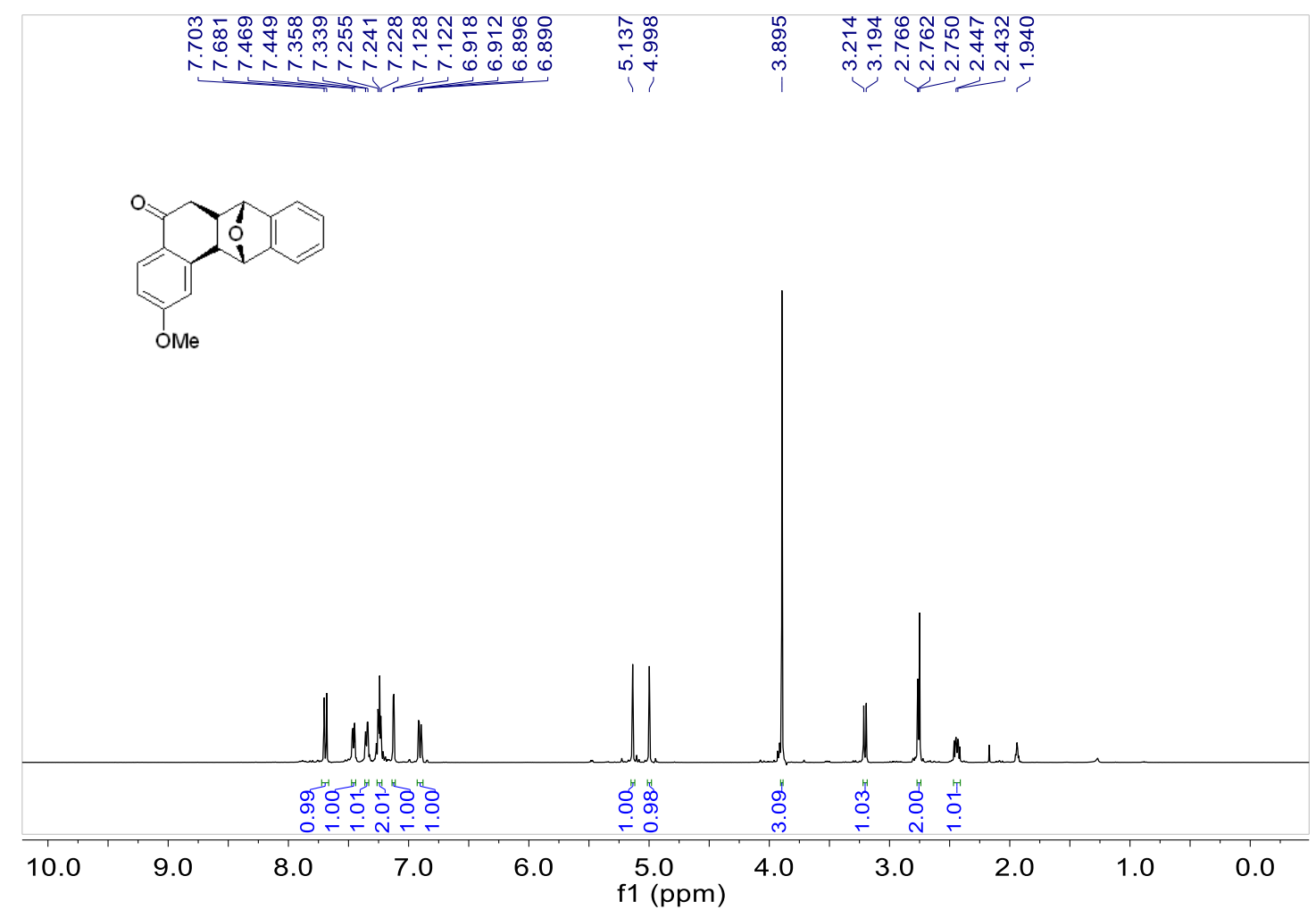

${ }^{1} \mathrm{H}$ NMR (400 MHz, $\left.\mathrm{CD}_{3} \mathrm{CN}\right)$ spectrum of $3 \mathrm{da}$

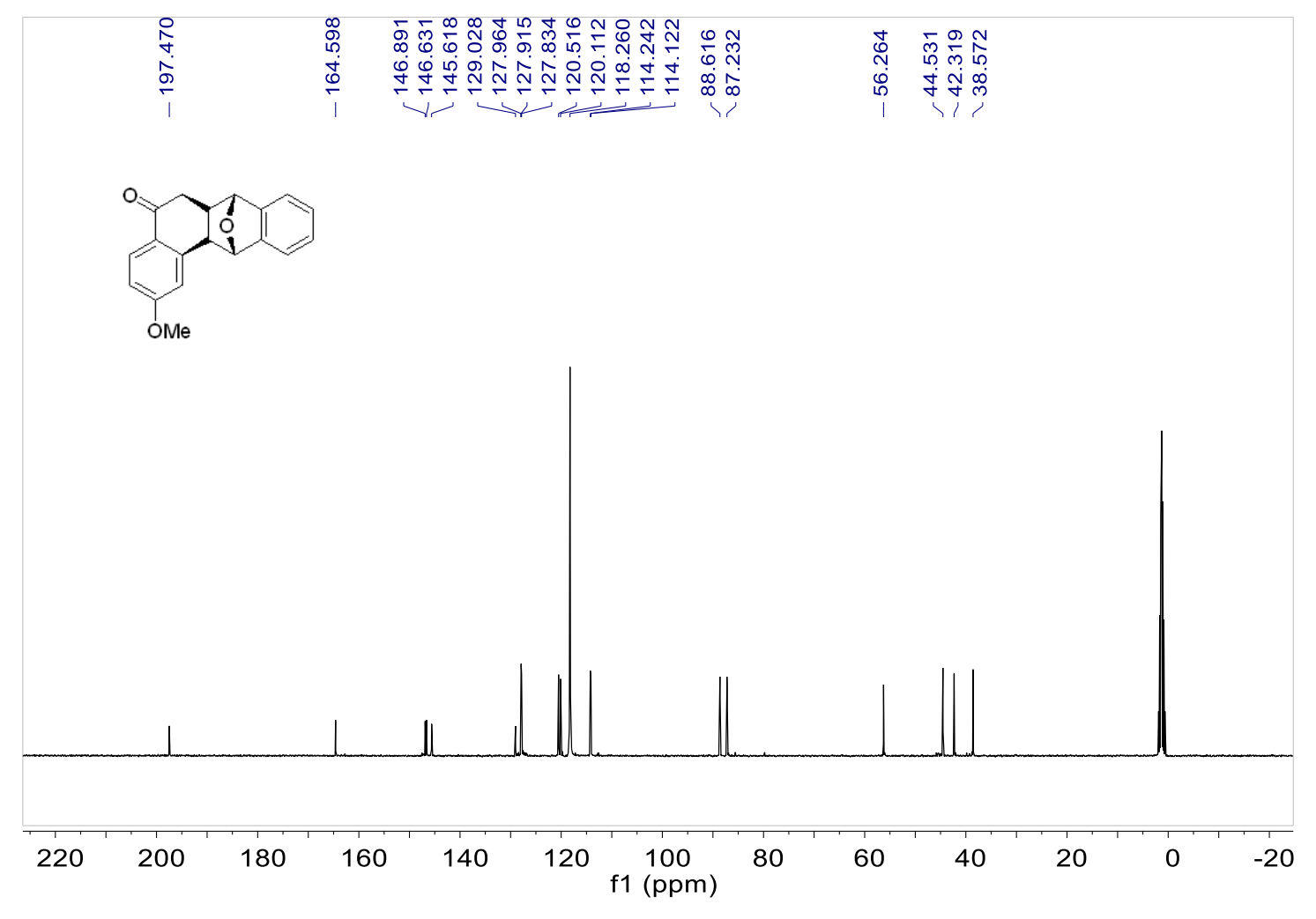

${ }^{13} \mathrm{C}$ NMR (100 MHz, $\mathrm{CD}_{3} \mathrm{CN}$ ) spectrum of $3 \mathrm{da}$ 


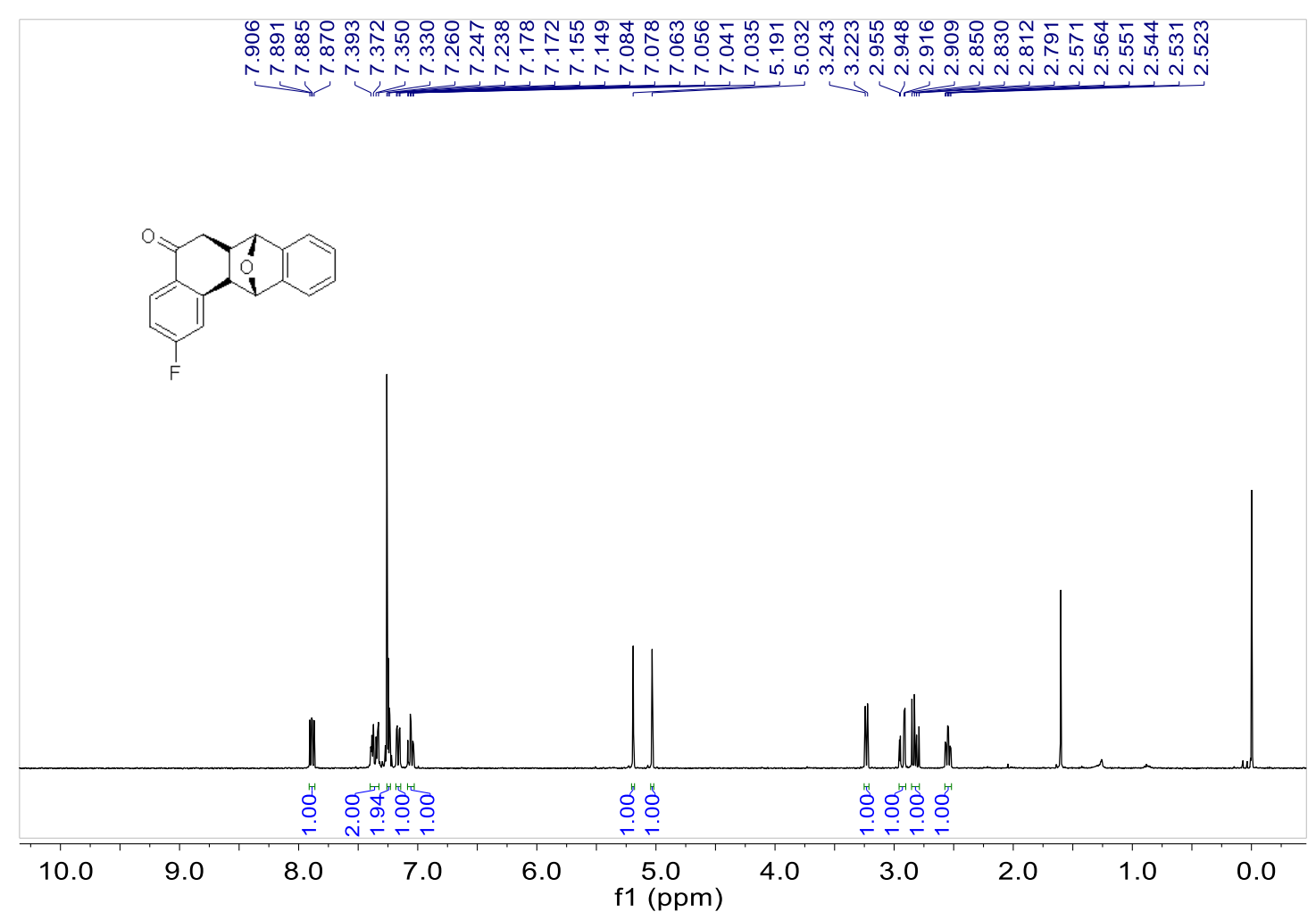

${ }^{1} \mathrm{H}$ NMR (400 MHz, $\mathrm{CDCl}_{3}$ ) spectrum of 3ea

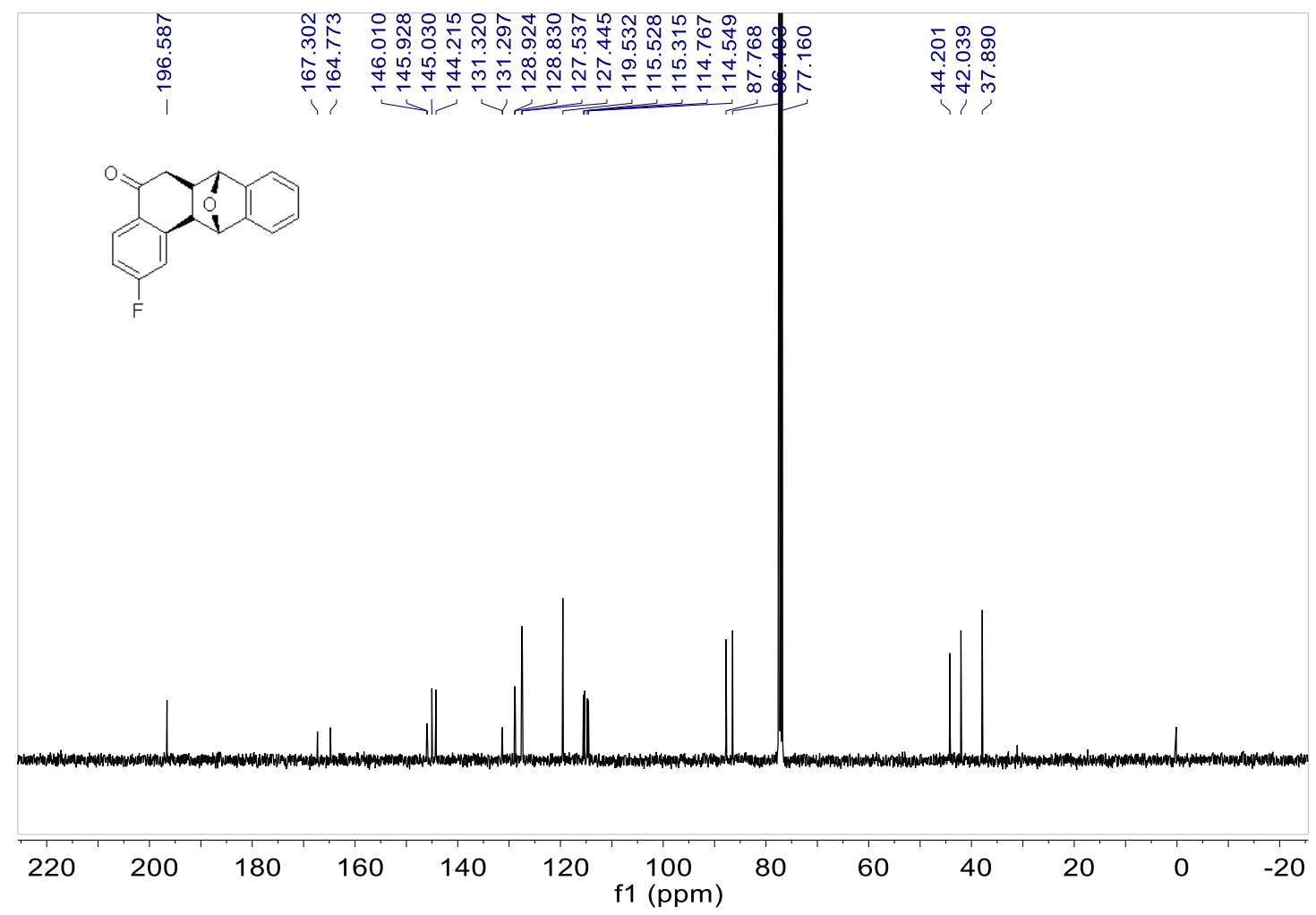

${ }^{13} \mathrm{C}$ NMR (100 $\left.\mathrm{MHz}, \mathrm{CDCl}_{3}\right)$ spectrum of 3ea 


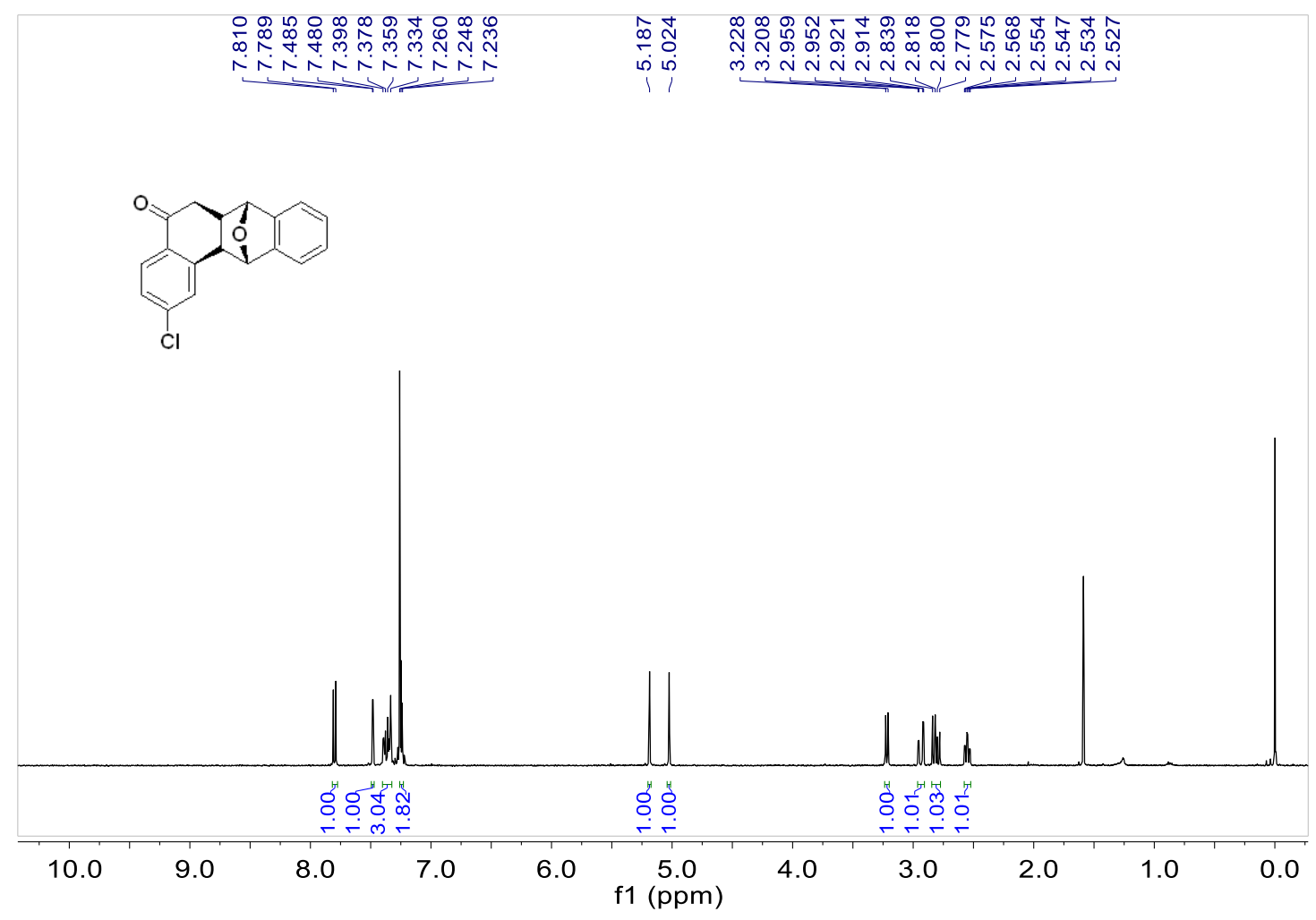

${ }^{1} \mathrm{H}$ NMR (400 MHz, $\mathrm{CDCl}_{3}$ ) spectrum of $3 f a$

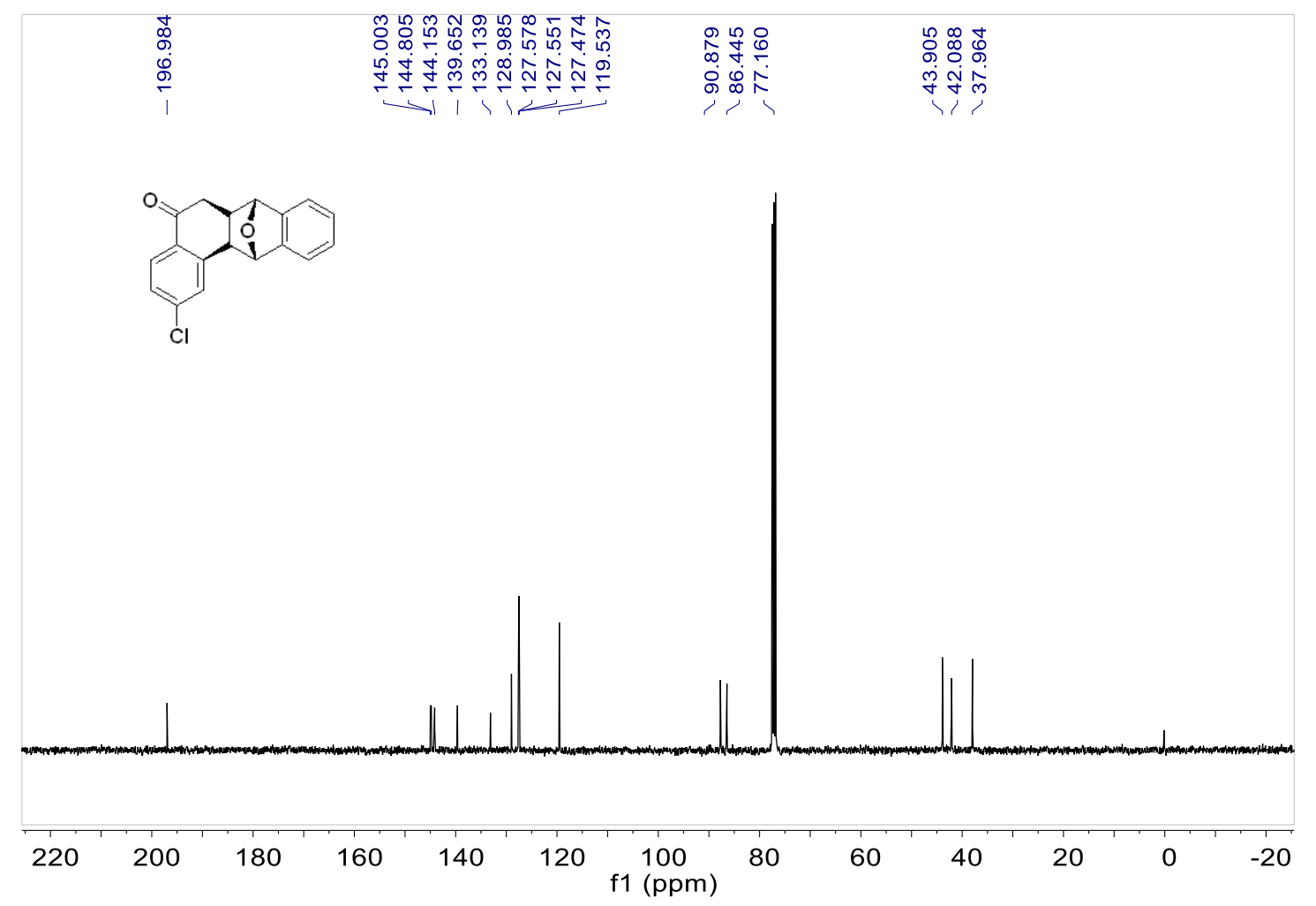

${ }^{13} \mathrm{C}$ NMR (100 MHz, $\mathrm{CDCl}_{3}$ ) spectrum of 3fa 

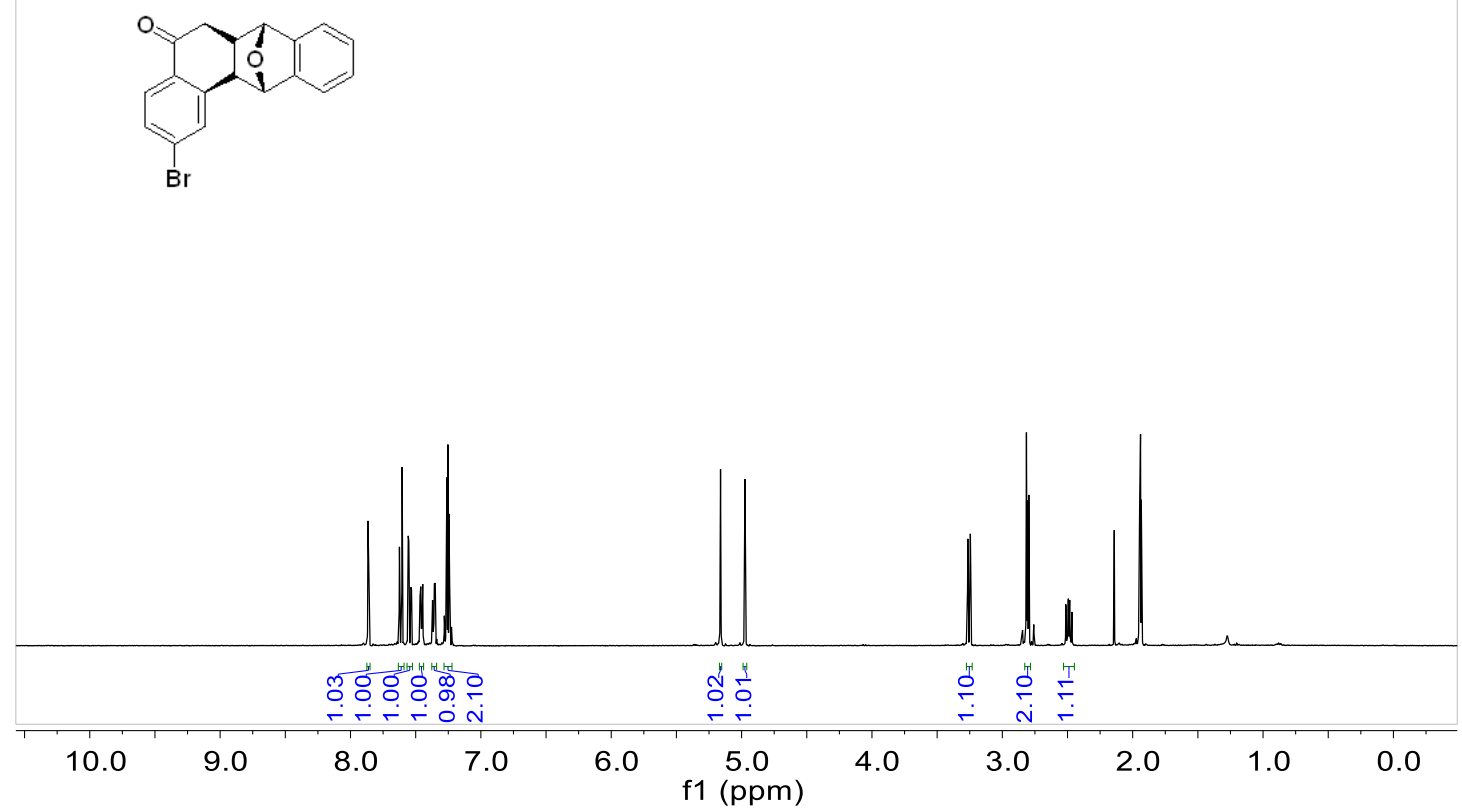

${ }^{1} \mathrm{H}$ NMR (400 MHz, $\left.\mathrm{CD}_{3} \mathrm{CN}\right)$ spectrum of $3 \mathrm{ga}$

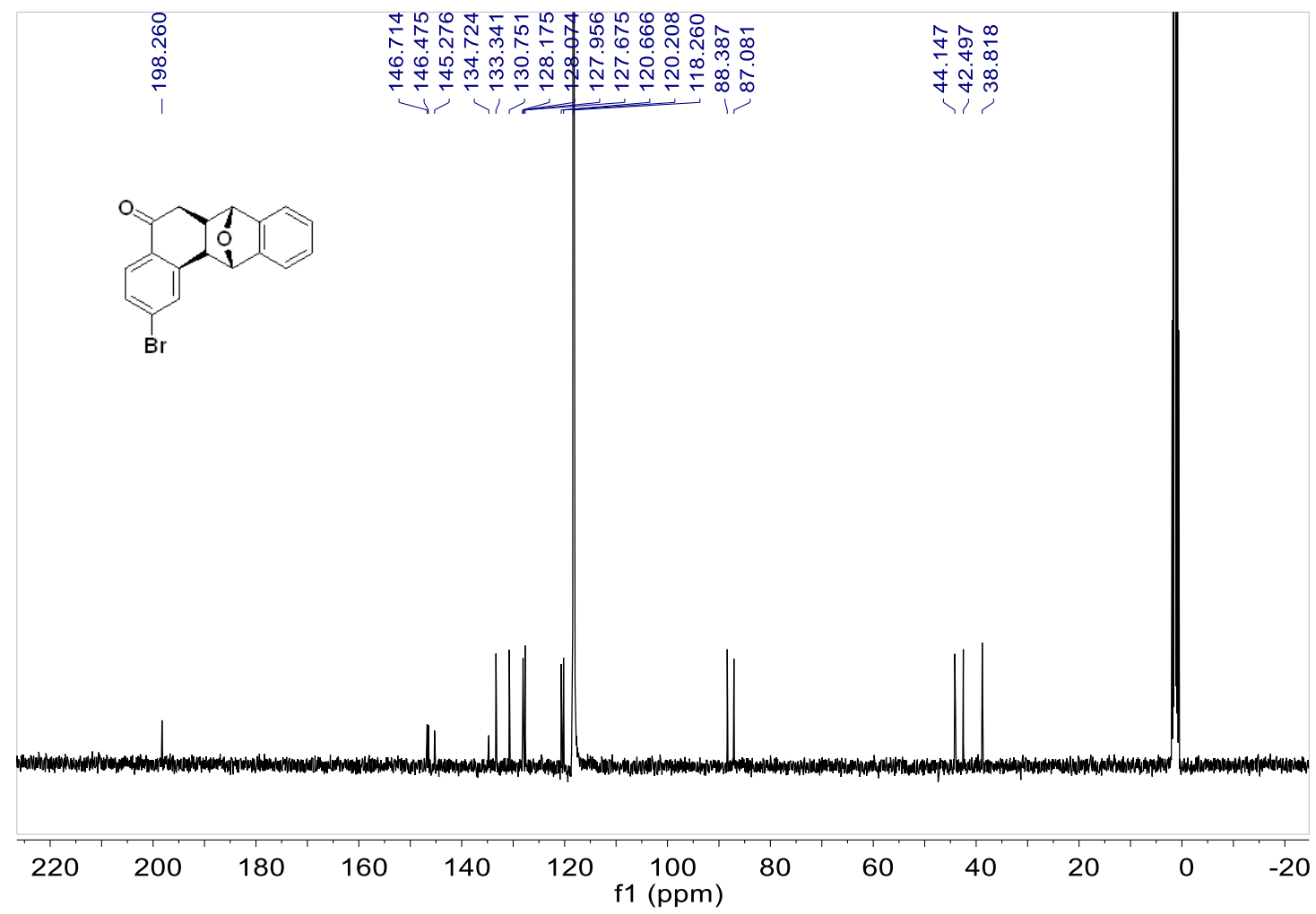

${ }^{13} \mathrm{C}$ NMR (100 MHz, $\left.\mathrm{CD}_{3} \mathrm{CN}\right)$ spectrum of 3ga 

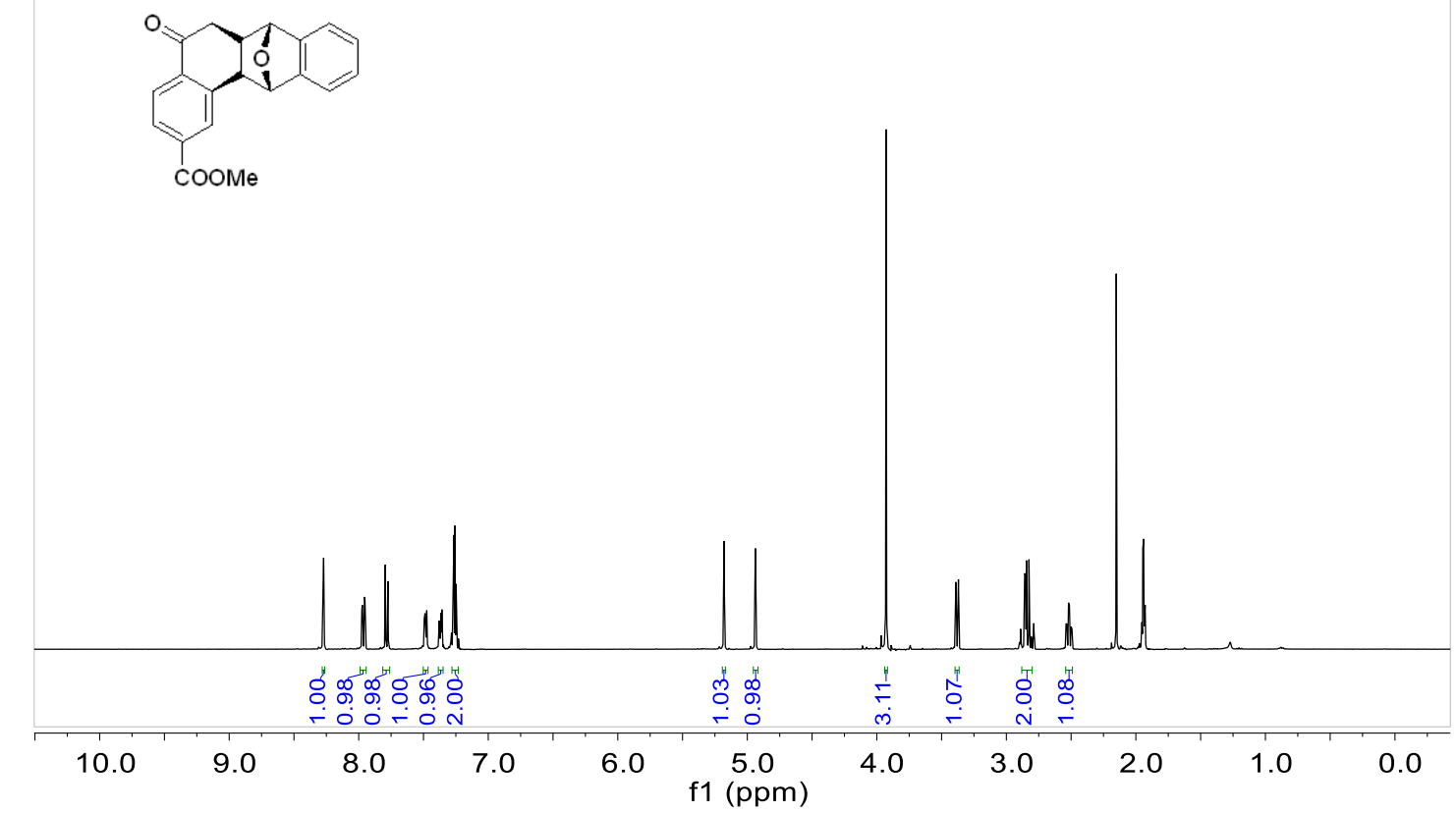

${ }^{1} \mathrm{H}$ NMR (400 MHz, $\left.\mathrm{CD}_{3} \mathrm{CN}\right)$ spectrum of $3 \mathrm{ha}$

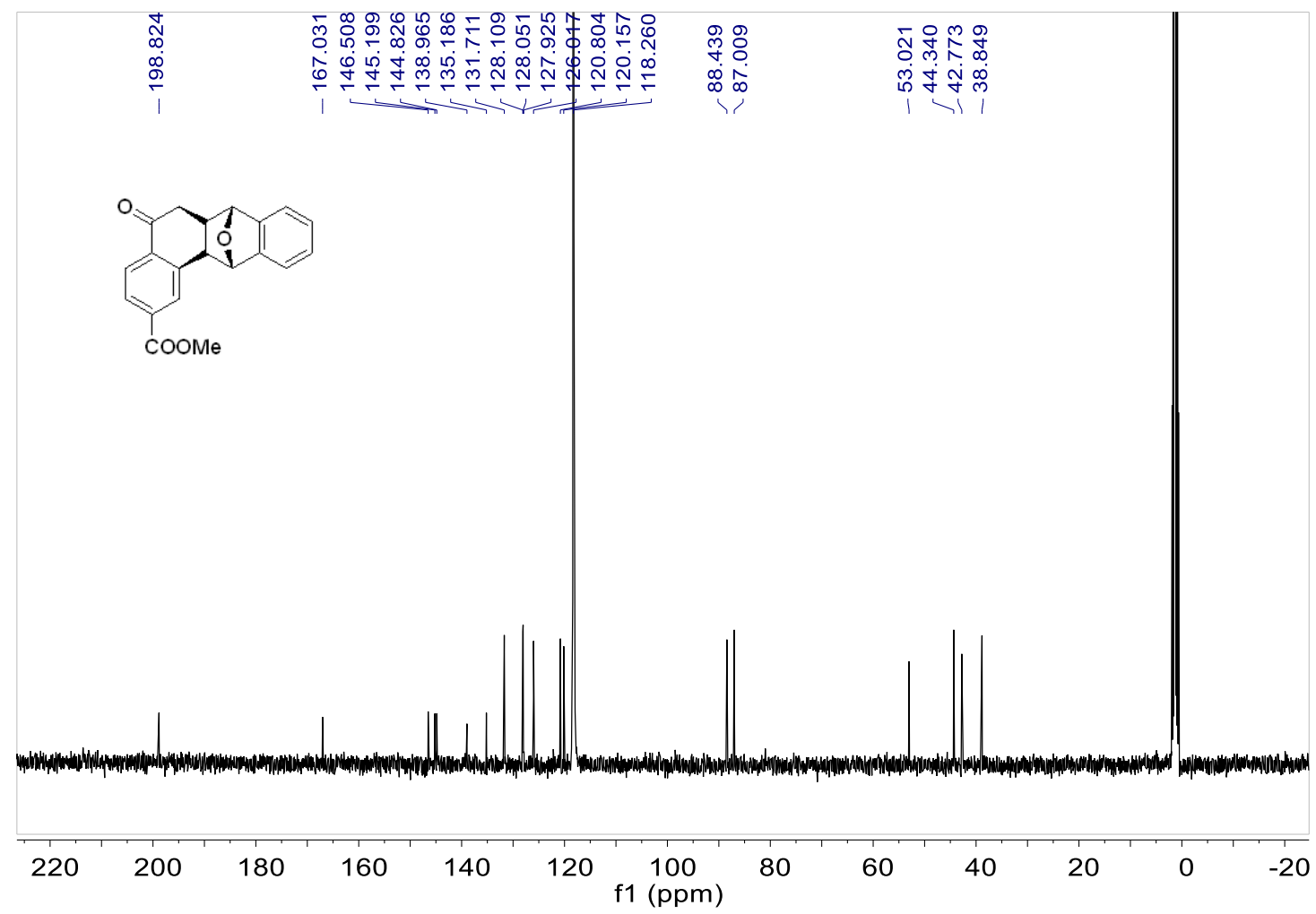

${ }^{13} \mathrm{C}$ NMR (100 MHz, CD $\mathrm{CDN}_{3} \mathrm{Cpectrum}$ of $3 \mathrm{ha}$ 

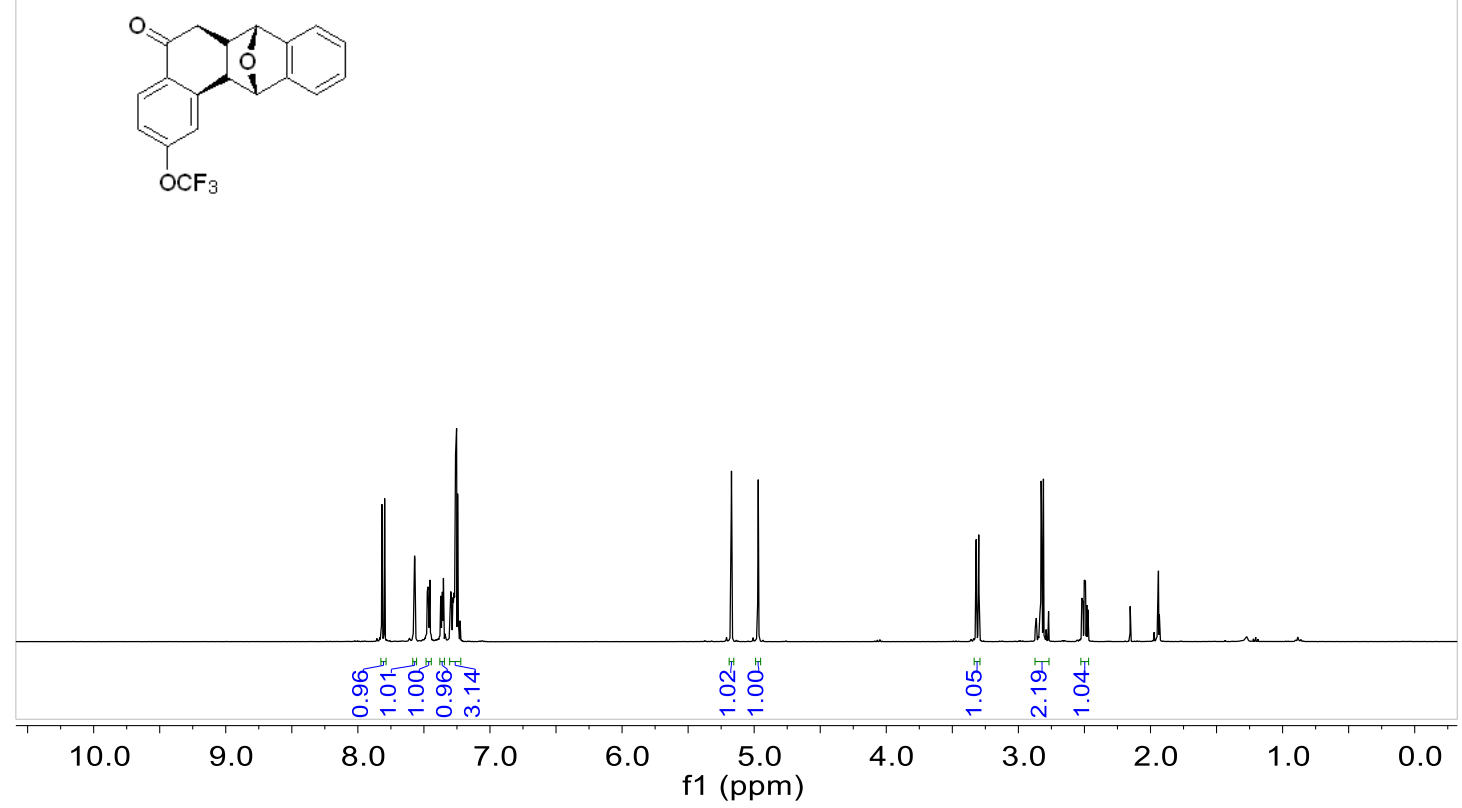

${ }^{1} \mathrm{H}$ NMR (400 MHz, CD $3 \mathrm{CN}$ ) spectrum of 3ia
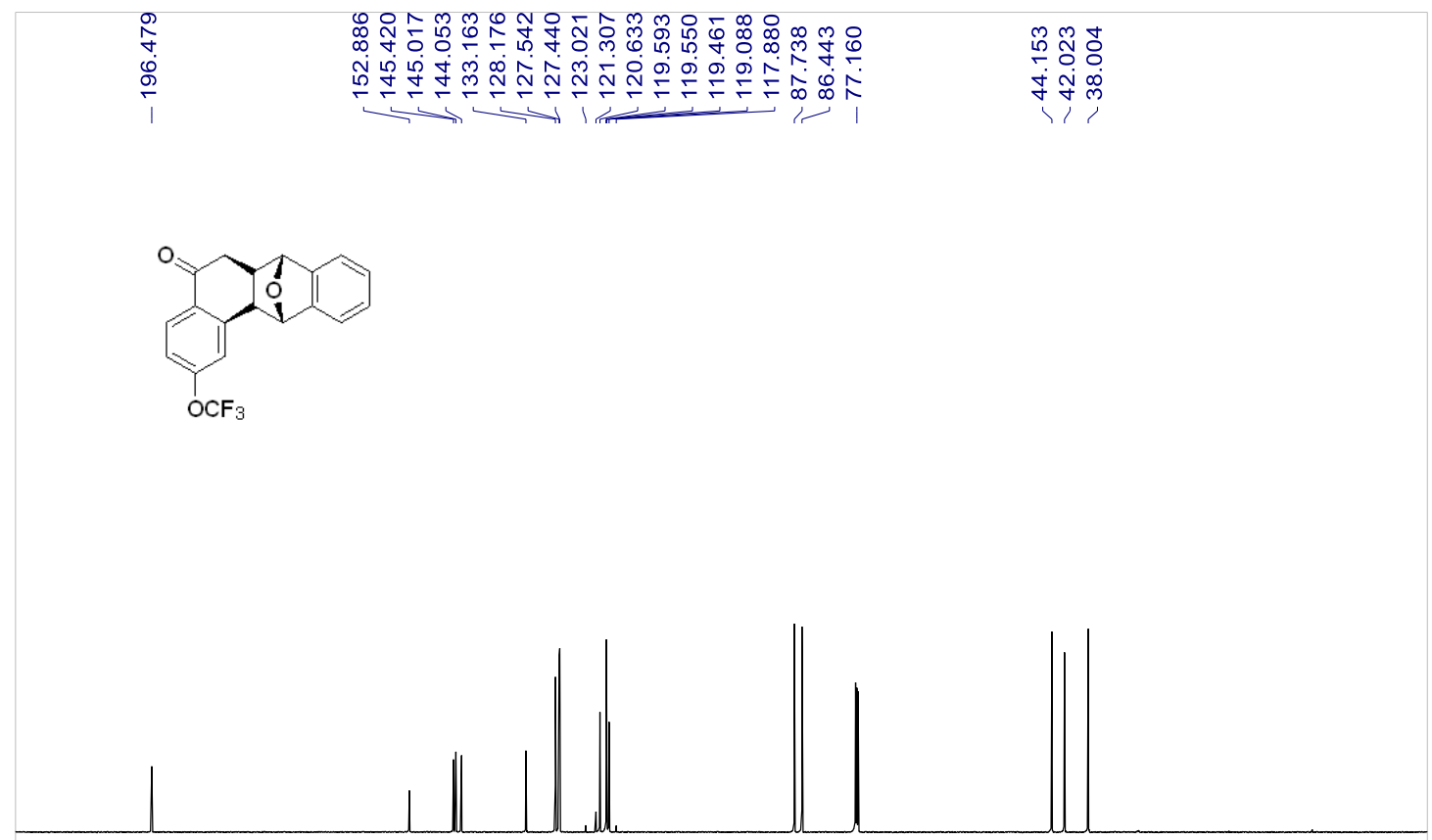

$21020019018017016015014013012011010090 \quad 80 \quad 70 \quad 60 \quad 50 \quad 40 \quad 30 \quad 20 \quad 10 \quad 0 \quad-10$ f1 (ppm)

${ }^{13} \mathrm{C}$ NMR (150 MHz, $\left.\mathrm{CDCl}_{3}\right)$ spectrum of 3ia 

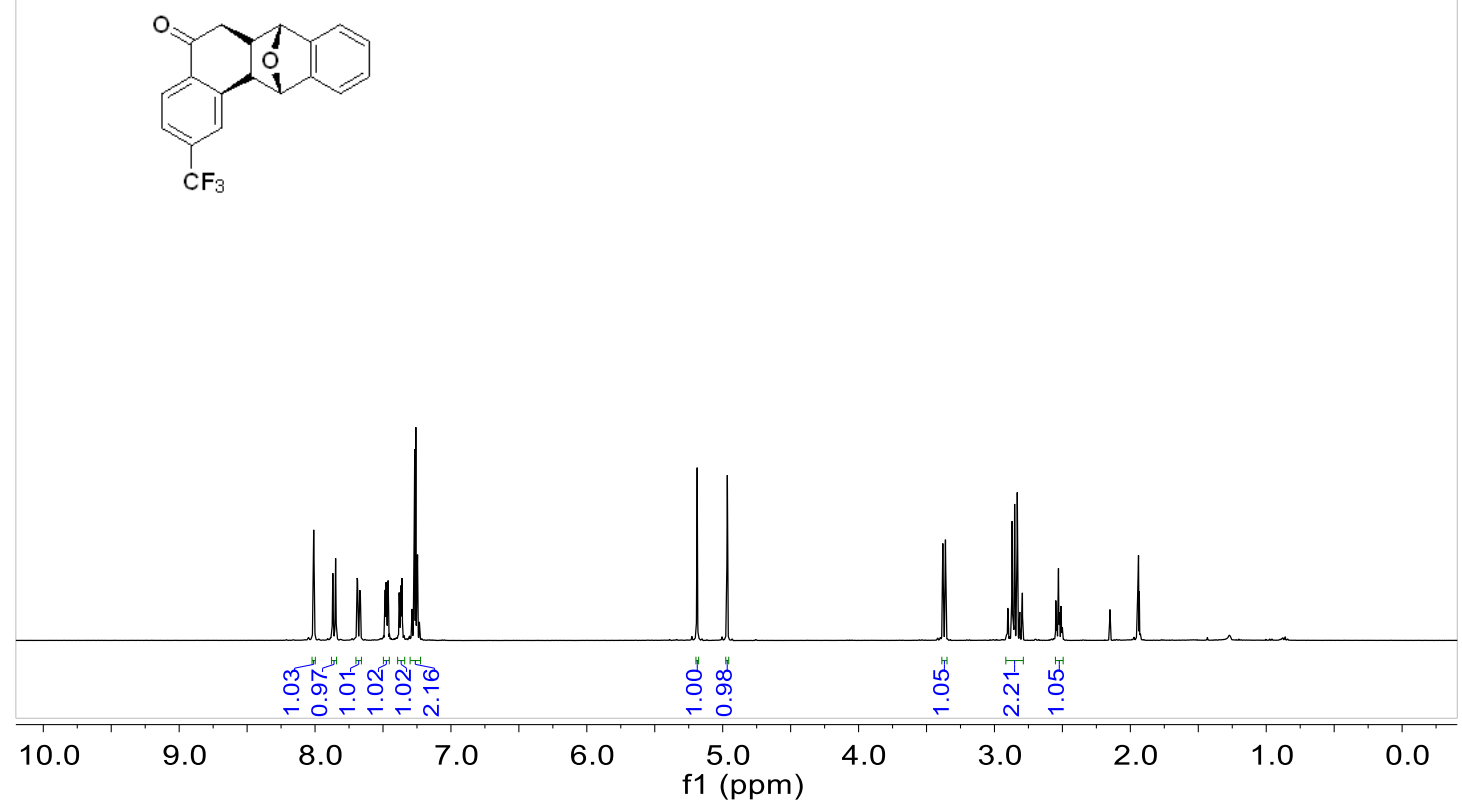

${ }^{1} \mathrm{H}$ NMR (400 MHz, $\left.\mathrm{CD}_{3} \mathrm{CN}\right)$ spectrum of $3 \mathrm{ja}$

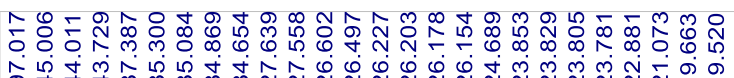

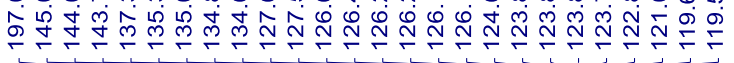

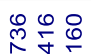

舟究

$\stackrel{2}{\circ} \stackrel{2}{\circ} \circ$

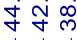<smiles></smiles>

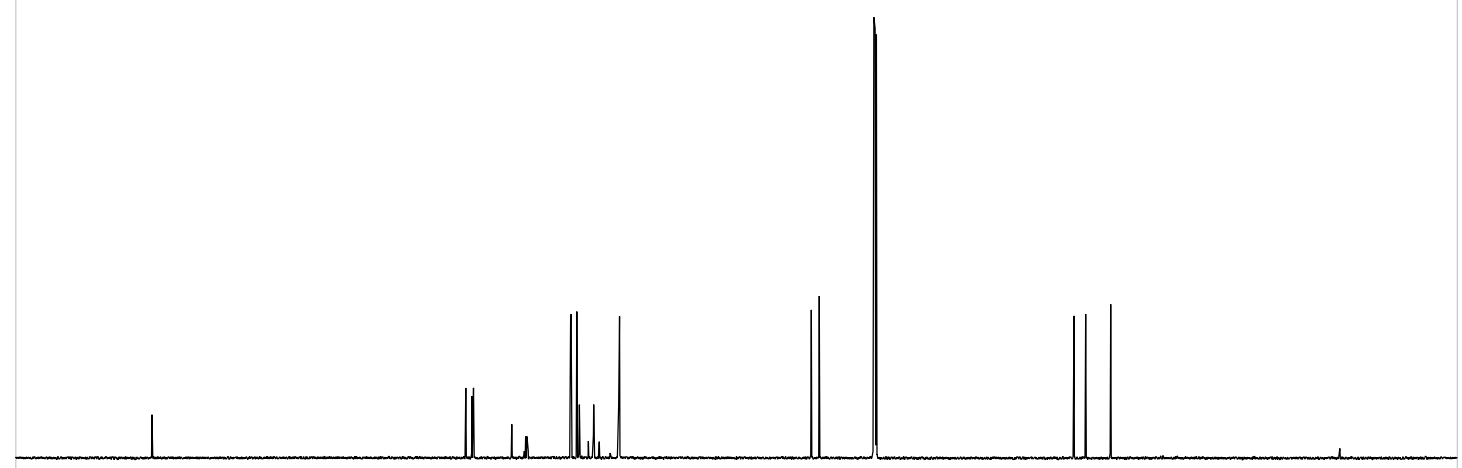

$21020019018017016015014013012011010090 \quad 80 \quad 70 \quad 60 \quad 50 \quad 40 \quad 30 \quad 20 \quad 10 \quad 0 \quad-10$ f1 (ppm)

${ }^{13} \mathrm{C}$ NMR (150 MHz, $\left.\mathrm{CDCl}_{3}\right)$ spectrum of $3 \mathrm{ja}$ 


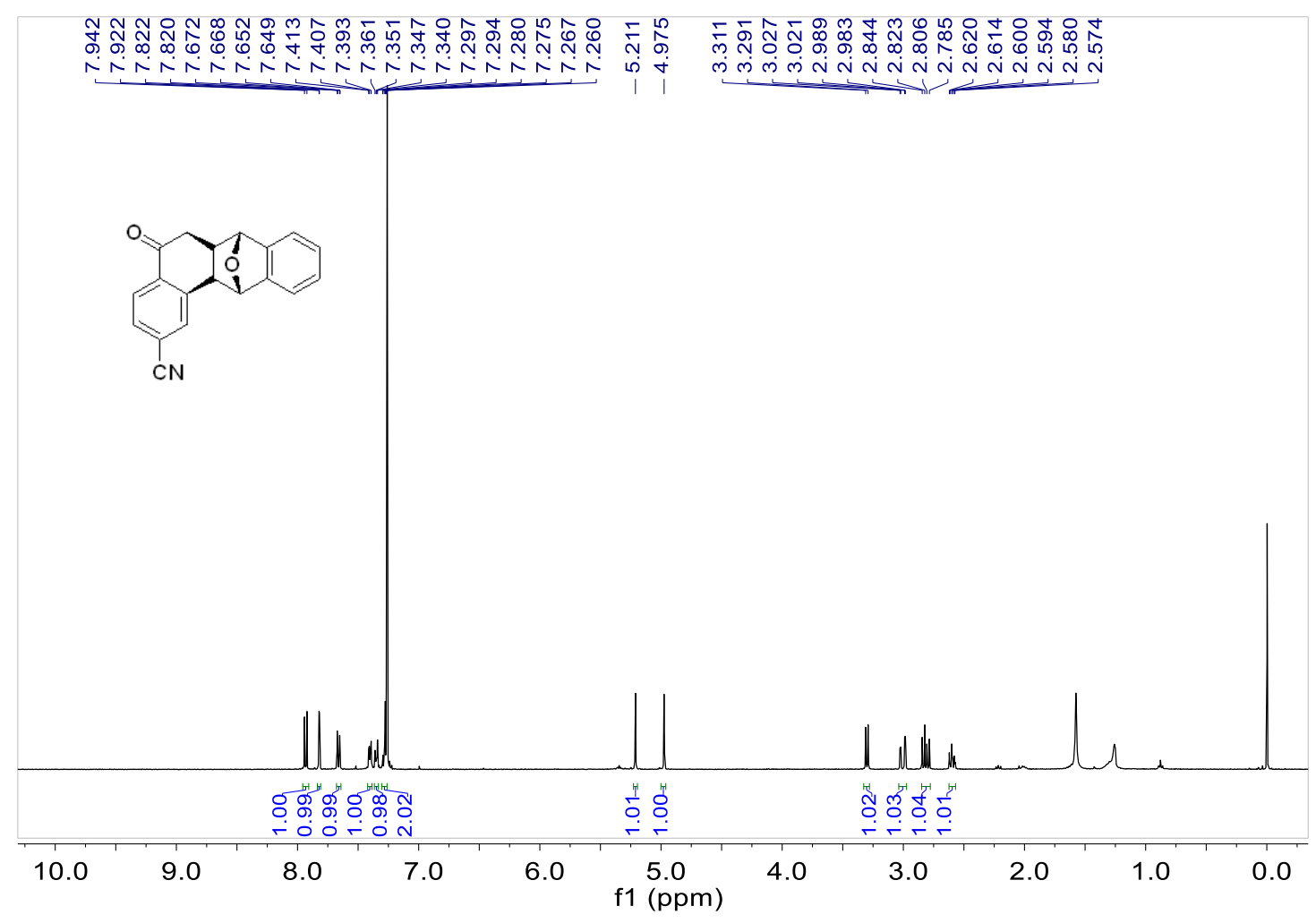

${ }^{1} \mathrm{H}$ NMR (400 MHz, $\mathrm{CDCl}_{3}$ ) spectrum of $3 \mathrm{ka}$

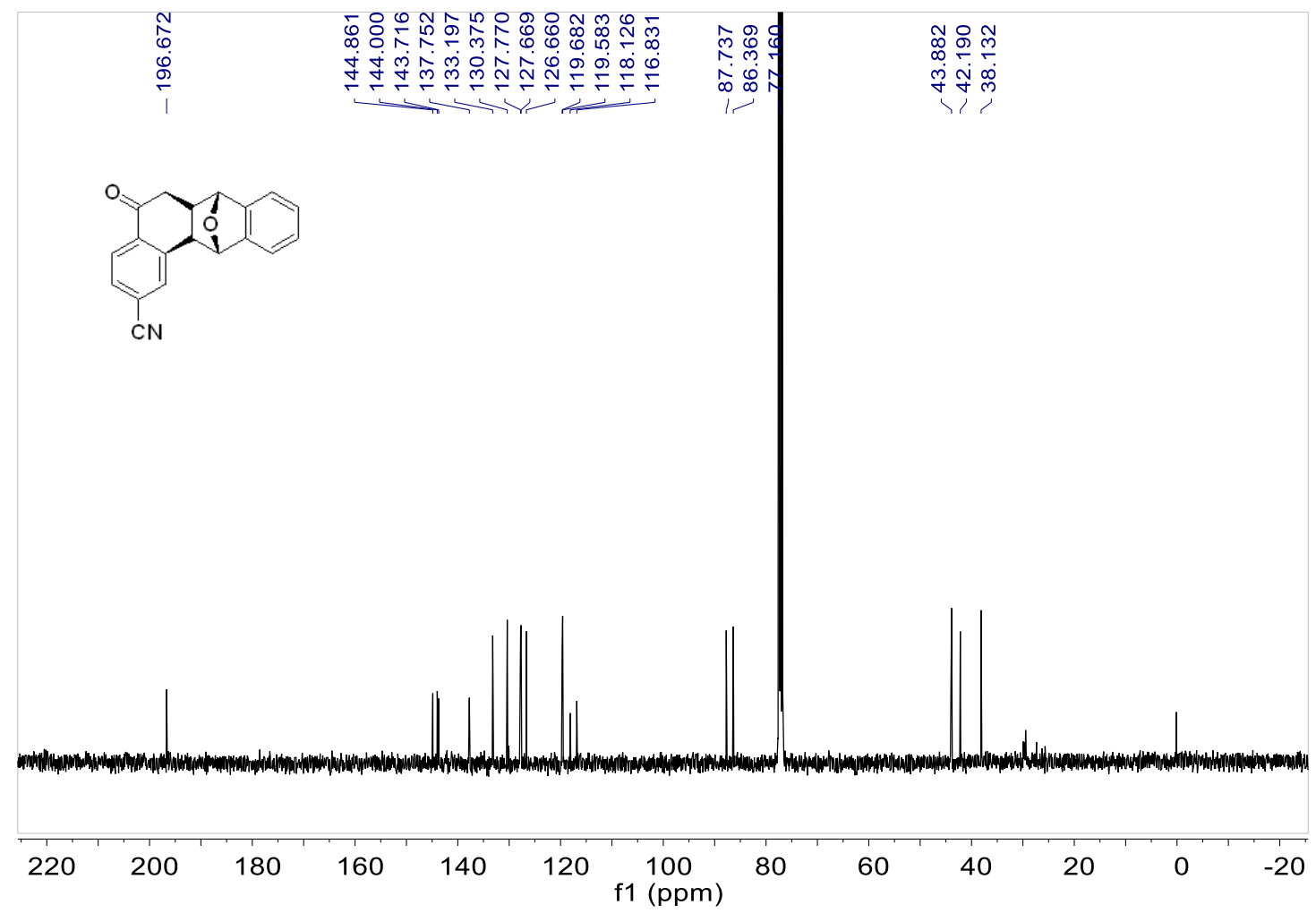

${ }^{13} \mathrm{C}$ NMR (100 MHz, $\mathrm{CDCl}_{3}$ ) spectrum of 3ka 


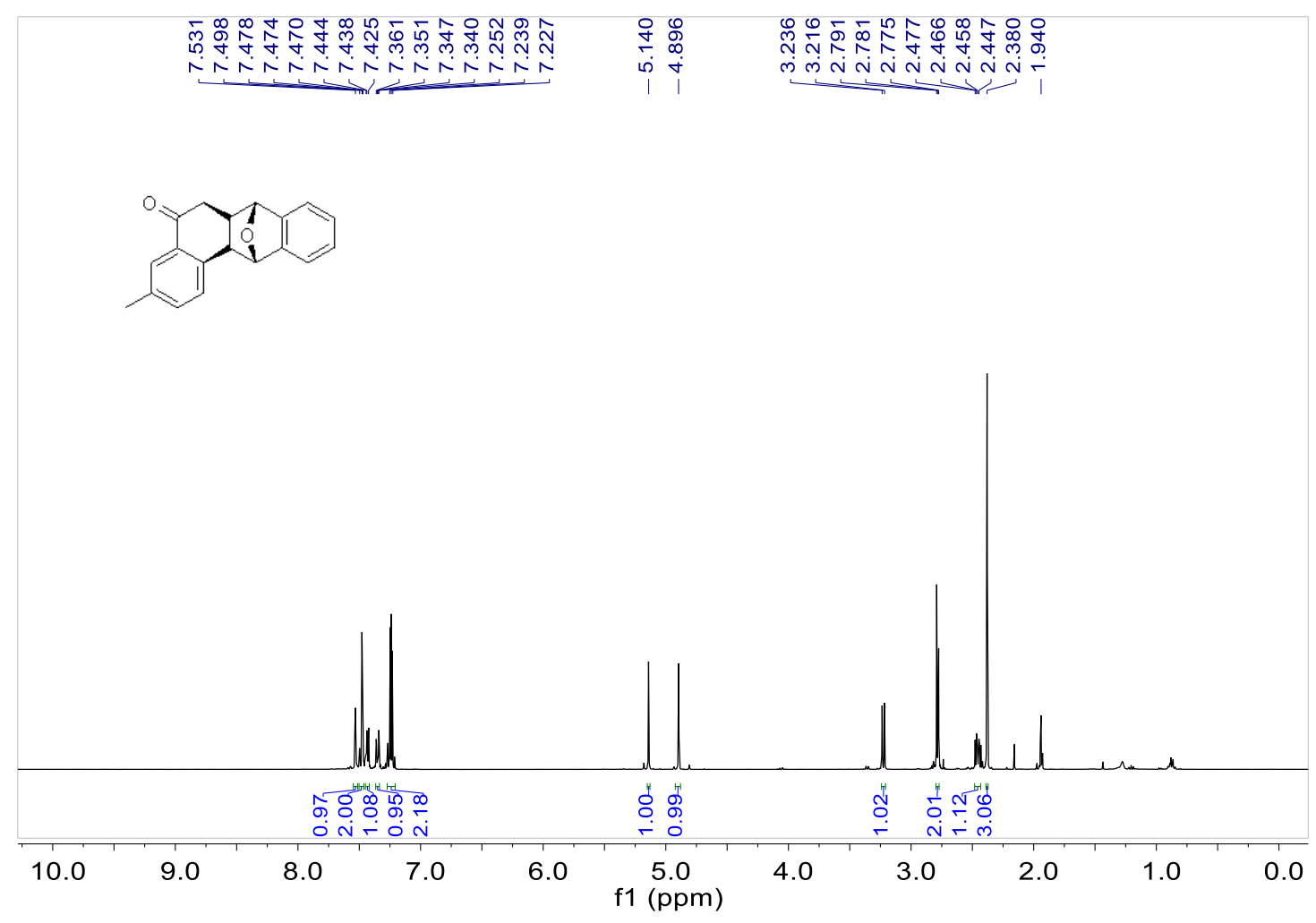

${ }^{1} \mathrm{H}$ NMR (400 MHz, $\mathrm{CD}_{3} \mathrm{CN}$ ) spectrum of 3la

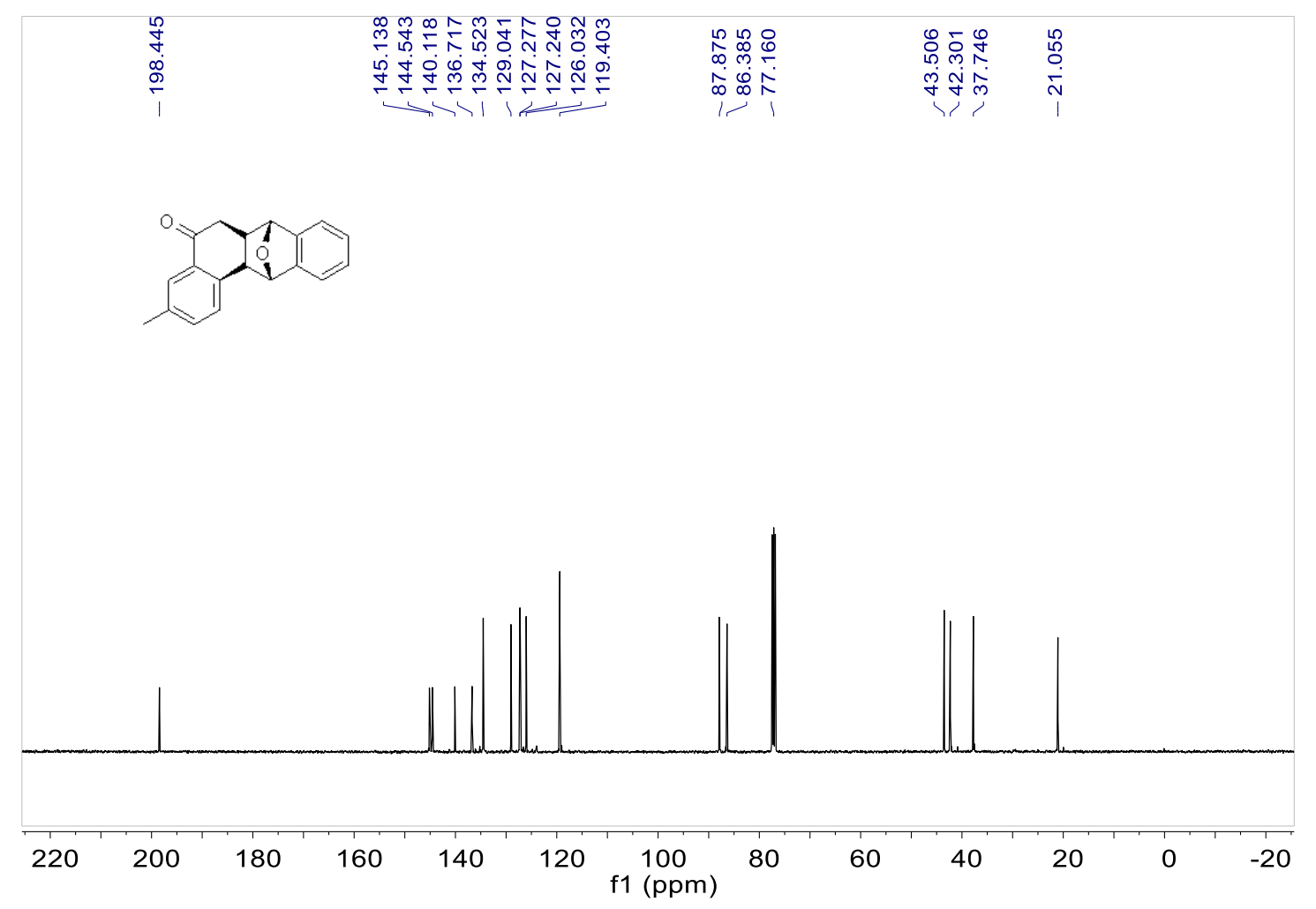

${ }^{13} \mathrm{C}$ NMR (100 MHz, $\mathrm{CDCl}_{3}$ ) spectrum of 3la 

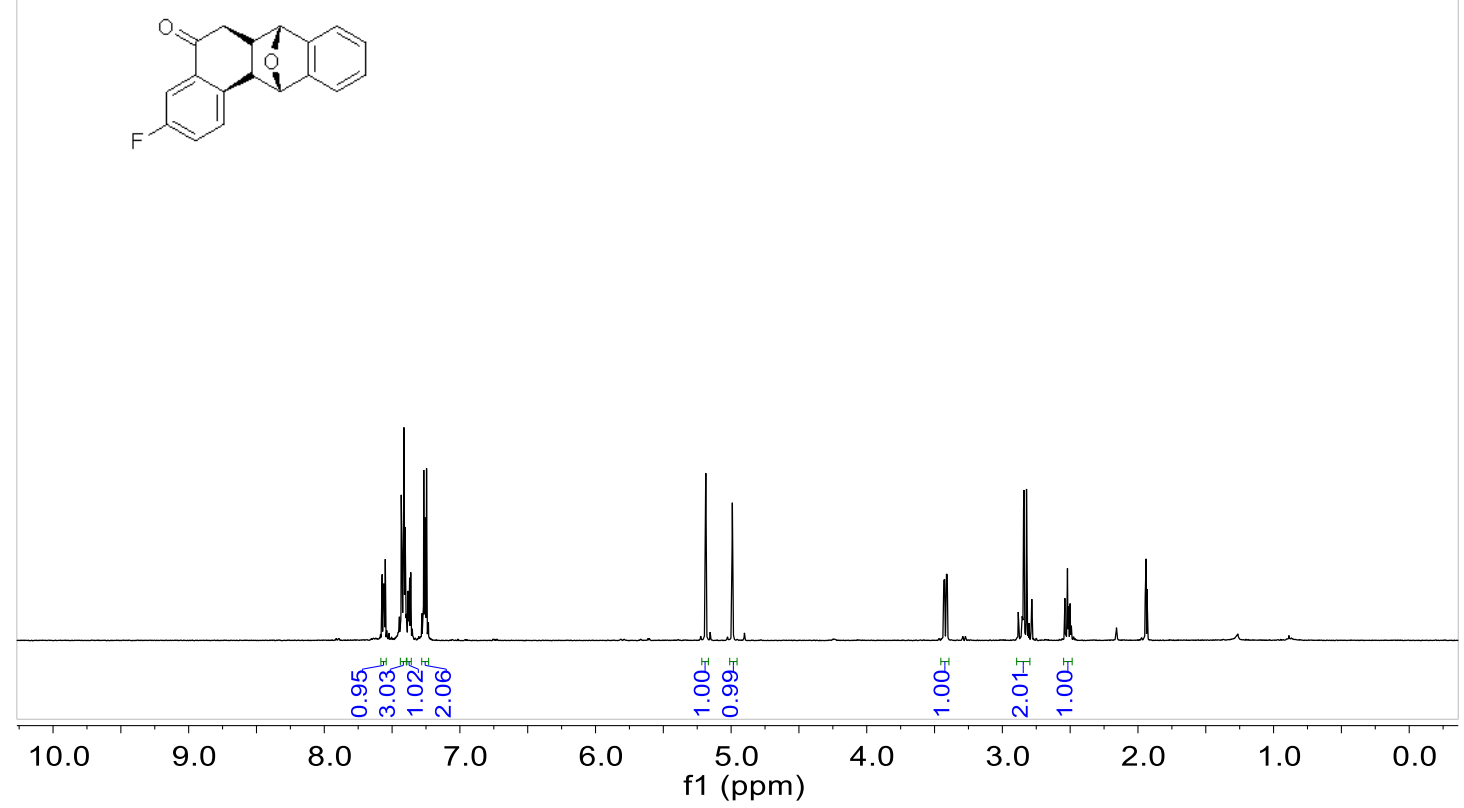

${ }^{1} \mathrm{H}$ NMR (400 MHz, CD $\mathrm{CN}$ ) spectrum of $3 \mathrm{ma}$

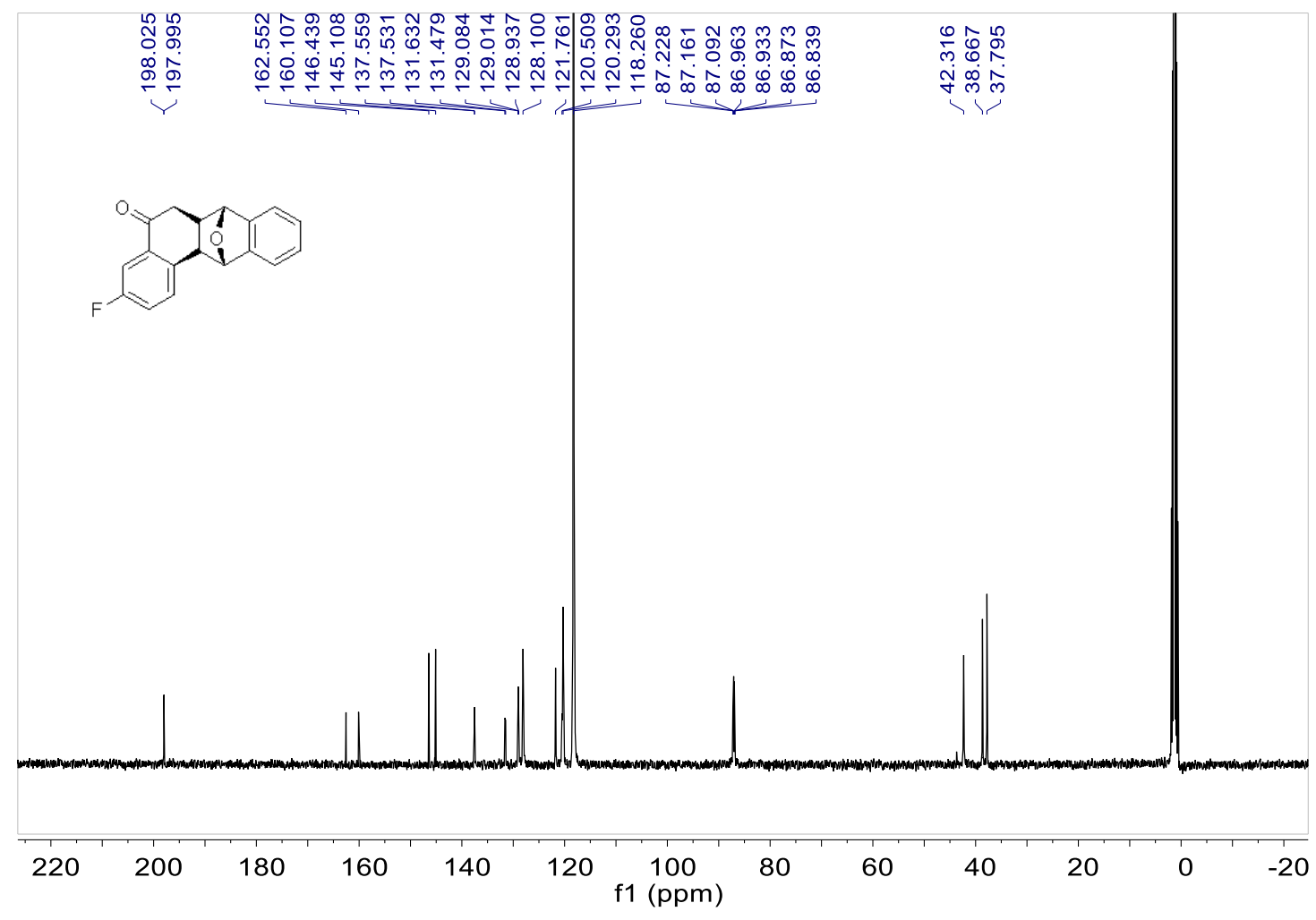

${ }^{13} \mathrm{C}$ NMR (100 MHz, $\left.\mathrm{CD}_{3} \mathrm{CN}\right)$ spectrum of $3 \mathrm{ma}$ 

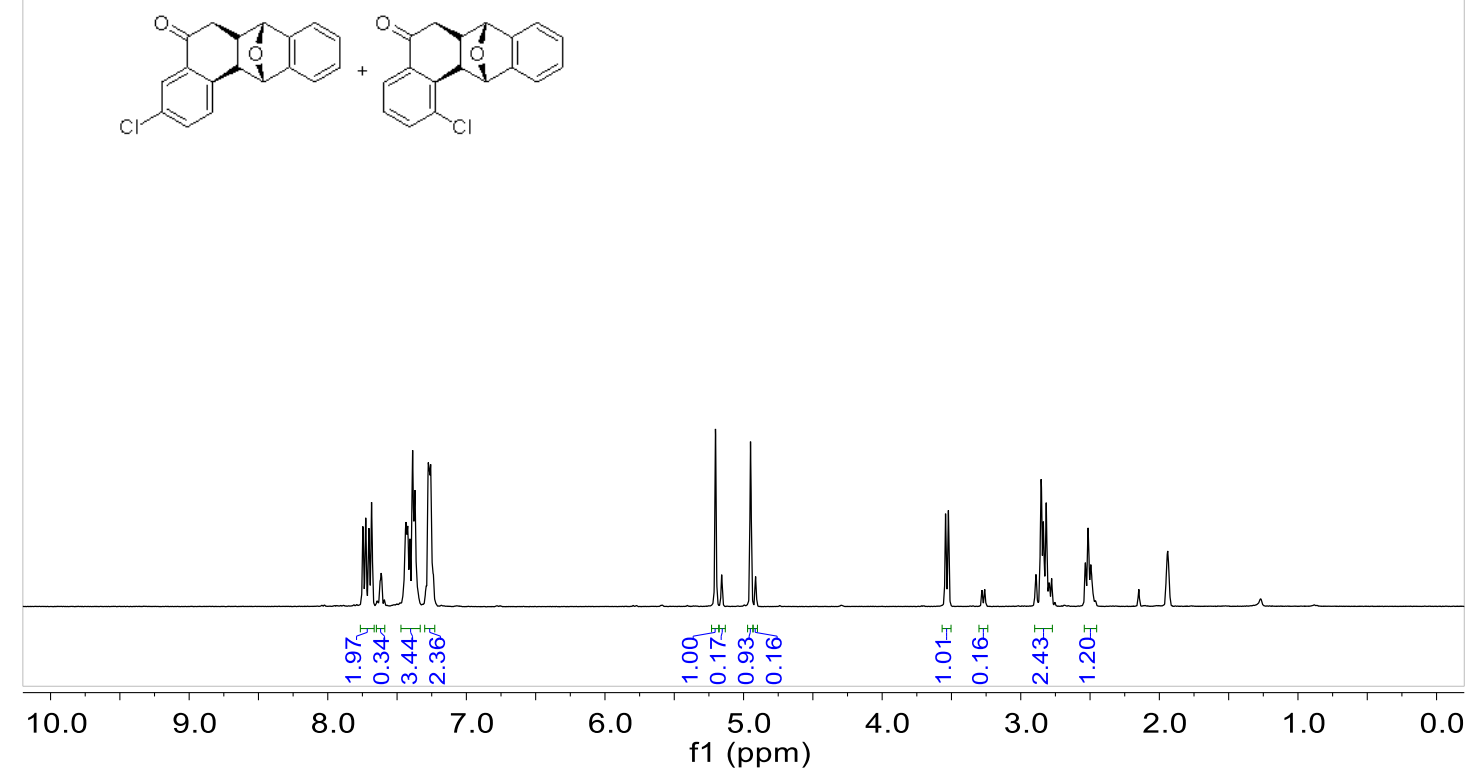

${ }^{1} \mathrm{H}$ NMR (400 MHz, $\left.\mathrm{CD}_{3} \mathrm{CN}\right)$ spectrum of $3 \mathrm{na}$

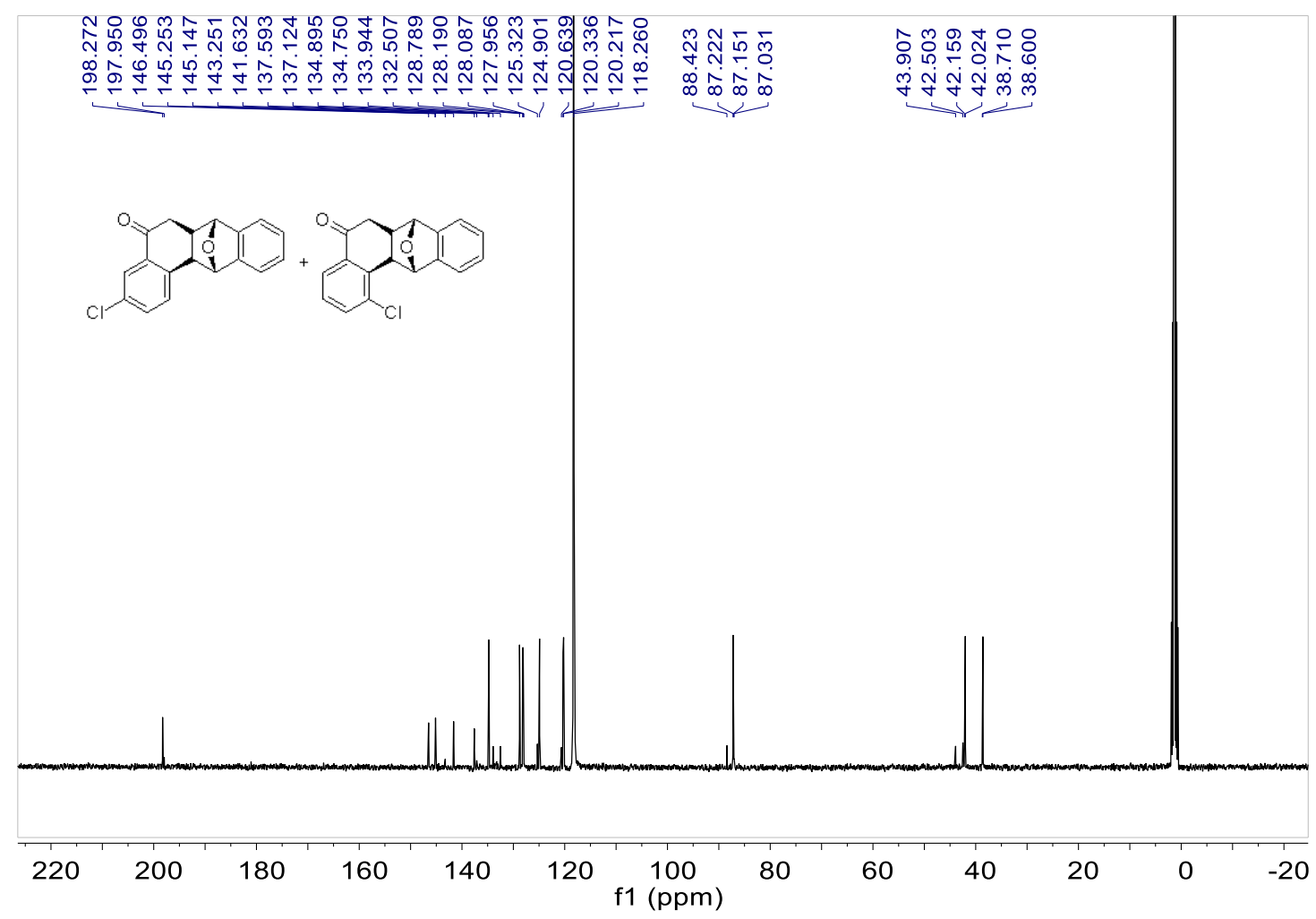

${ }^{13} \mathrm{C}$ NMR (100 MHz, $\mathrm{CD}_{3} \mathrm{CN}$ ) spectrum of 3na 


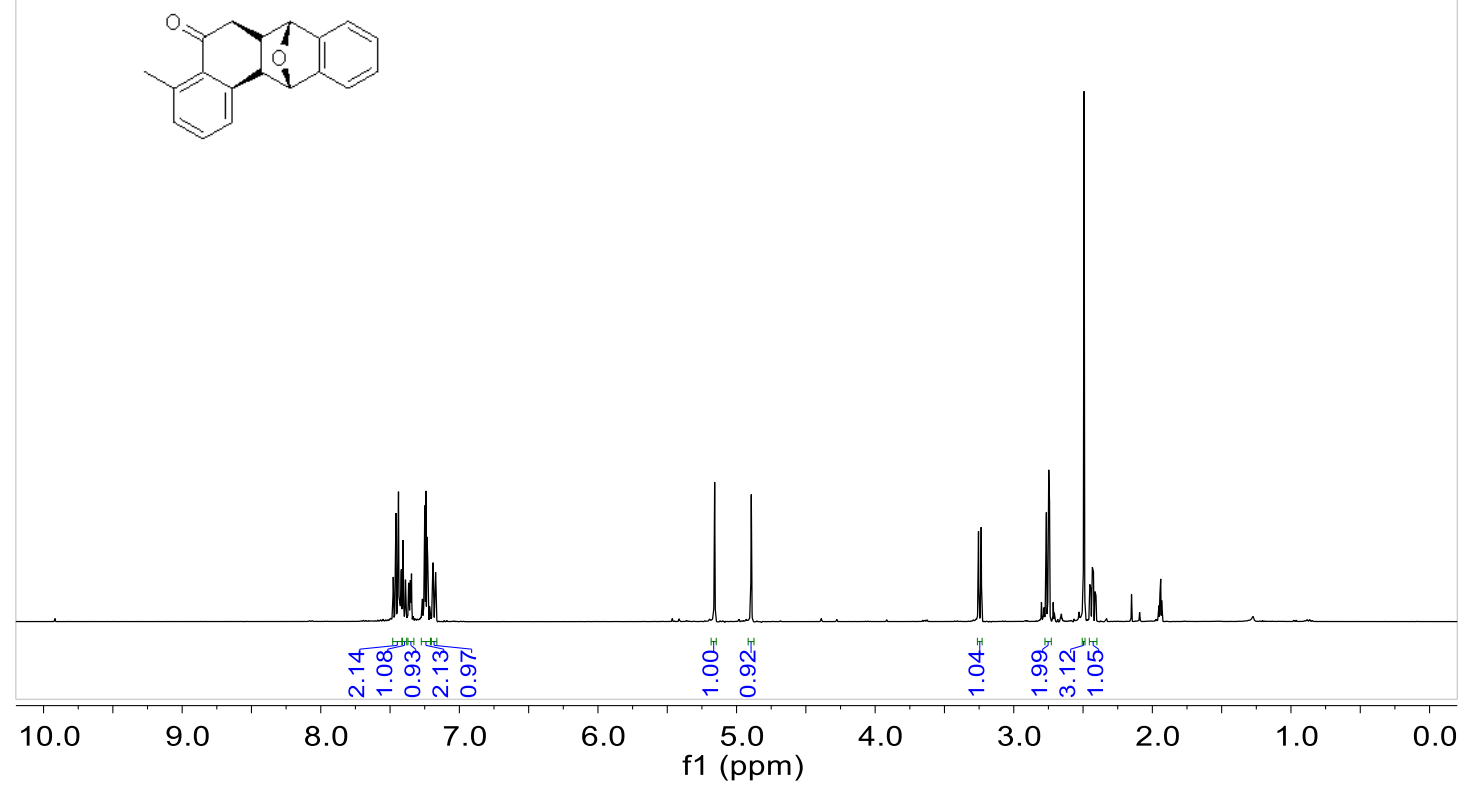

${ }^{1} \mathrm{H}$ NMR (400 MHz, $\mathrm{CD}_{3} \mathrm{CN}$ ) spectrum of $30 a$

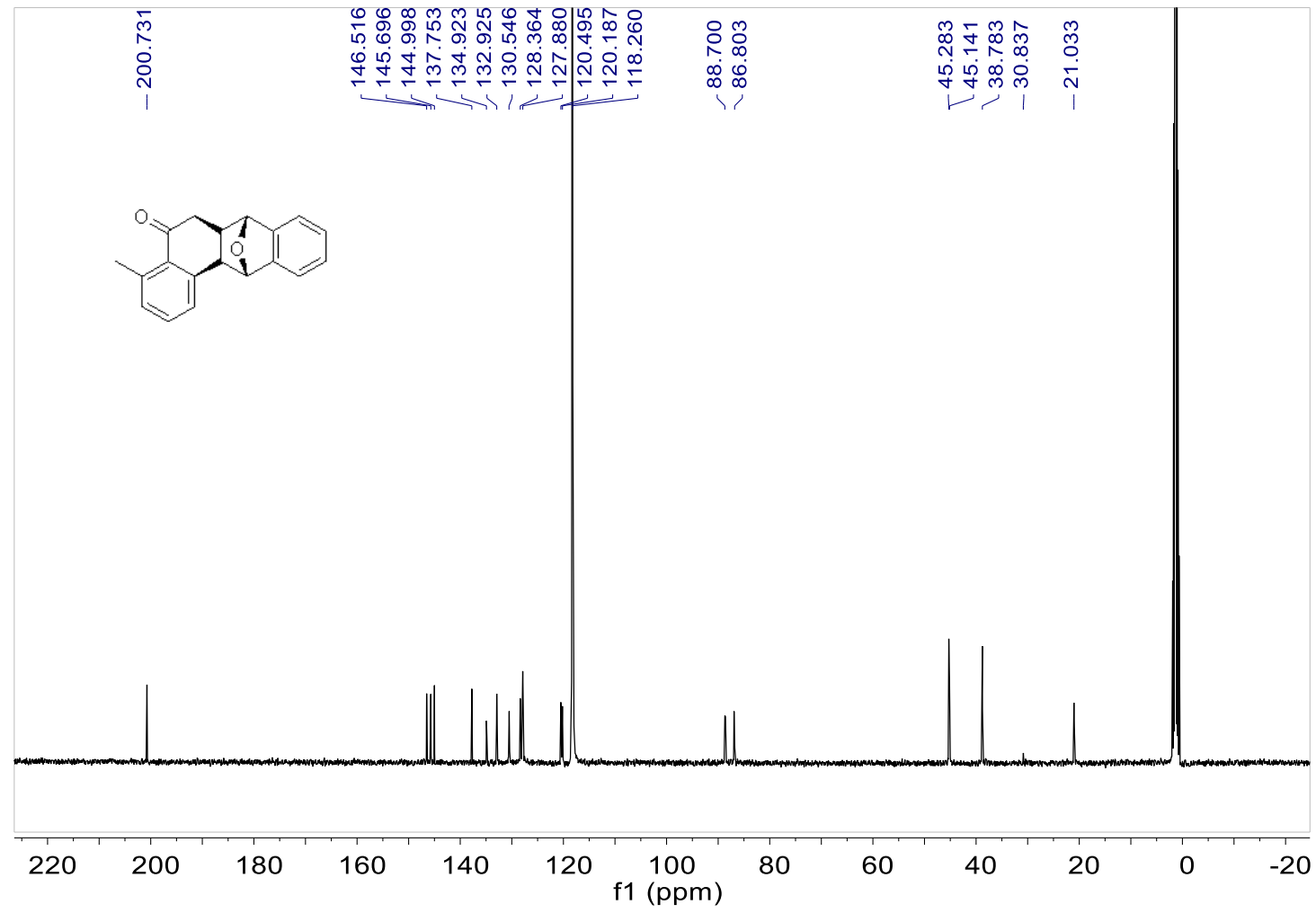

${ }^{13} \mathrm{C}$ NMR (100 MHz, $\mathrm{CD}_{3} \mathrm{CN}$ ) spectrum of $30 a$ 


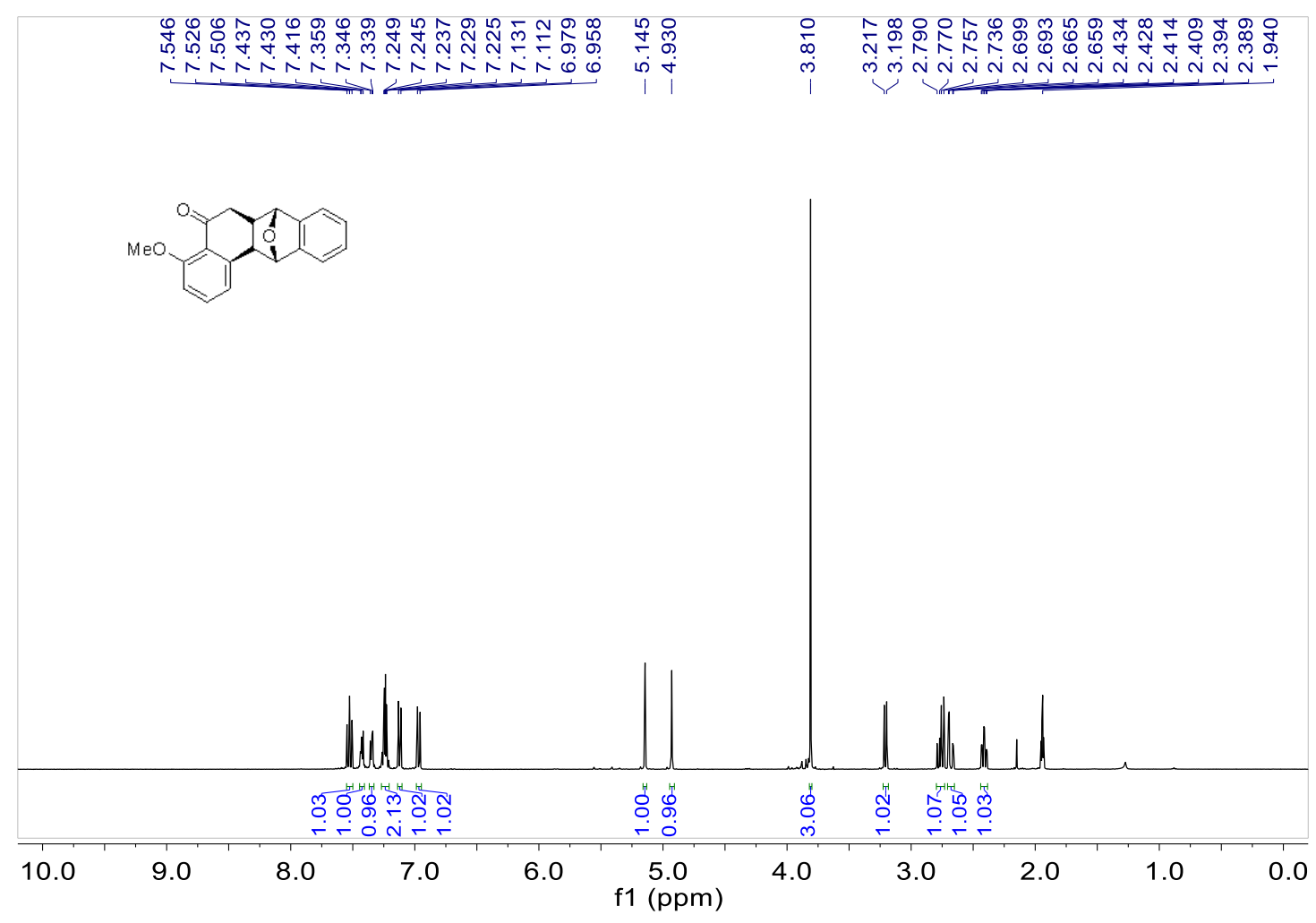

${ }^{1} \mathrm{H}$ NMR (400 MHz, CD $\mathrm{CN}$ ) spectrum of 3 pa

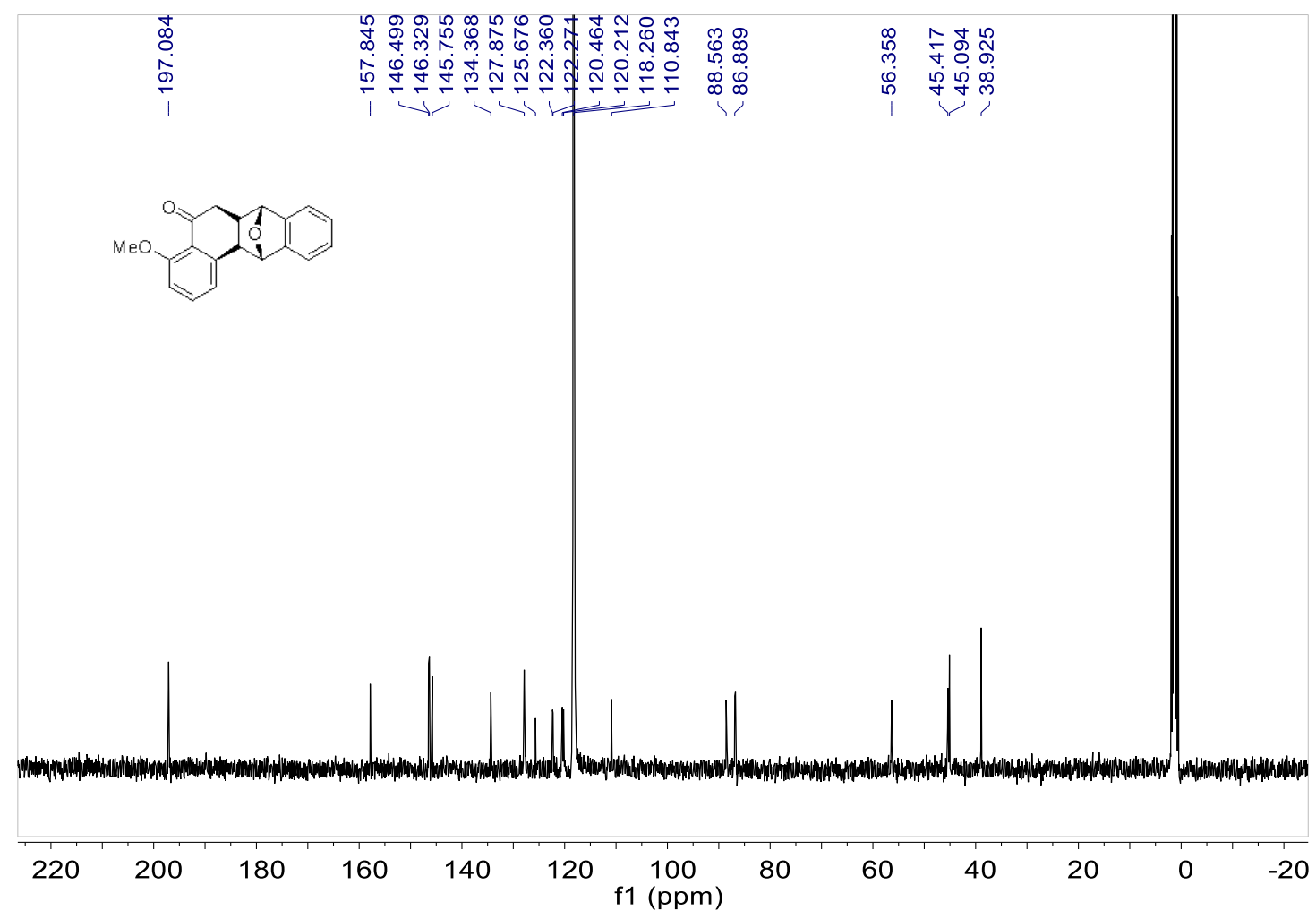

${ }^{13} \mathrm{C}$ NMR (100 MHz, CD $\mathrm{CDN}_{3} \mathrm{CNectrum}$ of $3 \mathrm{pa}$ 

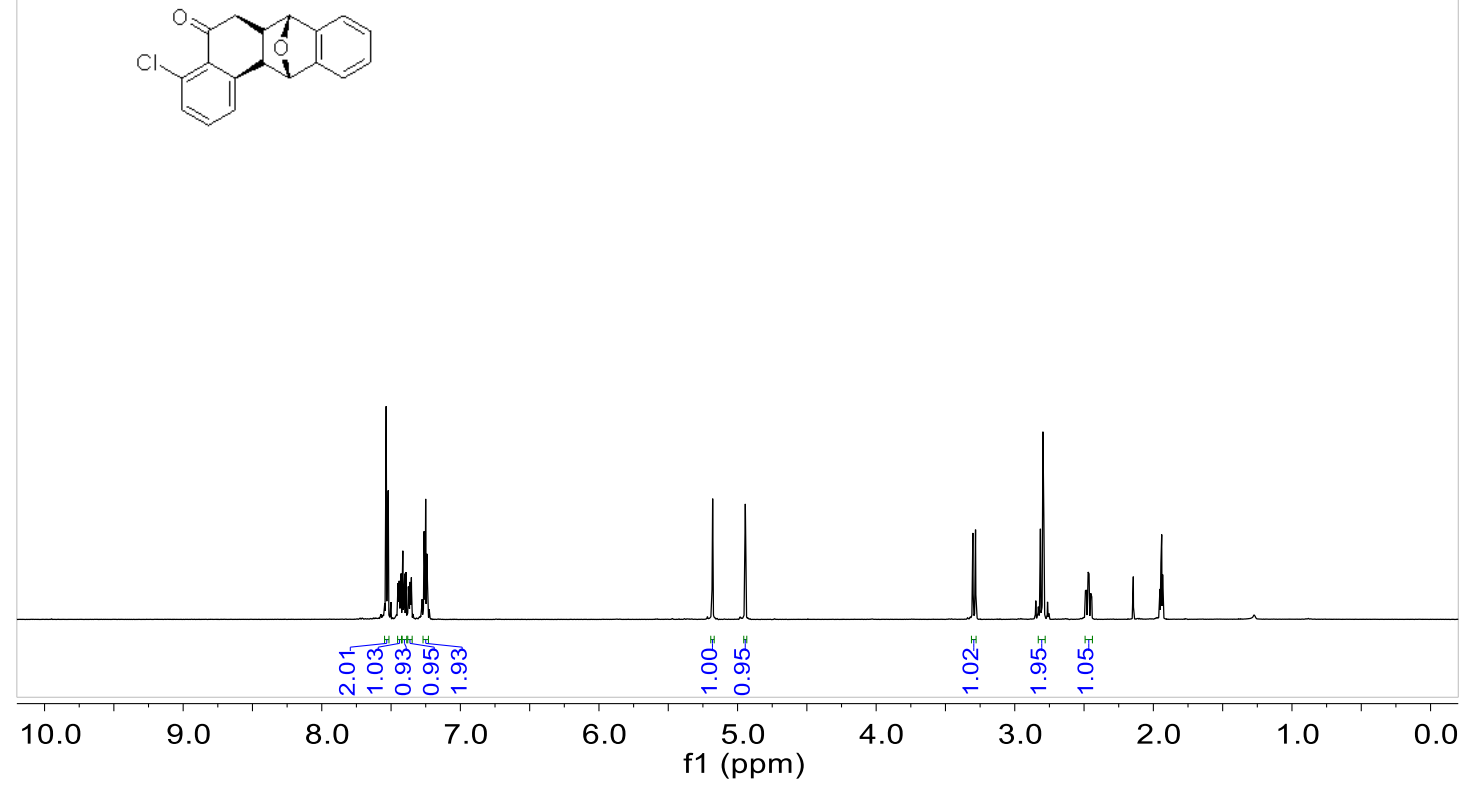

${ }^{1} \mathrm{H}$ NMR (400 MHz, $\mathrm{CD}_{3} \mathrm{CN}$ ) spectrum of 3qa

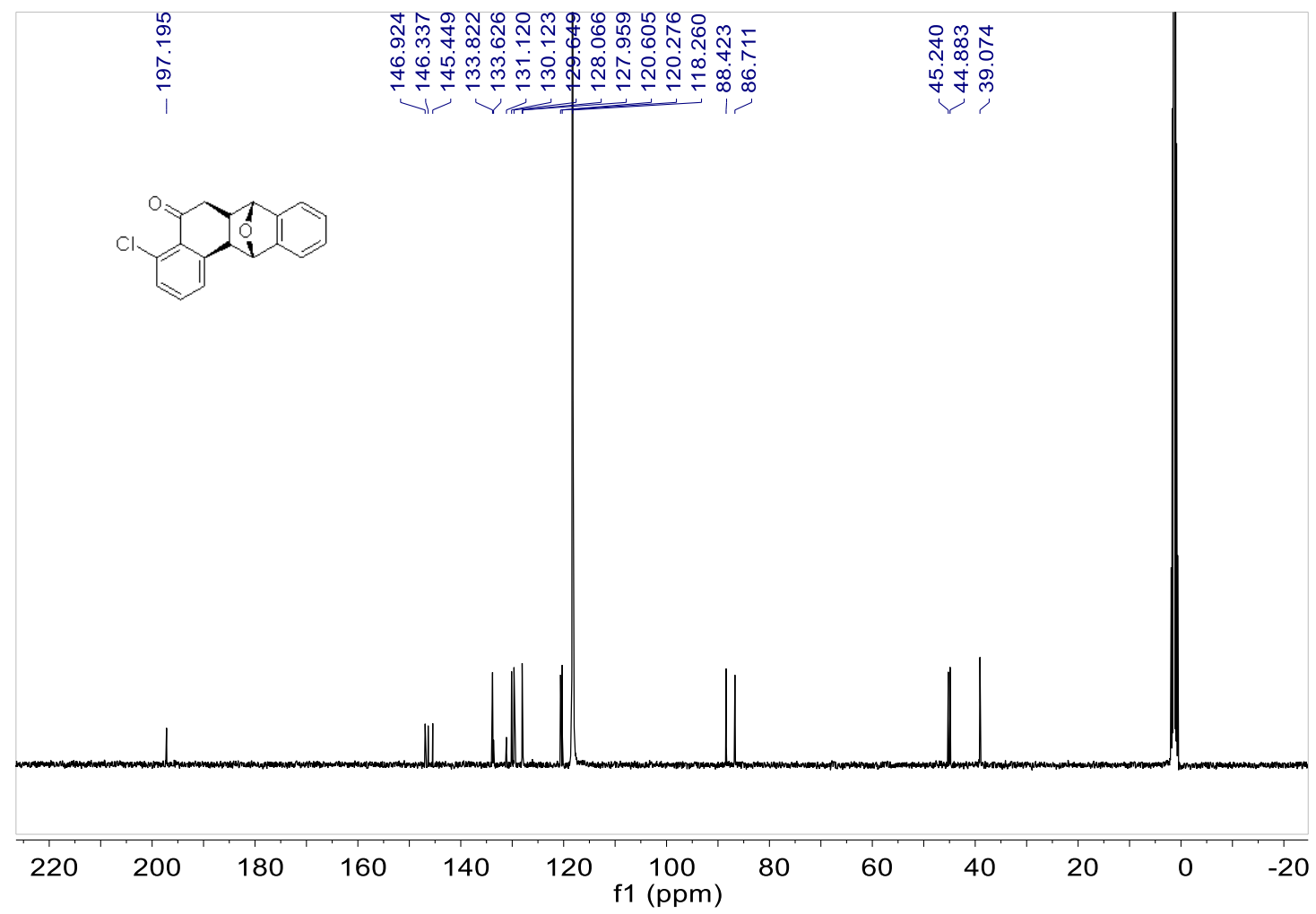

${ }^{13} \mathrm{C}$ NMR (100 MHz, $\mathrm{CD}_{3} \mathrm{CN}$ ) spectrum of 3qa 


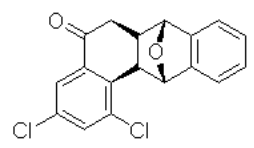

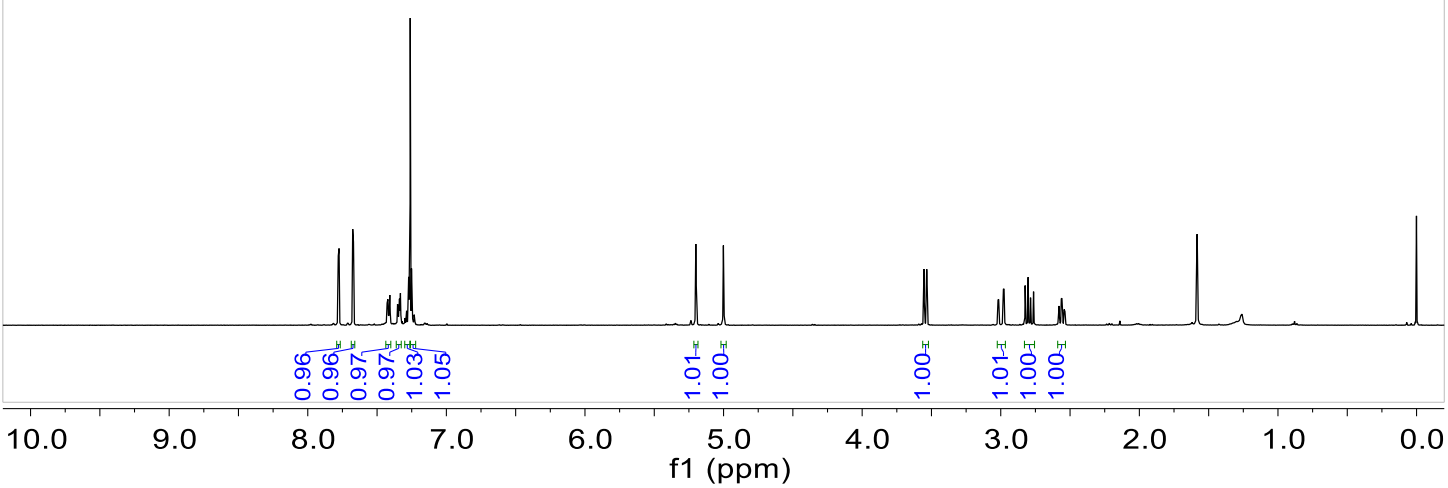

${ }^{1} \mathrm{H}$ NMR (400 MHz, $\mathrm{CDCl}_{3}$ ) spectrum of 3ra

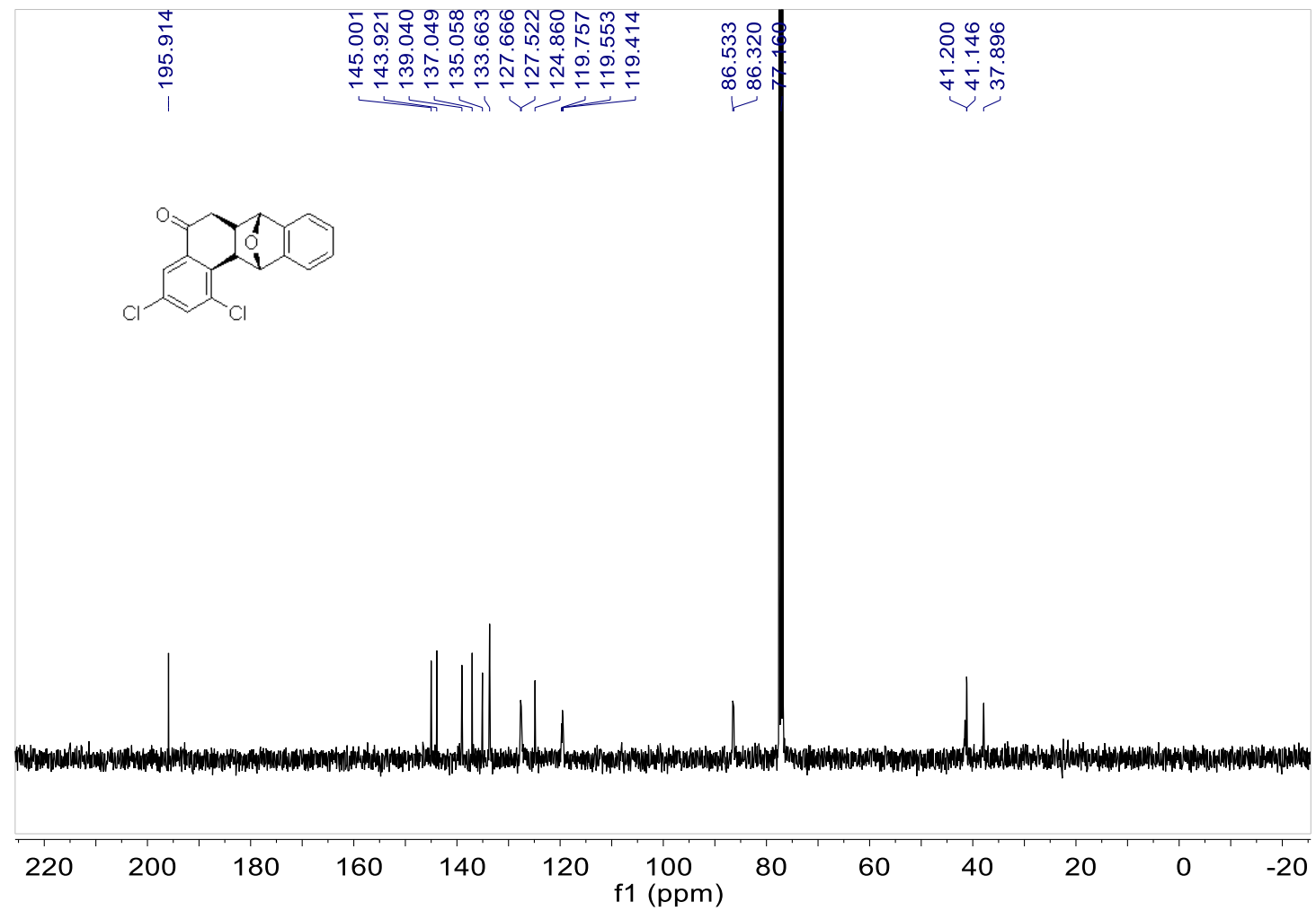

${ }^{13} \mathrm{C}$ NMR (100 MHz, $\left.\mathrm{CDCl}_{3}\right)$ spectrum of 3ra 


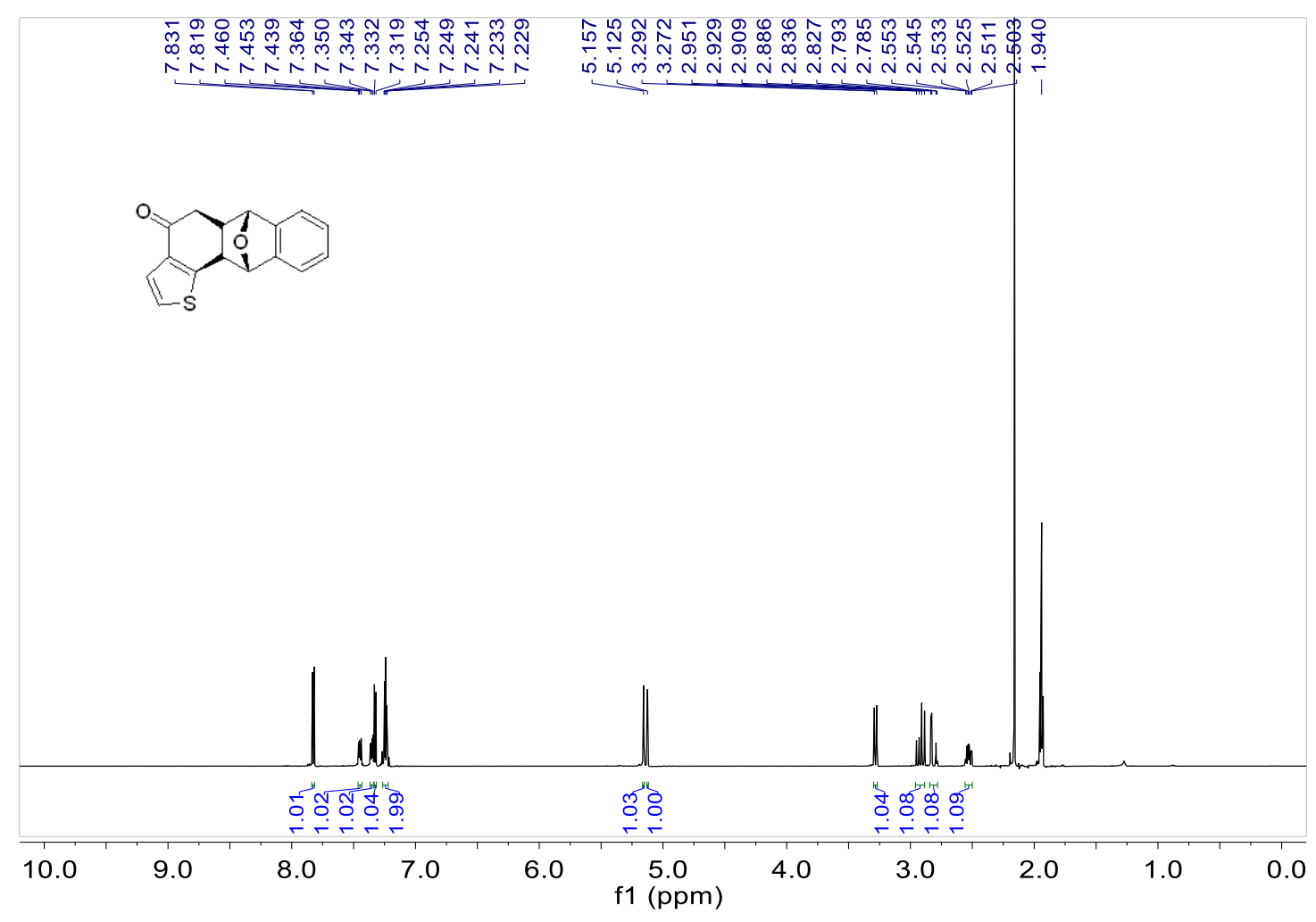

${ }^{1} \mathrm{H}$ NMR (400 MHz, CD $\mathrm{CDN}_{3}$ ) spectrum of $3 \mathrm{sa}$

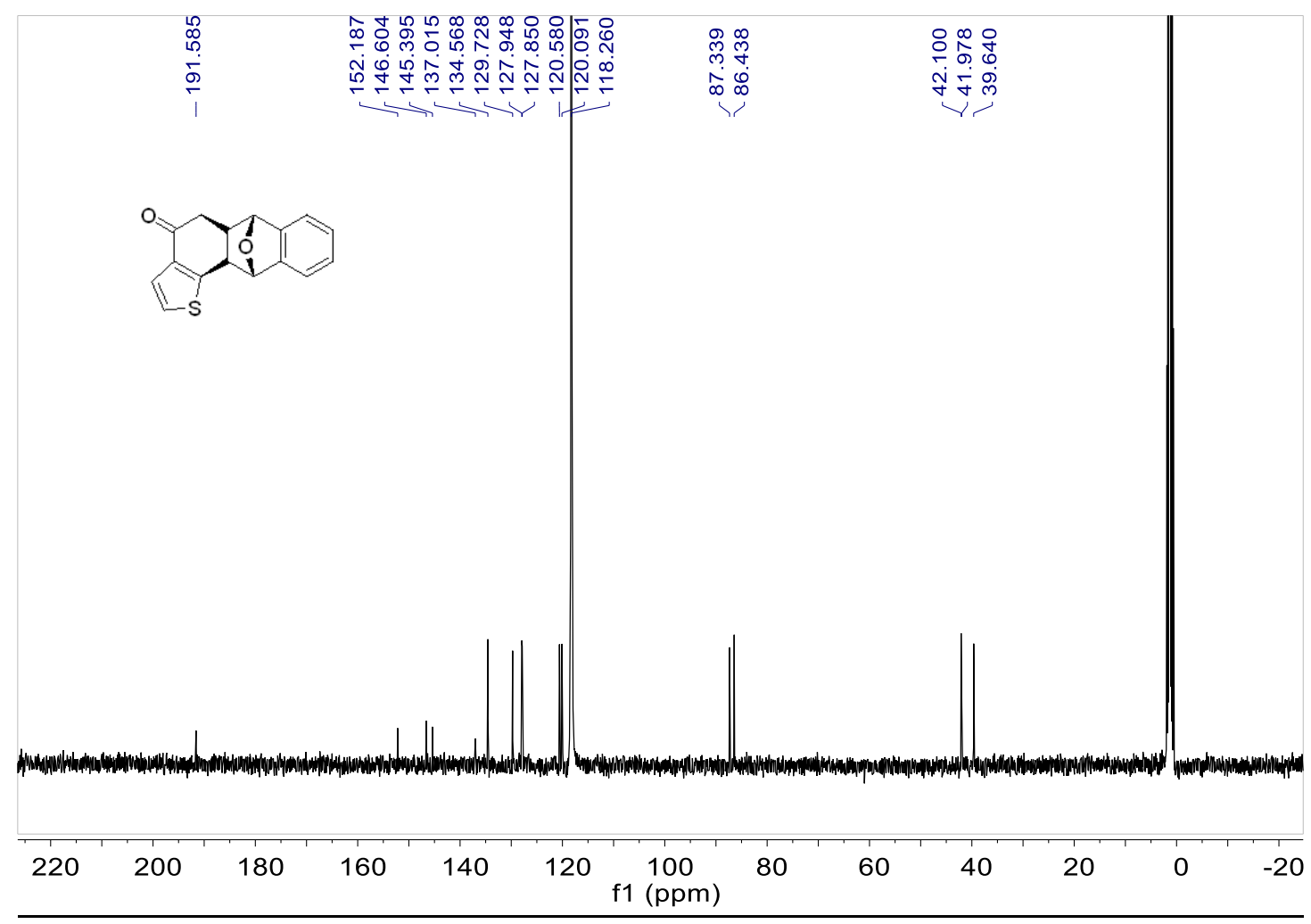

${ }^{13} \mathrm{C}$ NMR (100 MHz, $\left.\mathrm{CD}_{3} \mathrm{CN}\right)$ spectrum of 3sa 

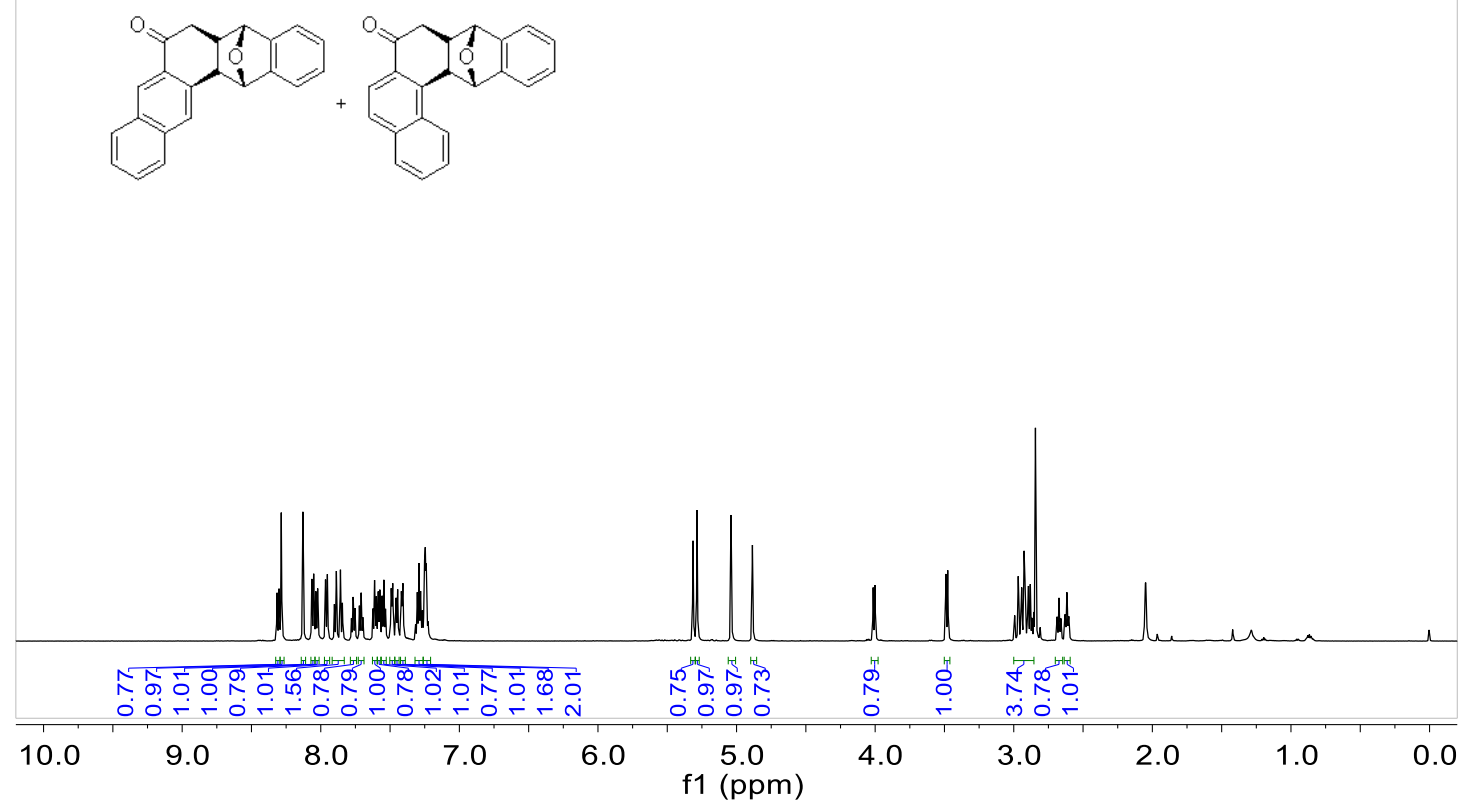

${ }^{1} \mathrm{H}$ NMR $\left(600 \mathrm{MHz},\left(\mathrm{CD}_{3}\right)_{2} \mathrm{CO}\right)$ spectrum of 3 ta

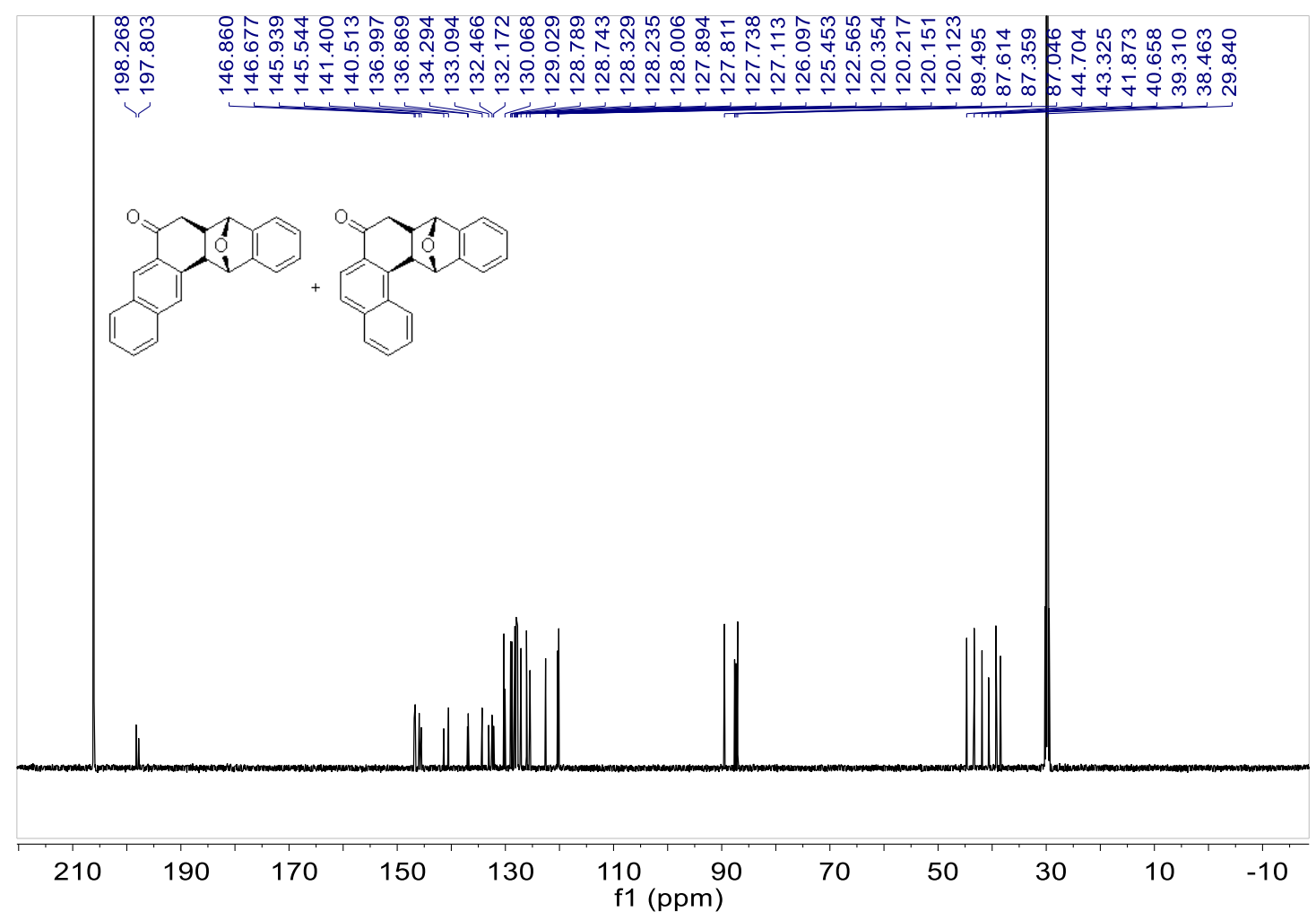

${ }^{13} \mathrm{C}$ NMR $\left(150 \mathrm{MHz},\left(\mathrm{CD}_{3}\right)_{2} \mathrm{CO}\right)$ spectrum of 3 ta 


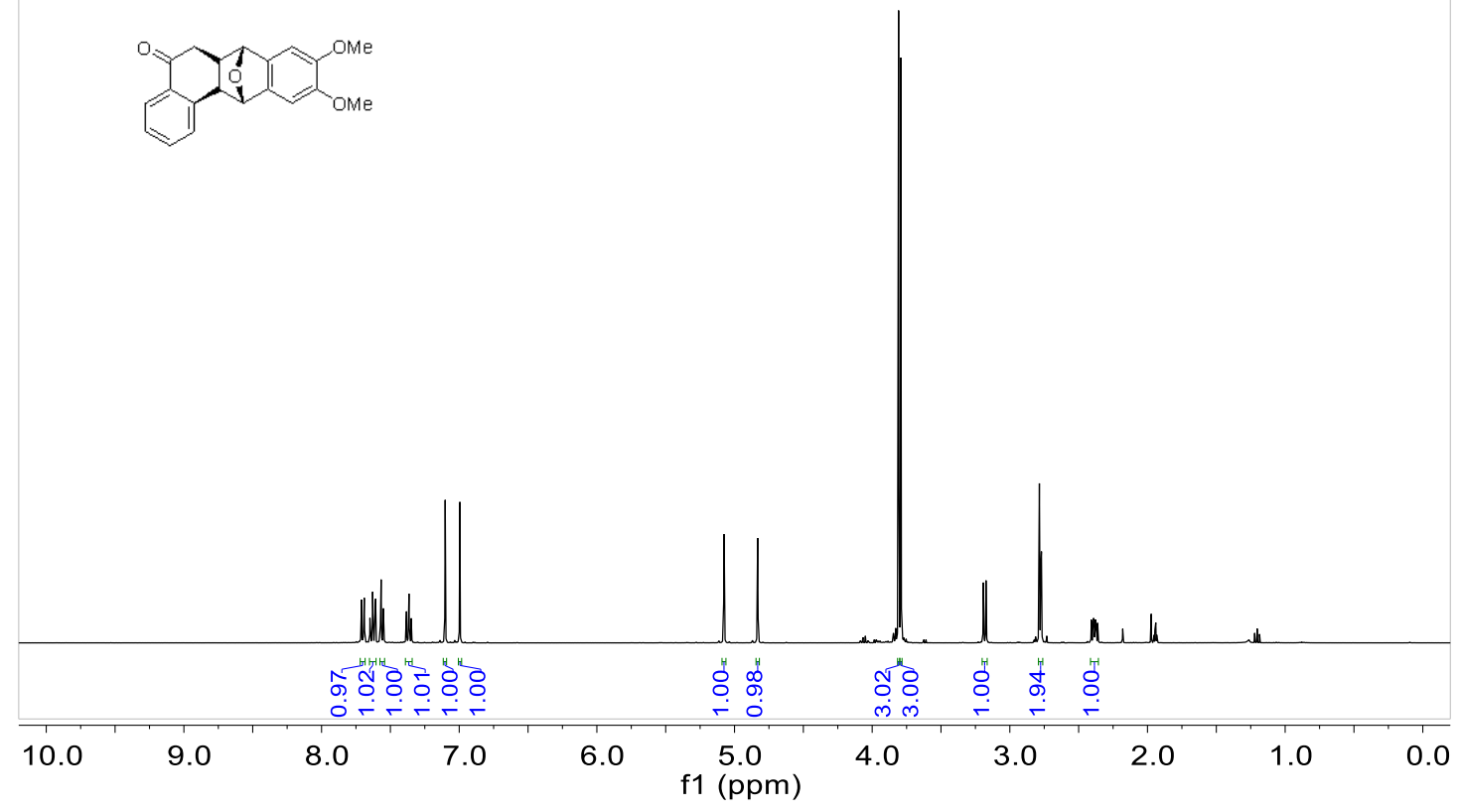

${ }^{1} \mathrm{H}$ NMR (400 MHz, $\mathrm{CD}_{3} \mathrm{CN}$ ) spectrum of 3ab

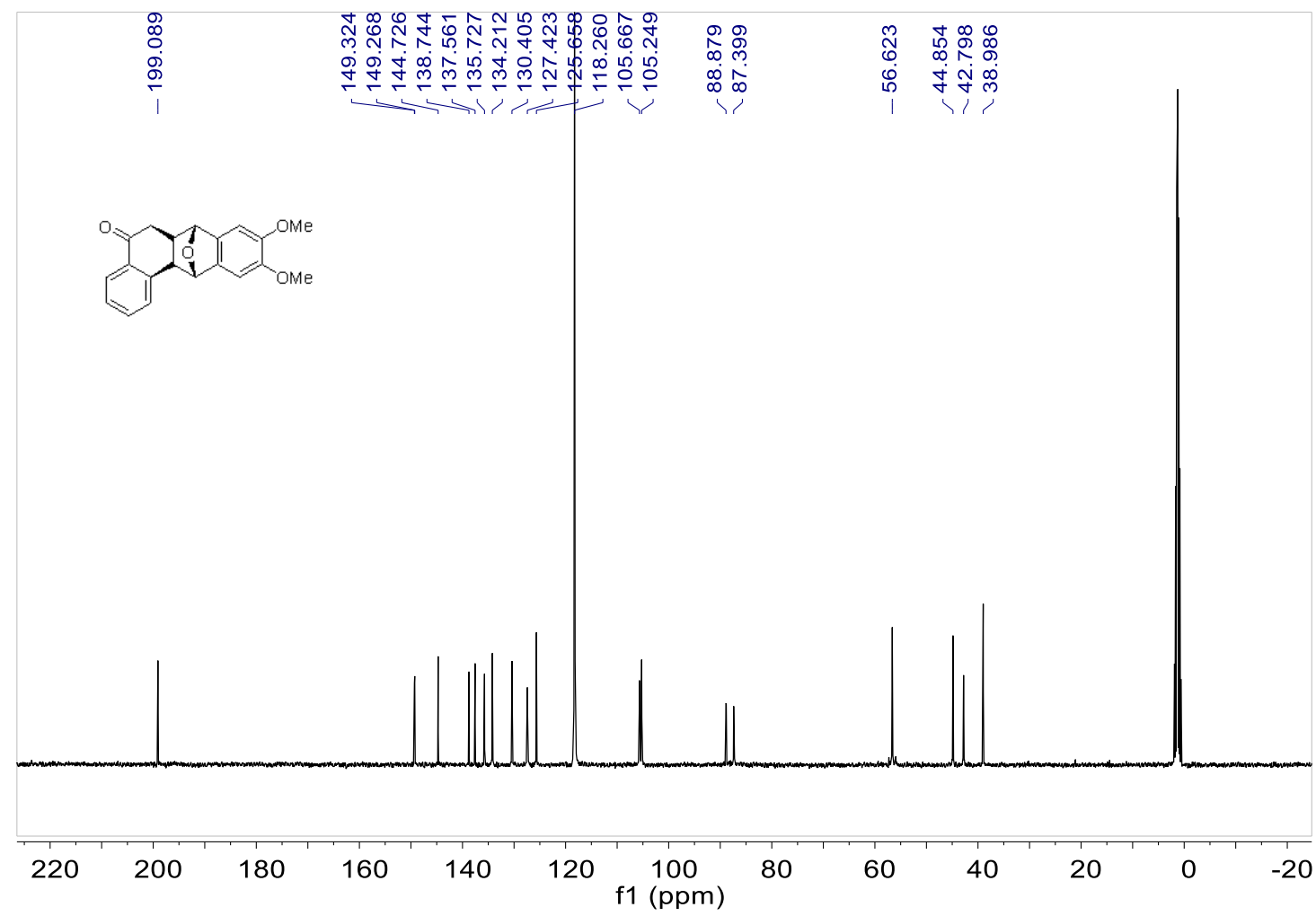

${ }^{13} \mathrm{C}$ NMR (100 MHz, $\mathrm{CD}_{3} \mathrm{CN}$ ) spectrum of 3ab 


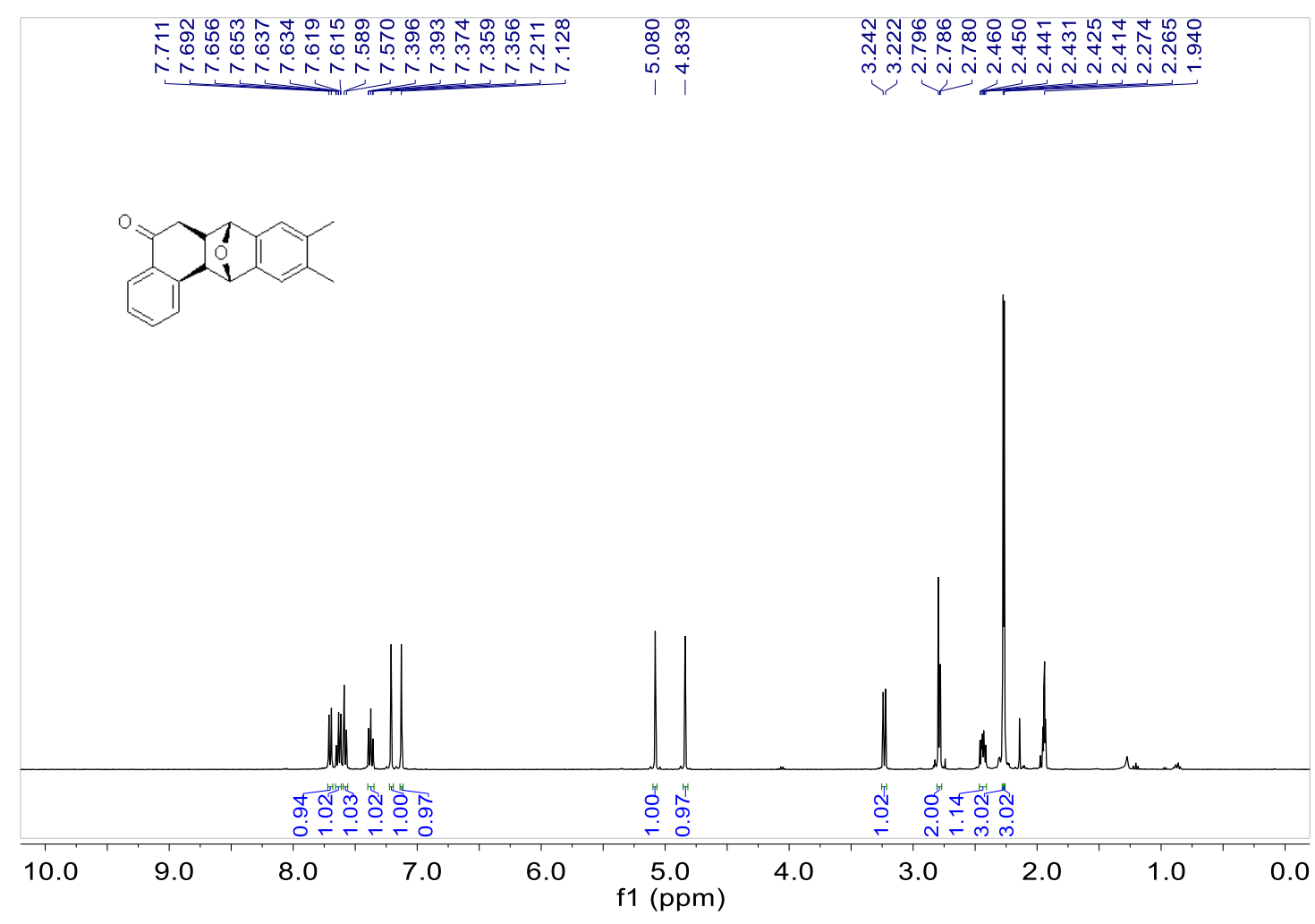

${ }^{1} \mathrm{H}$ NMR (400 MHz, $\mathrm{CD}_{3} \mathrm{CN}$ ) spectrum of $3 \mathrm{ac}$

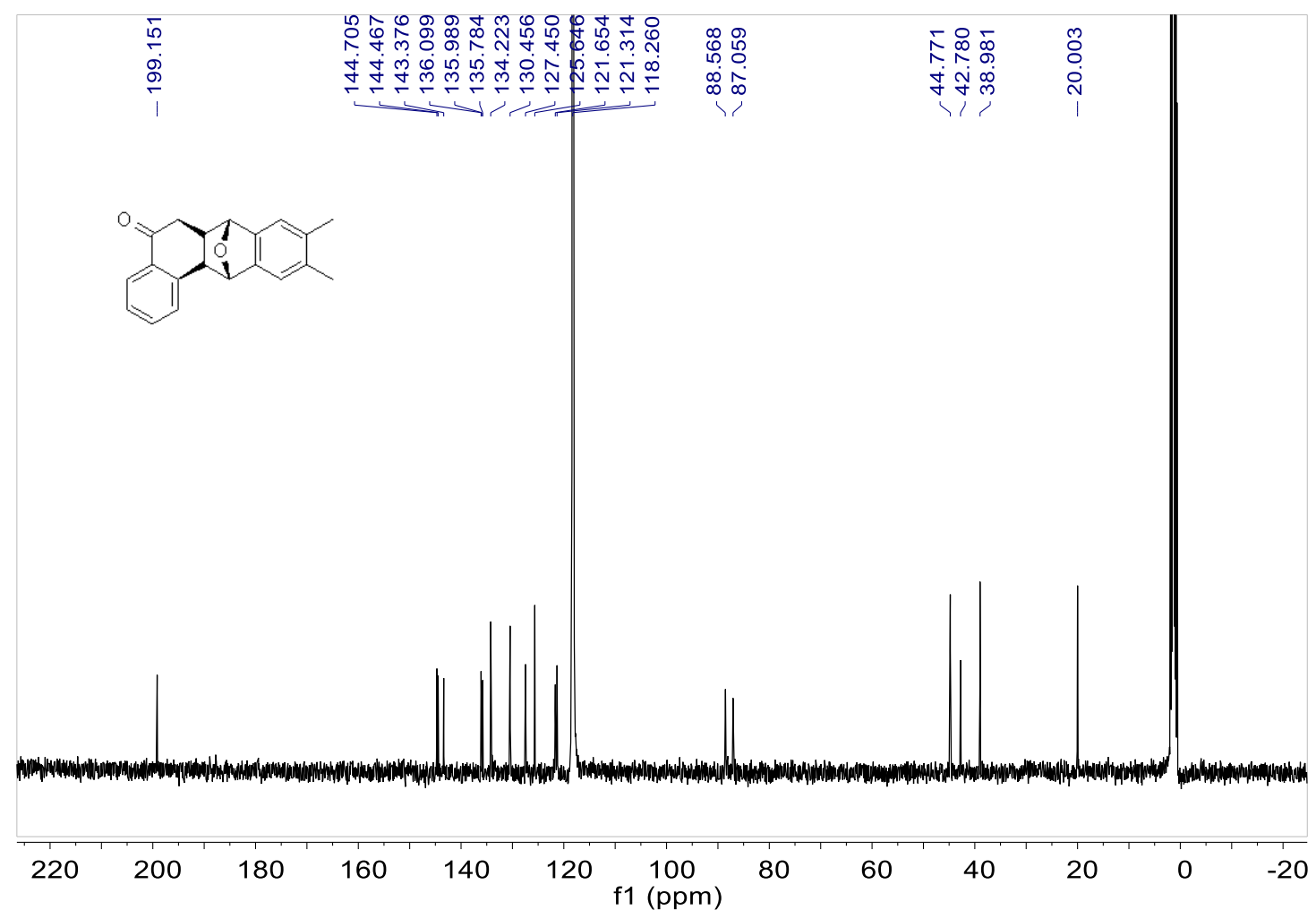

${ }^{13} \mathrm{C}$ NMR (100 MHz, $\left.\mathrm{CD}_{3} \mathrm{CN}\right)$ spectrum of $3 \mathrm{ac}$ 
<smiles>O=C1CC2OC(c3ccccc31)c1cc3c(cc12)OCO3</smiles>

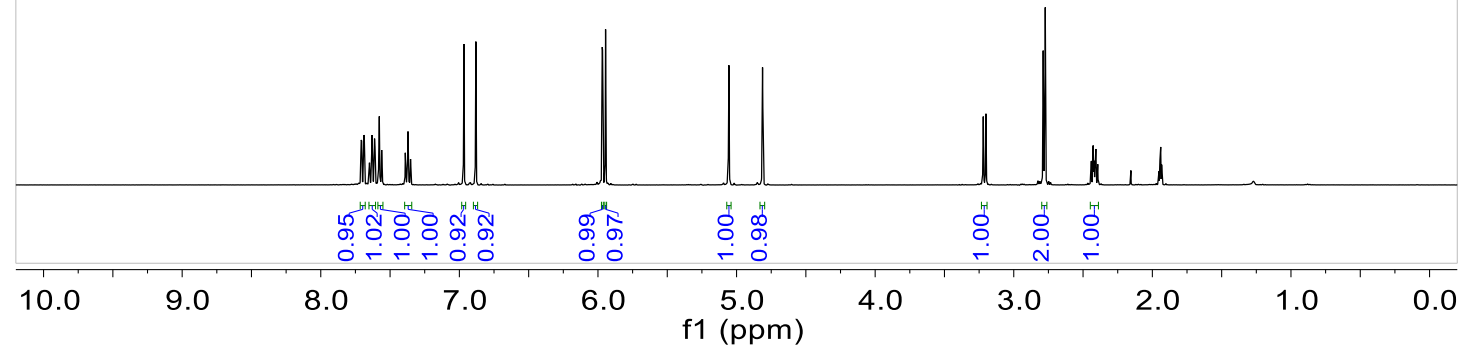

${ }^{1} \mathrm{H}$ NMR (400 MHz, $\left.\mathrm{CD}_{3} \mathrm{CN}\right)$ spectrum of $3 \mathrm{ad}$

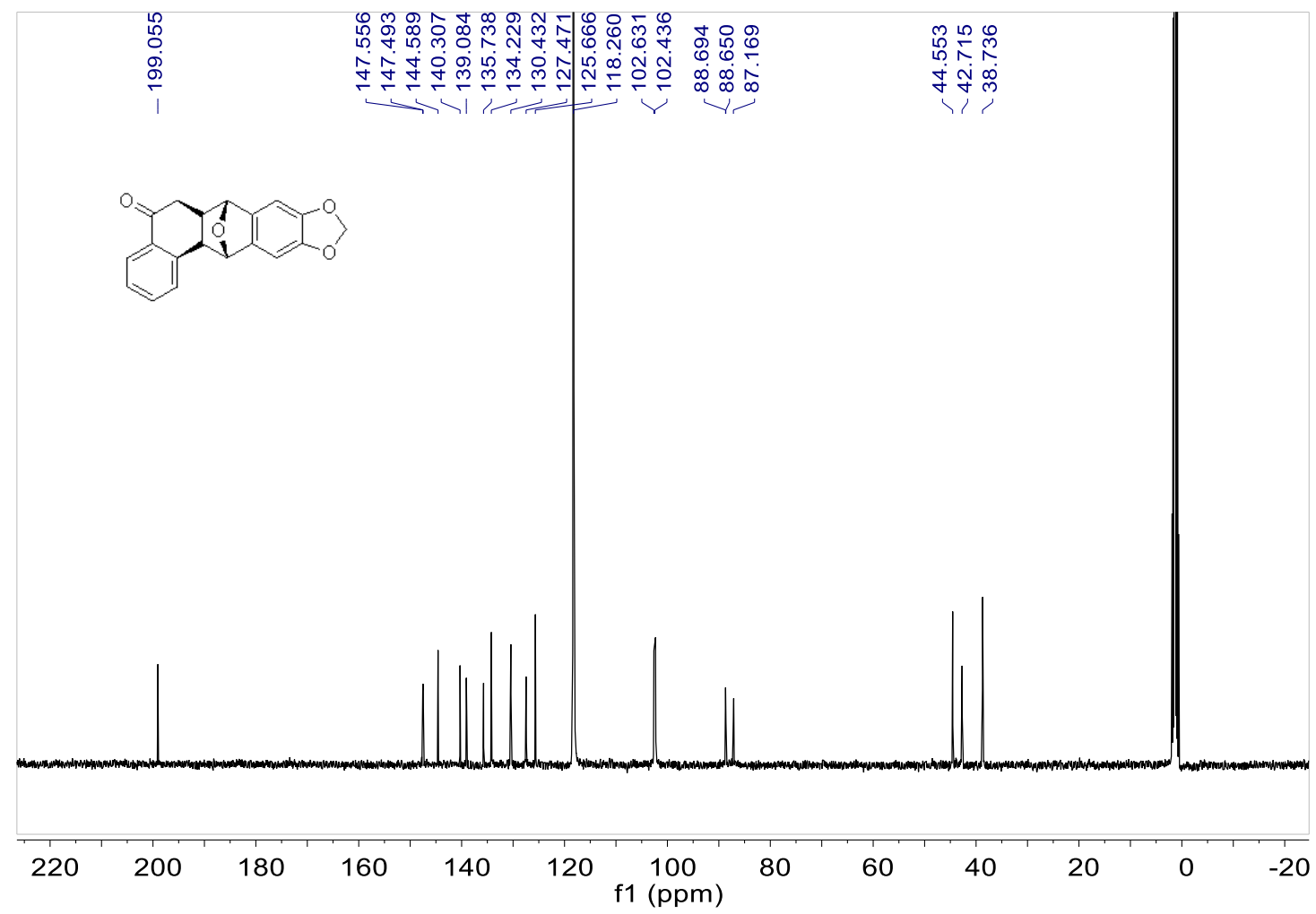

${ }^{13} \mathrm{C}$ NMR (100 MHz, CD $\left.\mathrm{CN}\right)$ spectrum of $3 \mathrm{ad}$ 


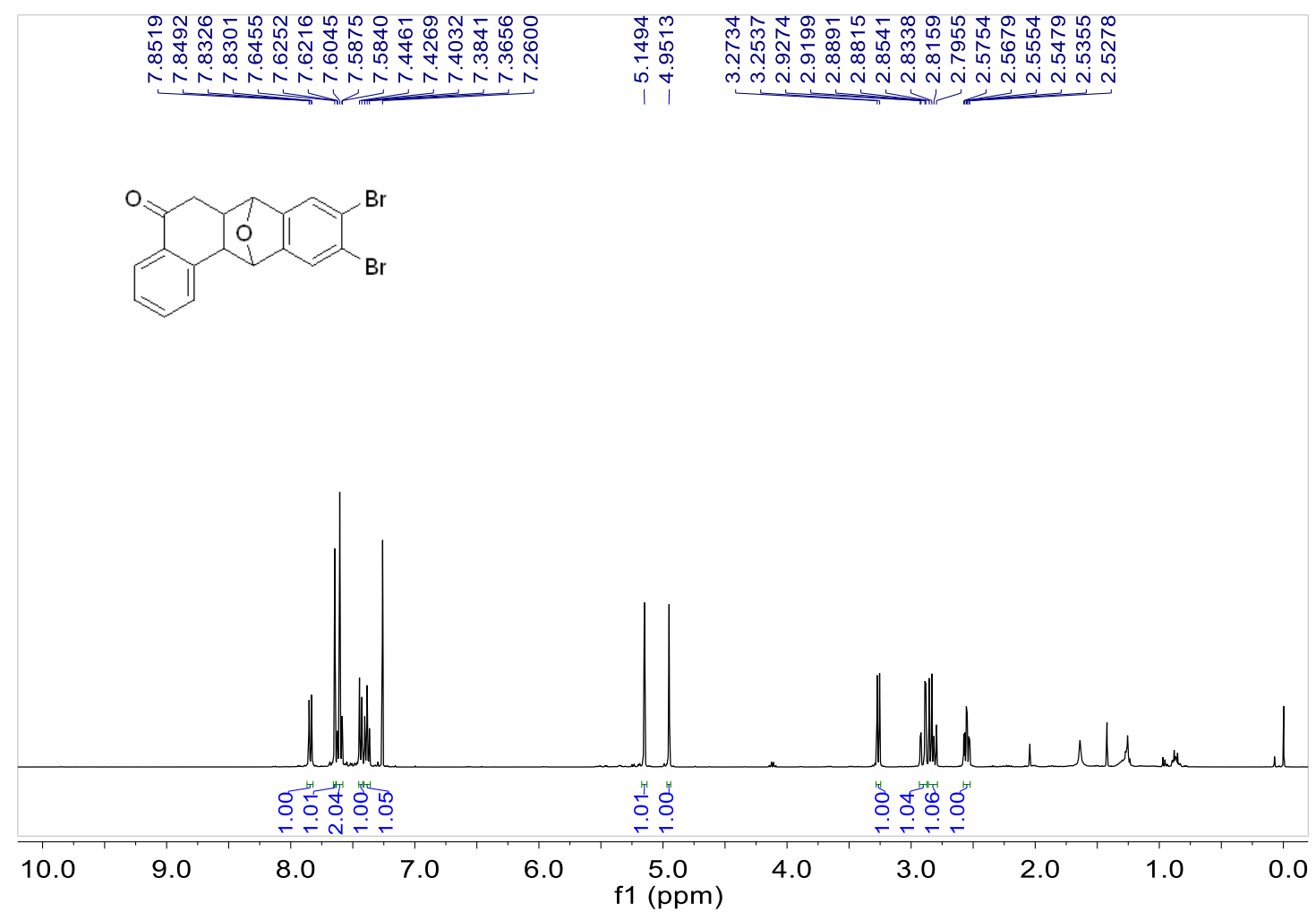

${ }^{1} \mathrm{H}$ NMR (400 MHz, $\mathrm{CDCl}_{3}$ ) spectrum of 3ae

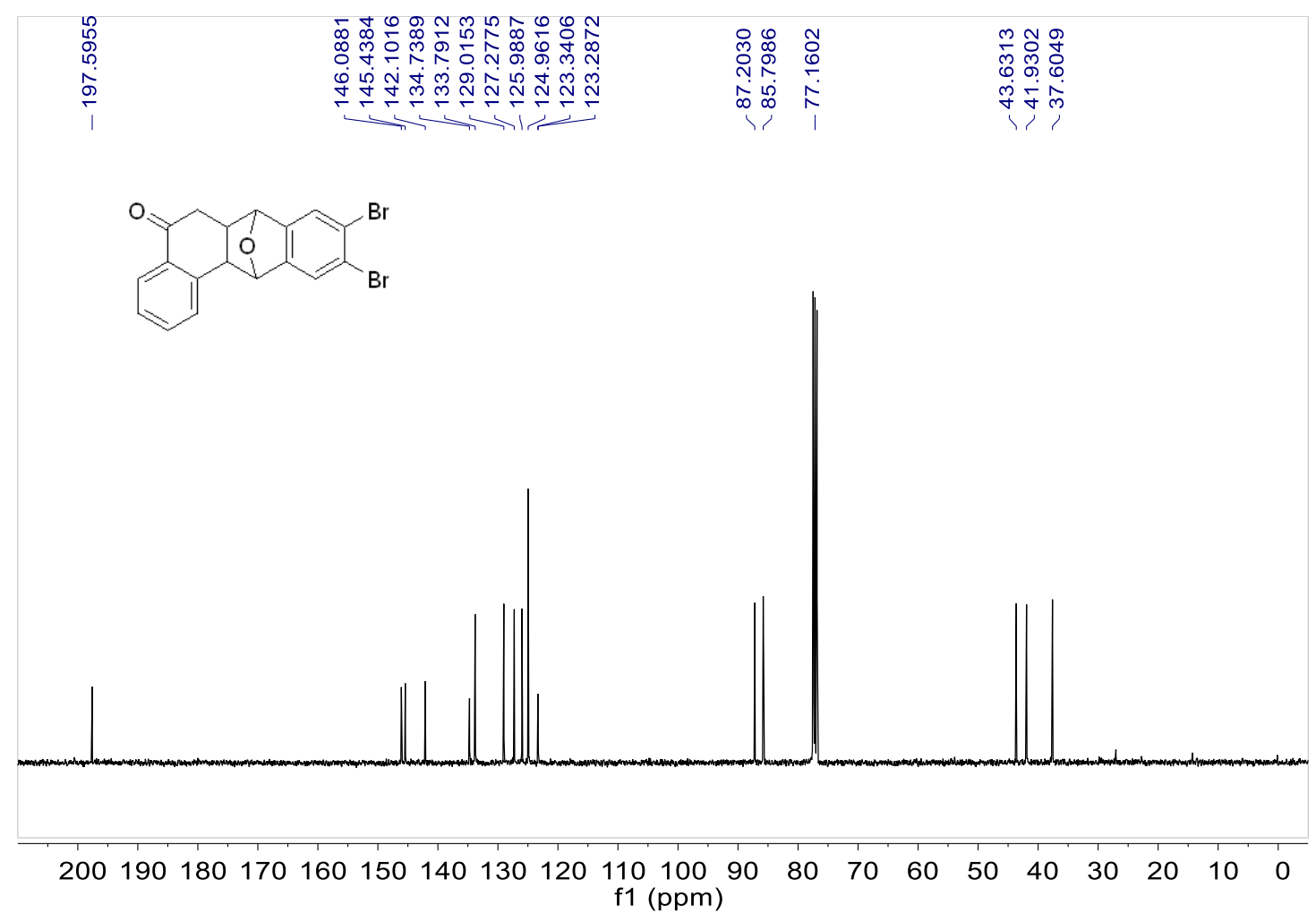

${ }^{13} \mathrm{C}$ NMR (100 MHz, $\left.\mathrm{CDCl}_{3}\right)$ spectrum of $3 \mathrm{ae}$ 

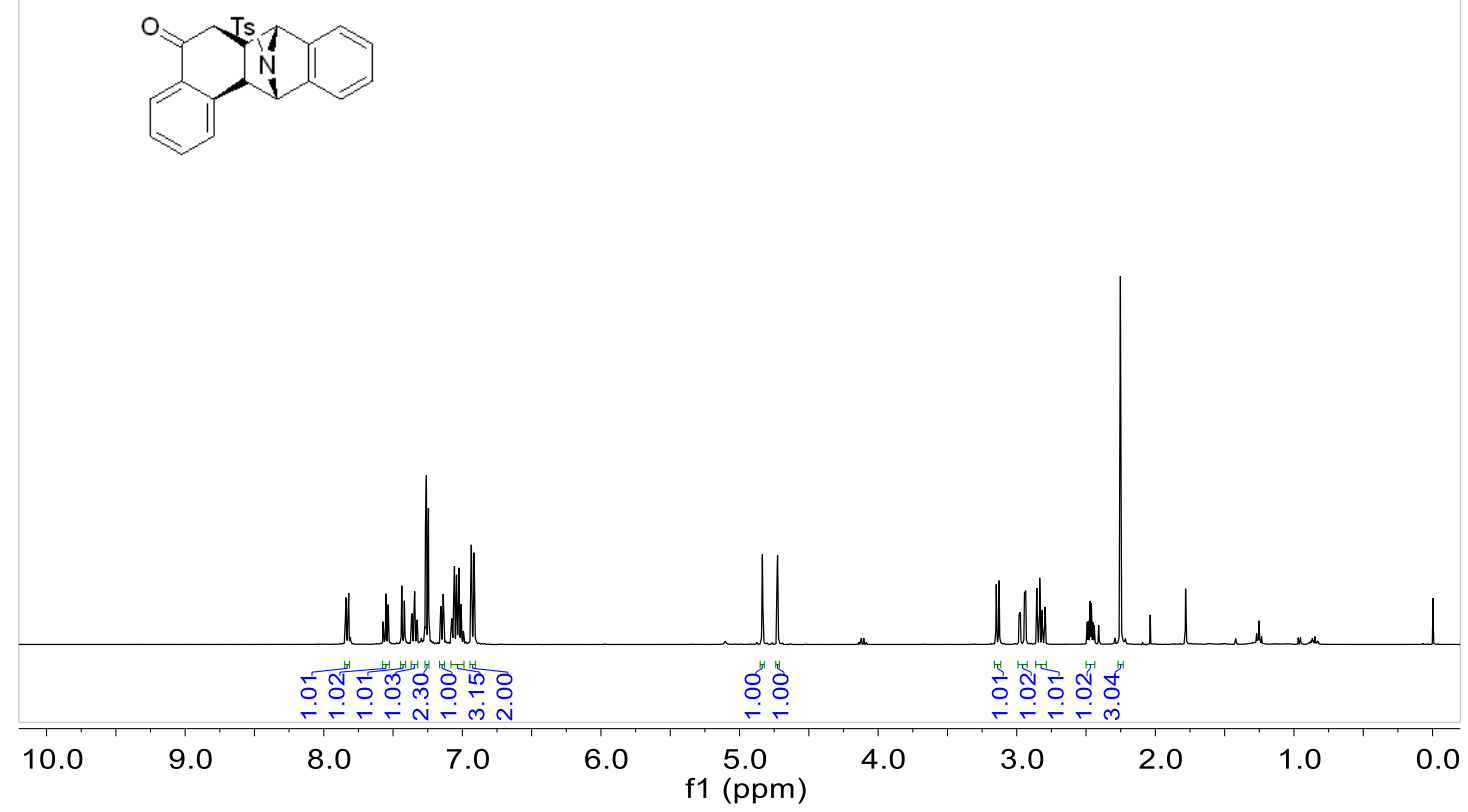

${ }^{1} \mathrm{H}$ NMR (400 MHz, $\left.\mathrm{CDCl}_{3}\right)$ spectrum of $5 \mathrm{aa}$

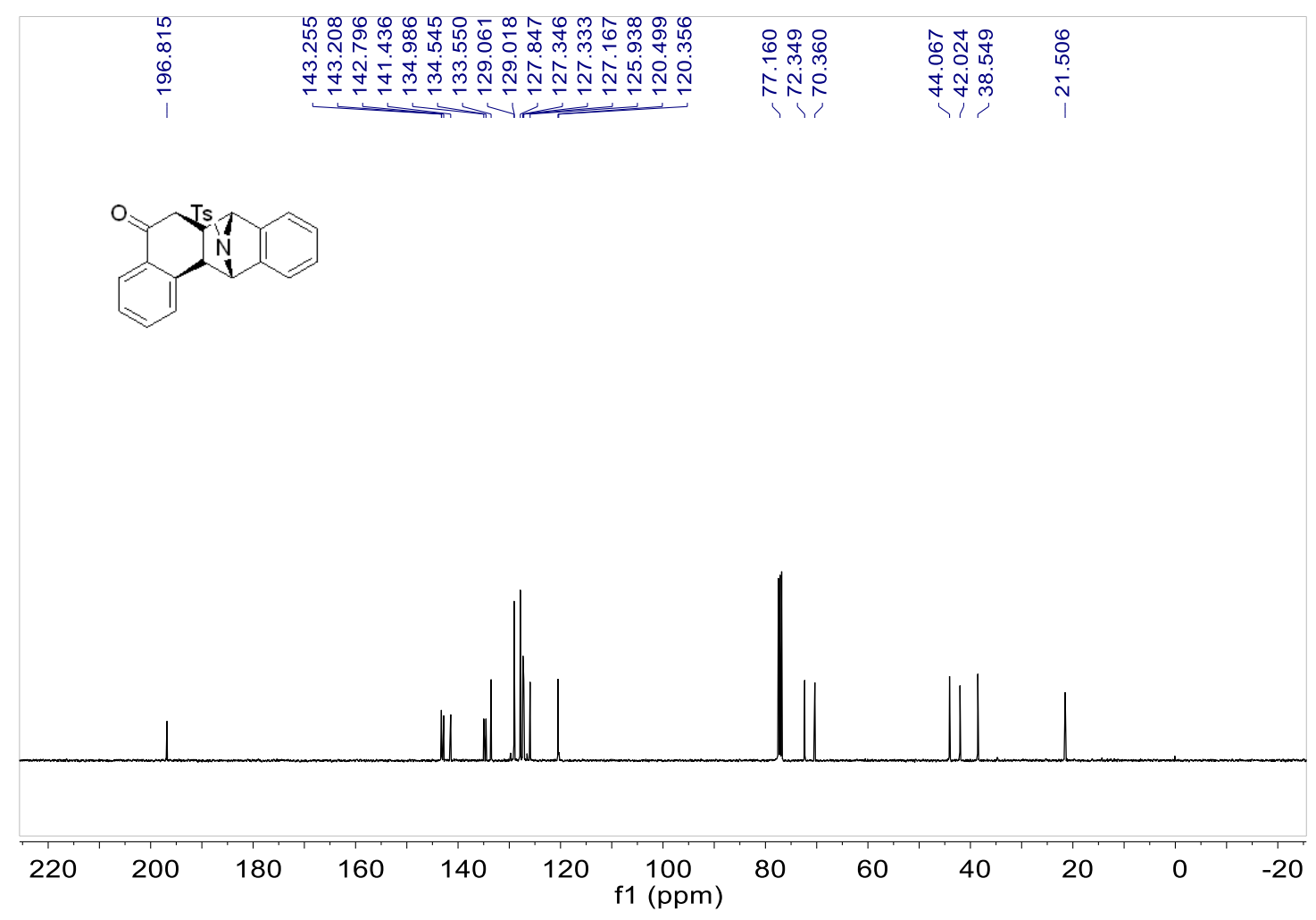

${ }^{13} \mathrm{C}$ NMR (100 MHz, $\left.\mathrm{CDCl}_{3}\right)$ spectrum of $5 \mathrm{aa}$ 


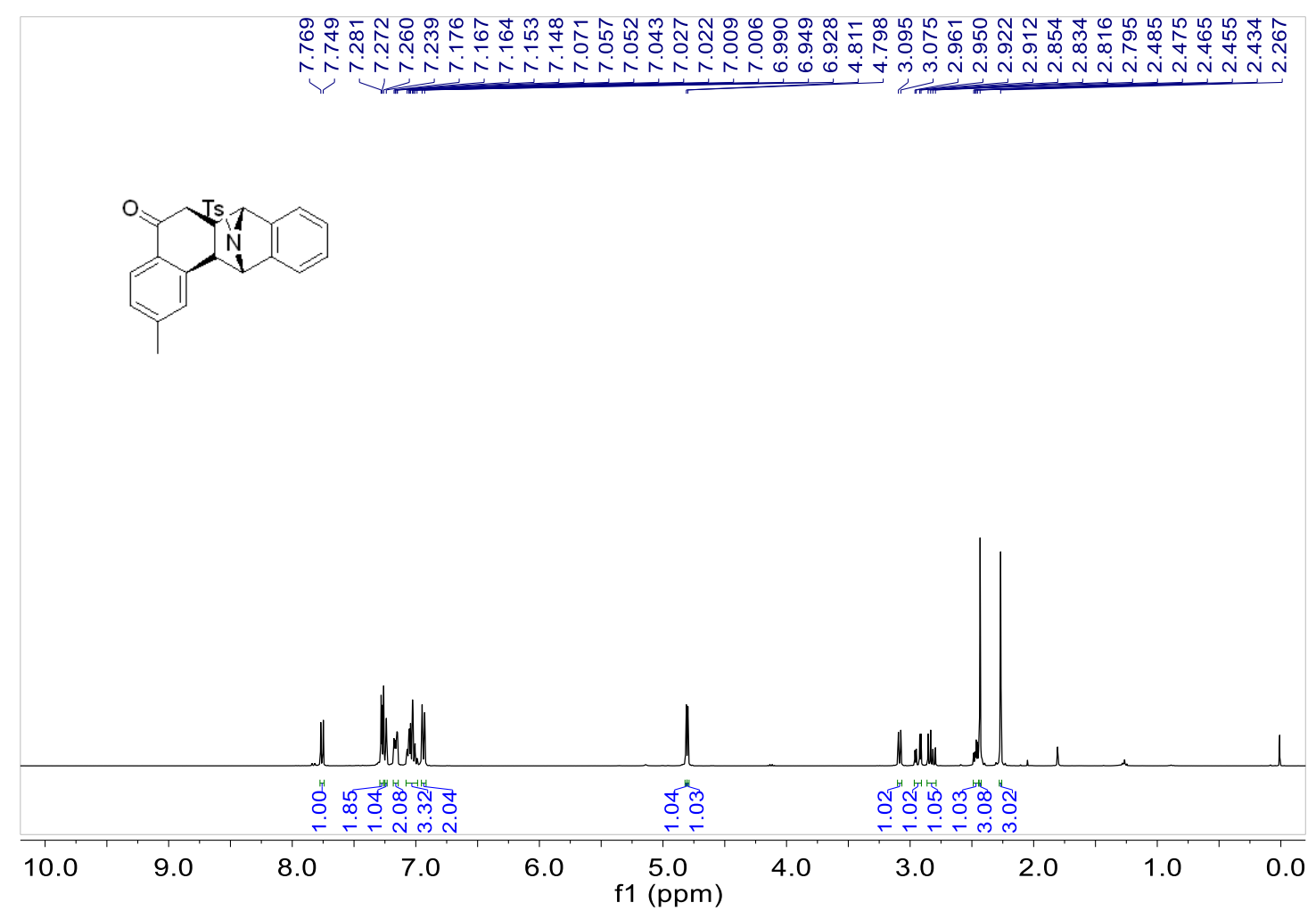

${ }^{1} \mathrm{H}$ NMR (400 MHz, $\mathrm{CDCl}_{3}$ ) spectrum of $5 \mathrm{ba}$

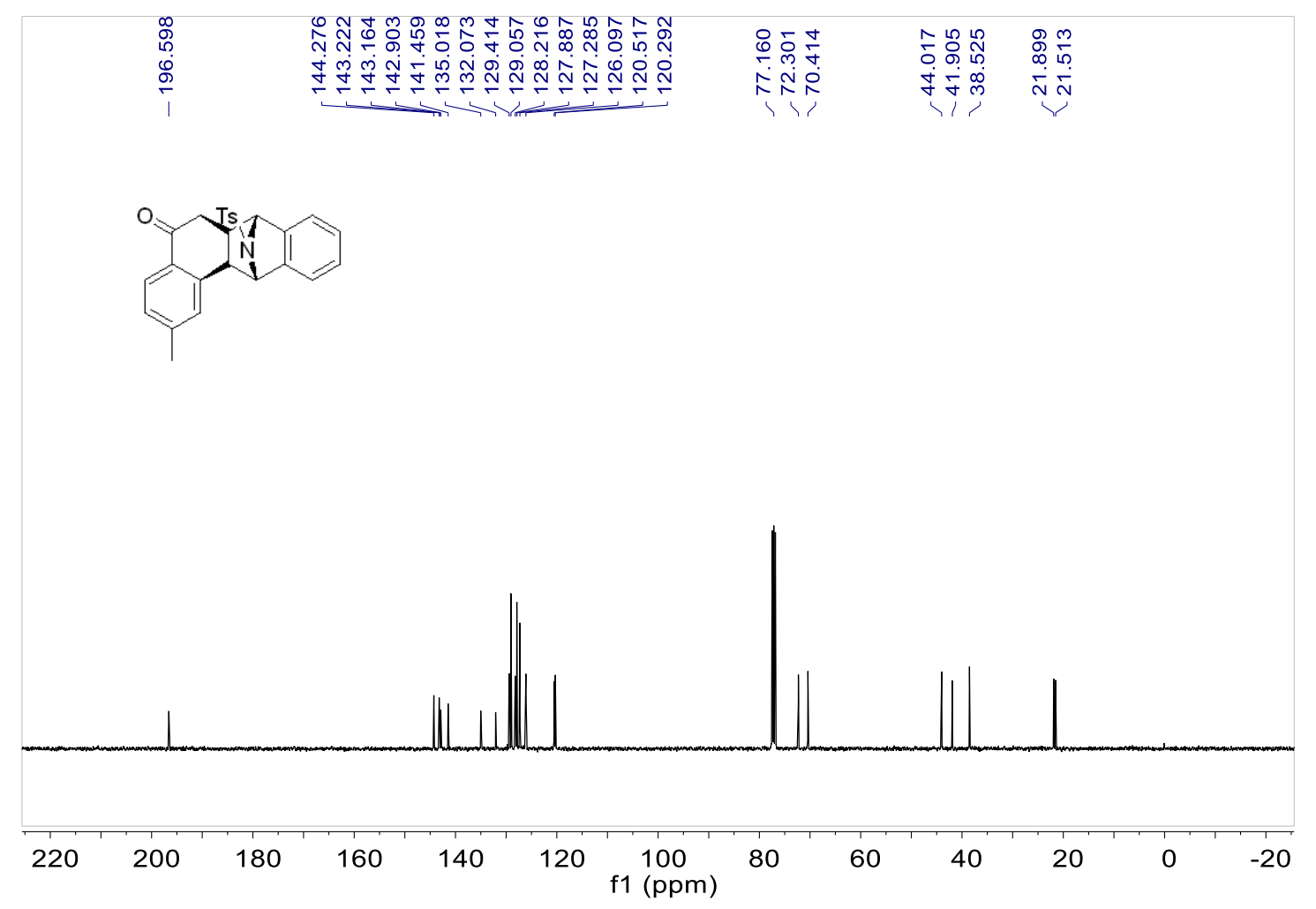

${ }^{13} \mathrm{C}$ NMR (100 MHz, $\mathrm{CDCl}_{3}$ ) spectrum of $5 \mathrm{ba}$ 

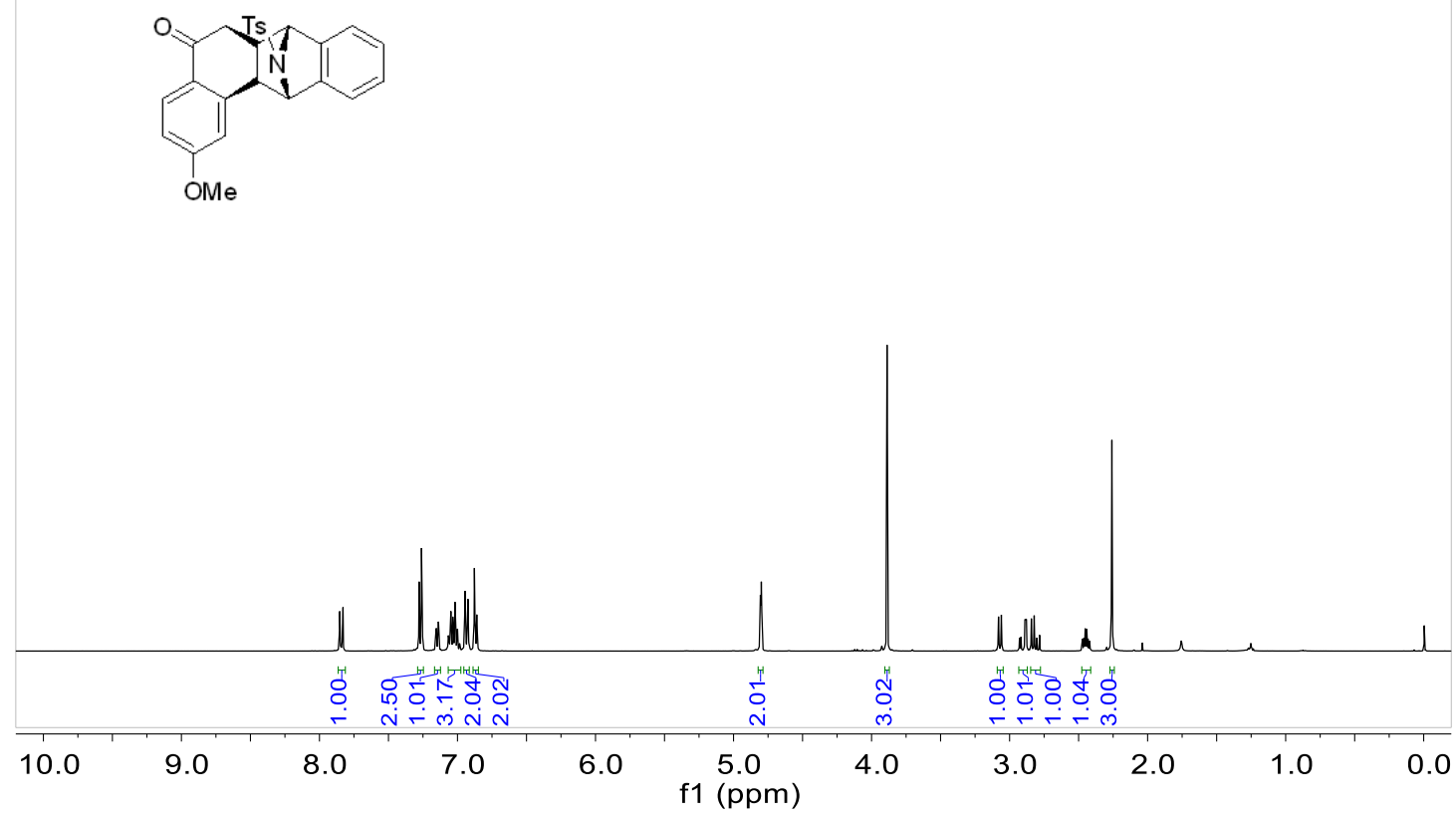

${ }^{1} \mathrm{H}$ NMR (400 MHz, $\mathrm{CDCl}_{3}$ ) spectrum of $5 \mathrm{ca}$

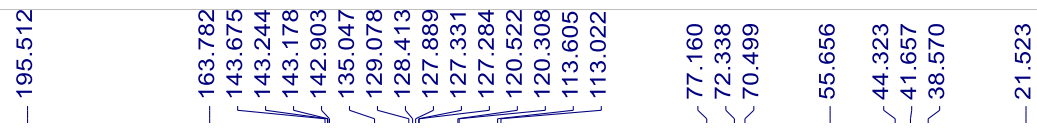

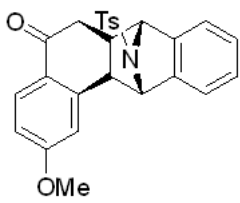
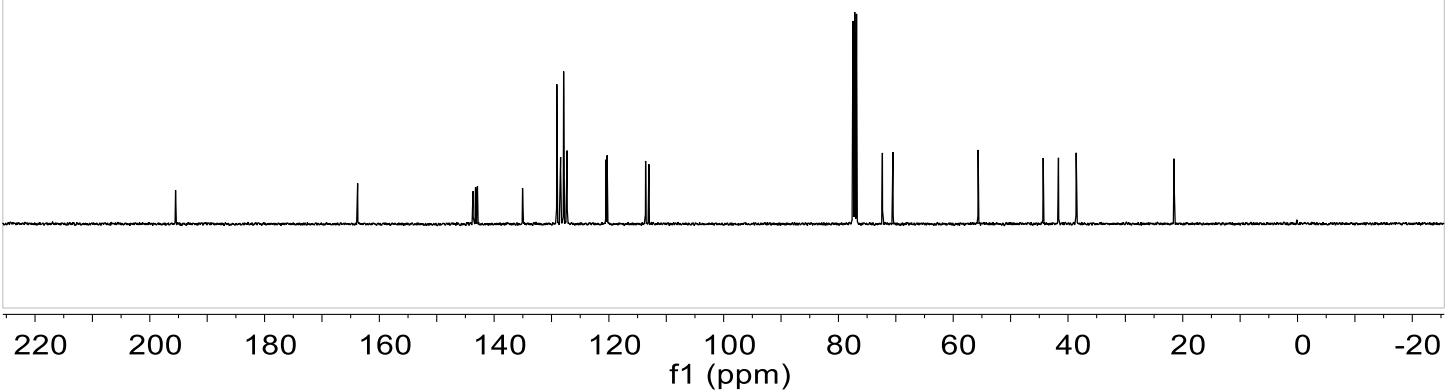

${ }^{13} \mathrm{C}$ NMR (100 MHz, $\left.\mathrm{CDCl}_{3}\right)$ spectrum of $5 \mathrm{ca}$ 

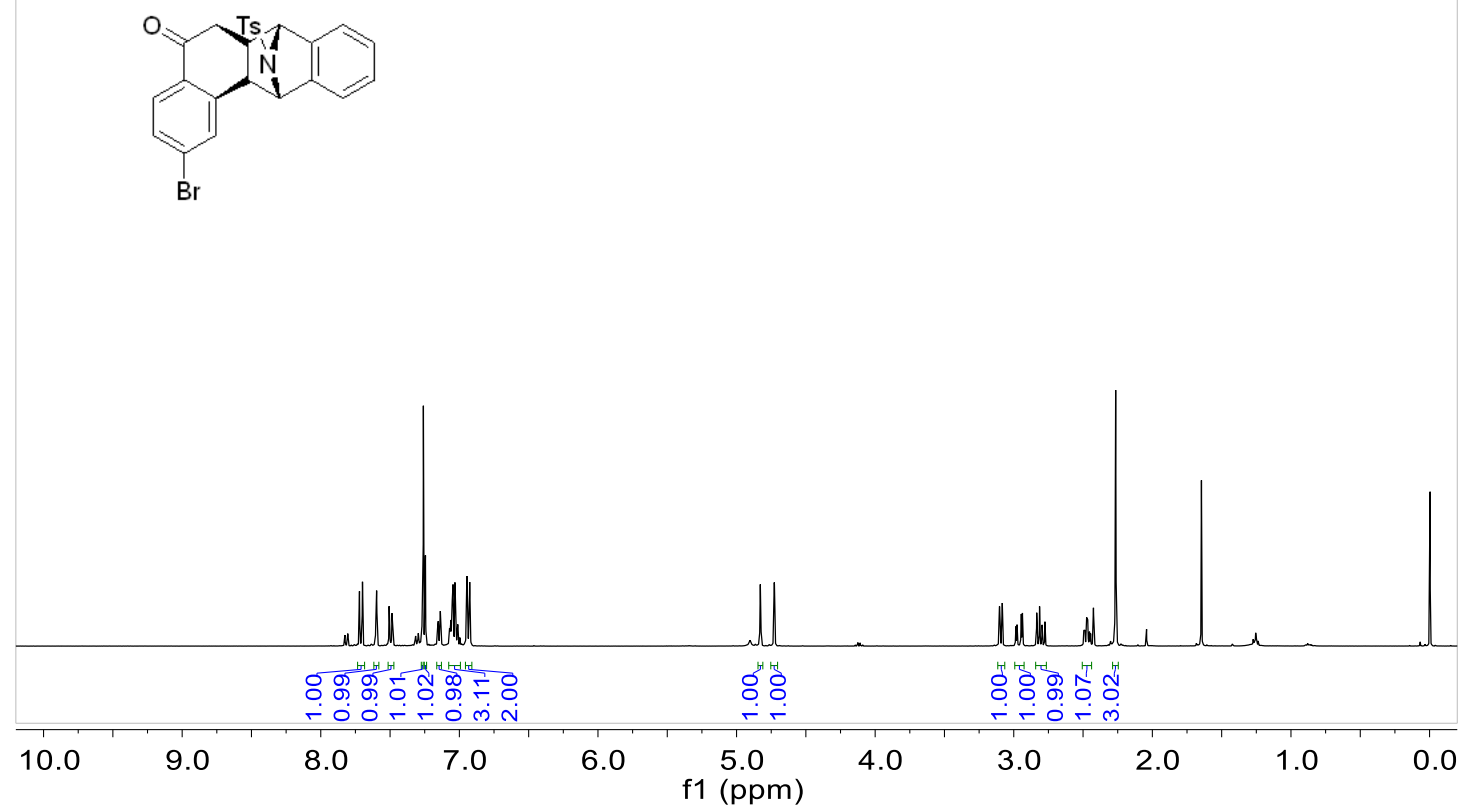

${ }^{1} \mathrm{H}$ NMR (400 MHz, $\mathrm{CDCl}_{3}$ ) spectrum of $5 \mathrm{da}$

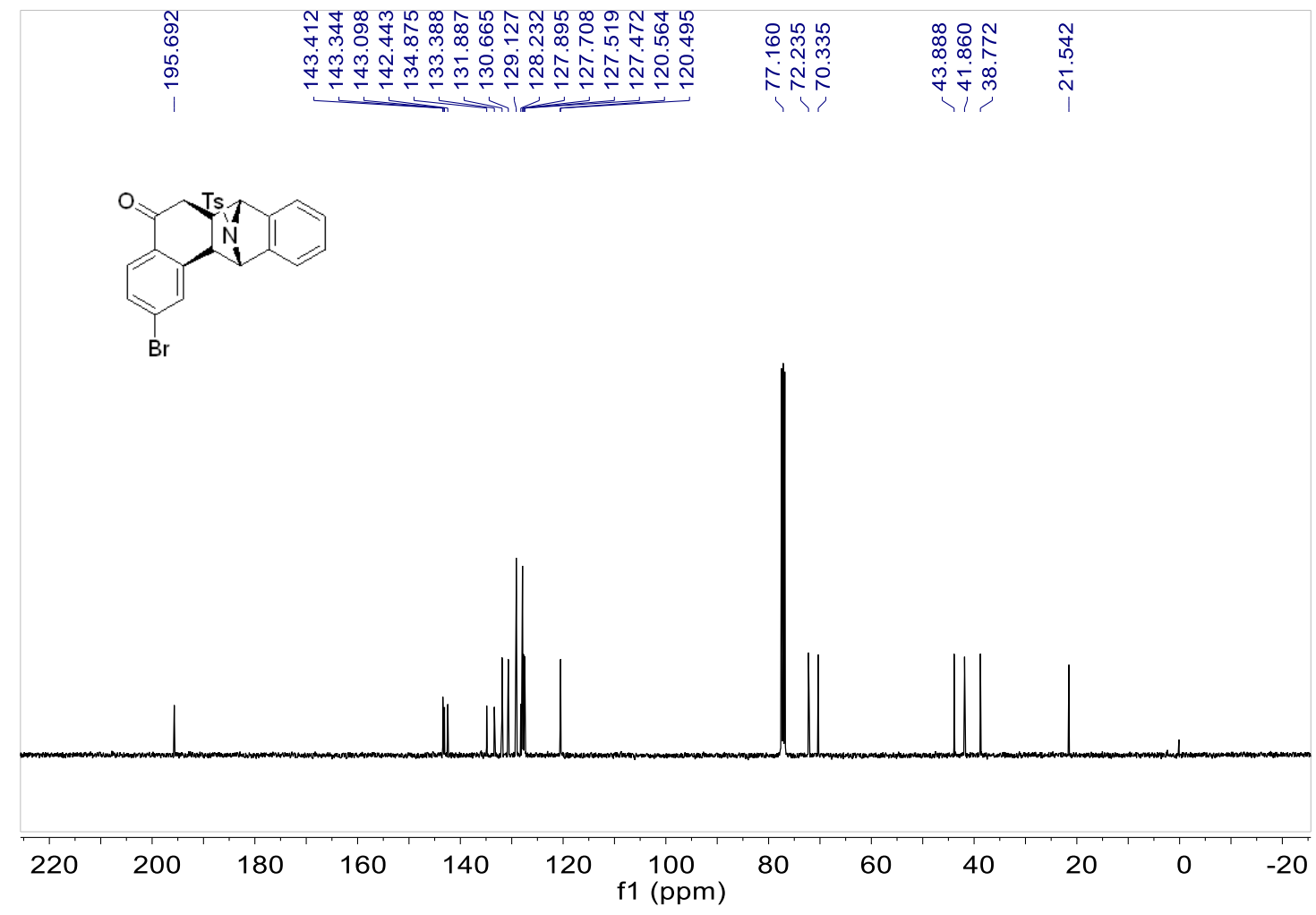

${ }^{13} \mathrm{C}$ NMR (100 MHz, $\left.\mathrm{CDCl}_{3}\right)$ spectrum of $5 \mathrm{da}$ 


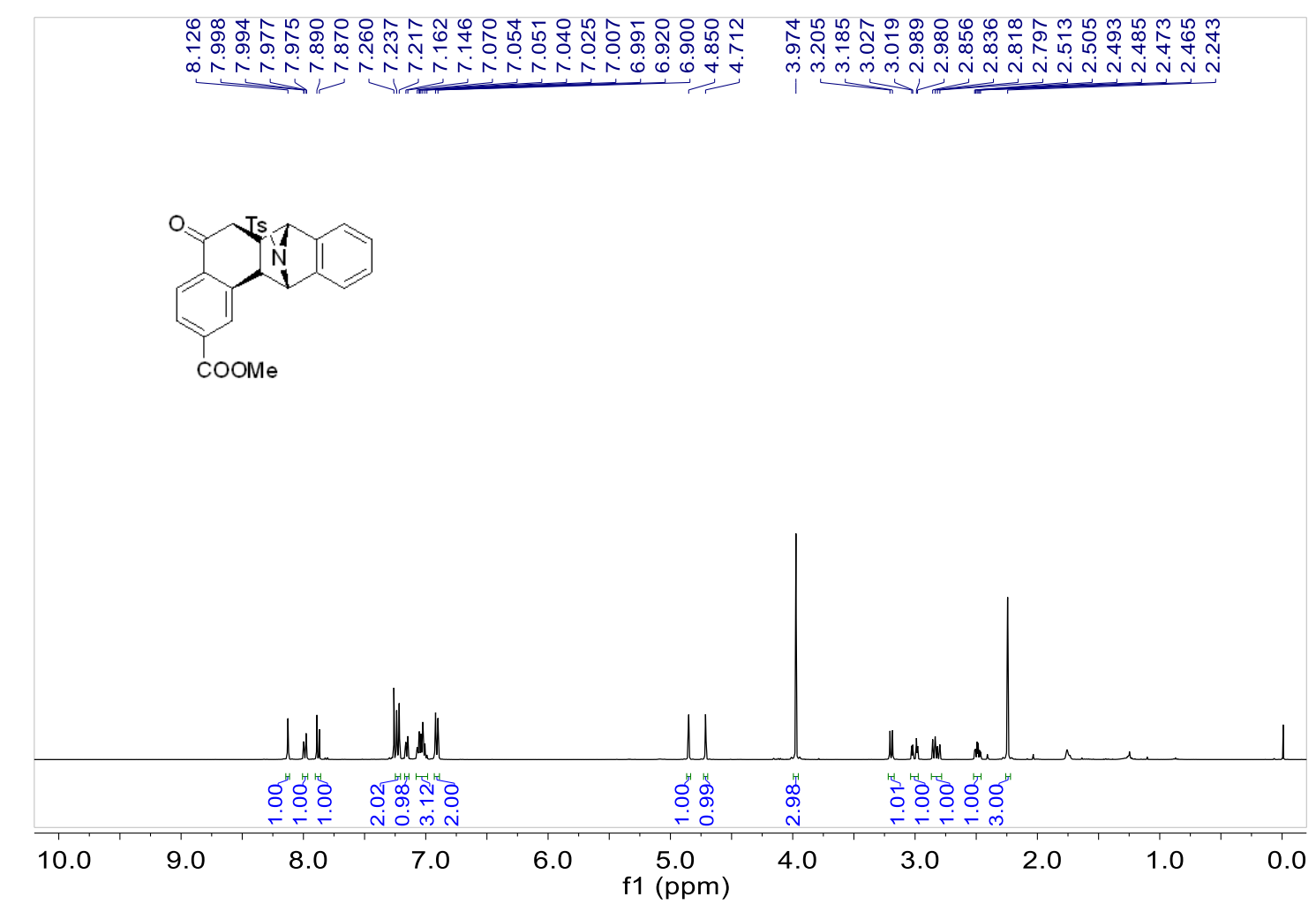

${ }^{1} \mathrm{H}$ NMR (400 MHz, $\mathrm{CDCl}_{3}$ ) spectrum of $5 \mathrm{ea}$

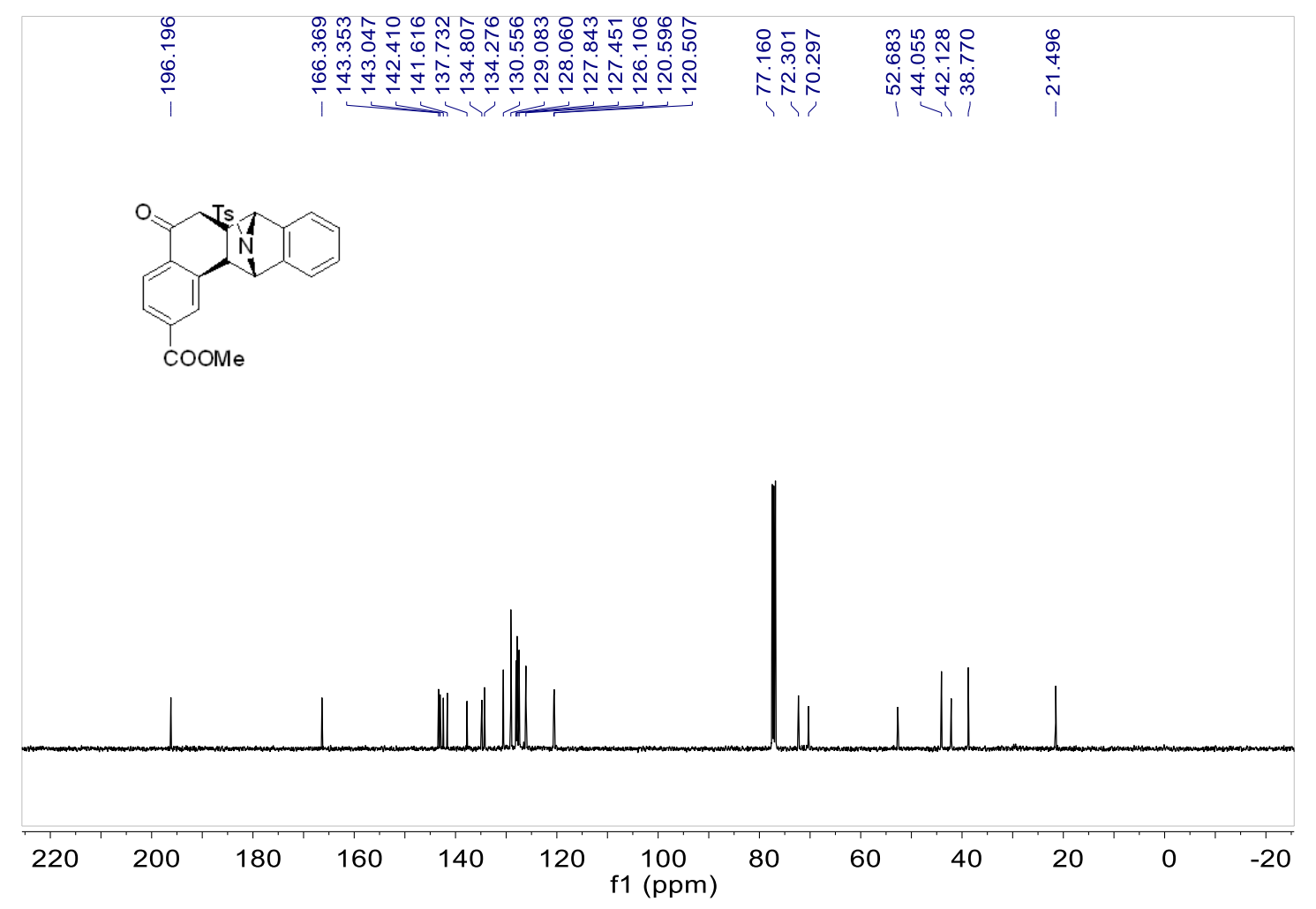

${ }^{13} \mathrm{C}$ NMR (100 MHz, $\mathrm{CDCl}_{3}$ ) spectrum of $5 \mathrm{ea}$ 
<smiles>COc1cccc2c1C(=O)C1[C+]3c4ccccc4C1c1ccccc1C23</smiles>

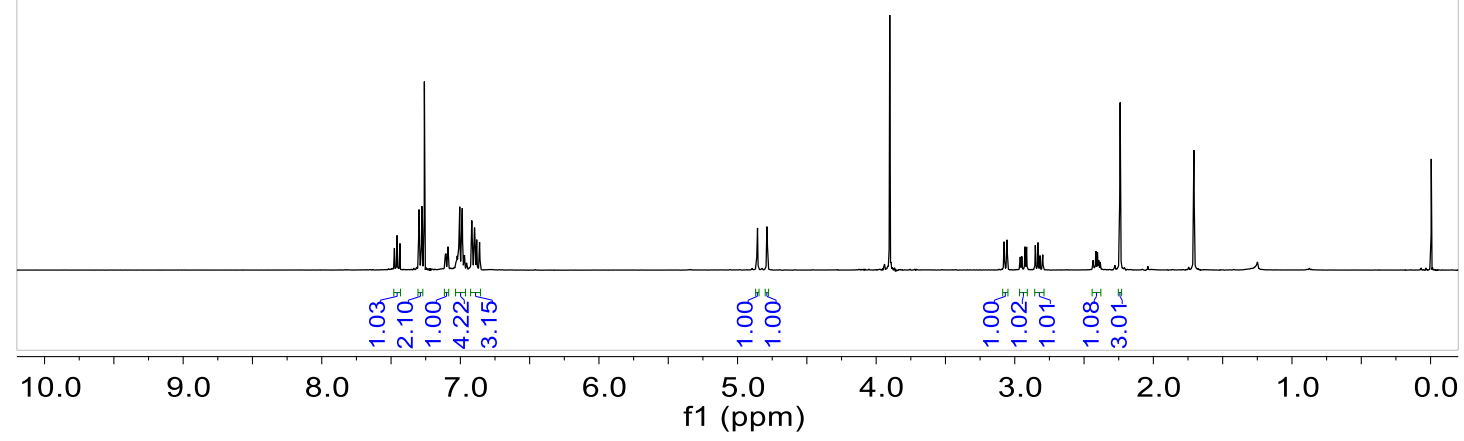

${ }^{1} \mathrm{H}$ NMR (400 MHz, $\left.\mathrm{CDCl}_{3}\right)$ spectrum of $5 f a$

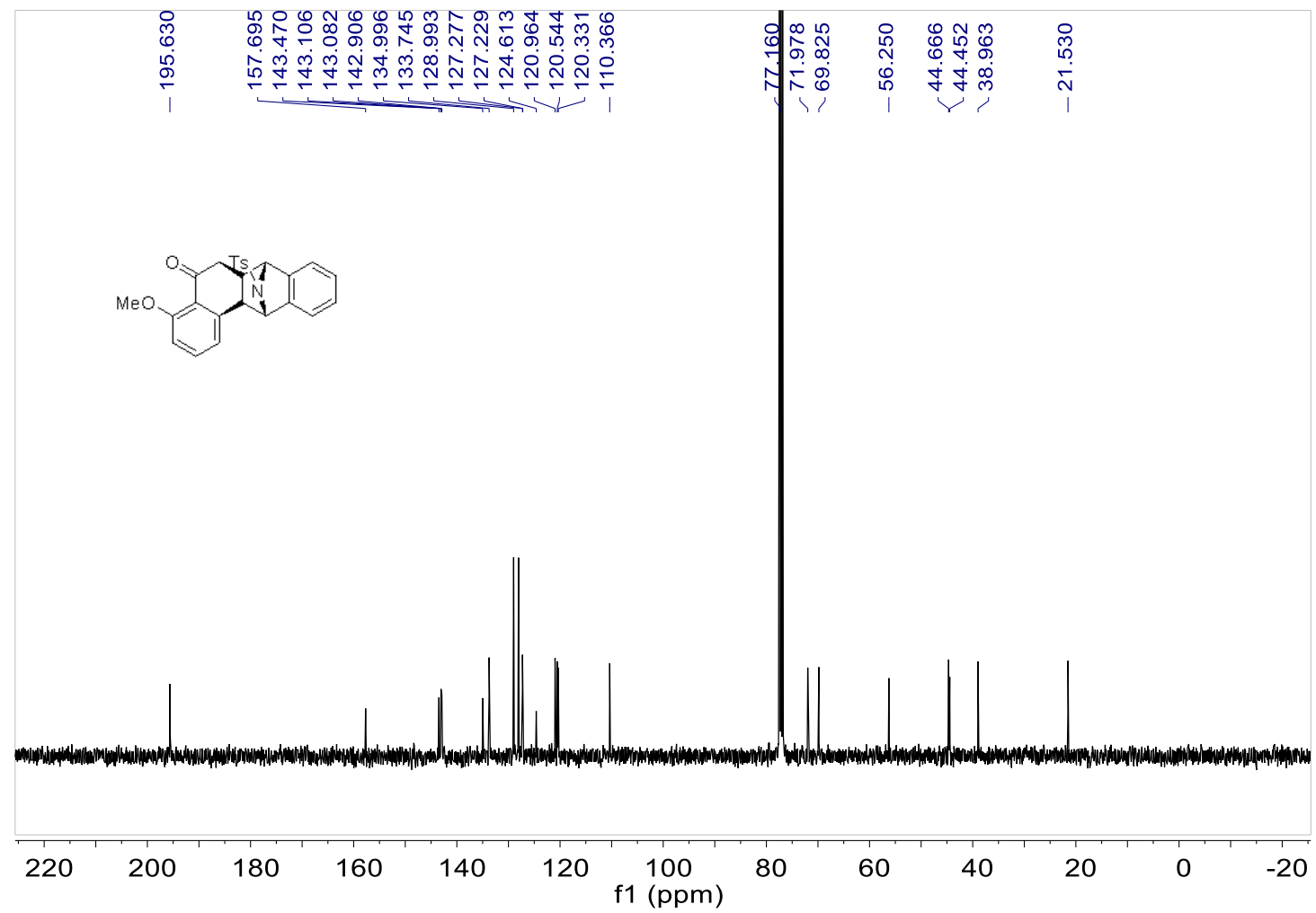

${ }^{13} \mathrm{C}$ NMR (100 MHz, $\left.\mathrm{CDCl}_{3}\right)$ spectrum of $5 f a$ 

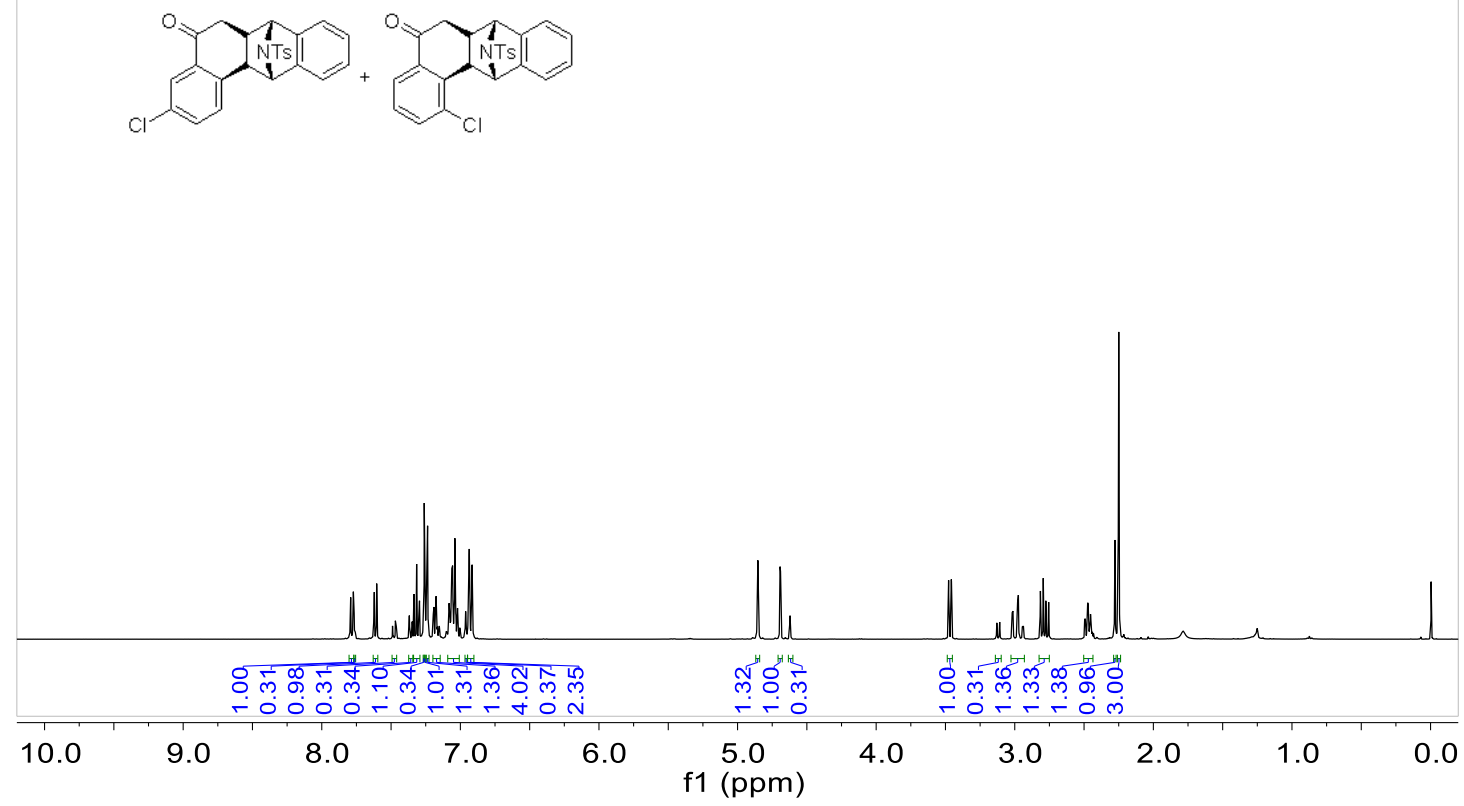

${ }^{1} \mathrm{H}$ NMR (400 MHz, $\mathrm{CDCl}_{3}$ ) spectrum of $5 \mathrm{ga}$

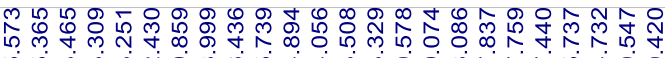

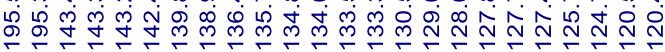

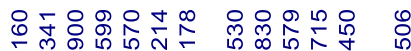

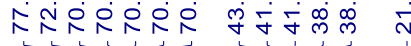

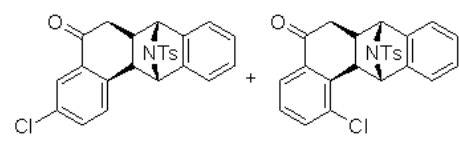

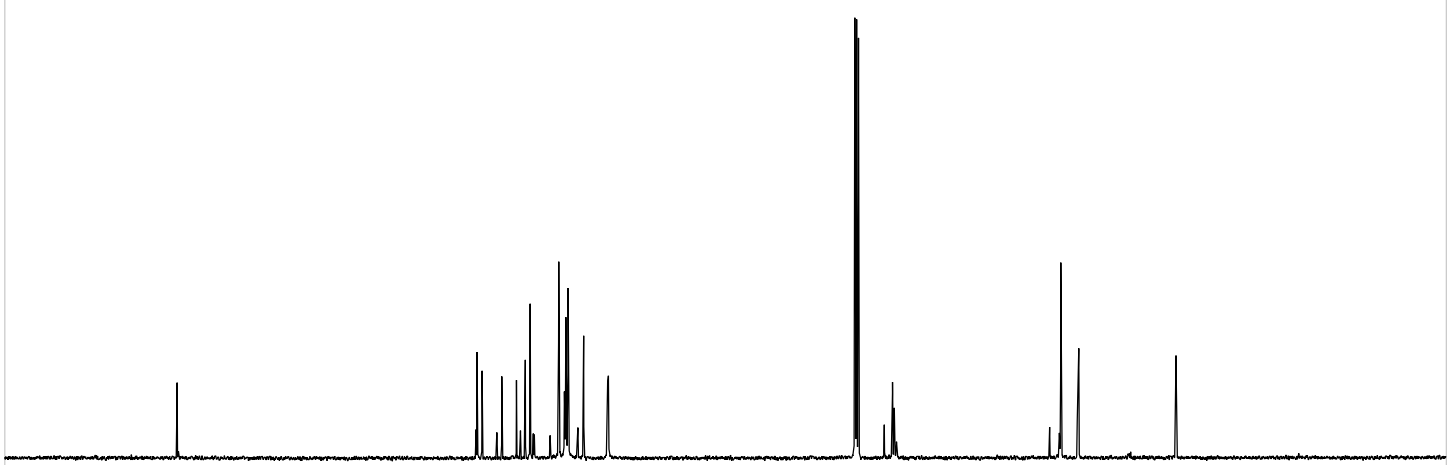

220

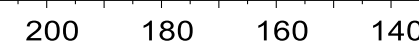

120 f1 $\left(\begin{array}{l}100 \\ (p p m)\end{array}\right.$

${ }^{13} \mathrm{C}$ NMR (100 MHz, $\mathrm{CDCl}_{3}$ ) spectrum of $5 \mathrm{ga}$ 


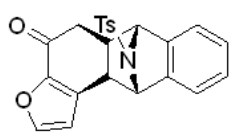

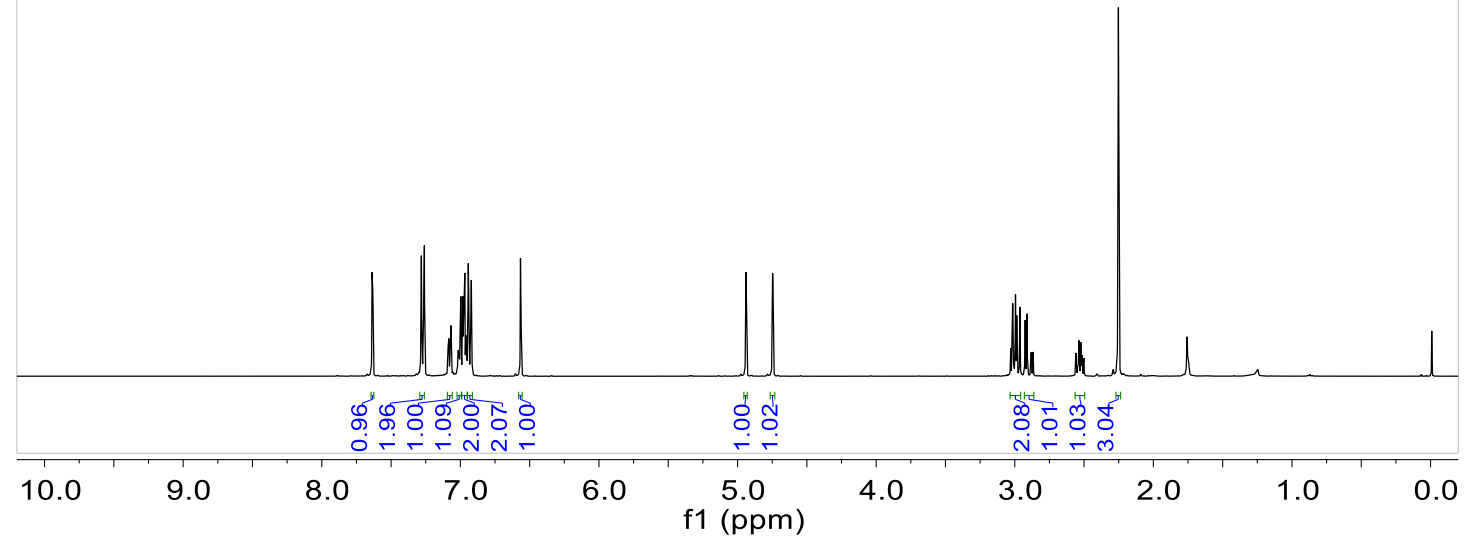

${ }^{1} \mathrm{H}$ NMR (400 MHz, $\mathrm{CDCl}_{3}$ ) spectrum of 5 ha

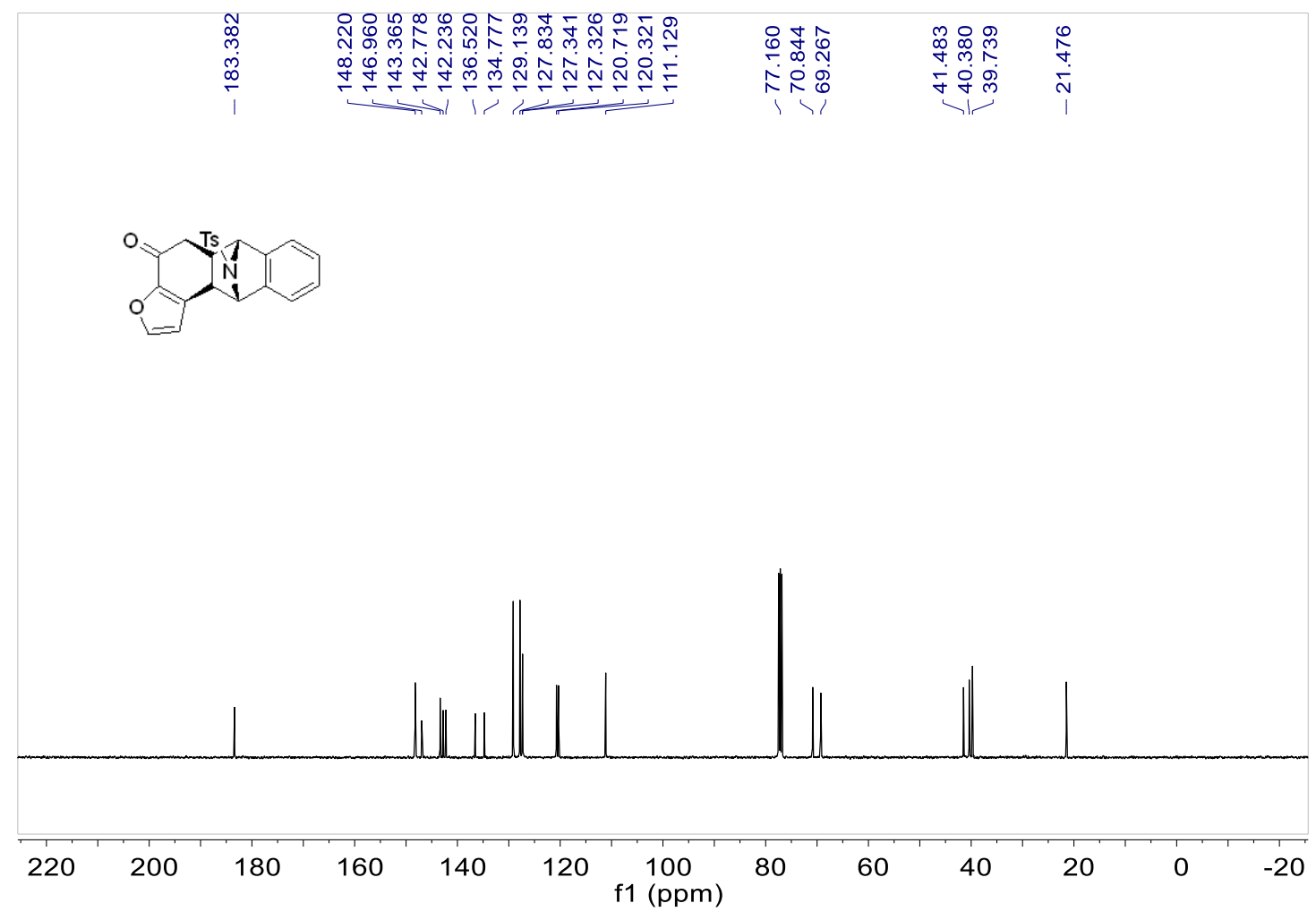

${ }^{13} \mathrm{C}$ NMR (100 MHz, $\left.\mathrm{CDCl}_{3}\right)$ spectrum of 5 ha 
<smiles>O=C1C[n+]2c3cc(c4ccccc42)C1=C3</smiles>

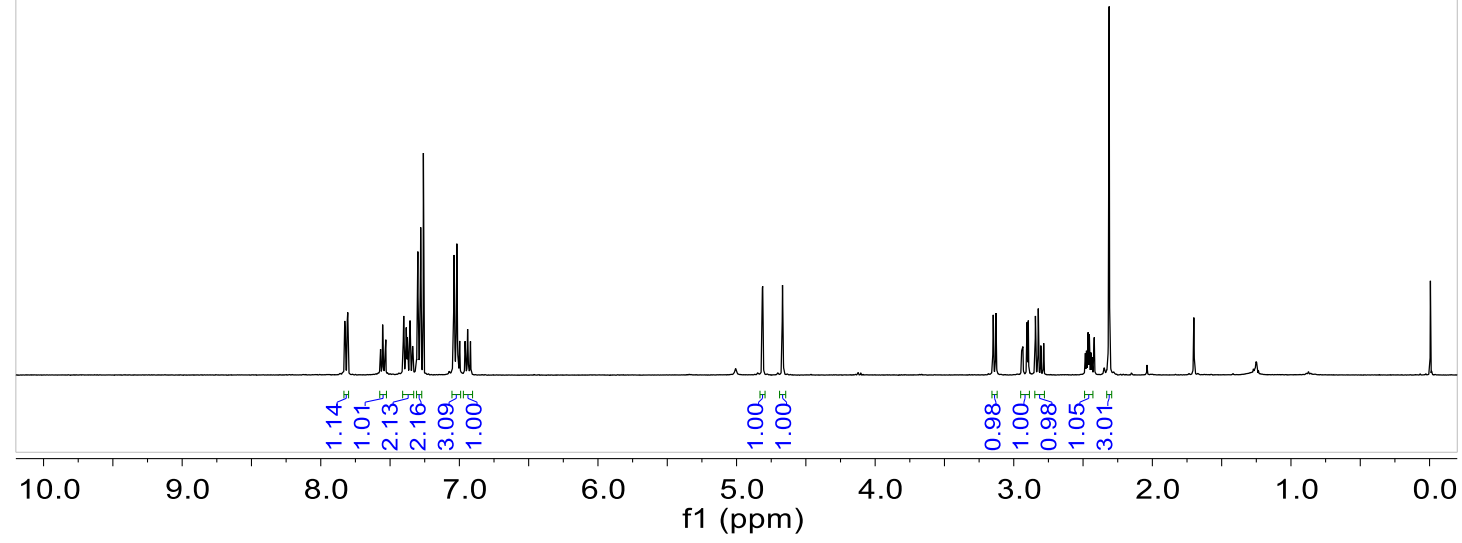

${ }^{1} \mathrm{H}$ NMR (400 MHz, $\mathrm{CDCl}_{3}$ ) spectrum of $5 \mathrm{ab}$

\begin{tabular}{|c|c|c|}
\hline 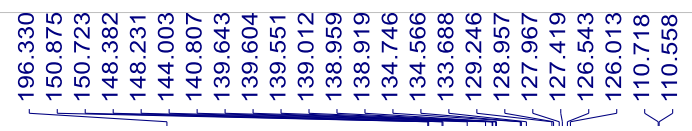 & 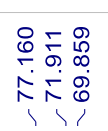 & 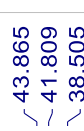 \\
\hline
\end{tabular}<smiles>O=C1C[N+]23CCC2c2cc(F)c(F)cc2C13</smiles>

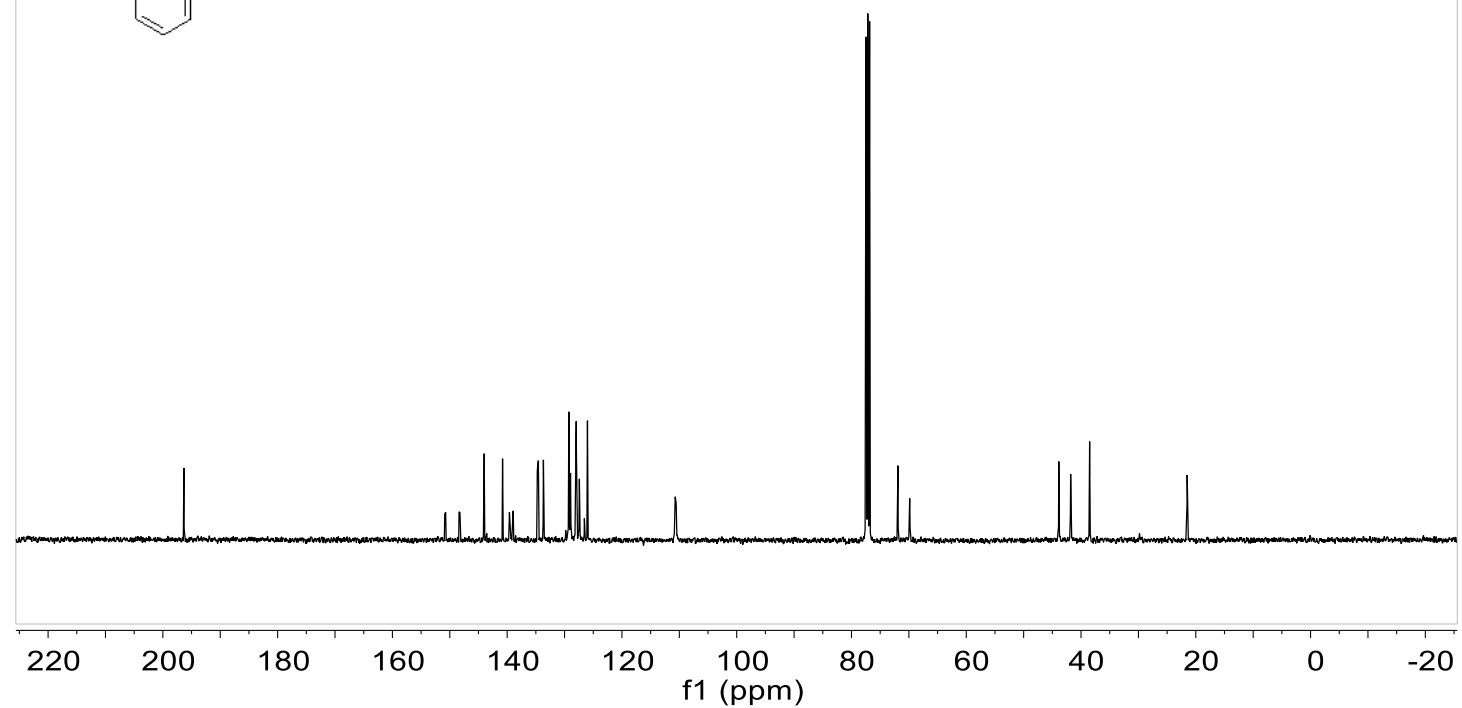

${ }^{13} \mathrm{C}$ NMR (100 MHz, $\left.\mathrm{CDCl}_{3}\right)$ spectrum of $5 \mathrm{ab}$ 


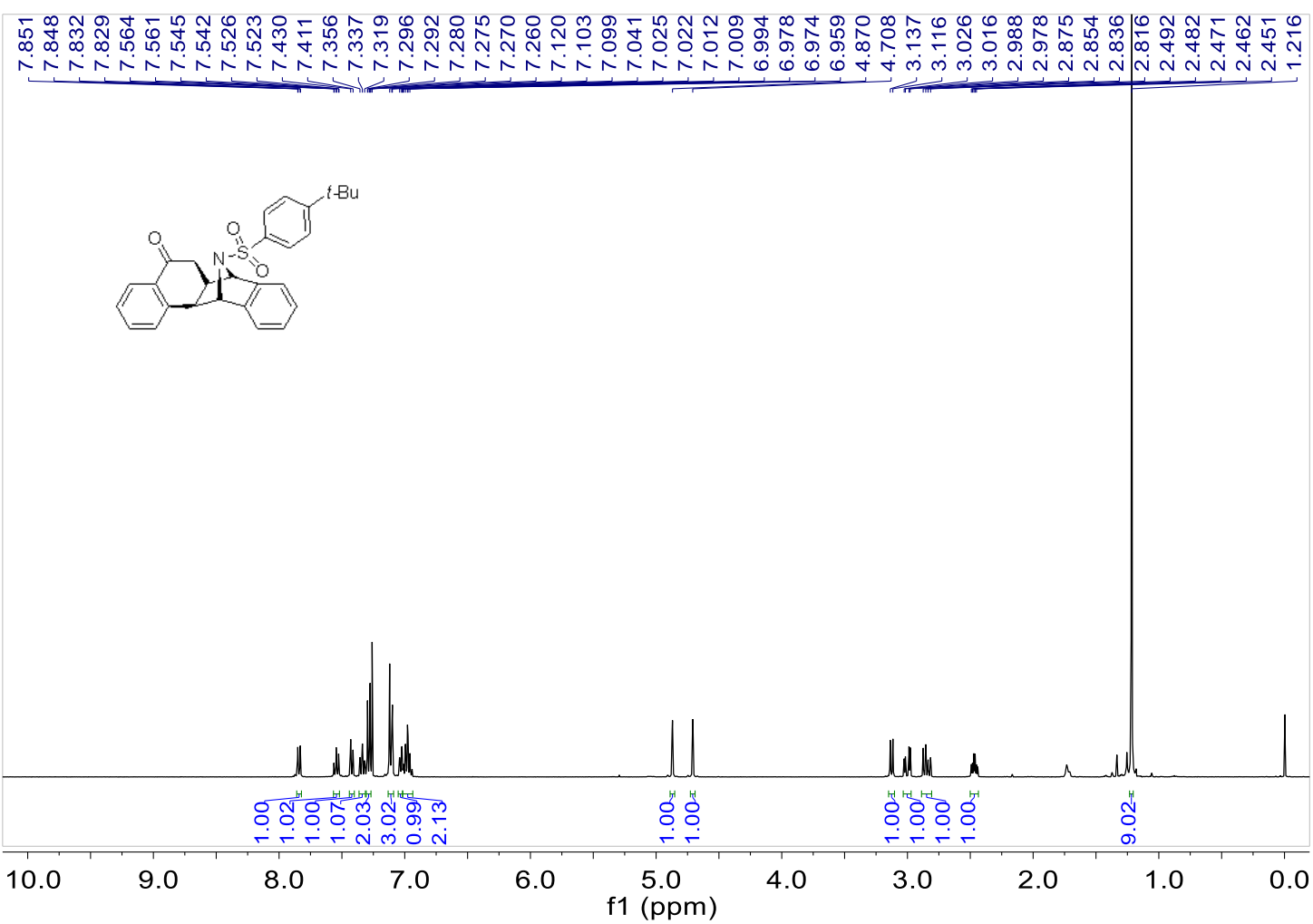

${ }^{1} \mathrm{H}$ NMR (400 MHz, $\mathrm{CDCl}_{3}$ ) spectrum of $5 \mathrm{ac}$

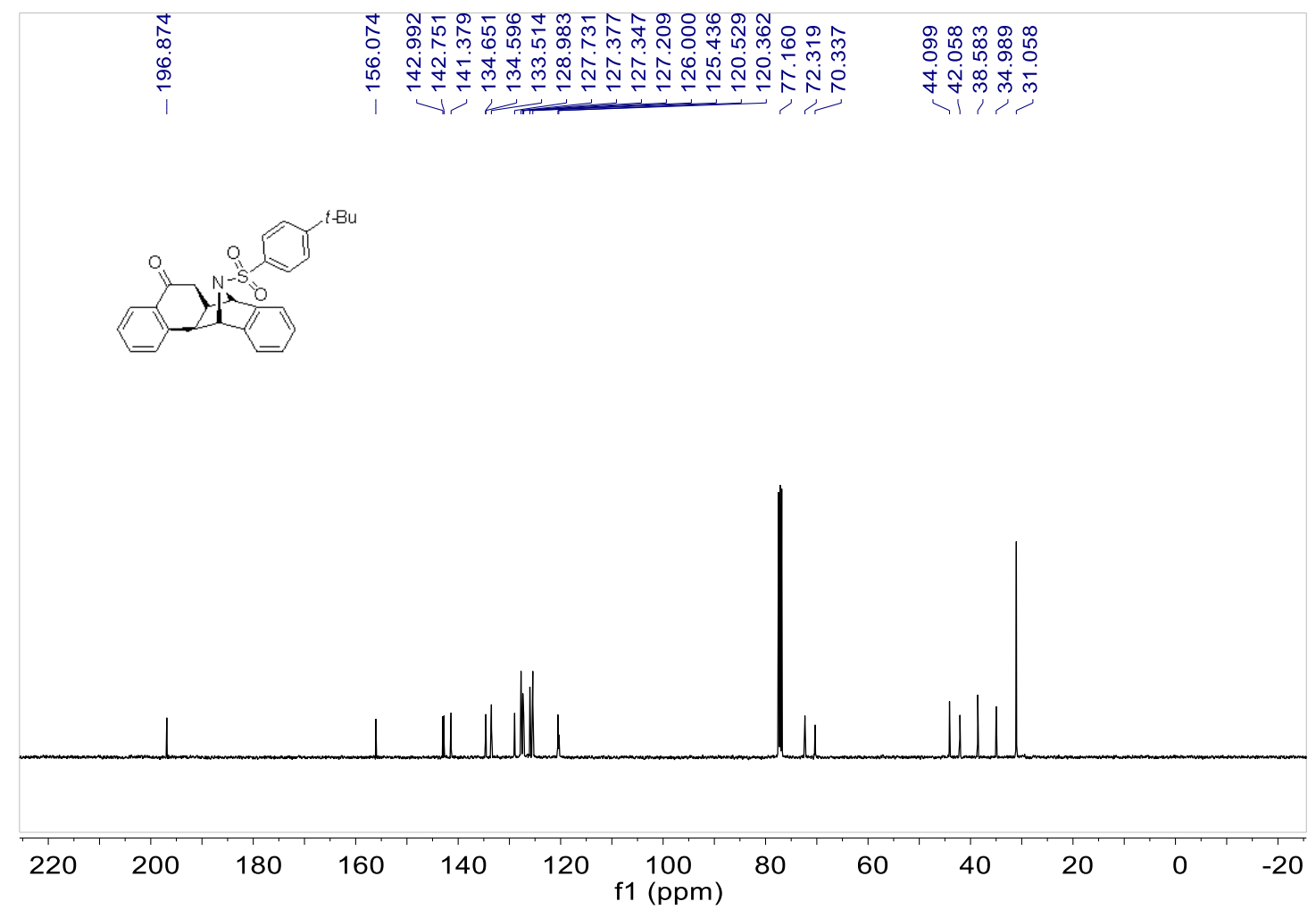

${ }^{13} \mathrm{C}$ NMR (100 MHz, $\mathrm{CDCl}_{3}$ ) spectrum of $5 \mathrm{ac}$ 


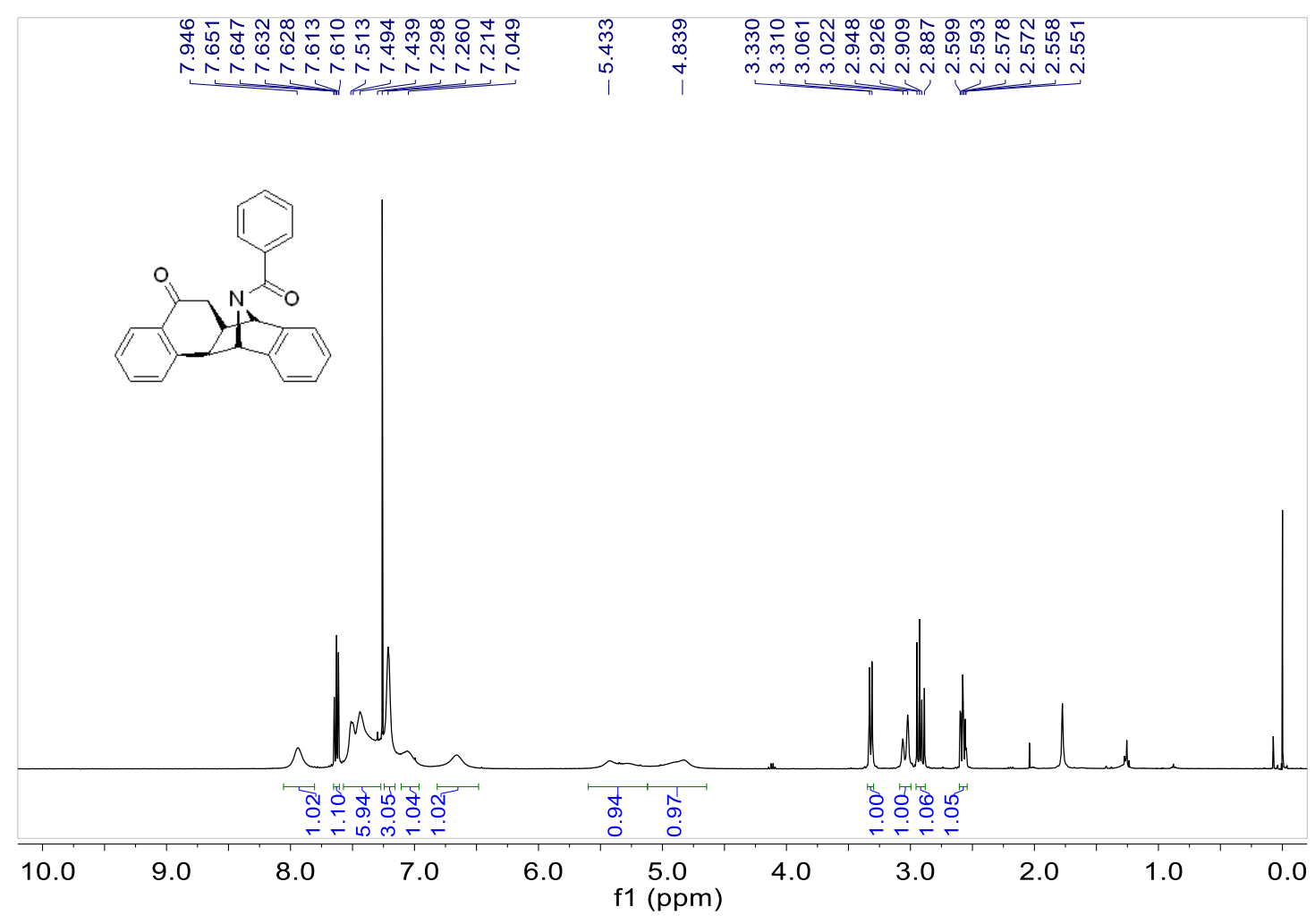

${ }^{1} \mathrm{H}$ NMR (400 MHz, $\mathrm{CDCl}_{3}$ ) spectrum of $5 \mathrm{ad}$
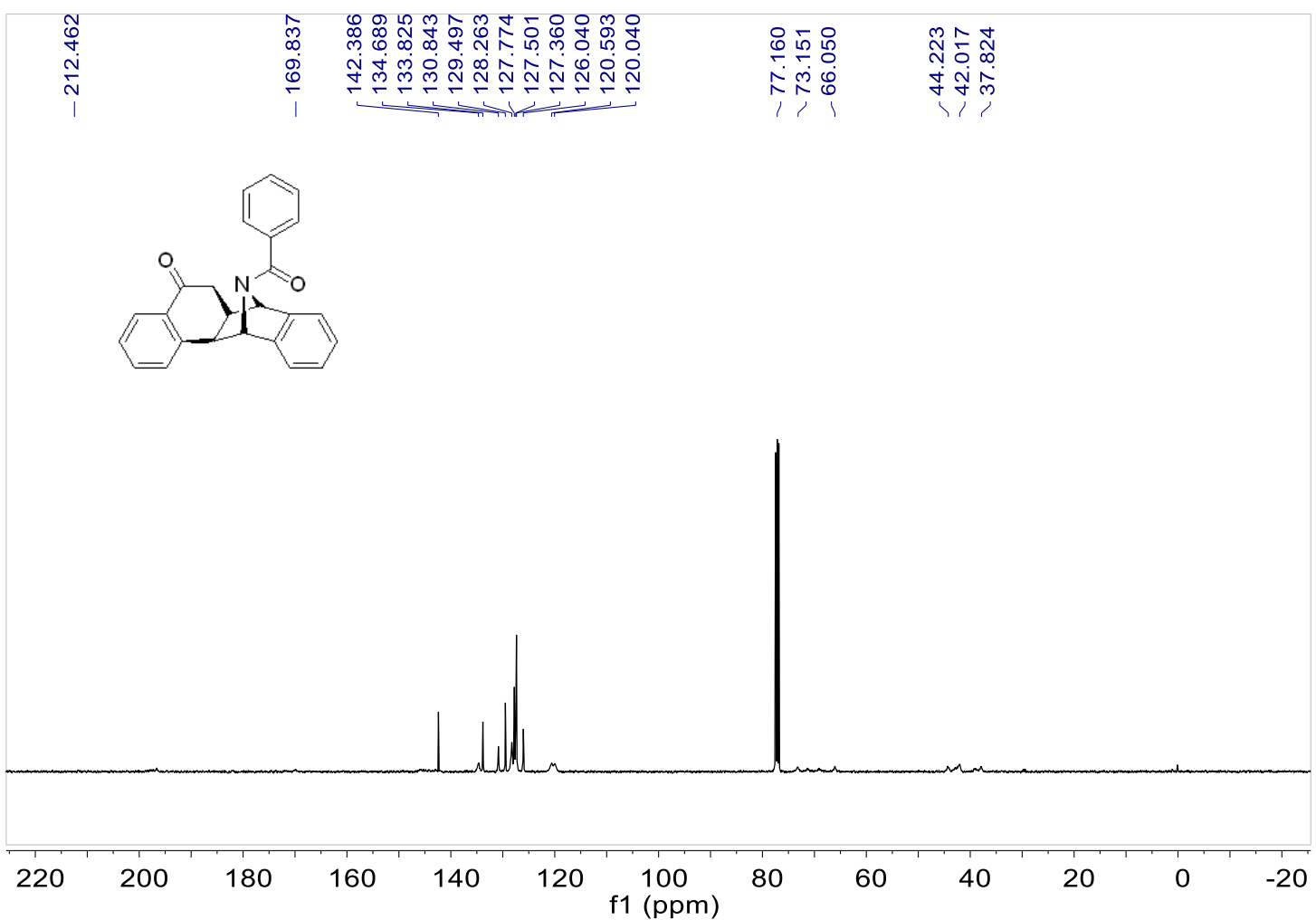

${ }^{13} \mathrm{C}$ NMR (100 MHz, $\left.\mathrm{CDCl}_{3}\right)$ spectrum of $5 \mathrm{ad}$ 


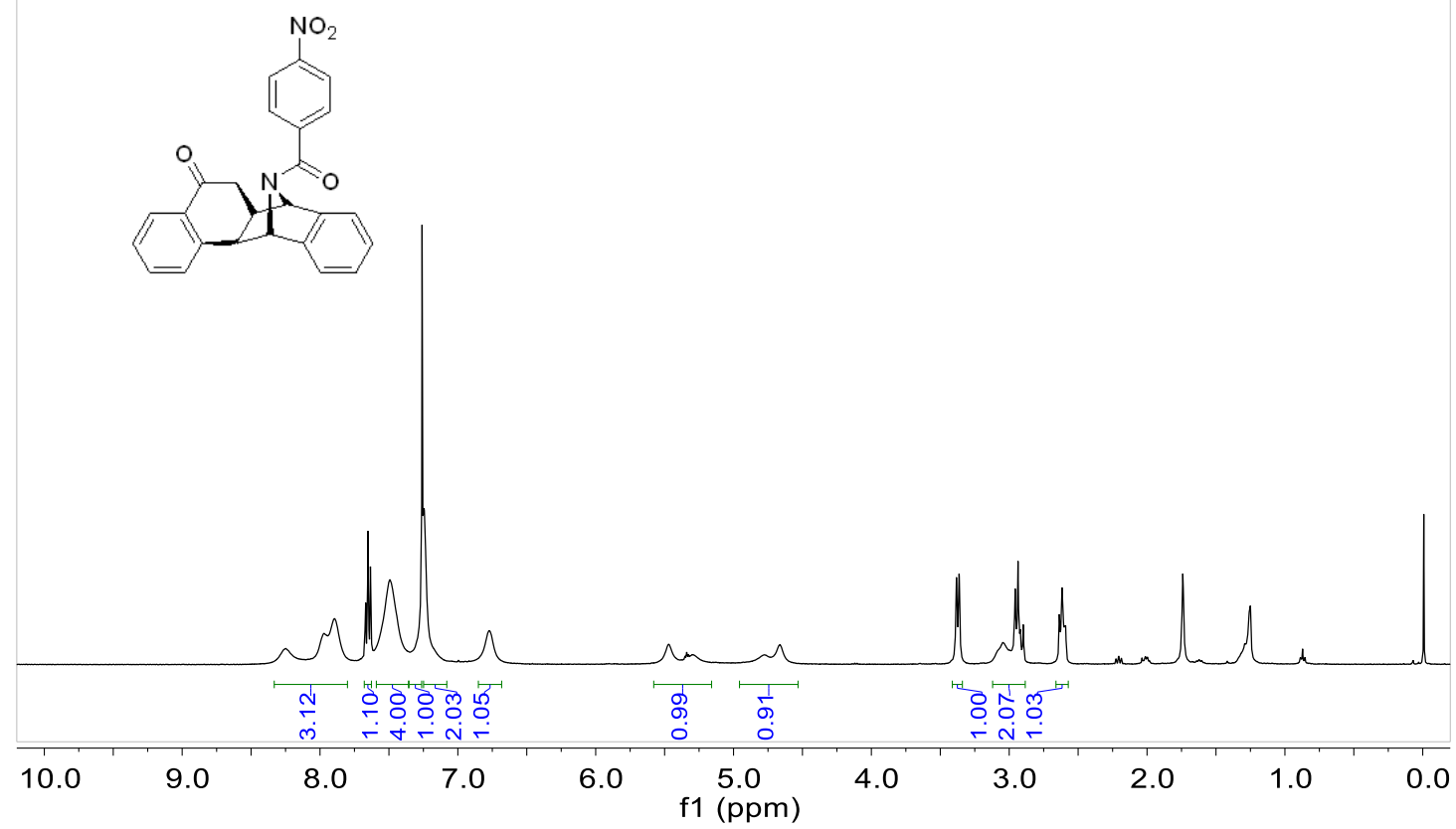

${ }^{1} \mathrm{H}$ NMR (400 MHz, $\left.\mathrm{CDCl}_{3}\right)$ spectrum of $5 \mathrm{ae}$

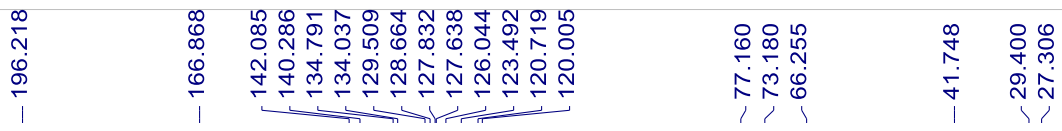
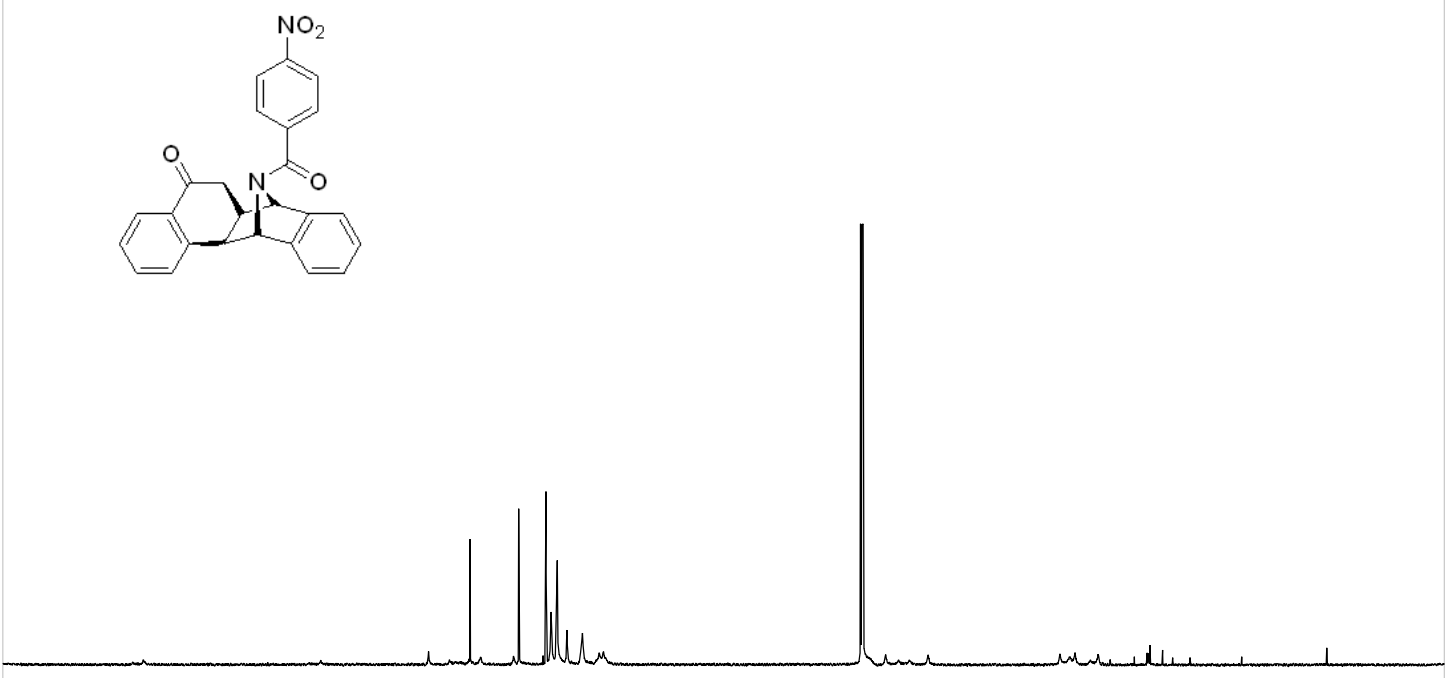

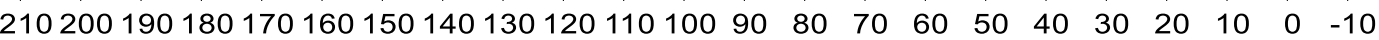
f1 (ppm)

$\left.{ }^{13} \mathrm{C} \mathrm{NMR} \mathrm{(150} \mathrm{MHz,} \mathrm{CDCl}_{3}\right)$ spectrum of $5 \mathrm{ae}$ 


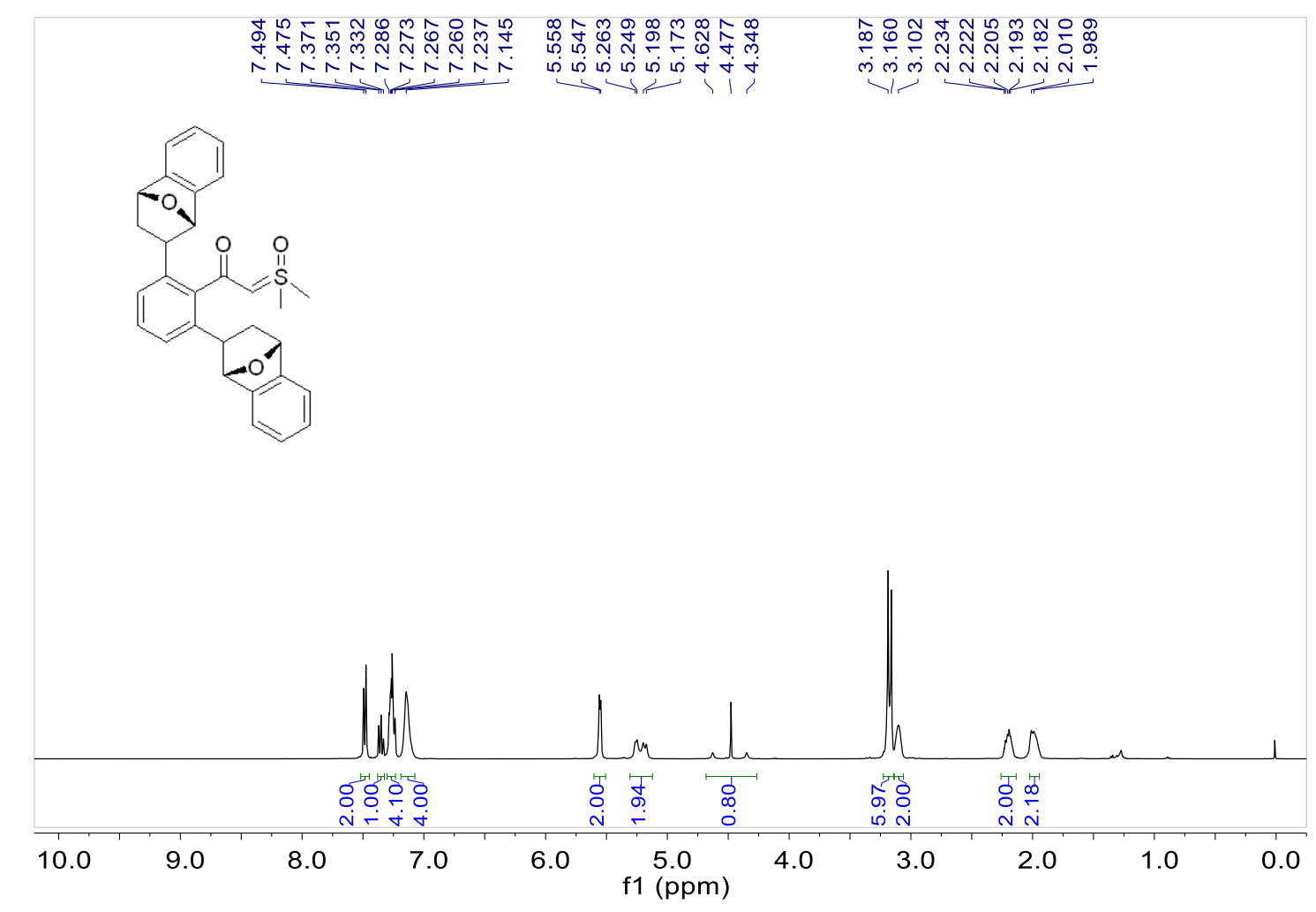

${ }^{1} \mathrm{H}$ NMR (400 MHz, $\mathrm{CDCl}_{3}$ ) spectrum of $6 \mathrm{aa}$

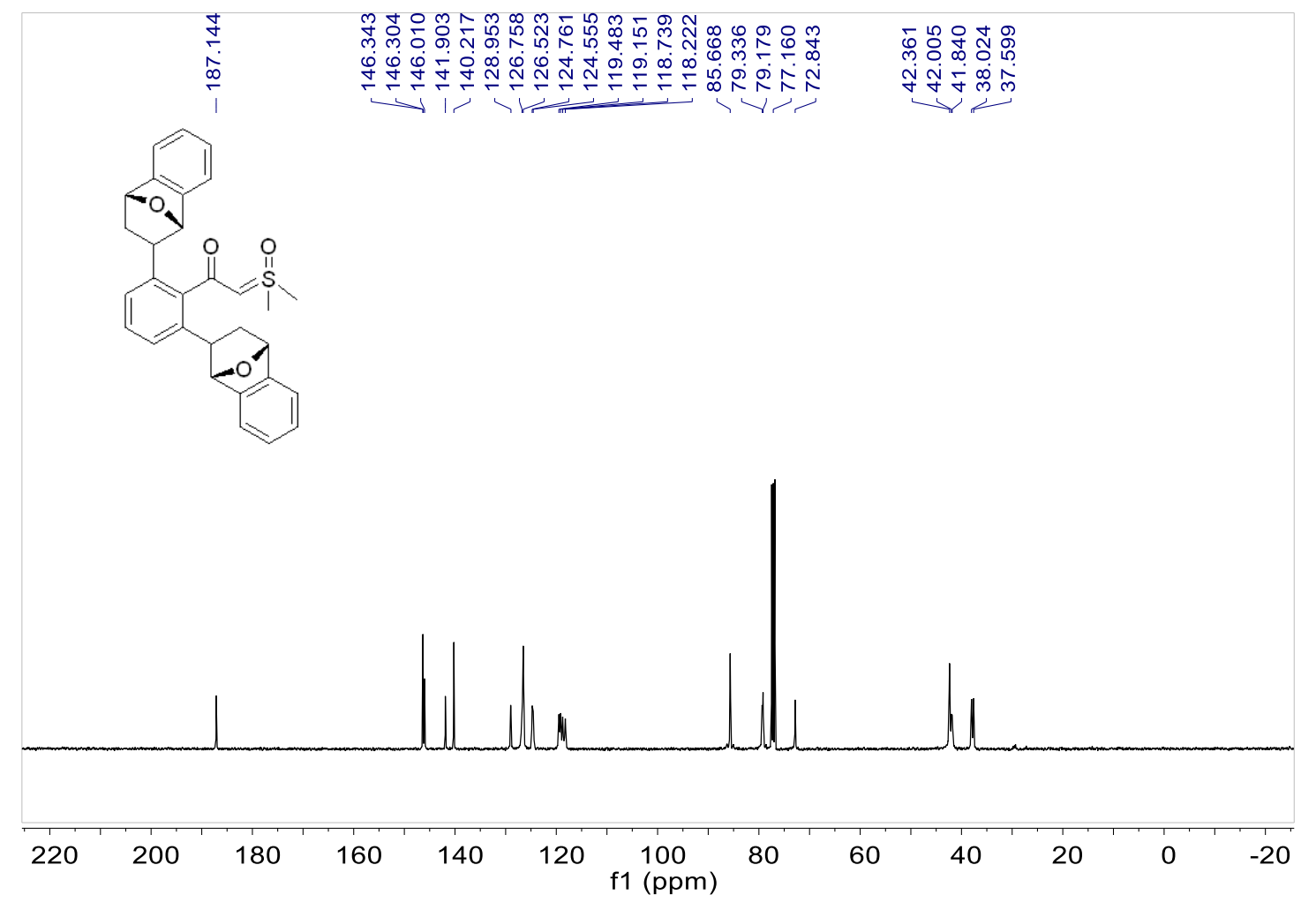

${ }^{13} \mathrm{C}$ NMR (100 MHz, $\mathrm{CDCl}_{3}$ ) spectrum of $6 \mathrm{aa}$ 


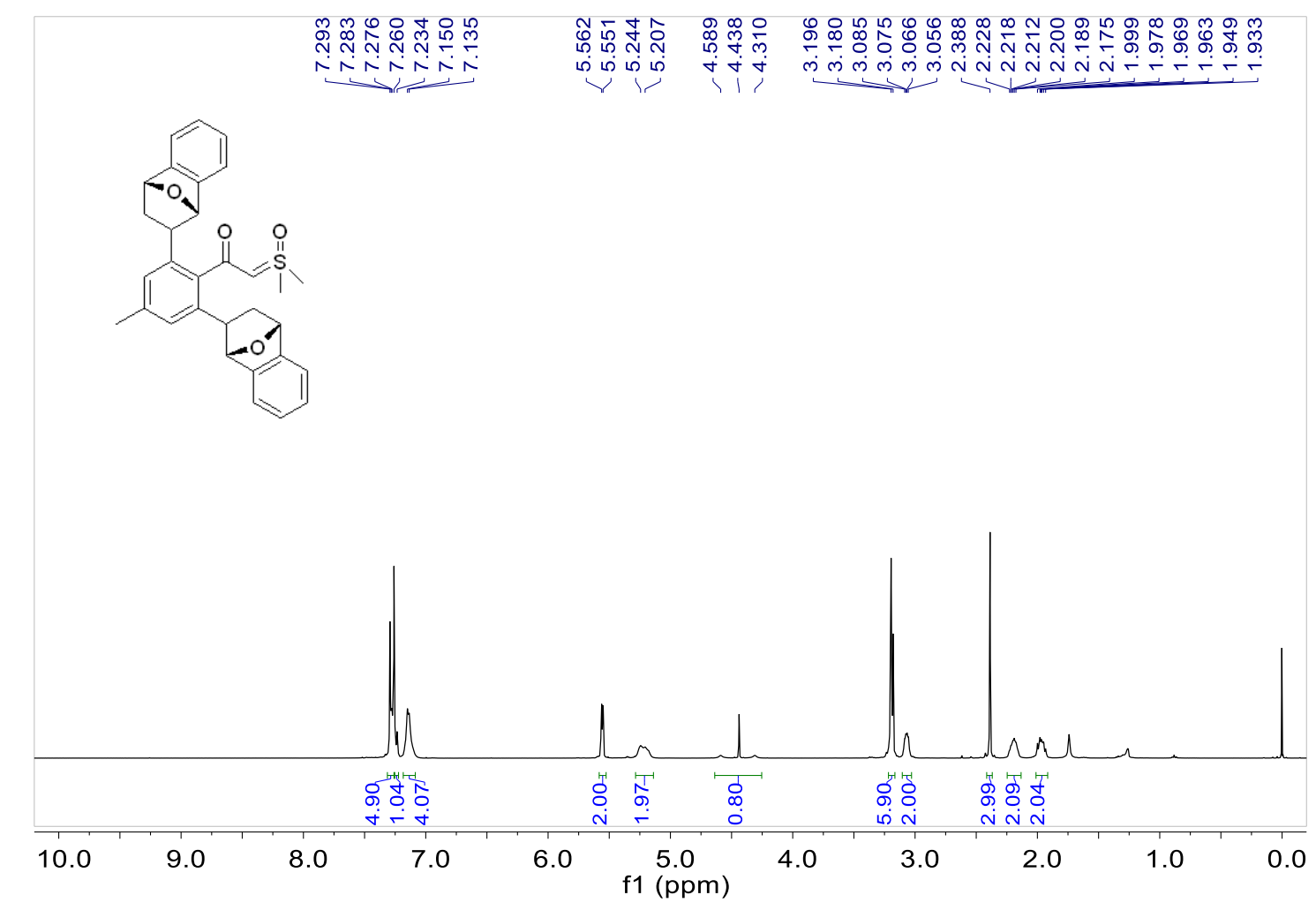

${ }^{1} \mathrm{H}$ NMR (400 MHz, $\mathrm{CDCl}_{3}$ ) spectrum of $6 \mathrm{ba}$

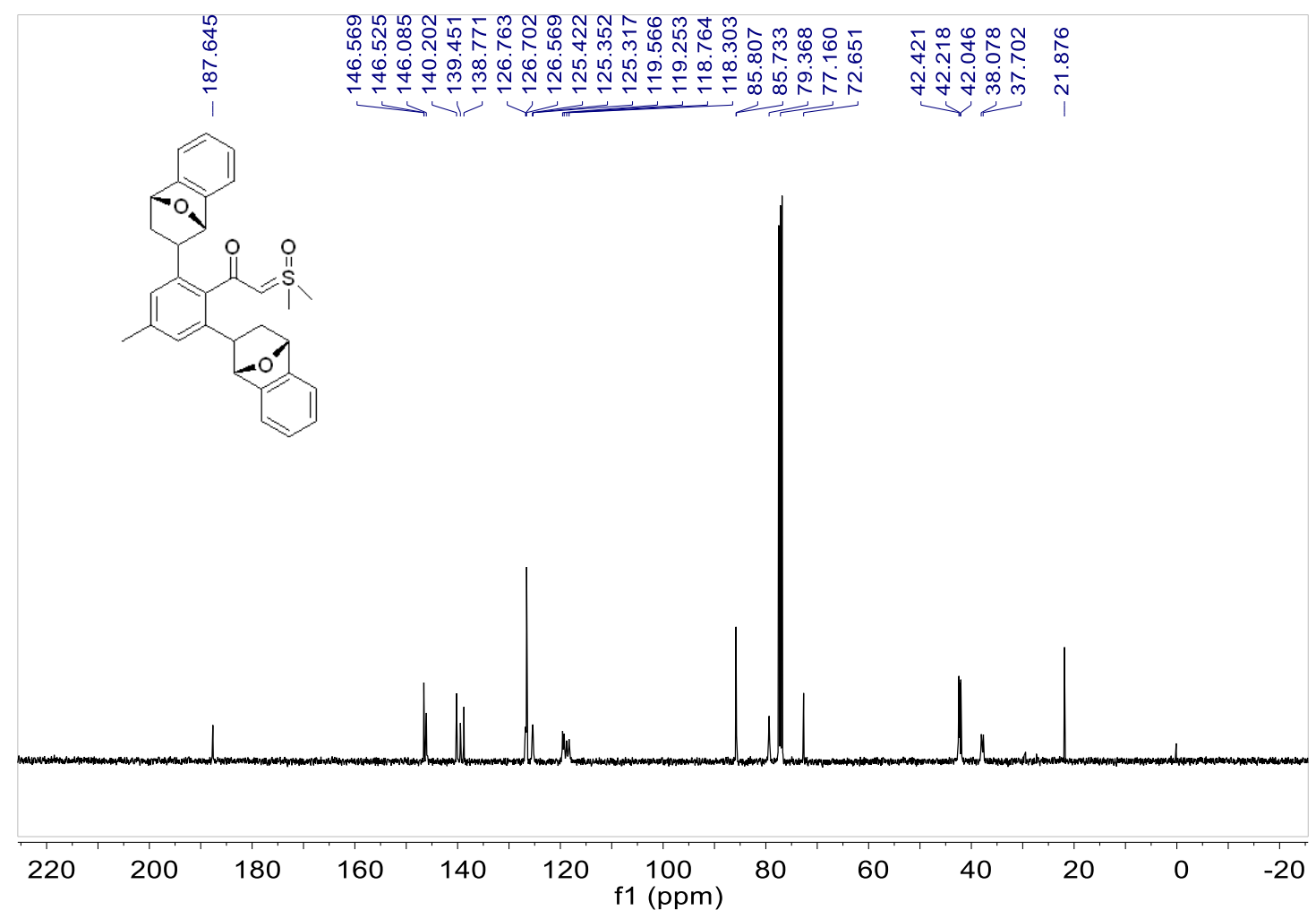

${ }^{13} \mathrm{C}$ NMR (100 MHz, $\left.\mathrm{CDCl}_{3}\right)$ spectrum of $6 \mathrm{ba}$ 


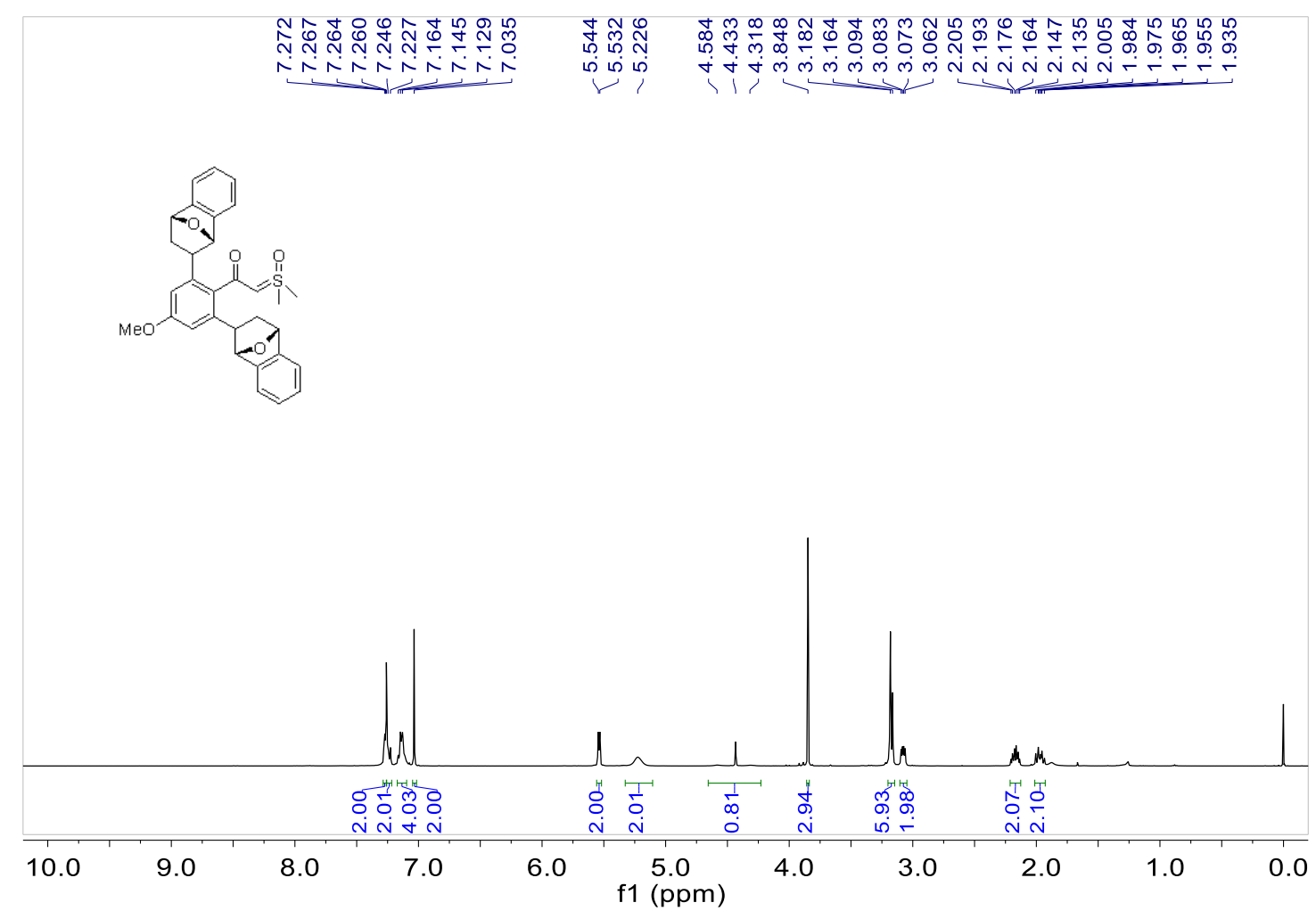

${ }^{1} \mathrm{H}$ NMR (400 MHz, $\mathrm{CDCl}_{3}$ ) spectrum of $6 \mathrm{ca}$

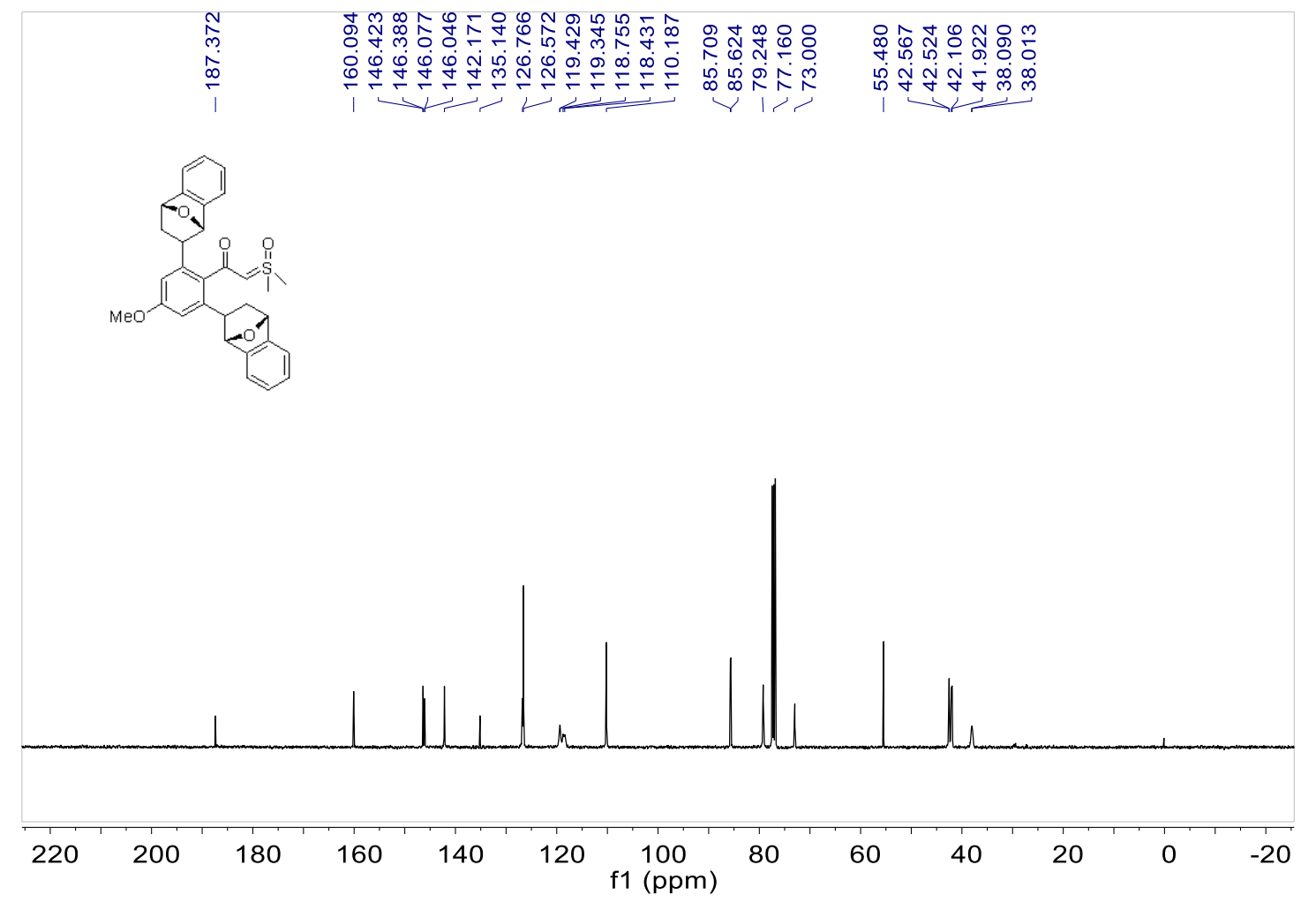

${ }^{13} \mathrm{C}$ NMR (100 MHz, $\mathrm{CDCl}_{3}$ ) spectrum of $6 \mathrm{ca}$ 


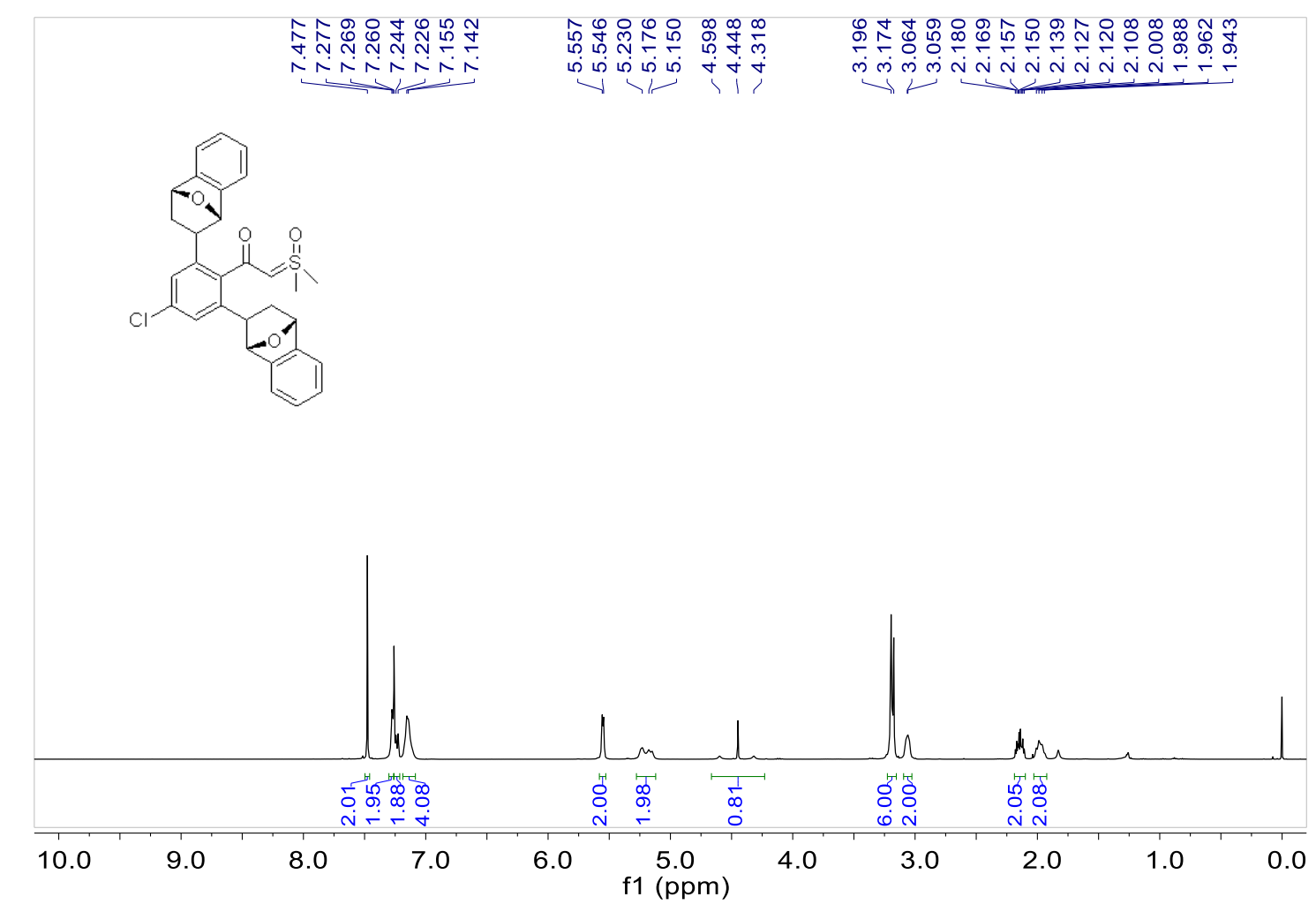

${ }^{1} \mathrm{H}$ NMR (400 MHz, $\mathrm{CDCl}_{3}$ ) spectrum of $6 \mathrm{da}$

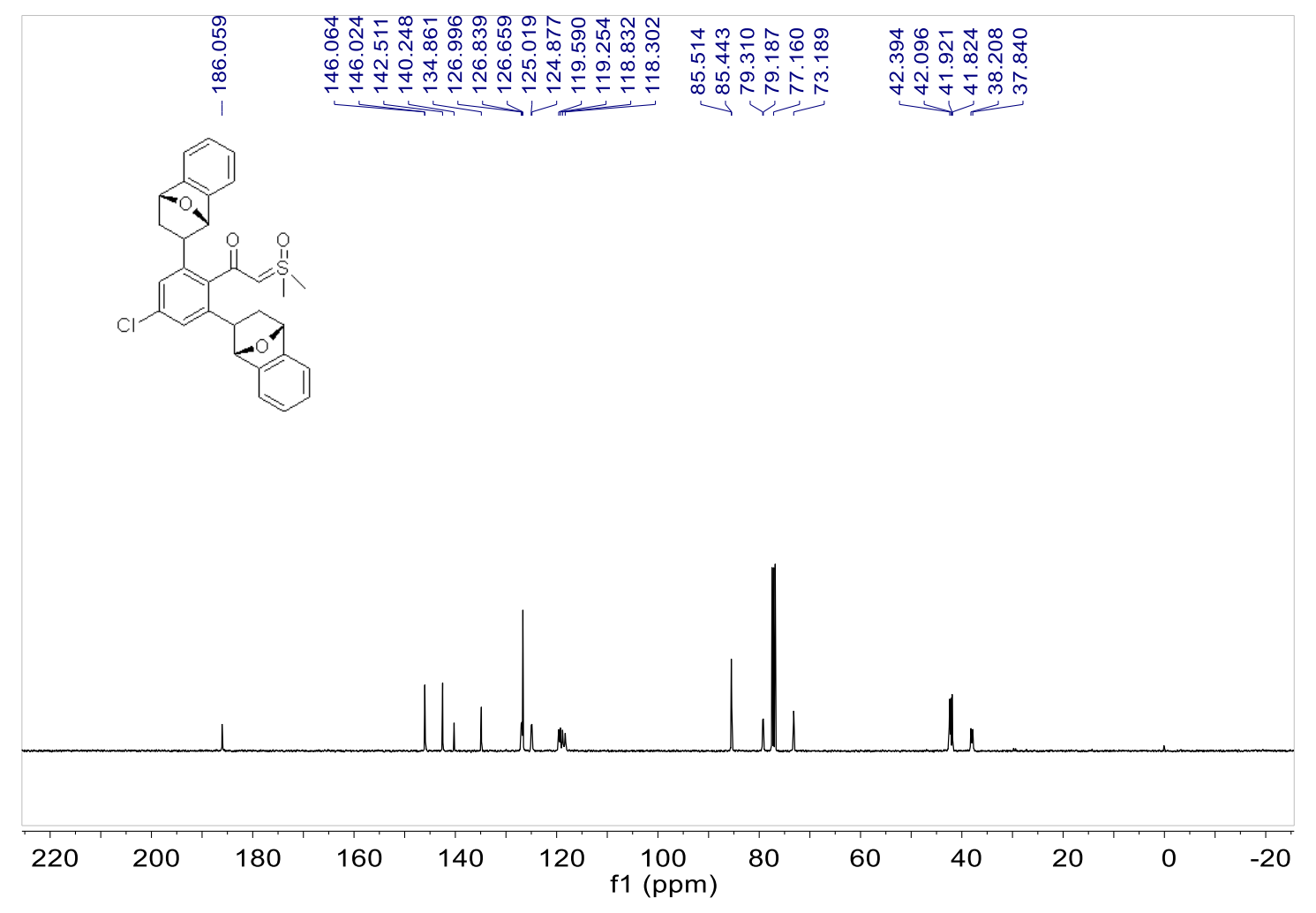

${ }^{13} \mathrm{C}$ NMR (100 MHz, $\mathrm{CDCl}_{3}$ ) spectrum of $6 \mathrm{da}$ 


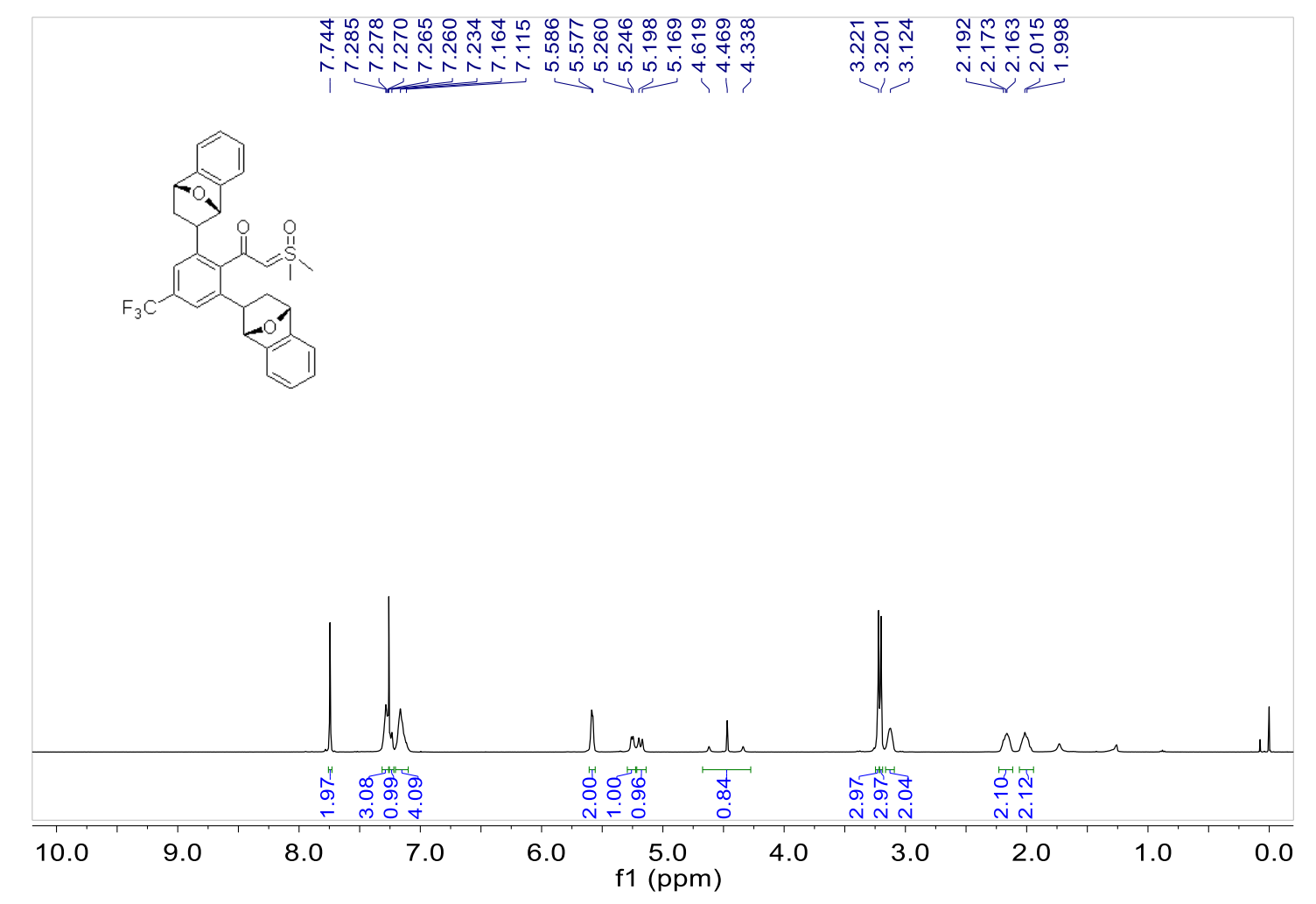

${ }^{1} \mathrm{H}$ NMR (400 MHz, $\mathrm{CDCl}_{3}$ ) spectrum of $6 \mathrm{ea}$

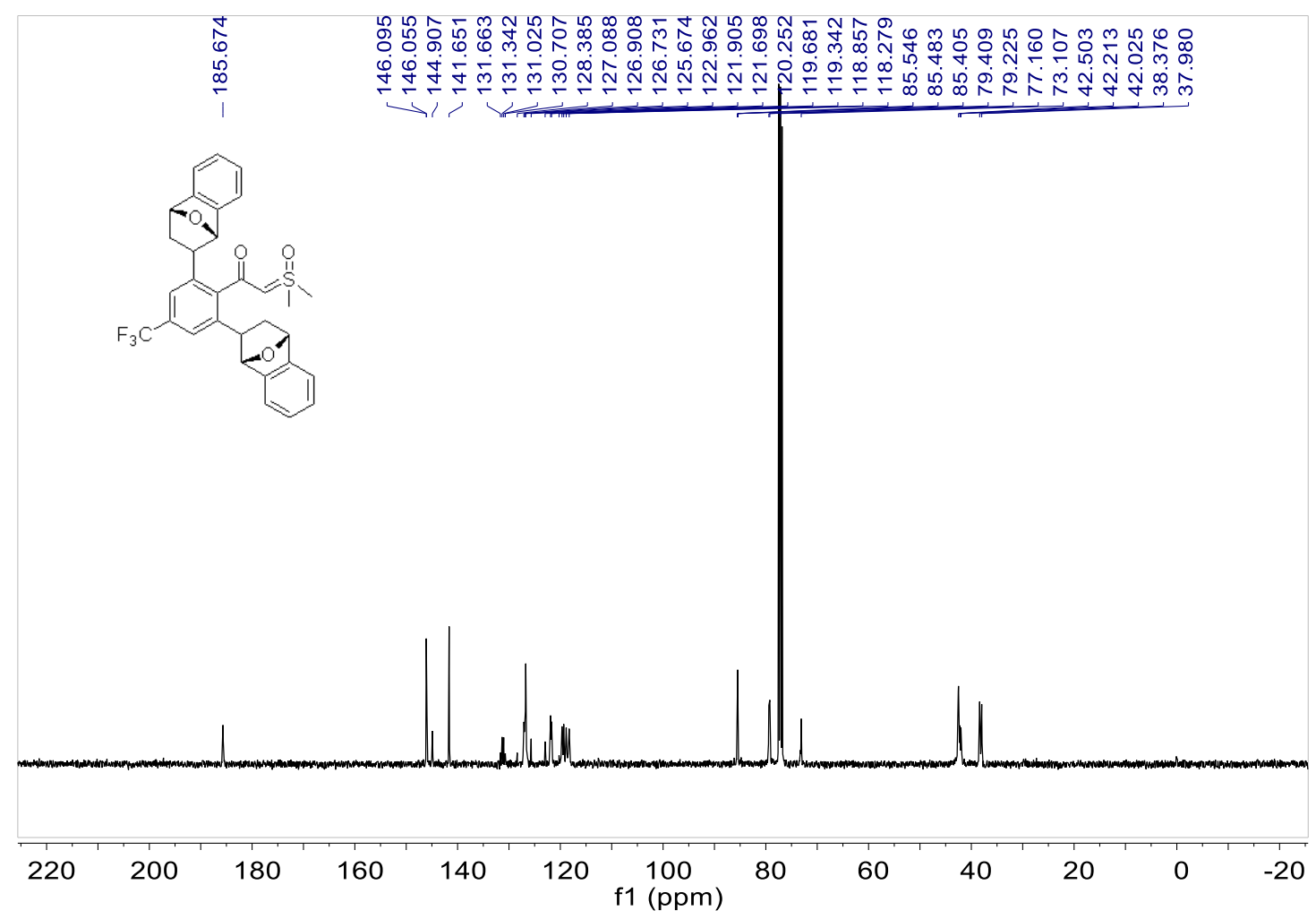

${ }^{13} \mathrm{C}$ NMR (100 MHz, $\mathrm{CDCl}_{3}$ ) spectrum of $6 \mathrm{ea}$ 


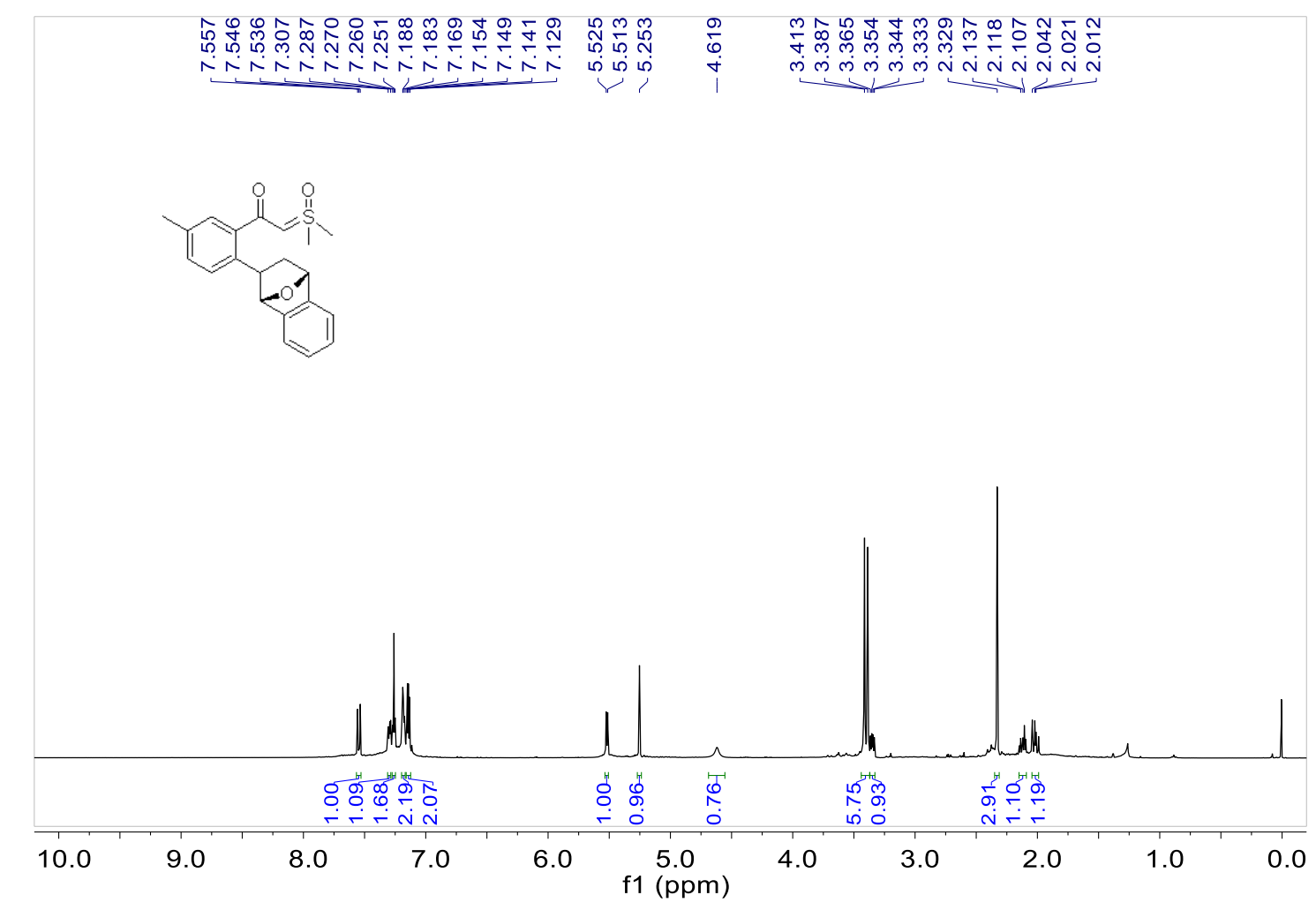

${ }^{1} \mathrm{H}$ NMR (400 MHz, $\mathrm{CDCl}_{3}$ ) spectrum of 6 fa

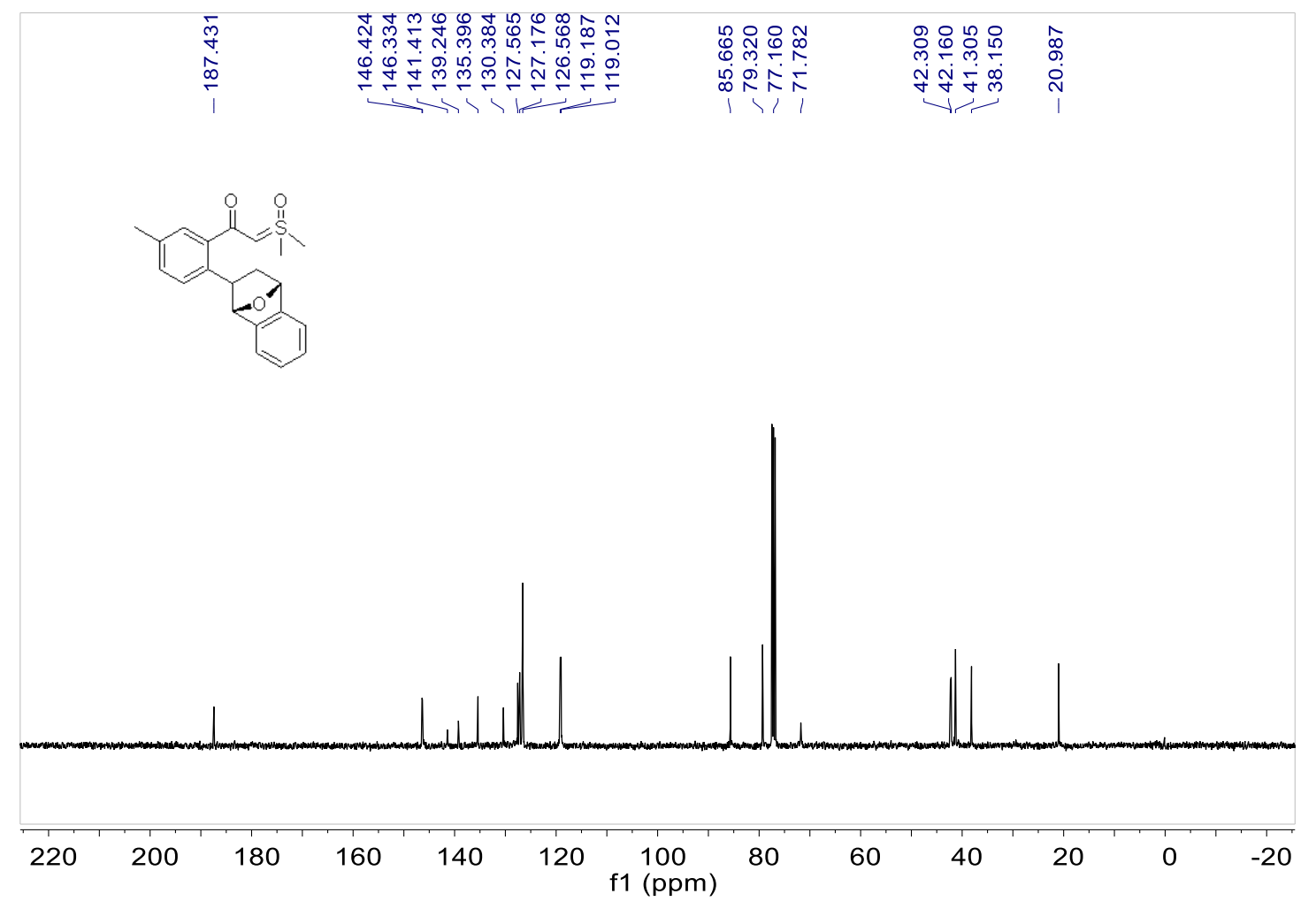

${ }^{13} \mathrm{C}$ NMR (100 MHz, $\mathrm{CDCl}_{3}$ ) spectrum of $6 \mathrm{fa}$ 

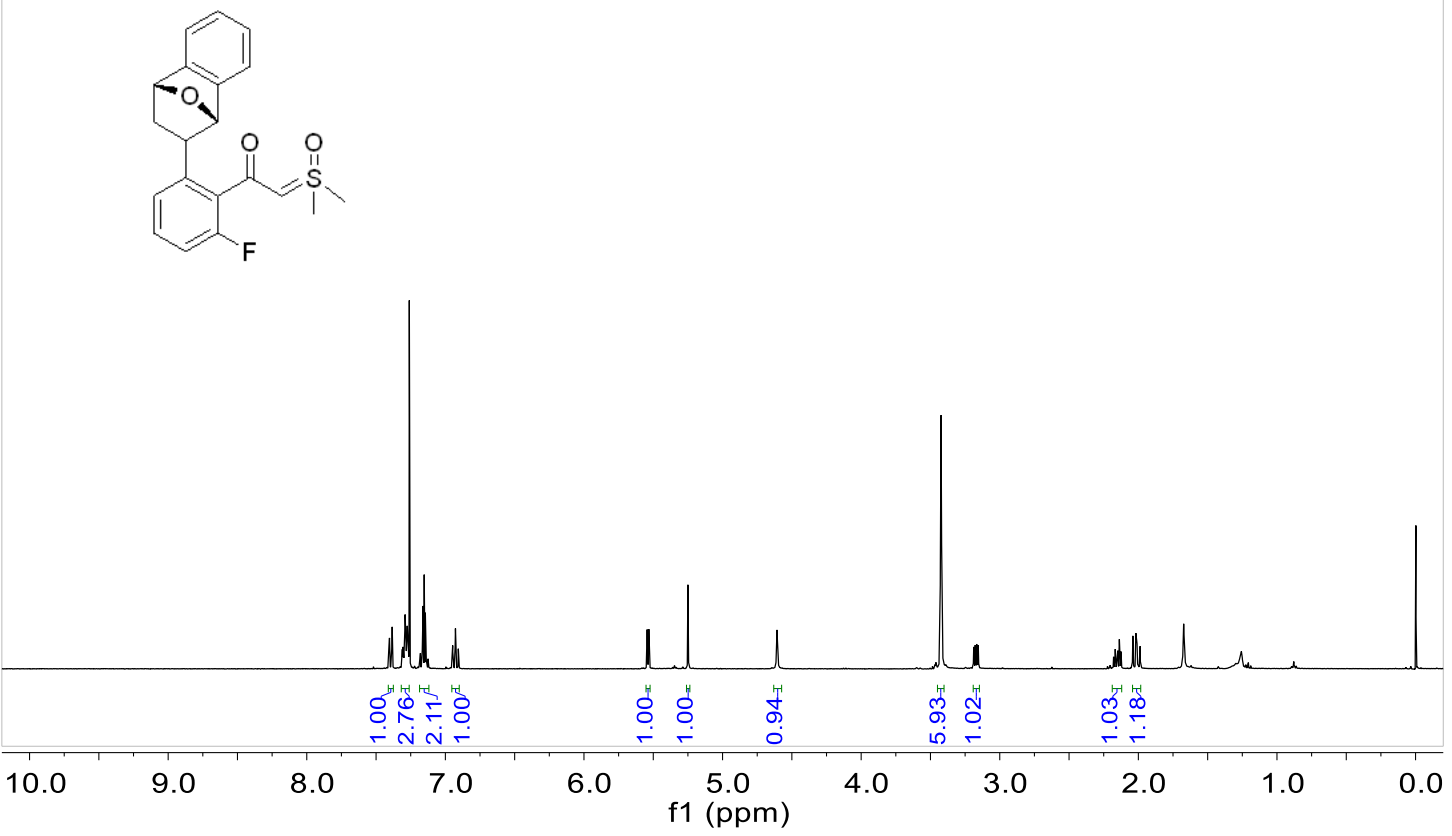

${ }^{1} \mathrm{H}$ NMR (400 MHz, $\mathrm{CDCl}_{3}$ ) spectrum of $6 \mathrm{ga}$

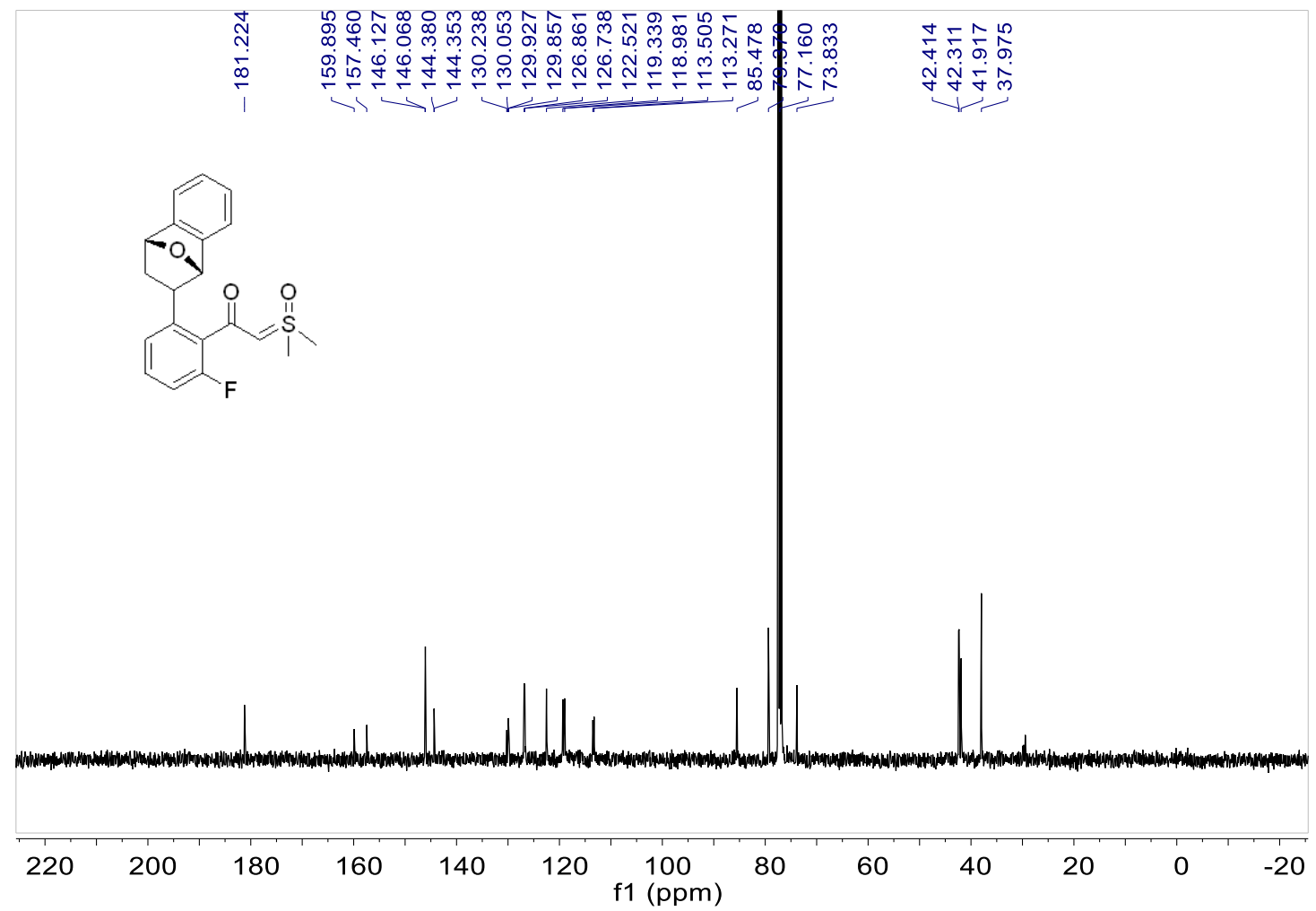

${ }^{13} \mathrm{C}$ NMR (100 MHz, $\left.\mathrm{CDCl}_{3}\right)$ spectrum of $6 \mathrm{ga}$ 


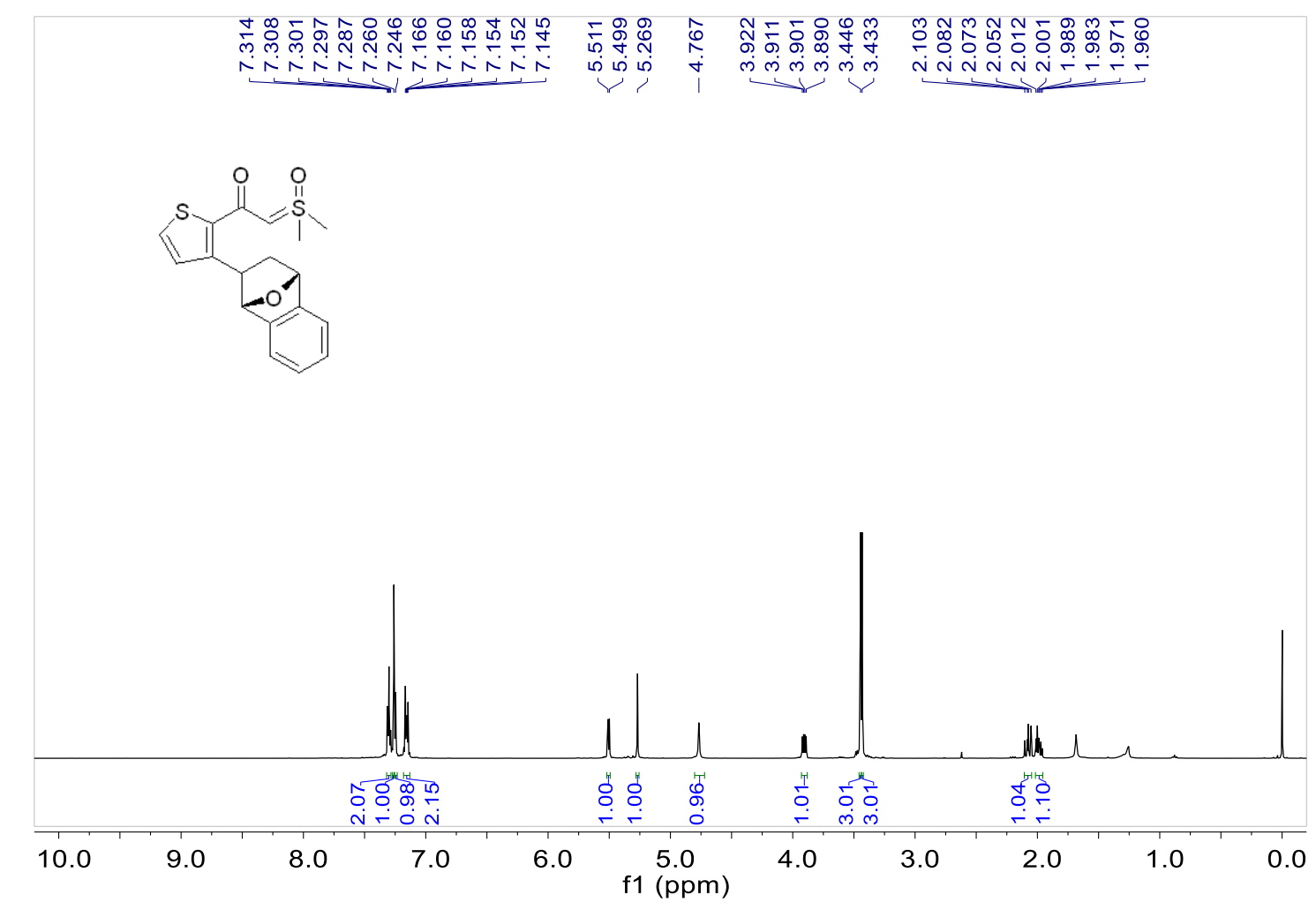

${ }^{1} \mathrm{H}$ NMR (400 MHz, $\left.\mathrm{CDCl}_{3}\right)$ spectrum of $6 \mathrm{ha}$

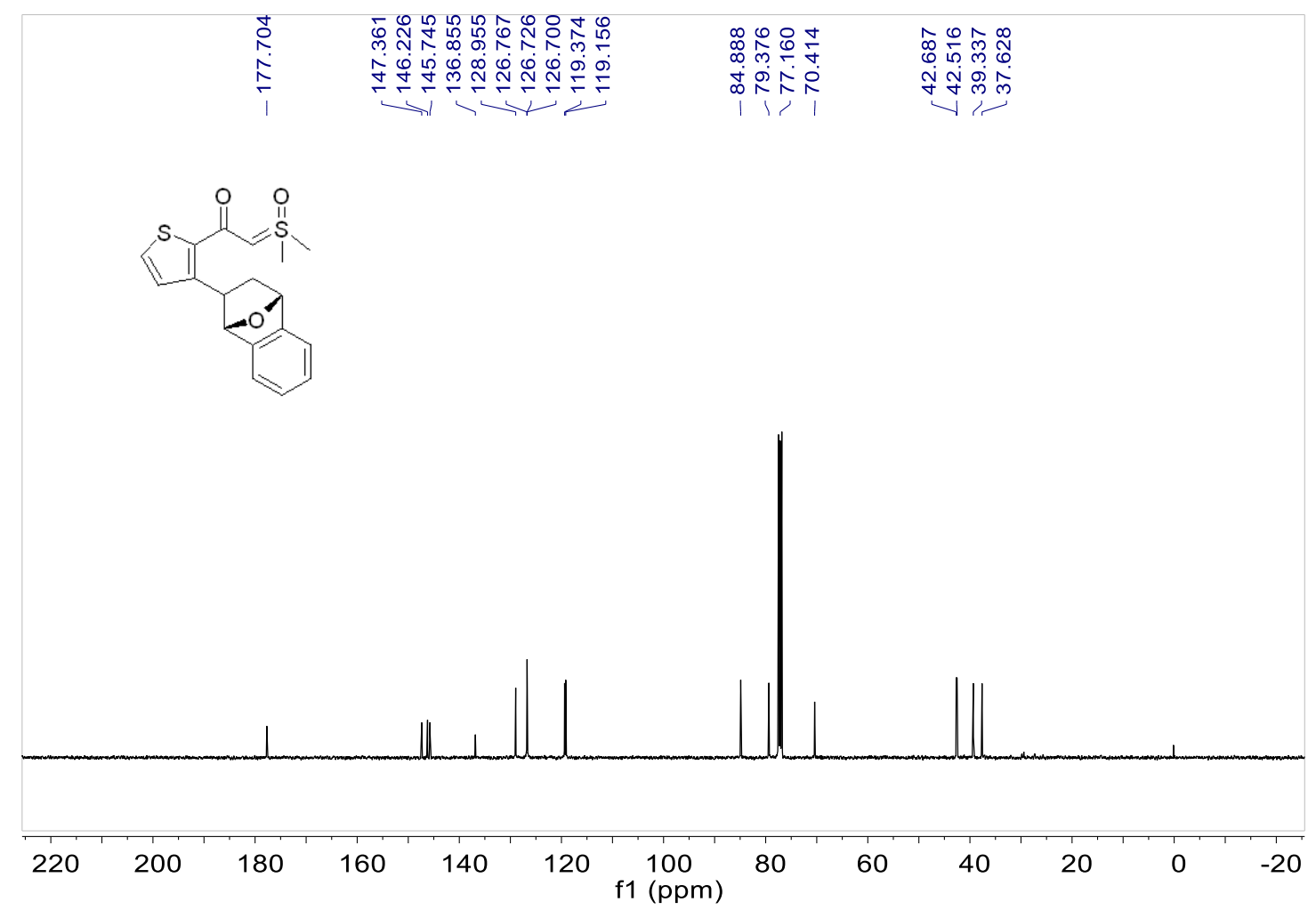

${ }^{13} \mathrm{C}$ NMR (100 MHz, $\mathrm{CDCl}_{3}$ ) spectrum of $6 \mathrm{ha}$ 


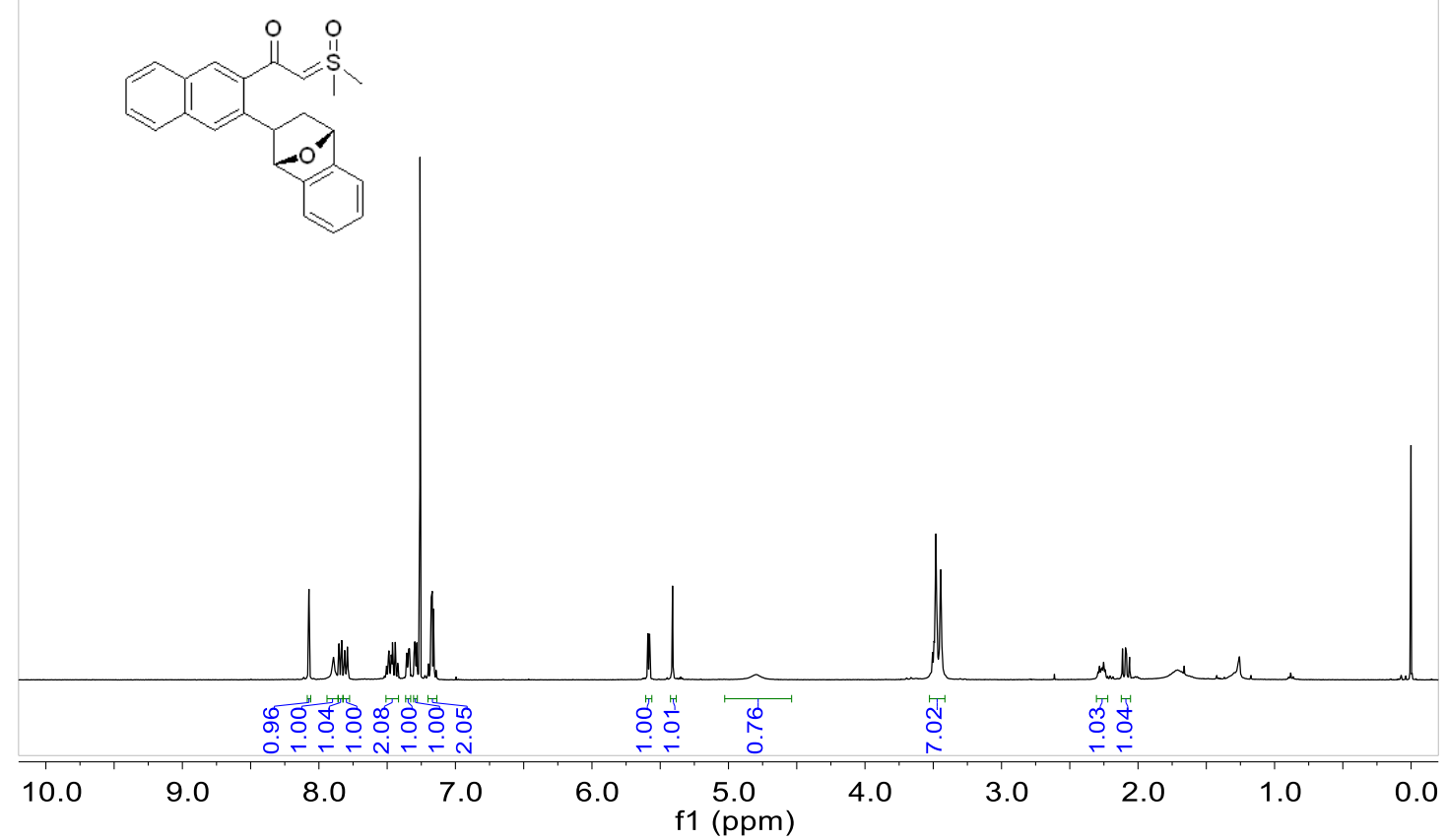

${ }^{1} \mathrm{H}$ NMR (400 MHz, $\mathrm{CDCl}_{3}$ ) spectrum of $6 \mathrm{ia}$

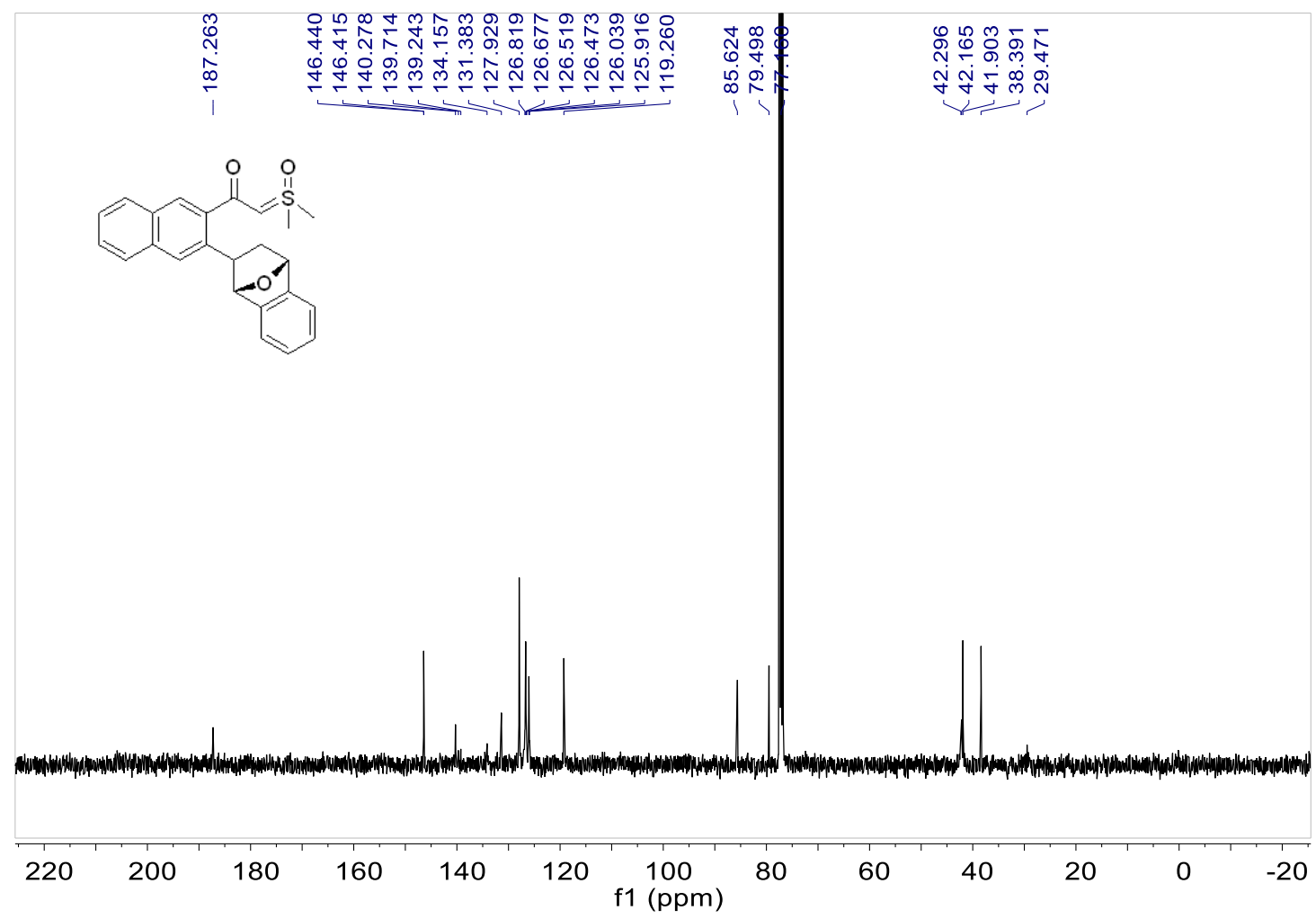

${ }^{13} \mathrm{C}$ NMR (100 MHz, $\left.\mathrm{CDCl}_{3}\right)$ spectrum of $6 \mathrm{ia}$ 


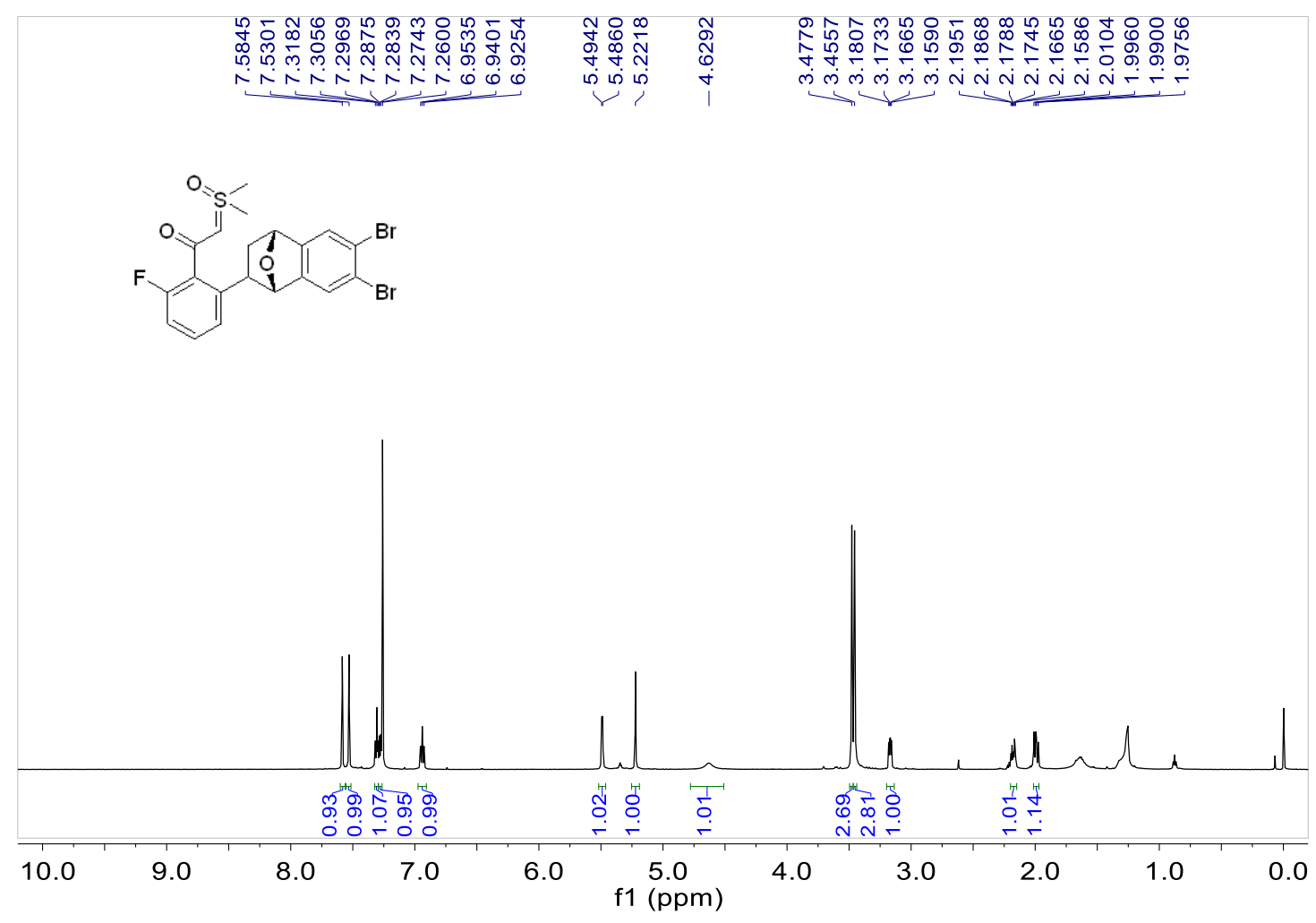

${ }^{1} \mathrm{H}$ NMR (600 MHz, $\left.\mathrm{CDCl}_{3}\right)$ spectrum of $6 \mathrm{gb}$

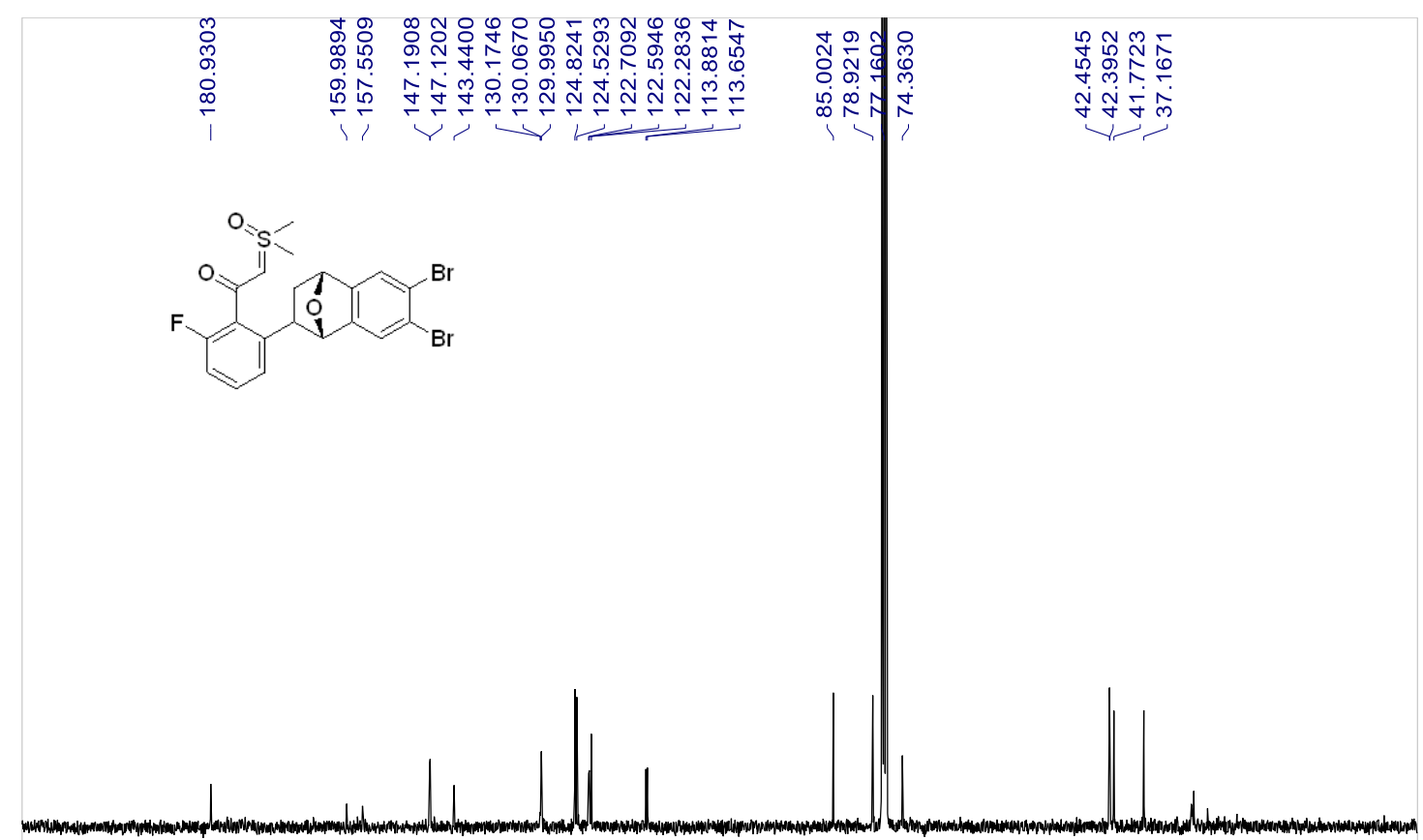

$10200190180170160150140130120110100 \quad 90 \quad 80 \quad 70 \quad 60 \quad 50 \quad 40 \quad 30 \quad 20 \quad 10 \quad 0$ f1 (ppm)

${ }^{13} \mathrm{C}$ NMR (100 MHz, $\left.\mathrm{CDCl}_{3}\right)$ spectrum of $6 \mathrm{gb}$ 

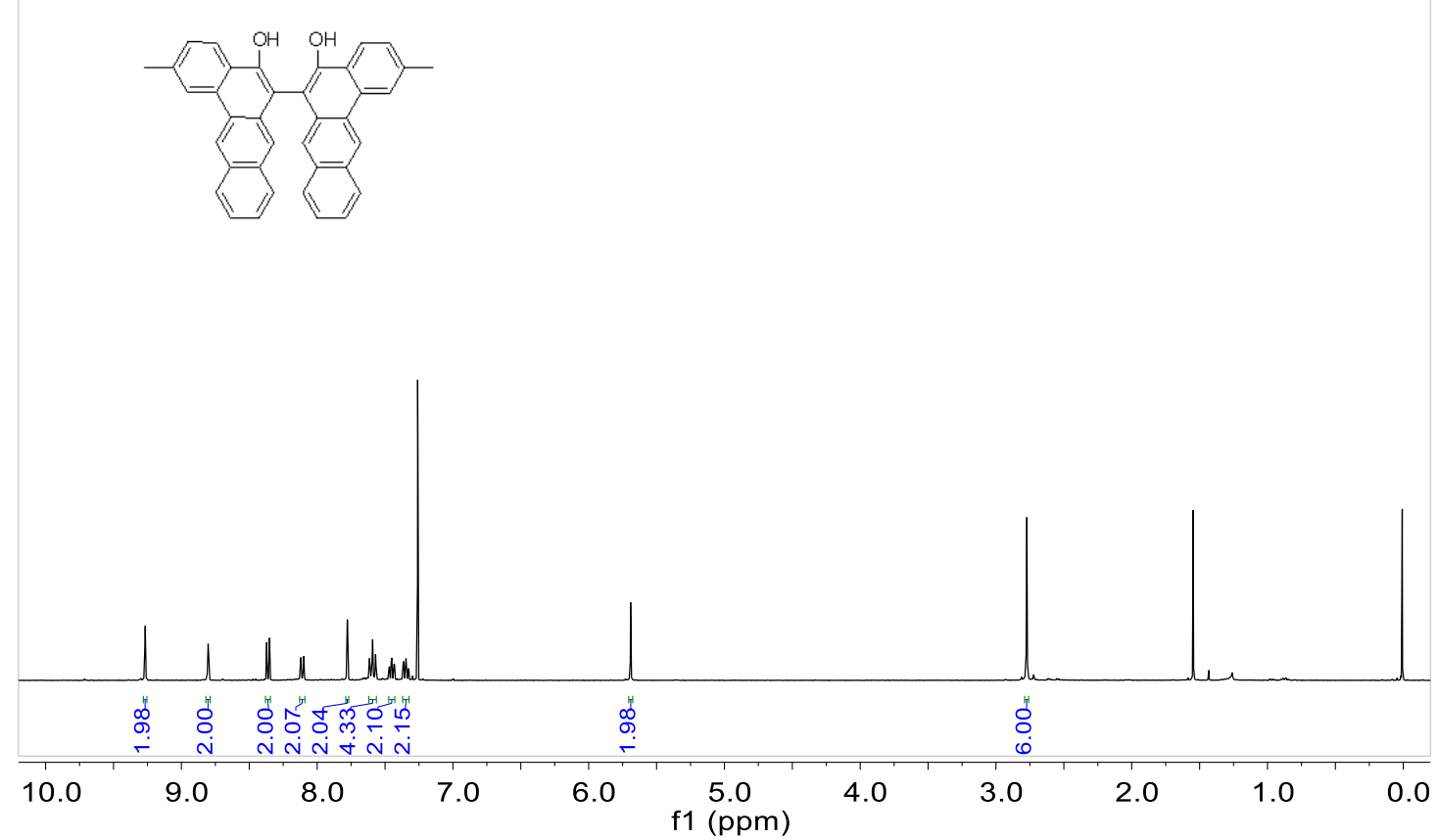

${ }^{1} \mathrm{H}$ NMR (400 MHz, $\mathrm{CDCl}_{3}$ ) spectrum of 7

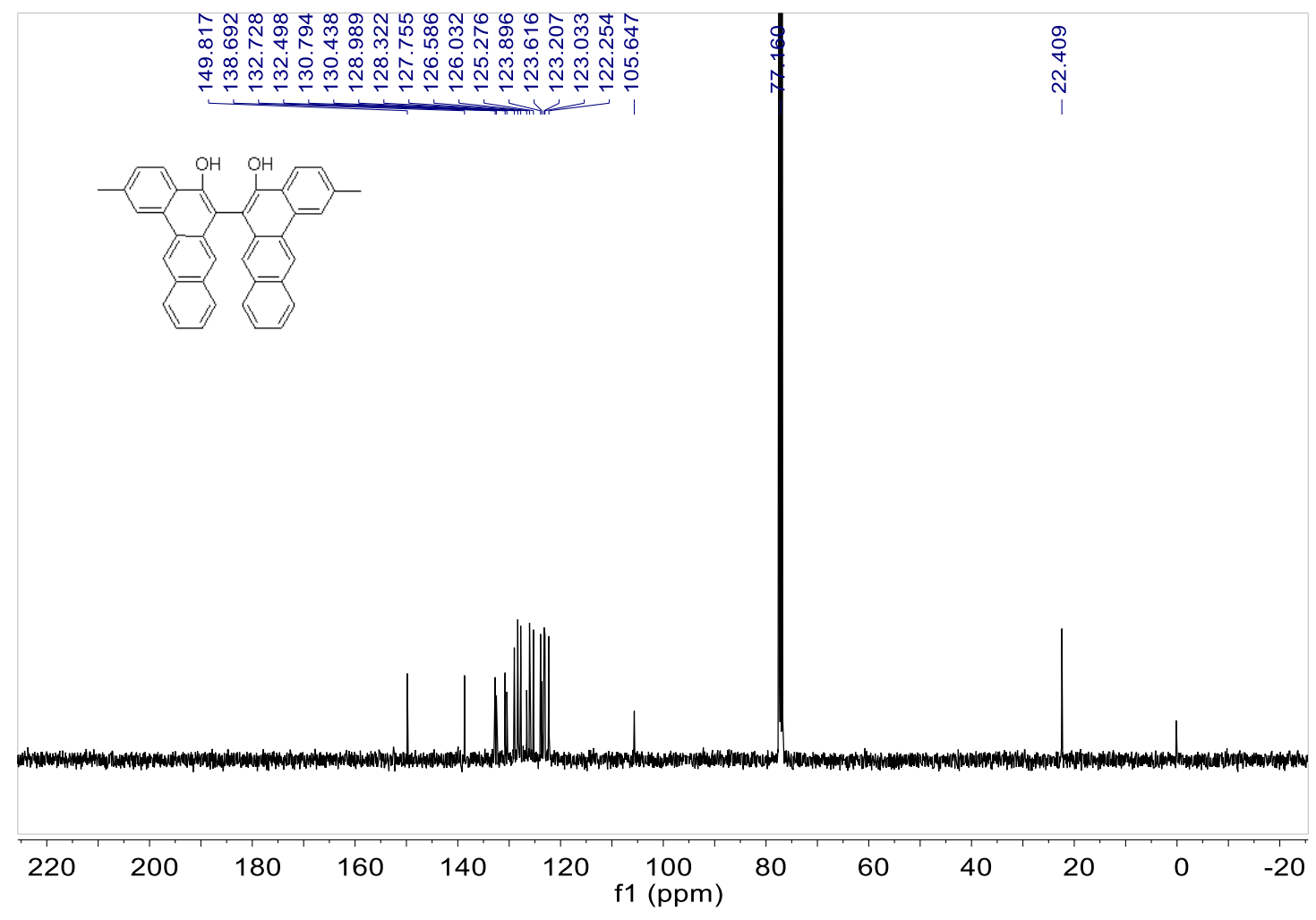

${ }^{13} \mathrm{C}$ NMR (100 MHz, $\left.\mathrm{CDCl}_{3}\right)$ spectrum of 7 

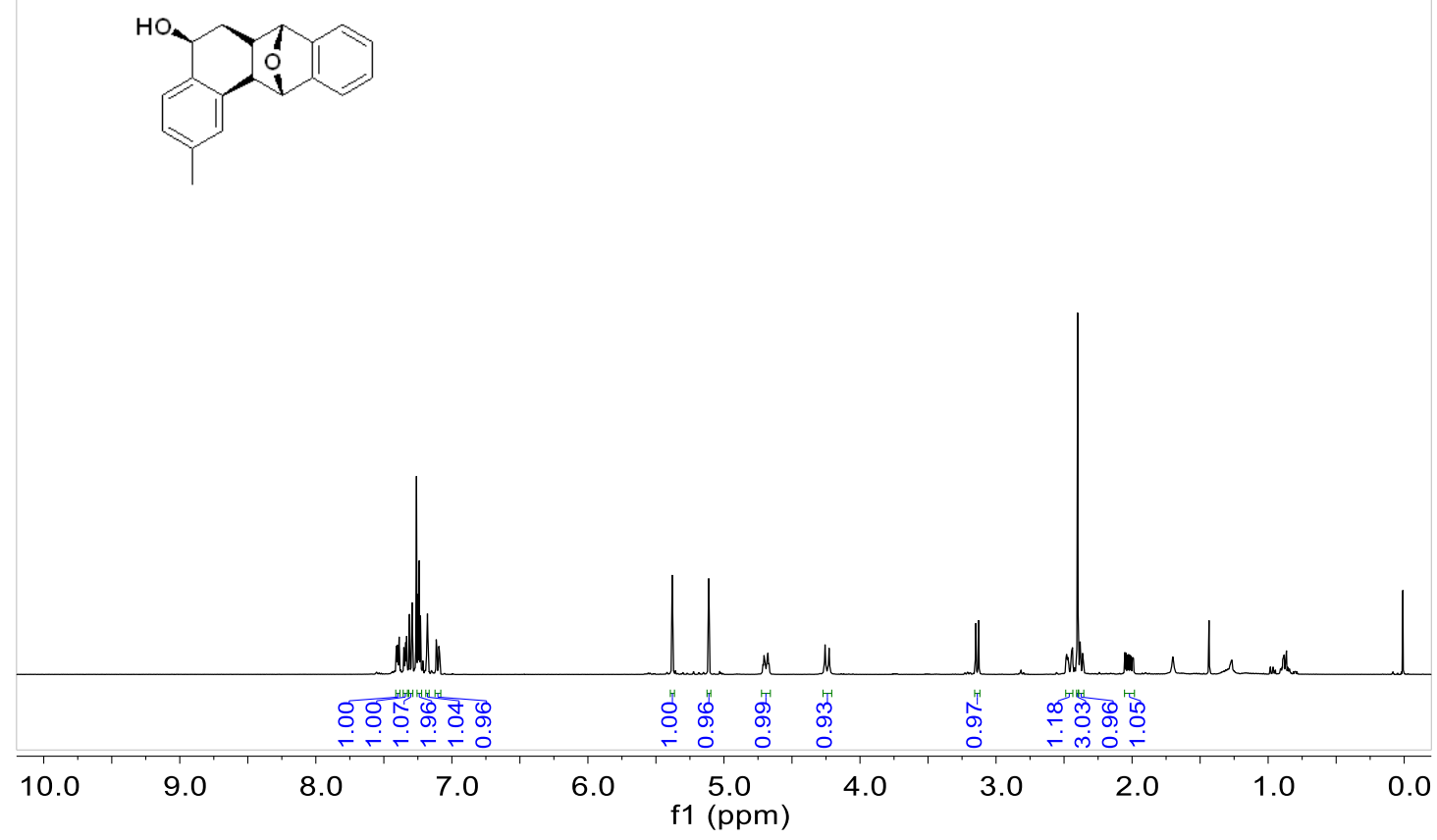

${ }^{1} \mathrm{H}$ NMR (400 MHz, CDCl$)$ spectrum of 8

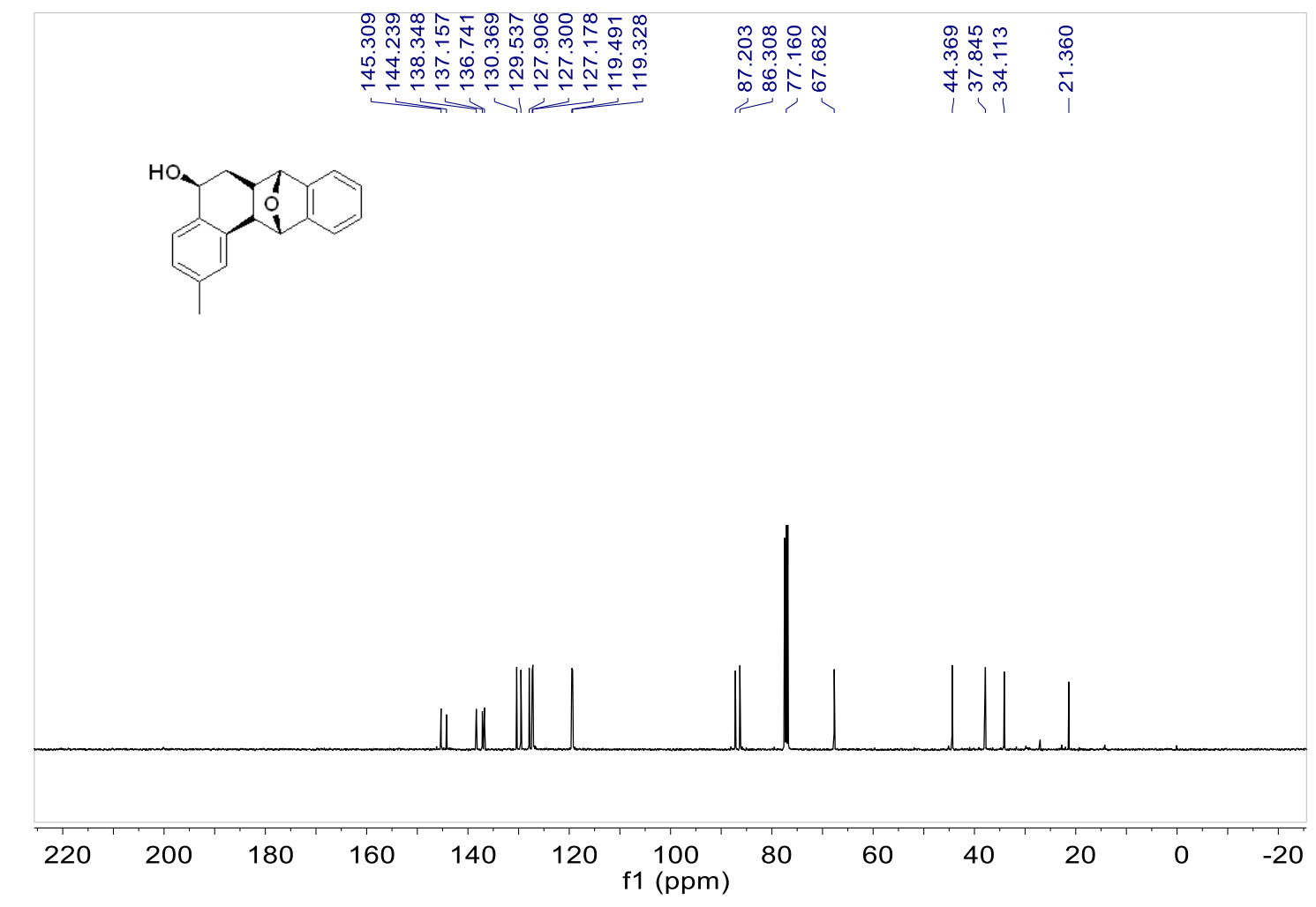

${ }^{13} \mathrm{C}$ NMR (100 MHz, $\left.\mathrm{CDCl}_{3}\right)$ spectrum of 8 